Prepared for the U.S. Department of Energy

under Contract DE-AC05-76RL01830

\title{
Filtration Understanding: FY10 Testing Results and Filtration Model Update
}

RC Daniel

JM Billing

CA Burns

RA Peterson
RL Russell

PP Schonewill

RW Shimskey

April 2011

Pacific Northwest

NATIONAL LABORATORY

Proudly Operated by Battelle Since 1965 



\title{
DISCLAIMER
}

This report was prepared as an account of work sponsored by an agency of the United States Government. Neither the United States Government nor any agency thereof, nor Battelle Memorial Institute, nor any of their employees, makes any warranty, express or implied, or assumes any legal liability or responsibility for the accuracy, completeness, or usefulness of any information, apparatus, product, or process disclosed, or represents that its use would not infringe privately owned rights. Reference herein to any specific commercial product, process, or service by trade name, trademark, manufacturer, or otherwise does not necessarily constitute or imply its endorsement, recommendation, or favoring by the United States Government or any agency thereof, or Battelle Memorial Institute. The views and opinions of authors expressed herein do not necessarily state or reflect those of the United States Government or any agency thereof.

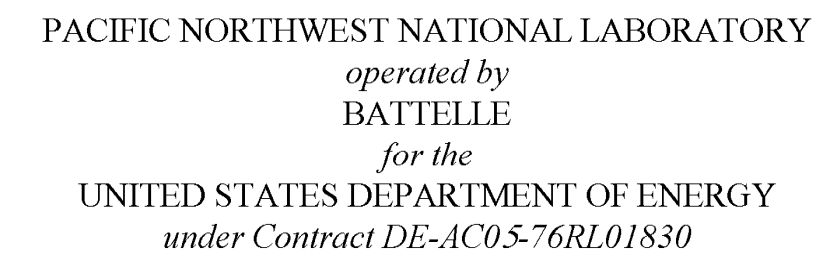

Printed in the United States of America

Available to DOE and DOE contractors from the Office of Scientific and Technical Information,

P.O. Box 62, Oak Ridge, TN 37831-0062;

ph: (865) 576-8401

fax: $(865)$ 576-5728

email: reports@adonis.osti.gov

\author{
Available to the public from the National Technical Information Service, \\ U.S. Department of Commerce, 5285 Port Royal Rd., Springfield, VA 22161 \\ ph: (800) 553-6847 \\ fax: $(703) 605-6900$ \\ email: orders@ntis.fedworld.gov \\ online ordering: http://www.ntis.gov/ordering.htm
}

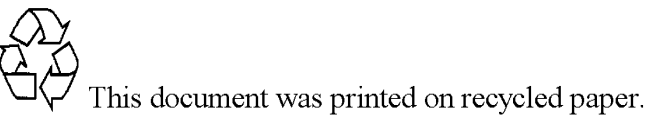

$(9 / 2003)$ 

PNNL-20299

EMSP-RPT-008

\title{
Filtration Understanding: FY10 Testing Results and Filtration Model Update
}

\author{
RC Daniel RL Russell \\ JM Billing PP Schonewill \\ CA Burns \\ RW Shimskey \\ RA Peterson
}

April 2011

Prepared for

the U.S. Department of Energy

under Contract DE-AC05-76RL01830

Pacific Northwest National Laboratory

Richland, Washington 99352 



\section{Summary}

This document completes the requirements of Milestone 2-4, Final Report of FY10 Testing, discussed in the scope of work outlined in the EM31 task plan WP-2.3.6-2010-1. The focus of task WP-2.3.6 is to improve the U.S. Department of Energy's (DOE's) understanding of filtration operations for high-level waste (HLW) to improve filtration and cleaning efficiencies, thereby increasing process throughput and reducing the $\mathrm{Na}$ demand (through acid neutralization). Developing cleaning/backpulsing requirements will produce much more efficient operations for both the Hanford Tank Waste Treatment and Immobilization Plant (WTP) and the Savannah River Site (SRS), thereby significantly increasing throughput by limiting cleaning cycles. Indeed, current program results derived from Pacific Northwest

National Laboratory (PNNL) studies indicate that a $35 \%$ increase in filter throughput can be achieved by optimization of backpulse frequency alone. In this report, the results of FY10 testing conducted under EM31 task plan WP-2.3.6-2010-1 are presented and discussed. The scope of this work is to develop the understanding of filter fouling to allow developing this cleaning/backpulsing strategy.

The overall approach to this task is:

- Review previous cross-flow testing at SRS, PNNL, and other peer review journals to direct testing activities for this task.

- Develop a predictive model that reflects the important physical mechanisms of fouling and cleaning for use in determining effective filter cleaning strategies for a variety of feeds.

- Develop simulants that can result in significant irreversible fouling, but also are representative of the types of materials that are present in HLW.

- Test these simulants in bench-scale equipment to identify the cleaning requirements for these feeds.

- Develop a cleaning strategy to optimize throughput while minimizing added Na.

- Validate the model and operational strategy with actual waste samples as appropriate.

The FY10 work discussed in the current report focused on tests that would improve understanding of filter fouling dynamics to allow development of improved filtration models. To this end, three filtration test programs were conducted to provide insight into dynamics of filter fouling and the efficacy of flux recovery operations, including both backpulsing of the filter and chemical cleaning regimens. The three testing programs were:

- Series 1, Long Term Fouling Tests - examined flux decline when filtering the Pretreatment Engineering Platform (PEP) waste simulant slurry for long periods of time without backpulsing. Previous filtration testing has only studied filter flux dynamics over relatively brief periods of operation (4-12 hours) with respect to expected filtration operation times at WTP (on the order of 100 hours). The aim of Series 1 testing was to answer this need.

- Series 2, Component Fouling Tests - determined the filtration behavior of the major components that comprise the PEP simulant. By examining each solid component separately, the nature of particle-particle and particle medium interactions can be evaluated by changing the suspending phase chemistry to gain insight about the filtration behavior under both normal operating conditions and changing chemical conditions (like those experienced during waste washing operations or filter cleaning). The goal of Series 2 testing was to determine the major fouling components in the PEP 
simulant waste slurry and to gain insight into role of particle-particle and particle-filter interactions in the overall flux behavior over a range of operating $\mathrm{pH}$.

- Series 3, Cleaning Tests and Filter Evaluation - determined the efficacy of filter flux restoration through chemical cleaning operations. Previous filtration testing has evidenced a decline in filter performance that cannot be recovered through backpulsing operations alone for both actual Hanford tank waste and waste simulant slurries. The goal of Series 3 testing was to provide additional testing of flux restoration by chemical cleaning regimens.

In addition to filtration testing, it was desirable to update the filtration model discussed in the literature report. The approach in this work is to build a model framework starting from the PNNL model reported in Daniel et al. (2010b). It was also anticipated that other theoretical approaches discussed in the survey of literature performed in Daniel et al. (2010a) would assist in building an understanding of cross-flow filtration behavior.

The major findings of Series 1 testing are as follows:

\section{- Continuous flux decay observed over 100-hour test periods.}

The filter flux continuously declined for all operating conditions and did not reach a steady-state filter flux. Extrapolation of the observed behavior indicates that the filter may block completely (i.e., exhibit zero flux) at finite time.

- Axial velocity increases correlated to increases to filter flux.

Use of high axial velocity improved filter flux relative to tests using lower axial velocity for the entire duration of test.

- Increases in transmembrane pressure correlated to increases in filter flux decay. Test results show that while high transmembrane pressure (TMP) yields higher initial filter fluxes, it also accelerates the rate of filter fouling significantly. On the other hand, operating at low transmembrane pressures yields lower initial filter fluxes, but the filter does not foul as strongly. The test results suggest that low transmembrane pressures may provide the most effective filter throughput if the filters are operated for long times without backpulsing or cleaning.

\section{- Backpulsing does not completely restore initial flux after cleaning.}

An approximately $25 \%$ flux loss occurred for all tests that could not be corrected by backpulsing. This poses significant uncertainty with respect to the long-time effectiveness of backpulsing. Specifically, there is concern that irreversible fouling could continue indefinitely (either as a result of repeated backpulsing or filtration) until flux can no longer be recovered through backpulsing.

The major findings of Series 2 testing are as follows:

\section{- Amorphous species in PEP simulant dominate filter fouling.}

Component baseline testing identified iron (III) oxy-hydroxide (FeOOH) and chrome (III) oxy-hydroxide $(\mathrm{CrOOH})$ solids as major fouling components that, even as 0.1 -vol\% suspensions, showed filter fluxes similar to $\sim 3$-vol\% PEP simulant slurries. In contrast, boehmite solids (also in 0.1 -vol\% suspensions) show filter fluxes that are one order of magnitude greater than typically observed for PEP simulant sludges. 
- Decreases in pH of solution chemistry below 7-8 change particle/filter interactions.

The filter flux of suspensions containing fine particles shows a strong dependence on solution $\mathrm{pH}$ (relative to that observed for suspensions of coarse particles). In addition, regions of "strong" fouling were observed for fine boehmite, $\mathrm{FeOOH}$, and $\mathrm{CrOOH}$ suspensions, indicating that charge effects may be important for cross-flow filtration of the PEP simulant.

The major findings of Series 3 testing are as follows:

- Improvement from chemical cleaning after PEP quantified.

Analysis of clean water flux (CWF) and manufacturer data indicates that the best clean water flux achievable for the Mott Grade 0.1 tubular filter elements used in cell unit filter (CUF) testing falls between 2-3 GPM/ $\mathrm{ft}^{2}$. After extended contact with PEP simulant slurry, filter cleaning with $0.5 \mathrm{M}$ oxalic acid produced initial values for CWF that ranged from 1.2 to $1.8 \mathrm{GPM} / \mathrm{ft}^{2}$.

- Difficulties in CWF measurements identified and discussed.

Assessing acid cleaning effectiveness has been complicated by a frequently observed rapid decline in CWF. It is suspected that trace solids not removed by acid cleaning cause this rapid decline. To overcome uncertainty in the measurement of CWF introduced by rapid decline, it is recommended that the initial CWF flux (i.e., that immediately following start-up of filter operations or immediately following a backpulse) be used to evaluate filter cleanliness. This recommendation avoids the need to accommodate flux decline from residual solids.

- Benchmark for “acceptable” CWF assessed.

Historically, CWF measurements exceeding $1 \mathrm{GPM} / \mathrm{ft}^{2}$ have been used to determine when the filter is "acceptably" clean. Analysis of CWF data and PEP simulant data indicates the deviation in initial flux for a PEP simulant slurry tested on a filter that achieved a CWF of $1 \mathrm{GPM} / \mathrm{ft}^{2}$ is less than $14 \%$ from the maximum achievable flux on a new filter.

\section{- Differences in nitric acid and oxalic acid test minimal.}

CWF testing was not able to associate poor historical performance of nitric acid in restoring filter flux to any major simulant component. For cleaning of FeOOH solids, which were identified as a major contributor to the PEP simulant fouling behavior, nitric acid cleaning was effective at restoring flux performance of a $\mathrm{SrCO}_{3}$ solution. Cleaning with oxalic acid did not appear to provide any additional benefit in terms of $\mathrm{SrCO}_{3}$ flux performance.

Examination of current PNNL models of long-time filtration showed the following:

- Existing filtration model unable to capture filtration behavior seen in Series 1 tests. The existing PNNL model was found to fit the first 12 hours of filtration behavior well. This was unsurprising, as the existing model was originally developed using 12 hours of flux data. The 12-hour fit was unsuccessful in predicting flux at longer times.

- Series 1 Testing demonstrated cake fouling behavior after approximately three hours. In lieu of using the existing PNNL model, a mechanistic approach was used to identify the mechanism(s) responsible for the long-term flux decay observed in the Series 1 tests. Each possible mechanism was tested first on a coarse time scale to identify regions of interest, and then an algorithm was used to solve for the optimum fit. By this technique, all Series 1 tests exhibited fouling that was due solely to cake formation and maturation after approximately 3 hours. 


\section{- Transition to intermediate fouling behavior after approximately 20 hours.}

By the same method used to identify the optimum cake mechanism, it was found that a transition to an intermediate fouling behavior occurred after approximately 20 hours of filtration. The intermediate fouling behavior is the sole contributor to the flux decay for the duration of the Series 1 tests. This indicates either that the cake has completely matured but the resistance continues to increase by the growth of sub-surface particle groups or it is a mathematical artifact due to the flux approaching an unknown, future (greater than 100 hours operating time) steady flux.

\section{- Filter flux predictions can be performed for times greater than 3 hours.}

Both the cake and intermediate mechanisms were fit to the filter flux data using one adjustable parameter. It was found that this adjustable parameter scales linearly with the ratio of the transmembrane pressure to the inertial pressure $(\Lambda)$. This relationship can be used to predict filter flux after 3 hours for tests at similar conditions with knowledge of only the dimensionless parameter $\Lambda$.

\section{- Appropriate model for initial filtration period difficult to identify.}

Efforts to use the mechanistic analysis at filtration times less than 3 hours were unsuccessful in identifying the controlling mechanism. Analysis in this period is complicated by sensitivity of the flux to inherent variability due to the statistical nature of turbulence, model dependence on the accurate resolution of initial flux, variability in operational start-up, and the extent of filter cleanliness. Some simple three parameter expressions were found to fit the initial data well but did not provide insight into the physical behavior.

While the FY10 testing program answered many questions about filtration performance, the following testing is planned in FY11 to resolve some of the outstanding issues identified in FY10 testing:

\section{- Planned 400-hour testing with 8-foot filtration system.}

The lack of observed filtration steady-state after 100 hours has significant implications on the throughput of filtration operations greater than 100 hours. Indeed, the current tests suggest that the filter will dead-end (go to zero flux) at finite time, necessitating flux recovery operations such as backpulsing or chemical cleaning. Extrapolation of filter flux results for one set of operating conditions indicates that the zero-flux time is $\sim 400$ hours. As such, PNNL recommends a 400 -hour filter test to verify the observed long-time flux behavior. To improve resolution, an 8-foot filtration element shall be installed to measure filter flux as low as $0.02 \mathrm{GPM} / \mathrm{ft}^{2}$.

\section{- Backpulse recovery testing.}

The long-time test results indicate a strong need for flux recovery operations. Backpulsing was shown to effectively restore a significant portion of the filter flux. However, a portion of the flux ( $25 \%$ ) could not be recovered by the single set of backpulses applied after 100 hours of continuous filtration. Irreversible flux loss with respect to backpulse is cause for concern, as the underlying mechanisms are not well understood. Additional testing is needed to confirm if irreversible fouling (filter conditioning) has a steady-state value or will continue degrade flux recovered upon backpulsing.

\section{- Expanded Series 1 Testing.}

The Series 1 testing showed how changes in slurry operations resulted in significant changes in throughput. In contrast to previous short-term tests, increases in transmembrane pressure were shown to demonstrate decreased filter performance without the aid of backpulsing. Testing also demonstrated that starting fluxes at different transmembrane pressures were not scalable to each 
other, demonstrating cake formation processes dependent on TMP. As such, the current tests illustrate the importance of understanding fouling dynamics with respect to the selected operating conditions as non-obvious process optimizations (such as lower transmembrane pressure) may result in better filter performance. Efforts to explore the impacts of temperature and solids concentrations are also planned as well.

\section{- Development of new simulants.}

The Series 2 testing demonstrated how different components behave differently with the stainless steel filter tested and that changes in waste composition likely will demonstrate different filtration behavior. To pursue the goal of this task, new simulants are planned to be developed based on characterization and filtration data of actual waste samples analyzed in the literature. Once filtration and chemical composition of the simulant correlate to actual waste data, testing performed on the PEP simulant this year will be duplicated to observe difference in long-term fouling behavior and the effectiveness of backpulsing and chemical cleaning. 



\section{Acronyms and Abbreviations}

$\begin{array}{ll}\text { AV } & \text { axial velocity } \\ \text { CUF } & \text { cell unit filter } \\ \text { CWF } & \text { clean water flux } \\ \text { DACS } & \text { data-acquisition collection system } \\ \text { DI } & \text { deionized } \\ \text { DOE } & \text { U.S. Department of Energy } \\ \text { EFRT } & \text { External Flowsheet Review Team } \\ \text { EMSL } & \text { William R. Wiley Environmental Molecular Sciences Laboratory } \\ \text { ft } & \text { square feet } \\ \text { GPM } & \text { gallons per minute } \\ \text { HDI } & \text { "How Do I,” the standards-based management system for PNNL } \\ \text { HLW } & \text { high-level tank waste } \\ \text { Hz } & \text { hertz } \\ \text { PEP } & \text { Pretreatment Engineering Platform } \\ \text { PNNL } & \text { Pacific Northwest National Laboratory } \\ \text { psig } & \text { pounds per square inch gauge } \\ \text { psid } & \text { pounds per square inch differential } \\ \text { PSD } & \text { particle size distribution } \\ \text { PTF } & \text { Pretreatment Facility } \\ \text { PUREX } & \text { plutonium-uranium extraction } \\ \text { REDOX } & \text { reduction oxidation } \\ \text { SEM } & \text { scanning electron microscopy } \\ \text { SRNL } & \text { Savannah River National Laboratory } \\ \text { SRS } & \text { Savannah River Site } \\ \text { TMP } & \text { undissolved solids } \\ \text { UDS } & \text { Hanford Tank Waste Treatment and Immobilization Plant } \\ \text { WTP } & \end{array}$





\section{Contents}

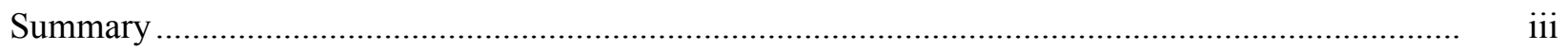

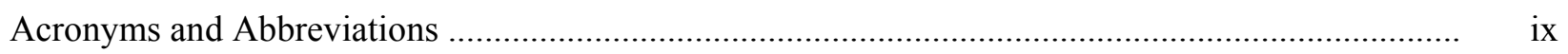

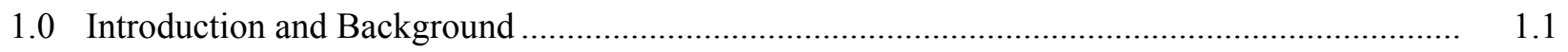

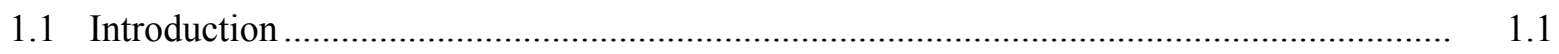

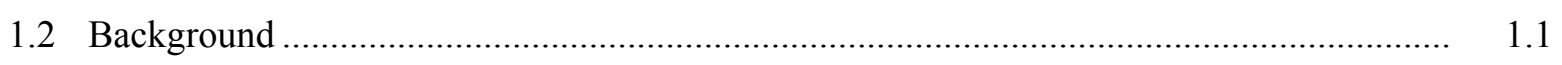

1.3 Need for Additional Fouling and Chemical Cleaning Studies ......................................... 1.3

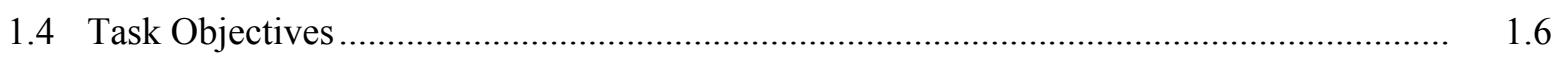

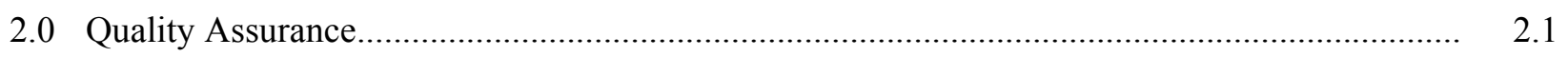

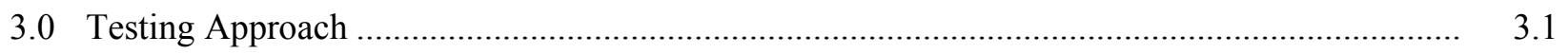

3.1 Series 1 Testing: Long-Term Fouling Tests .............................................................. 3.2

3.2 Series 2 Testing: Component Fouling Tests............................................................... 3.3

3.3 Series 3 Testing: Cleaning Tests and Filter Evaluation ............................................... 3.5

3.3.1 Cleaning Tests Associated with Series 1 ....................................................... 3.6

3.3.2 Cleaning Tests Associated with Series 2 ....................................................... 3.8

3.3.3 Strontium Carbonation Cleaning Tests ............................................................ 3.8

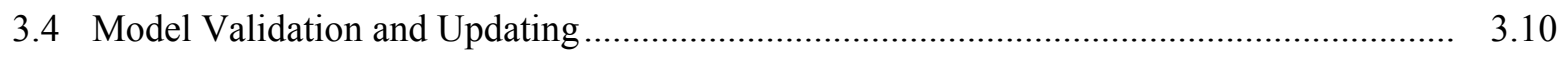

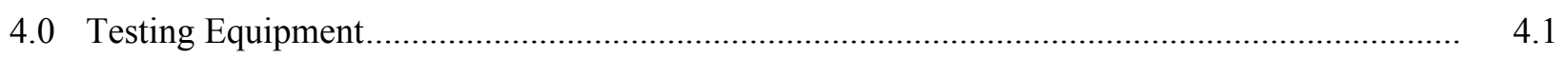

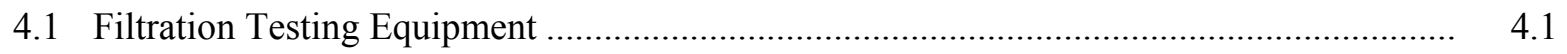

4.1.1 Cross-flow Filter ..................................................................................... 4.1

4.1.2 Cross-flow Filtration Testing System.............................................................. 4.4

4.1.3 Instrumentation and Data-Acquisition System................................................. 4.7

4.1.4 Operations of Cell Unit Filter and Sampling....................................................... 4.9

4.2 Simulant Characterization Equipment...................................................................... 4.10

4.2.1 Solid Concentration Analysis ........................................................................... 4.10

4.2.2 Slurry and Supernate Rheological Measurements.............................................. 4.10

4.2.3 Slurry and Supernate Density Measurements.................................................... 4.11

4.2.4 Particle Size Analysis............................................................................... 4.11

4.2.5 Isoelectric Point (IEP) Measurements .................................................................. 4.12

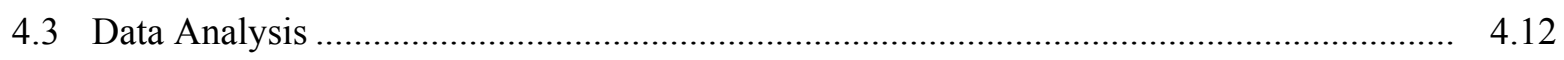

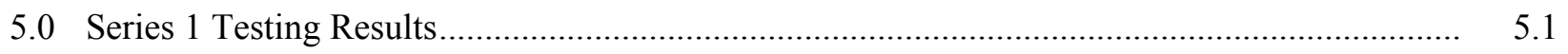

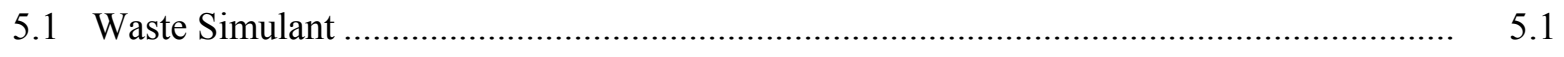

5.2 Test Results and Observations ............................................................................. 5.4

5.2.1 Flux Behavior Before Backpulsing ................................................................ 5.6

5.2.2 Flux Behavior After Backpulsing .................................................................. 5.9

5.2.3 Backpulsing Effectiveness ...................................................................... 5.12

5.3 Series 1 Test Conclusions ............................................................................. 5.13 


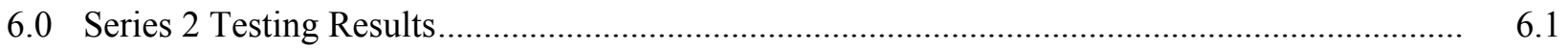

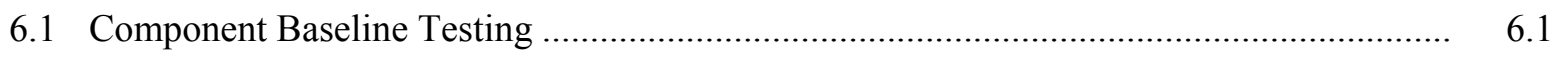

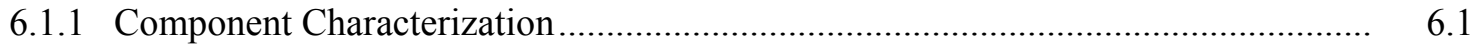

6.1.2 Component Test Results................................................................................ 6.4

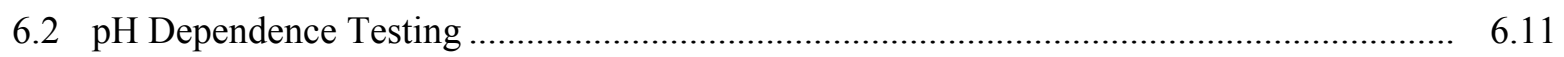

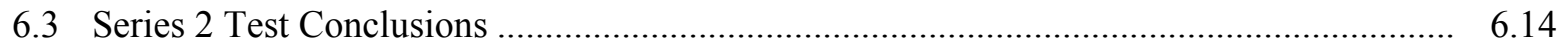

7.0 Series 3 Testing Results.............................................................................................. $\quad 7.1$

7.1 Estimates of Clean Filter Flux ............................................................................... 7.1

7.2 Series 1 Cleaning Test Results ................................................................................. 7.3

7.3 Series 2 Cleaning Test Results ............................................................................... 7.8

$7.4 \mathrm{SrCO}_{3}$ Cleaning Test Results ............................................................................. 7.9

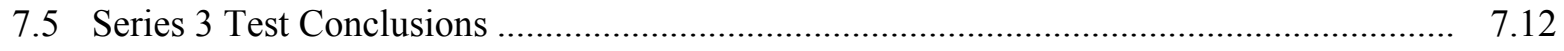

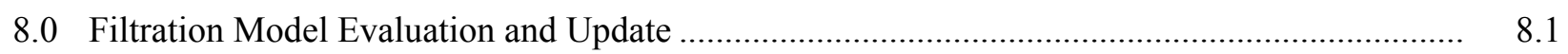

8.1 Comparison of Data to Existing Filter Flux Model........................................................... 8.1

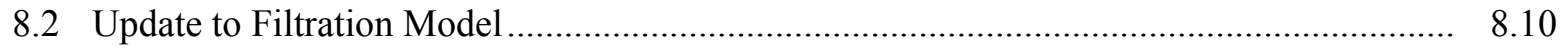

8.3 Implication to Operations Strategy ……………………............................................ 8.22

8.4 Limitations to Model and Required Testing Needed ………………………………..... 8.23

9.0 Summary and Recommendations ..................................................................................... 9.1

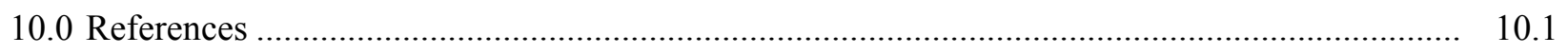

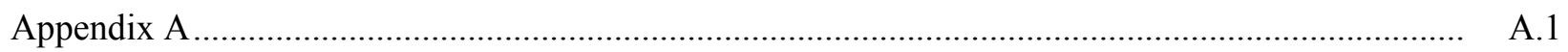

\section{Figures}

1.1 Dead-End and Cross-flow Filtration Configurations ……….................................................. 1.2

3.1 Overview of Cleaning Steps and CWF Tests During Series 1 Testing....................................... 3.7

4.1 Cell Unit Filter Element.................................................................................................... 4.2

4.2 SEM Image of CUF Filter Element Cross-Section ………................................................... 4.2

4.3 Cell Unit Filter Assembly Sketch ................................................................................... 4.3

4.4 The Cell Unit Filter Assembly …………………….................................................... 4.3

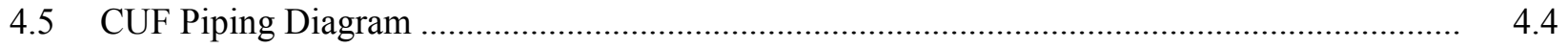

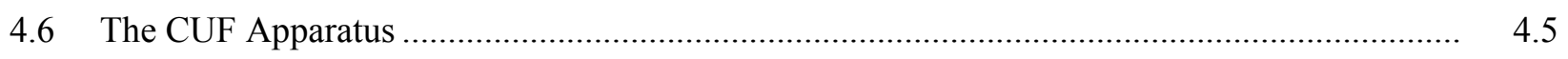

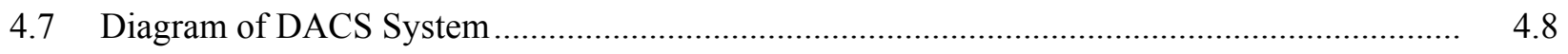

4.8 Digital Images of DACS Display Windows ....................................................................... 4.9

4.9 CUF 1-Minute Averaged Data Before and After Filtering Using Dixon's Q Test ..................... 4.13

5.1 Average Particle Size Distribution of PEP Simulant Before and After 100-hour Filter Fouling Tests ……………………………………………………………. 5.2

5.2 Comparison of Filter Flux Decline Profiles for PEP Simulant from the Current EM31 Study and CUF Low-Solids Test \#1 
5.3 Typical Series 1 Filtration Test Result with Time Scaled Linearly and

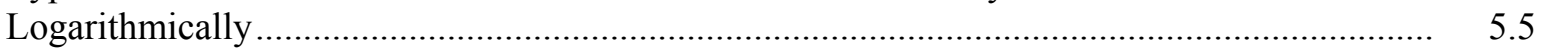

5.4 Corrected Permeate Flux Decline Over Initial 100-Hours at Different Axial Velocities ........... $\quad 5.7$

5.5 Corrected Permeate Flux Decline Over Initial 100-Hours at Different Transmembrane Pressures

5.6 Corrected Permeate Flux Decline Following Backpulse at 100-Hours of Filtration Time

5.7 Comparison of Flux Behavior Before and After Backpulsing

5.8 Comparison of Initial Flux at Start of Test and Flux Immediately Following Backpulsing at 100-Hours.

6.1 Differential and Cumulative Particle Size Distribution of Materials Used in Component Testing.

6.2 Results of Component Baseline Testing for Coarse and Fine Boehmite Solids

6.3 Comparison of Component Baseline Testing Results for Coarse Boehmite and Iron Oxy-hydroxide $(\mathrm{FeOOH})$ Solids....

6.4 Comparison of Component Baseline Testing Results for Coarse Boehmite and Chrome Oxy-hydroxide $(\mathrm{CrOOH})$ Solids

6.5 Comparison of FeOOH Filter Flux Behavior at Different Background Concentrations of $\mathrm{NaNO}_{3}$......

6.6 Comparison of Filter Flux for Coarse and Fine Boehmite Solids during pH Dependence Testing......

6.7 Comparison of Filter Flux for Coarse Boehmite and FeOOH Solids during pH Dependence Testing.

6.8 Comparison of Filter Flux for Coarse Boehmite and CrOOH Solids during $\mathrm{pH}$ Dependence Testing....

6.9 Comparison of Filter Flux for FeOOH Solids at Different Background Concentrations of $\mathrm{NaNO}_{3}$ during $\mathrm{pH}$ Dependence Testing.

6.10 Summary of Component Testing Fluxes Measured During the Initial and Final 12-Hour Period of Continuous Filtration...

7.1 Clean Water Flux for Series 1 Tests 1-5

7.2 Calculated Percentage Contribution of $\mathrm{R}_{\text {res }}$ to $\mathrm{R}_{\text {tot }}$ as a Function of Clean Water Flux for Series 1 Testing Based on the Assumptions in Table 7.4

7.3 Calculated $R_{\text {res }}$ to $R_{\text {tot }}$ Values for Series 1 Testing Based on the Assumptions in Table 7.4

7.4 Clean Water Flux Before and After Each Series 2 Test.

7.5 Flux During $\mathrm{SrCO}_{3}$ Slurry Testing Following Series 2, Test 2

7.6 Comparison of Flux During the Three $\mathrm{SrCO}_{3}$ Tests in the Cleaning Sequence...

7.7 Clean Water Flux Measurements Made During $\mathrm{SrCO}_{3}$ Cleaning Tests ...

7.8 Expected Impact of Fouling Not Removed by Cleaning on the Initial Filter Flux for a PEP Simulant Slurry That Has a Best Initial Flux of $0.10 \mathrm{GPM} / \mathrm{ft}^{2}$.

8.1 Original Model Fit to S1T1 Data before Backpulsing ......................................................... 8.4

8.2 Cake Equilibrium Constant vs. Dimensionless Pressure Ratio $(\Lambda)$ for All Series 1 Tests.......... 8.5 
8.3 Original Model Fit to the First 12 Hours of Filter Data from S1T1 .......................................

8.4 Dimensionless Membrane and Cake Resistance as a Function of Time for the Model Fit to S1T1 Data....

8.5 Dimensionless Membrane and Cake Resistance as a Function of Time for the Modified Model Fit to S1T1 Data ...

8.6 Modified Model Fit to S1T1 Data Before Backpulsing Using Constraint in Equation 8.9 ........

8.7 Modified Model Fit to S1T1 Data Before Backpulsing and After Backpulsing Using Constraint in Equation 8.9

8.8 Cake Equilibrium Constant as Estimated Using the Constraint in Equation 8.9 and Using the Backpulse Data with the Same Constraint vs. Dimensionless Pressure Ratio $\Lambda$ for all Series 1 Tests

8.9 Linearized Blocking Laws for S1T1 at Long-Times: $\mathrm{f}(\mathrm{J})$ Versus V, with Linear Regression Applied for Data from $t>10, t>25$, and $t>50$ Hours.

8.10 The Root-Mean Squared Error for the Regression Performed Using the Four Linearized Mechanistic Models on S1T1 Flux Data for Long-Times

8.11 Linearized Blocking Laws for S1T1 at Intermediate Times: $\mathrm{f}(J)$ Versus $V$, with Linear Regression Applied for Data from $5<t<10,5<t<15$, and $5<t<20$ Hours...

8.12 The Root-Mean Squared Error for the Regression Performed Using the Four Linearized Mechanistic Models on S1T1 Flux Data for Intermediate Times..

8.13 Optimum Fit to S1T1 Flux-Time Data Using the Model of Equation 8.23..... 8.18

8.14 Long-Time Optimum Fit for S1T1 Data Using the Intermediate Mechanism Model . 8.19

8.15 Optimum Fit to S1T1 Flux-Time Data Using the Model of Equation 8.25.... 8.20

8.16 Intermediate Time Optimum Fit for S1T1 Data Using the Cake Mechanism Model.

8.17 Fitting Parameters Obtained for the Optimal Intermediate Mechanism and the Cake Mechanisms vs. the Ratio of the Transmembrane Pressure to the Inertial Pressure......

\section{Tables}

1.1 Partial Listing of Reports with Filtration Testing that Supported Resolution of Issues Involving Filtration at WTP.....

1.2 Task Objective Milestones

3.1 AV/TMP Matrix for Series 1 Testing.

3.2 Common Parameters Used for Series 2 Filtration Testing

3.3 Series 2 Test Matrix for the Five Baseline Tests

3.4 Test Outline for $\mathrm{SrCO}_{3}$ Cleaning Tests..........................................................................

4.1 Summary of Malvern Mastersizer 2000 Instrument Information ............................................ 4.11

4.2 CUF Filtration System Measurement Instrumentation ........................................................... 4.12

5.1 Physical Properties of PEP Simulant Slurry Measured During Series 1 Testing........................ 5.2

5.2 Particle Size Distribution Table for PEP Simulant Before and After 100-Hour Filter Fouling Tests. 
5.3 Comparison of Series 1 Mean Values for Physical Properties to Range of PEP Test

Values

5.4 Comparison of Initial Flux and Flux After Backpulsing

5.5 Filter Throughput Results During First 100 Hours of Series 1 Testing...

6.1 Tabular Particle Size Distribution of Materials Used in Component Testing ....

6.2 Estimate of Potential Volume of Particle that Can Infiltrate and Foul CUF Filter Element Pores

6.3 Isoelectric Points of Series 2 Test Components.....

7.1 Isopropanol Flux Data from Rubow and Jha (1999) for a 316-L Stainless Steel Filter with a Thickness of 0.055 Inches.

7.2 Results of Regression Analysis of Flux Data and Calculation of Baseline CUF Filtration Rates for Clean Water and PEP Simulant Supernate

7.3 Comparison of Initial Permeate Flux with Pre-test CWF for Each of the Series 1 Tests

7.4 Assumptions for Calculating the Percentage Contribution of $\mathrm{R}_{\mathrm{res}}$ to $\mathrm{R}_{\mathrm{tot}}$ as a Function of Clean Water Flux for Series 1 Tests

8.1 Results of the Non-Linear Regression Applied to the Series 1 Data.

8.2 Results of the Non-Linear Least Squares Minimization Applied to the Series 1 Data Using the Criterion of Equation 8.9

8.3 Results Obtained from Optimum Intermediate Mechanism Model of Series 1 FluxTime Data

8.4 Results Obtained from Optimum Cake Mechanism Model of Series 1 Flux-Time Data 



\subsection{Introduction and Background}

\subsection{Introduction}

Current baseline technologies are in place or under development to treat high-level waste (HLW) prior to stabilization. The objective of pretreating the waste prior to immobilization is to partition the highly important radionuclides into a high-level fraction that comprises a small volume and partitioning the non-radioactive waste constituents into a low-level, large volume fraction. Several key waste constituents have strong influences over the amount of low-activity and high-activity waste volumes. These influences may control their solubility in the immobilized leading to increase volume. Conversely, sodium is added to the waste to control the amount of aluminum that partitions to the HLW glass stream. Developing technologies to minimize the impacts on the processing flowsheets and reducing the amounts of final waste forms produced are needed.

The overall goal of the project governing the work described in this report is to provide the technologies that will transformationally improve the performance planned treatment processes with regards to the processing of key waste constituents. The impact of these changes is anticipated to result in significant decreases in the duration of pretreatment operations. These improvements will result from the removal of bottlenecks within the existing planned operation and by shifting the burden for performance to new technology systems.

Currently, cross-flow filtration is deployed at both the Hanford Tank Waste Treatment and Immobilization Plant (WTP) and the Savannah River Site (SRS). However, there is a lack of understanding of the fouling mechanisms of these filters that prevent development of a strategy for determining the cleaning or backpulsing requirements for these facilities. The frequency of cleaning significantly impacts both process throughput as well as the sodium demand (through acid neutralization). Development of the cleaning/backpulsing requirements will produce much more efficient operations for both WTP and SRS, thereby significantly increasing throughput by limiting cleaning cycles. The scope of the research presented in the current report is to develop the understanding of filter fouling to allow development of this cleaning/backpulsing strategy.

This document completes the requirements of Milestone 2-4, Final Report of FY10 Testing, discussed in the scope of work outlined in the EM31 task plan WP-2.3.6-2010-1. The focus of task WP-2.3.6 is to improve the U.S. Department of Energy's (DOE's) understanding of filtration operations for HLW to improve filtration and cleaning efficiencies, thereby increasing process throughput and reducing the $\mathrm{Na}$ demand (through acid neutralization). Developing the cleaning/backpulsing requirements will produce much more efficient operations for both the WTP and SRS, thereby significantly increasing throughput by limiting cleaning cycles. The scope of this work is to develop the understanding of filter fouling to allow developing this cleaning/backpulsing strategy.

\subsection{Background}

The WTP Pretreatment Facility (PTF) will use cross-flow filtration to assist in HLW remediation efforts at the Hanford Site in Richland, Washington. General filtration operations can use dead-end or cross-flow filtration configurations. The two configurations differ in the direction of bulk slurry/solution flow relative to the surface of the filter. The two configurations, applied to the filtration of slurry, are 
shown in Figure 1.1. In dead-end filtration, slurry flow is normal to the filter membrane. Solids retained by the membrane stay on the membrane surface, forming a filter cake that grows proportionally to the volume filtered. The build-up of solids increases the resistance to permeate flow with time. To counteract the build-up of solids cake and to maintain constant permeate flow, increasing energy (or applied transmembrane pressure [TMP]) is required for dead-end filtration. In cross-flow filtration, slurry flow is tangential to the filter membrane. Like dead-end filtration, solids retained by the filter can remain on the filter surface; however, cake build-up is limited by the flow of slurry against the filter membrane, which can re-entrain solids and limit the growth of filter cake. Under certain operational conditions, it is possible to prevent the formation of a filter cake entirely (Bacchin et al. 2006).

A

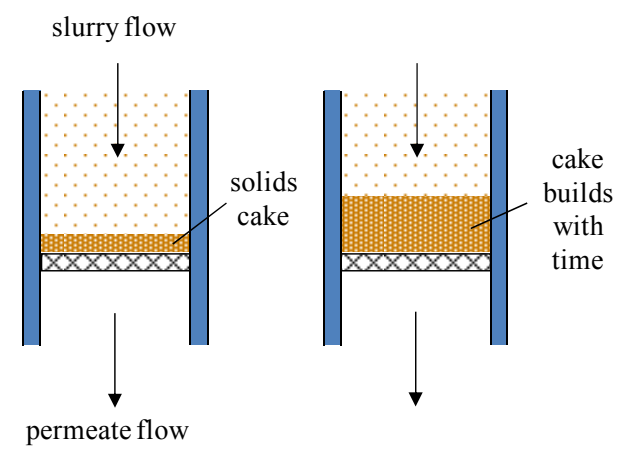

dead-end filtration
B
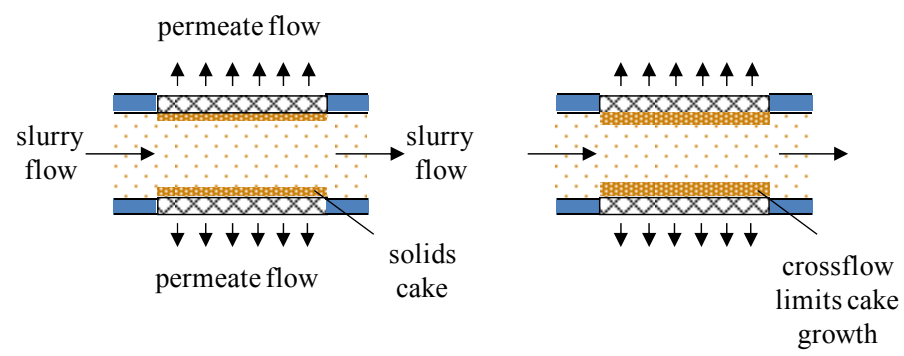

Figure 1.1. Dead-End and Cross-flow Filtration Configurations

Cross-flow filtration is one of the baseline separation technologies that are to be implemented for several treatment programs for high-level tank waste at the Hanford site. While the most well known of these is the PTF at WTP, it is a major component for other treatment programs being investigated by the Advance Remediation Technology (ART) program. WTP plant operations intend to use cross-flow filtration for waste solid/liquid separations. WTP operations will process waste slurries that contain colloidal particles, non-colloidal $(>1 \mu \mathrm{m})$ particles, and dissolved solutes. Selecting cross-flow geometry minimizes the build-up of solids on the surface of the filter elements and the fouling of those filter elements. In addition, filtration operations shall employ sintered stainless steel filters fabricated by the Mott Corporation (Farmington, CT) with a filter grade of 0.1. Parsons, along with Pacific Northwest National Laboratory (PNNL), is investigating the uses of similar filtrations as part of a Near Tank Treatment System (NTTS) that would process HLW waste similar to WTP PTF, but process waste continuously instead of using a batch process.

Despite the use of the cross-flow configuration for high-level waste filtration operations, sustained waste filtration operations and waste throughput are still expected to be challenged by fouling of the filter elements during HLW treatment operations. Specifically, the fouling dynamics of the cross-flow filters employed for high-level wastes, especially those at the Hanford site, are still not completely understood. This lack of understanding derives from the complex nature of the waste feeds, with waste feeds for each tank (or small tank groupings) typically having a unique chemical composition and physical properties. Even for a given waste storage tank, the waste feed is heterogeneous in both chemical constituents (which include both insoluble mineral phases, precipitated salts, and a $\sim 1$ - to 10-M concentration of dissolved species) and particle size $(\sim 0.2 \mu \mathrm{m}$ to $300 \mu \mathrm{m})$. Finally, understanding WTP filtration fouling dynamics 
is further complicated by the numerous processes that the waste feed will be subjected to (including as-received dewatering, dewatered feed caustic leaching, post-leach washing, etc.), which fundamentally alters both waste chemistry and particle size.

Planned maintenance activities to restore filter performance include both backpulsing of (i.e., flow reversal through) the filters and acid cleaning operations. Despite having the means to restore filter performance, the rate of waste filtration is expected to limit the rate of waste treatment. In addition, the long-term impact of processes such as filter backpulsing on filter performance is not well understood. Previous assessments of engineering-scale, WTP filtration operations indicate that backpulsing provides only temporary improvement in filter flux and that multiple backpulses may cause long-term degradation of filter performance (Daniel et al. 2009b).

\subsection{Need for Additional Fouling and Chemical Cleaning Studies}

The rate of permeate production achieved during filtration of HLW is key to the throughput of HLW dewatering, leaching, and washing operations. Permeate rates are expected to decline as a result of fouling of the filter elements employed by solid particles in the waste. Despite extensive testing of actual waste and waste simulants, uncertainty with respect to the rate and extent of filter flux decline still exists. This uncertainty derives in part from the fact that most studies of filtration currently performed are of limited duration (ranging from 5 to 10 hours) relative to the durations expected for full-scale treatment operations (on the order of 100 hours). As a result, the behavior of filter flux at times relevant to plant-scale operations is unknown. While models have been developed to allow prediction of actual waste filter performance at long-time (such as Daniel et al. 2010b), the accuracy of these models at long filtration times has not been tested. This general lack of information on long-time filtration behavior limits attempts to optimize the operational conditions, such as axial velocity, transmembrane pressure, and backpulse frequency, to yield maximum process throughput.

To help overcome the challenges associated with filtration of HLW, numerous studies have been performed to understand how wastes from specific Hanford tanks or groupings of tanks affected filter performance, yielding a partial knowledge of how cross-flow filters planned for high-level waste remediation efforts foul. In one such study, post-caustic leach dewatering of waste was studied; the test results were cause for concern because the dewatering process took significantly longer than planned because of an unexpected and significant loss of filter performance (i.e., relative to that measured in bench-scale studies, see Daniel et al. 2009b). Should similar conditions exist in the full-scale process, then waste throughput will be significant hindered, and the timeline for and costs associated with completing remediation of the Hanford site will be significantly extended beyond current estimates.

For filtration efforts under the EM31 task plan WP-2.3.6-2010-1, a review of previous waste filtration studies by PNNL and those reported in peer-reviewed literature was undertaken to better understand the challenges underlying filtration HLW (including, but not limited to, Hanford wastes). The results of this literature review are reported in Daniel et al. (2010b). The main objective of this report was to identify research areas that would allow improvement of current PNNL models for filtration of HLW (like that proposed in Daniel et al. 2010a). To improve the predictive value of PNNL models, the literature review report recommended two areas of additional research: 
- Long-Time Filtration Dynamics - Study of long-time fouling dynamics would 1) help evaluate the existence of filtration steady-state and 2) determine the functionality of long-time filter decay on operational parameters such as axial velocity (AV) and TMP. Current studies (which only encompass up to $\sim 10$ hours of undisturbed filter time) do not fully define the mechanics by which flux decays. Pore blocking and cake formation are thought to dominate, but observed phenomenon such as the formation of protective cake layers that prevent depth fouling are not explained. A time progression of filtration resistance change is sought, whereby we can better define time-scales for particle-pore interactions, cake growth, and cake maturation.

- Evaluate the Impacts of Solution Chemistry on the Filtration Process - Analysis of Hanford tank waste particle size distributions (PSDs) indicates that the waste contains a submicron fraction of particles that can engage in particle-pore interactions during filtration. Because these particles are colloidal, surface phenomena (such as electrostatic repulsion) can play a significant role in how these particles interact with the filter medium and each other. It is recommended that the impact of changing solution chemistry on filter performance (both in terms of flux decay during continuous filtration and recovery during backpulsing) be studied to help form predictive models of changing filter performance throughout the pretreatment process.

Based on the recommendations in the literature review, a series of filter tests was proposed to examine 1) the long-time fouling dynamics of the Pretreatment Engineering Platform (PEP) filtration simulant used in integrated PEP testing (Guzman-Leong et al. 2009; Geeting et al. 2009), 2) the fouling dynamics of individual PEP components as a function of solution $\mathrm{pH}$ and ionic strength, and 3) the effectiveness of chemical cleaning with nitric and oxalic acids. While these areas of research inform model development, they also provide insight into the long-term sustainability of HLW treatment operations.

Issues of long-term filter dynamics and solution chemistry impacts are discussed extensively in the literature review (Daniel et al. 2010a), and as such, readers are referred to that document, and an in-depth discussion of these topics is not provided herein. In contrast, the literature does not extensively discuss chemical cleaning approaches in detail. For this reason, this topic is given some attention in the following paragraphs.

Membrane cleaning remains to a large extent an empirical art (Zeman and Zydney 1996) specific to the membrane material and suspension or slurry being tested. Few quantitative studies have been published comparing wide ranges of potential fouling and cleaning agents for specific membrane materials. This is certainly true of most nuclear waste filtration studies in the past, where cleaning was a necessary adjunct to the filtration tests. The present effort represents an attempt to more systematically capture filter cleaning data for the porous, sintered stainless-steel membrane material used in these tests.

Numerous waste and waste simulant filtration tests have been recently performed at PNNL to support resolution of potential issues associated with operation of the Hanford Waste Treatment Plant. A partial listing of recent filtration reports that outline these tests is given in Table 1.1. Many of the filtration tests described in these reports were followed by chemical cleaning operations designed to restore filter flux loss that could not be recovered by backpulsing or simple washing operations alone. The initial approach to filter cleaning was consistent with planned cleaning operations at WTP and was to use concentrated (2M) solution of nitric acid $\left(\mathrm{HNO}_{3}\right)$ to dissolve fouling agents that could not be removed by other means. 
In the early stages of simulant testing and in testing of actual Hanford wastes [e.g., in Russell et al. (2009) and Shimskey et al. (2009a)], it was discovered that the baseline nitric acid cleaning was insufficient to restore the clean water flux (CWF) to desired pre-test levels after the filter had been fouled with precipitated sludge solids. A review of a Savannah River National Laboratory (SRNL) report (Poirier and Fink 2002) on porous stainless steel filter cleaning led to the selection of oxalic acid as a cleaning agent. When applied to bench-scale filtration at PNNL, it was found that cleaning with $0.5 \mathrm{M}$ oxalic acid solutions leached much of the irreversible (i.e., not removable by backpulsing) foulants and resulted in CWF values comparable to pre-test-values, or at least a fivefold or more increase over the CWF achieved after nitric acid cleaning alone.

For the remainder of the simulant development work (Russell et al. 2009), oxalic acid was used as the sole cleaning agent in most cases. For each of the simulant tests, the oxalic chemical cleaning was sufficient to remove irreversible membrane fouling and return the filter to the benchmark starting condition for future tests. For the actual waste processing campaigns, the client-approved test instructions specified nitric acid cleaning. This was performed for the first several tests in the series, and the response of the membrane after nitric acid cleaning was comparable to that observed for simulant. There was some recovery of CWF, but not near the level of a clean filter or the level that was obtained after oxalic acid cleaning in the simulant tests. After the third actual waste campaign, oxalic acid cleaning was added to the post-test cleaning and high CWF values were obtained. Oxalic acid cleaning was performed for the remainder of the actual waste campaigns and was in general much more successful at removing irreversible fouling than nitric acid alone.

Table 1.1. Partial Listing of Reports with Filtration Testing that Supported Resolution of Issues Involving Filtration at WTP

\begin{tabular}{ll}
\hline \multicolumn{1}{c}{ PNNL Report } & \multicolumn{1}{c}{ Description } \\
\hline Lumetta et al. (2009) & Filtration testing of actual waste - bismuth phosphate sludge/saltcake \\
Edwards et al. (2009) & Filtration testing of actual waste - TBP sludge and AY102 waste \\
Fiskum et al. (2009) & Filtration testing of actual waste - Ferrocyanide (FeCN) sludge \\
Shimskey et al. (2009a) & Filtration testing of actual waste - (REDOX) sludge and S-Saltcake \\
Shimskey et al. (2009b) & Filtration testing of actual waste - PUREX and REDOX cladding wastes \\
Russell et al. (2009) & Development of a waste filtration simulant \\
Daniel et al. (2009a) & Testing of the filtration simulant developed in Russell et al. (2009) \\
Daniel et al. (2009b) & Scale-up testing of the filtration simulant at the Pretreatment Engineering Platform \\
Billing et al. (2009) & Parallel bench-scale filtration supporting scale-up studies at the Pretreatment \\
Kurath et al. (2009) & Engineering Platform \\
\hline
\end{tabular}

Several of the most important questions regarding chemical cleaning with respect to the future filtration operations for high-level waste are:

- When is chemical cleaning necessary? Another way of asking this question is to ask whether there is an optimal frequency for chemical cleaning. The value of the gain in throughput anticipated as a result of chemical cleaning must be evaluated against the following costs: 
- process downtime

- material cost of acid

- material cost of sodium hydroxide to neutralize spent acid

- waste disposal path

- operational risks.

- How clean is clean? It should be noted that in previous filtration studies at PNNL there has not been a well-defined threshold for what is considered an "acceptable" or "desirable" clean water flux. In general, the filter was considered clean if a 40 pounds per square inch differential (psid) TMP corrected clean water flux of $1 \mathrm{GPM} / \mathrm{ft}^{2}$ or greater was maintained over 15 -minutes of clean water filtration. However, this criterion does not have a rigorous experimental underpinning and in many cases was not followed consistently. Justification for why the $1 \mathrm{GPM} / \mathrm{ft}^{2}$ criterion is considered acceptable lies mostly in the fact that it provides a baseline clean flux that is much greater than the highest fluxes observed for low-solids $(\sim 5-\mathrm{wt} \%)$ waste slurries, which typically fall below $0.1 \mathrm{GPM} / \mathrm{ft}^{2}$.

As such, one objective of this work is to create a framework or decision matrix about what constitutes a "clean" filter. By correlating the extent of cleaning performed (via CWF values) with actual initial flux obtained within the process uncertainty, quantitative values for sufficient CWF may be suggested. Such values are somewhat of a theoretical abstraction, as the type of cleaning that can be employed at the bench scale to reach the quantitative threshold may not be practicable at larger scales. The answer to this question is contingent on the answer to the first question: whether or not chemical cleaning should be performed. If it is necessary or advantageous to clean, this second question is concerned with defining the success criteria.

As such, a subset of testing in the current report will focus on providing answers to these questions such that 1) the overall efficacy and value of the chemical filter cleaning process can be determined and 2) chemical cleaning operations can be optimized. This work performed represents a starting point for quantifying the effect of chemical cleaning for the materials tested. Due to the specific nature of membrane cleaning, extrapolation or correlation of the quantitative results to other simulants, membrane materials, and cleaning regimens must be taken with care. It is the authors' hope that the principles and metrics presented will guide future work in this area and as more data is collected, the quantitative aspects may be gradually extended.

\subsection{Task Objectives}

The overall goal of the EM31 WBS 2.3.6 "Filtration Understanding" program is to improve knowledge of what factors impact high-level waste filtration operations and to develop methods to increase filter throughput. The overall approach taken to achieve this goal is to:

- Review previous cross-flow testing at SRS, PNNL, and other peer review journals to direct testing activities for this task.

- Develop a predictive model that reflects the important physical mechanisms of fouling and clearing for use in determining effective filter cleaning strategies for a variety of feeds. 
- Develop simulants that can result in significant irreversible fouling, but also are representative of the types of materials that are present in HLW.

- Test these simulants in bench-scale equipment to identify the cleaning requirements for these feeds.

- Develop a cleaning strategy to optimize throughput while minimizing added Na.

- Validate the model and operational strategy with actual waste samples as appropriate.

As shown in Table 1.2, there are two main objectives for this task with respect to FY10 test activities. These are:

1. Literature Review. Perform a literature review of previous testing to support direction of testing activities and filtration model (Subtask 1).

2. Filtration Testing. Perform additional filtration testing to provide the underlying data for verification of a filtration model (Subtask 2).

Table 1.2. Task Objective Milestones

\begin{tabular}{cl}
\hline Task Objective & \multicolumn{1}{c}{ Milestones } \\
\hline Objective One: & Milestone 1-1: Issue Test Plan for FY10 Testing \\
Literature Review & Milestone 1-2: Draft Report of Literature Review \\
& Milestone 1-3: Final Report of Literature Review \\
& Milestone 2-1: Complete Series 1 Testing \\
Objective Two: & Milestone 2-2: Complete Series 2 Testing \\
Filtration Testing & Milestone 2-3: Complete Series 3 Testing \\
& Milestone 2-4: Final Report of FY10 Testing \\
\hline
\end{tabular}

The first task objective was completed with the issuance of PNNL-20023 (Daniel et al. 2010a) in November 2010. The report is a review of the available information regarding filtration performance with simulated and HLW from SRS and Hanford. This review summarizes prior filtration experience and focuses on filter fouling data for the various materials tested. A separate review for the SRS was authored by SRNL (Johnson and Duignan 2011).

The second task objective involved additional filtration tests to provide the underlying data for model validation provided in the initial draft literature report. These tests were performed using existing equipment and used simulants for various actual waste streams. Testing was of sufficient duration to provide estimates of long-term filtration performance. In addition, testing assessed the impact of feed variability on filtration behavior. In particular, tests were performed with simulants of varying chemical affinity for the filter membrane material. The testing program was split into three series of tests. The first series of tests provided data on long-term filtration performance using baseline equipment and simulants used from previous testing programs. The second series of tests focused on filtration testing on simulants of varying chemical affinity to the filter, defined by zeta potential measurements. The third series of tests were cleaning tests on the filter fouled with simulants known to present problems (e.g., iron sludge simulant). This report summarizes the results of these testing activities and updates to the initial filtration model presented in the literature report. 



\subsection{Quality Assurance}

In accordance with the Project Quality Assurance Plan (PQAP) for the Environmental Management (EM)-31 Support Project, this work was designated as Quality Level 3 (QL3). This designation is based on the fact that the work is exploratory (experimental) in nature and will not be used directly for the design or operation of vitrification facilities. As such, the work was performed in accordance with best laboratory practices, Nuclear Quality Assurance (NQA)-1, Subpart 4.2 - based, as indicated in work flows and subject areas of the PNNL "How do I...?" (HDI) standards-based management system. 



\subsection{Testing Approach}

As discussed in Section 1.3, one of the main objectives of task WP-2.3.6 is to perform filtration testing to provide the underlying data for verification of PNNL's existing filtration model (Daniel et al. $2010 \mathrm{~b}$ ). To this end, three separate series of filtration testing were conducted to provide insight into dynamics of filter fouling and the efficacy of flux-recover operations, including both backpulsing of the filter and chemical cleaning regimens. The three tests series are:

- Series 1, Long Term Fouling Tests - examined flux decline when filtering the PEP waste simulant slurry for long periods of time without backpulsing. Previous filtration testing (e.g., Daniel et al. 2009b) has only studied filter flux dynamics over relatively brief periods of operation (4-12 hours) with respect to expected filtration operation times at WTP (on the order of 100 hours). Analysis of previous filtration campaigns evidenced continued flux decline over the entire duration of filtration testing, and as such, the existence of a filtration steady-state could not be evaluated. As such, there is currently a need to evaluate filtration behavior at times longer than currently tested ( $\sim 10$ hours) to allow development of better predictive models and optimization of flux recovery operations in PEP. The aim of Series 1 testing was to answer this need. Series 1 testing examined filter flux decline over 100 hours of continuous undisturbed (i.e., non-backpulsed) filter operation. The reversibility of this flux decline with respect to backpulsing was then determined by backpulsing the filter.

- Series 2, Component Fouling Tests - determined the filtration behavior of the major components that comprise the PEP simulant. The components tested include boehmite $(\mathrm{AlOOH})$, gibbsite $\left[\mathrm{Al}(\mathrm{OH})_{3}\right]$, iron (III) oxy-hydroxide $(\mathrm{FeOOH})$, and Chromium (III) oxy-hydroxide $(\mathrm{CrOOH})$. Previous filtration studies for WTP have examined the filtration behavior of complex multicomponent wastes and waste simulants. Because of the complexity of the slurries tested, there is still uncertainty about the mechanisms underlying filter fouling behaviors for WTP waste and waste simulants. For example, the current filtration behavior does not allow one to determine if observed flux declines are caused by a single component or by the mixture of components. Likewise, it has been impossible to evaluate, even for waste simulants like the PEP simulant, if fouling is driven by particle-particle interactions (cake formation), particle-filter interactions (depth fouling and/or pore blocking), or some combination thereof. For this reason, it is desirable to study the filtration behavior of individual waste components to allow identification of any major foulant particle species. The nature of particle-particle and particle-medium interactions can be evaluated by changing suspending phase chemistry. By examining changes in the filtration behavior with changing chemical environment, insight about the filtration behavior under both normal operating conditions and changing chemical conditions (like those experienced during waste washing operations or filter cleaning) operations can be provided. The goal of Series 2 testing was to determine the major fouling components in the PEP simulant waste slurry and to gain insight into role of particle-particle and particle-filter interactions in the overall flux behavior over a range of operating $\mathrm{pH}$.

- Series 3, Cleaning Tests and Filter Evaluation - determined the efficacy of filter flux restoration through chemical cleaning operations. Previous filtration testing has evidenced a decline in filter performance that cannot be recovered through backpulsing operations alone for both actual Hanford tank waste and waste simulant slurries. Historically, chemical cleaning operations in PNNL waste filtration studies have used solutions of oxalic and nitric acid. While these operations have generally been viewed as being effective at restoring flux, there has been no systematic evaluation of the effectiveness of either oxalic or nitric cleaning. The goal of Series 3 testing was to provide systematic testing of flux restoration by nitric and oxalic cleaning regimens. This testing will provide a comprehensive picture of chemical cleaning efficacy. 


\subsection{Series 1 Testing: Long-Term Fouling Tests}

Long-term fouling tests examined the decline of filter flux with time to determine 1) the existence of a steady-state flux and 2) the value of flux at steady-state should the steady-state flux exist. Tests used as-prepared PEP simulant slurry developed for Integrated Tests A and B (see Guzman-Leong et al. 2009; Geeting et al. 2009; and Kurath et al. 2009). Tests studied long-time fouling dynamics and flux decline by filtering the as-prepared PEP simulant slurry in continuous-recycle filtration (where all permeate collected was immediately and continuously returned to the slurry reservoir) at $\sim 5$-wt $\%$ undissolved solids (UDS) and at various AV and TMP setpoint combinations. To eliminate the need to account for filter history, each test started with a filter element that was cleaned in nitric and oxalic acid solutions. Each test examined only one AV and TMP combination. For each test, four test segments were planned. These were:

- Segment 1: 100-hour period of continuous recycle filtration at select AV/TMP - the decline in filter flux during continuous recycle filtration of the $\sim 5-\mathrm{wt} \%$ UDS PEP simulant slurry was monitored for 100 hours to assess the existence of a steady-state filter flux. During this time, the filter was not backpulsed, and adjustments to AV/TMP setpoints limited to only what is necessary to maintain the filter setpoints.

- Segment 2: Dewatering of the slurry - monitoring the decline in filter flux during dewatering of the slurry from the initial concentration ( $\sim 5-\mathrm{wt} \%$ UDS) to concentrations in excess of $15-\mathrm{wt} \%$ UDS.

- Segment 3: Dilution of the slurry - monitoring the change in filter flux as permeate removed during the previous segment is returned to the slurry (i.e., the slurry is "rewatered").

- Segment 4: Backpulse and flux decline - the rewatered slurry is subjected to one or more backpulses to measure the reversible fouling that occurred during the first three test segments. Flux decline following the backpulse was monitored briefly (for approximately 3 to 4 hours) during continuous recycle filtration of the $5-\mathrm{wt} \%$ rewatered slurry.

In actual testing, Segments 2 and 3 were not performed because in all cases the filter flux after 100 hours of testing was below or near $10 \mathrm{~mL} / \mathrm{min}$ (which is the lower measuring limit of the filtrate flow meter). Dewatering of the slurry, which was expected to further lower flux, would have likely generated data below the calibration range of the flow meter, and as such, had a low probability of generating useable data. Moreover, identifying key points in the dewatering curve (such as the dewatering knee) become difficult at low permeate rates because of process noise. For these reasons, only Segments 1 and 4 were attempted for actual testing.

All long-time fouling tests employed the cell unit filter (CUF) test system with a 2-foot sintered stainless steel filter element (Mott Grade 0.1). Five long-term fouling tests will be performed. These tests examined the influence of axial velocity and transmembrane pressure setpoints on long-term filter flux fouling dynamics. Each test examined a single AV/TMP combination. The baseline test matrix for Series 1 testing is outlined in Table 3.1. All test segments attempted (i.e., Segments 1 and 4) employed the AV/TMP combinations listed in Table 3.1. 
Table 3.1. AV/TMP Matrix for Series 1 Testing

\begin{tabular}{ccc}
\hline Test ID & $\begin{array}{c}\text { AV } \\
{[\mathrm{ft} / \mathrm{s}]}\end{array}$ & $\begin{array}{c}\text { TMP } \\
{[\mathrm{psid}]}\end{array}$ \\
\hline S1T1 & 15 & 40 \\
S1T2 & 17 & 40 \\
S1T3 & 13 & 40 \\
S1T4 & 15 & 60 \\
S1T5 & 15 & 20 \\
\hline
\end{tabular}

\subsection{Series 2 Testing: Component Fouling Tests}

Component fouling tests examined the filtration behavior of individual simulant components and the impact of both particle and filter membrane surface charge on the rate of filter flux decline observed during continuous recycle filtration and during sequential backpulsing of the filter. The tests studied particle deposition on membranes at low particulate concentrations over a range of suspending phase $\mathrm{pH}$ at a controlled ionic strength. Single component suspensions of the following materials were employed:

- Boehmite $\mathrm{AOH} 20$

- Boehmite AOH180e

- Iron (III) Oxy-hydroxide, $\mathrm{FeOOH}$

- Chromium (III) Oxy-hydroxide, $\mathrm{CrOOH}$

The two boehmite solids (AOH20 and AOH180e) differ only in particle size distribution. AOH20 solids are coarse relative to AOH180e solids. The four single component suspensions were chosen because they are present in the Hanford tank waste and boehmite, $\mathrm{FeOOH}$ and $\mathrm{CrOOH}$ are all significant components of the PEP simulant.

To allow examination of particle-membrane interactions, suspensions prepared using these materials had low particle concentration ( 0.1 -vol\% particles) to facilitate membrane fouling and to avoid substantial cake formation. The zeta potential of each suspension prepared for testing was characterized as a function of $\mathrm{pH}$ at a controlled ionic strength. Filtration experiments examined the steady-state flux decline (i.e., fouling) experienced when filtering these $0.1-\mathrm{vol} \%$ suspensions (refer to Table 3.3 for the list of tests performed) using the CUF test system and a 2-foot sintered stainless steel filter element (Mott Grade 0.1). Each fouling test consisted of at least five test segments (refer to Table 3.2 for more information about the parameters used):

1. Segment 1: Initial period of recycle-filtration at $\mathrm{pH} \sim 10$ - the suspension was filtered in recycle-mode (where all permeate collected is immediately returned to the suspension reservoir) for a set amount of time (e.g., 12 hours) without backpulsing of the filter element.

2. Segment 2: Backpulsed recycle-filtration at $\mathrm{pH} \sim 10$ - the suspension was filtered in recycle-mode and the filter element is periodically backpulsed (e.g., every 30 minutes).

3. Segment 3: Second period of recycle-filtration at $\mathrm{pH} \sim 10-$ the suspension was filtered in recycle-mode for a set period of time without backpulsing.

4. Segment 4: Recycle-filtration during titration of the suspension - the suspension was filtered and, at set intervals (e.g., 1 hour), a step decrease in the $\mathrm{pH}$ was effected by titration with $0.01 \mathrm{M} \mathrm{HNO}_{3}$ solution until a $\mathrm{pH}$ of 3 was achieved. The $\mathrm{pH}$ was varied from 10 to 3 to encompass the isoelectric 
point of each of the suspensions (typically between $\mathrm{pH}$ of $5-9$ ); this $\mathrm{pH}$ range is consistent with that used in literature studies such as Huisman et al. (1998).

5. Segment 5: Fouled filter water flux - the test suspension employed in segments 1 to 4 was drained from the system, and the system was flushed with $5 \mathrm{~L}$ of deionized water and drained again. The system was then filled with $\sim 5-\mathrm{L} 0.01 \mathrm{M} \mathrm{NaOH}$ solution and operated in continuous recycle mode (at fixed AV and TMP) for 2 hours. During this time, the flux was monitored for decline.

Table 3.2. Common Parameters Used for Series 2 Filtration Testing

\begin{tabular}{|c|c|}
\hline Parameter & Value \\
\hline Suspension particle concentration & $0.1 \mathrm{vol} \%$ \\
\hline Background electrolyte $\left(\mathrm{NaNO}_{3}\right)$ concentration & $0.05 \mathrm{M}$ \\
\hline $\begin{array}{l}\text { Clean water flux measurement (before testing): } \\
\text { Axial velocity (AV): } \\
\text { Transmembrane pressure (TMP): } \\
\text { Duration: }\end{array}$ & $\begin{array}{l}10 \mathrm{ft} / \mathrm{s} * \\
10 \mathrm{psid} \\
2 \text { hours }\end{array}$ \\
\hline $\begin{array}{l}\text { Segment 1: } \\
\text { Axial velocity (AV): } \\
\text { Transmembrane pressure (TMP): } \\
\text { Duration: }\end{array}$ & $\begin{array}{l}15 \mathrm{ft} / \mathrm{s} \\
40 \mathrm{psid} \\
12 \text { hours }\end{array}$ \\
\hline $\begin{array}{l}\text { Segment 2: } \\
\text { Axial velocity (AV): } \\
\text { Transmembrane pressure (TMP): } \\
\text { Duration: } \\
\text { Backpulse frequency: }\end{array}$ & $\begin{array}{l}15 \mathrm{ft} / \mathrm{s} \\
40 \mathrm{psid} \\
12 \text { hours } \\
1 \text { backpulse every } 30 \text { minutes }\end{array}$ \\
\hline $\begin{array}{l}\text { Segment 3: } \\
\text { Axial velocity (AV): } \\
\text { Transmembrane pressure (TMP): } \\
\text { Duration: }\end{array}$ & $\begin{array}{l}15 \mathrm{ft} / \mathrm{s} \\
40 \mathrm{psid} \\
12 \text { hours }\end{array}$ \\
\hline $\begin{array}{l}\text { Segment 4: } \\
\text { Axial velocity (AV): } \\
\text { Transmembrane pressure (TMP): } \\
\text { Total Duration: } \\
\text { Duration of each pH setpoint } \\
\text { pH Step Change: } \\
\text { Backpulse frequency: } \\
\text { Backpulse timing: }\end{array}$ & $\begin{array}{l}15 \mathrm{ft} / \mathrm{s} \\
40 \mathrm{psid} \\
16 \text { hours } \\
2 \text { hours } \\
1 \\
1 \text { backpulse at each } \mathrm{pH} \text { setpoint } \\
1 \text { hour after } \mathrm{pH} \text { step change }\end{array}$ \\
\hline $\begin{array}{l}\text { Segment 5: } \\
\text { Axial velocity (AV): } \\
\text { Transmembrane pressure (TMP): } \\
\text { Duration: }\end{array}$ & $\begin{array}{l}10 \mathrm{ft} / \mathrm{s} * \\
10 \mathrm{psid} \\
2 \text { hours }\end{array}$ \\
\hline $\begin{array}{l}\text { * Initial planning called for testing at } 15 \mathrm{ft} / \mathrm{s} \text {, } \\
\text { capacity and overheating concerns. }\end{array}$ & was reduced to $10 \mathrm{ft} / \mathrm{s}$ because of pump \\
\hline
\end{tabular}

Table 3.3. Series 2 Test Matrix for the Five Baseline Tests

\begin{tabular}{llc}
\hline Test ID & Particle System & $\begin{array}{c}\text { Background } \\
\text { Electrolyte }\end{array}$ \\
\hline S2T1 & Boehmite AOH20 & $0.05 \mathrm{M} \mathrm{NaNO}_{3}$ \\
S2T2 & FeOOH & $0.10 \mathrm{M} \mathrm{NaNO}_{3}$ \\
S2T4 & Boehmite AOH180e & $0.05 \mathrm{M} \mathrm{NaNO}_{3}$ \\
S2T6 & CrOOH & $0.05 \mathrm{M} \mathrm{NaNO}_{3}$ \\
S2T8 & FeOOH & $0.05 \mathrm{M} \mathrm{NaNO}_{3}$ \\
\hline
\end{tabular}


Segments $1-3$ were conducted in the same way that CUF testing has been performed for filtration scaling tests with the PEP simulant (Daniel et al. 2009b), providing a simple comparison between the full simulant flux and the individual, dilute component flux. Segment 4 was a series of 2 hour segments, each at a different $\mathrm{pH}$, conducted to observe the effect of $\mathrm{pH}$ on particle-membrane interactions. The suspension was titrated to the target $\mathrm{pH}$, filtered in recycle mode for 1 hour, backpulsed, and then filtered for another hour before proceeding to the next $\mathrm{pH}$ increment. In this way, the $\mathrm{pH}$ decreased in steps from 10 to 3 over a period of 16 hours.

In some tests, the $\mathrm{pH}$ suspension was restored to a $\mathrm{pH}$ above the isoelectric point (see Section 4.2.5) by addition of $0.01 \mathrm{M} \mathrm{NaOH}$ solution. $\mathrm{pH}$ reversal is done to observe the degree of reversibility associated with changes in flux behavior caused by lowering of $\mathrm{pH}$. During all titrations, the suspending phase ionic strength was maintained by the presence of a background electrolyte in the test and titrating solutions (typically a baseline $0.05 \mathrm{M} \mathrm{NaNO}_{3}$ ). A concentration of $0.05 \mathrm{M} \mathrm{NaNO}_{3}$ represents a balance between keeping the ionic strength relatively constant while still being able to manipulate the $\mathrm{pH}$ using a reasonable amount of titrant. Note that for one suspension $(\mathrm{FeOOH})$ the effect of doubling the ionic strength (using $0.10 \mathrm{M} \mathrm{NaNO}_{3}$ ) was tested to compare to the $0.05 \mathrm{M}$ results.

Prior to the start of each filtration experiment, the CUF test system was chemically cleaned with solutions of nitric and/or oxalic acid as necessary to restore flux to greater than $1 \mathrm{GPM} / \mathrm{ft}^{2}$. After cleaning the filter element, a measurement of the clean water flux ${ }^{1}$ (and any decline in that flux) was made at the same AV and TMP and over 2 hours to allow direct comparison to the post-test clean water flux measured in test segment 5 . While these clean water flux measurements do not directly provide experimental information for Series 2, they do provide a more extensive data set for Series 3 testing data analysis.

Table 3.2 and Table 3.3 outline the test conditions for each of the baseline particle systems. In particular, Table 3.2 presents operating parameters common to all tests. Table 3.3 presents the test matrix of particle systems and $\mathrm{pH}$ for the six baseline Series 2 tests.

Series 2 testing employed the CUF test system with a 2-foot Mott Grade 0.1 sintered stainless steel filter element. Zeta potential of the particles was measured using a Brookhaven ZetaPALS. The particle size distribution of the suspension was characterized either using a Malvern Mastersizer 2000 where the majority of particles are greater than $1 \mu \mathrm{m}$ or the Brookhaven ZetaPALS where the majority of particles are less than $1 \mu \mathrm{m}$.

\subsection{Series 3 Testing: Cleaning Tests and Filter Evaluation}

Filter evaluation tests to determine the efficacy of cleaning included both standalone filtration tests with slurries of strontium carbonate and filtration tests with the PEP simulant and simulant components (described in Section 6.1.1) incorporated into Series 1 and Series 2 testing. Because of the integration of multiple simulant slurries in testing, a single cleaning approach was not followed for all testing. Rather, the cleaning approach was tailored to the series (e.g., Series 1) to which set of cleaning evaluations was tied. The general approached use for each series testing is described in the following subsection.

\footnotetext{
1 Note that the CWF was measured with $0.01 \mathrm{M} \mathrm{NaOH}$, which has a $\mathrm{pH}=12$.
} 


\subsubsection{Cleaning Tests Associated with Series 1}

Cleaning tests associated with Series 1 studied cleaning after filtration with the PEP simulant described in Section 5.1. The cleaning performed during this was analogous to the standard cleaning regimen developed for this simulant material during historical testing (see Section 1.2). The basic steps conducted for each Series 1 cleaning test are given Figure 3.1.

Cleaning tests both preceded and followed the main portion of Series 1 testing (as outlined in Section 3.1). Before each Series 1 test, a measurement of pre-test CWF was taken with a solution of $0.01 \mathrm{M} \mathrm{NaOH}$. For this initial CWF test, filter flux behavior over 10-20 minutes at three TMP setpoints $(10,15$, and $20 \mathrm{psid})$ and an $\mathrm{AV}$ of $\sim 10 \mathrm{ft} / \mathrm{s}$ was recorded. A single backpulse was performed at the start of each TMP setpoint to remove any trace solids remaining in the system from the surface of the filter element. This initial CWF measurement provides a snapshot of baseline filter performance before each Series 1 test. After each Series 1 test, two CWF tests were conducted to establish flux before and after acid cleaning. These final CWF measurements are integrated into the normal filter cleaning operations, including rinsing and acid washing, as described in the following paragraph.

The first step in each post-Series 1 cleaning test was to drain the spent PEP simulant slurry from the CUF. Next, the CUF was rinsed with three separate volumes of $0.01 \mathrm{M} \mathrm{NaOH}$ solution. The rinse solution was completely drained from the filter system, including the shell side of the filter element, the backpulse chamber, and all associated permeate piping, after each rinse operation. Once rinsing of the filter system was complete, a CWF test was performed to provide a measure of flux after simple rinsing and before the next step in the cleaning process, which was cleaning with oxalic acid. This CWF test studied filtration of 2.5-L of $0.01 \mathrm{M} \mathrm{NaOH}$ solution at three target TMPs (10, 20, and $30 \mathrm{psid})$ and an AV of $\sim 10 \mathrm{ft} / \mathrm{s}$ with a backpulse at the start of each TMP setpoint. ${ }^{2}$ The filter flux was measured for 10-20 minutes at each condition. After this pre-acid clean CWF test was complete, a sample of the $0.01 \mathrm{M} \mathrm{NaOH}$ solution was collected from the slurry reservoir for analysis. The system was then completely drained in preparation for acid cleaning.

To acid clean the CUF filter system, approximately 2-L of $0.5 \mathrm{M}$ oxalic acid (either fresh or previously used) was introduced to the slurry reservoir and allowed to circulate through the slurry recirculation loop and back and forth across the filter element by means of filling and discharging the backpulse chamber. During this time, the permeate flow valve remained closed to isolate the flow meters from unnecessary exposure to the acid and to minimize the number of acid-wetted surfaces that must later be rinsed. The oxalic acid was allowed to circulate for 30 minutes and was then drained from the system. All acid-wetted surfaces were rinsed with deionized (DI) water using the chemical feed funnel on the backpulse chamber. ${ }^{3}$ The slurry reservoir was rinsed with a plastic squeeze bottle filled with DI water.

2 The post-test CWF was measured at higher TMPs (10, 20, and 30 psid vs. 10, 15, and 20 psid) for two reasons: 1) to keep the flux low enough to be read accurately by the permeate flow meter, and 2) to remain consistent with previous PNNL practice, where a higher TMP was required post-test because the filter is fouled.

3 By using the chemical feed port on the backpulse chamber, one may rinse the wetted surfaces with a minimum volume of DI water per rinse since it is not necessary to operate the CUF pump, for which 1-2 L of rinse water are required. It is still necessary to perform at least one rinse with the pump on; the other preliminary rinses simply enhance the effectiveness of the DI rinse. 


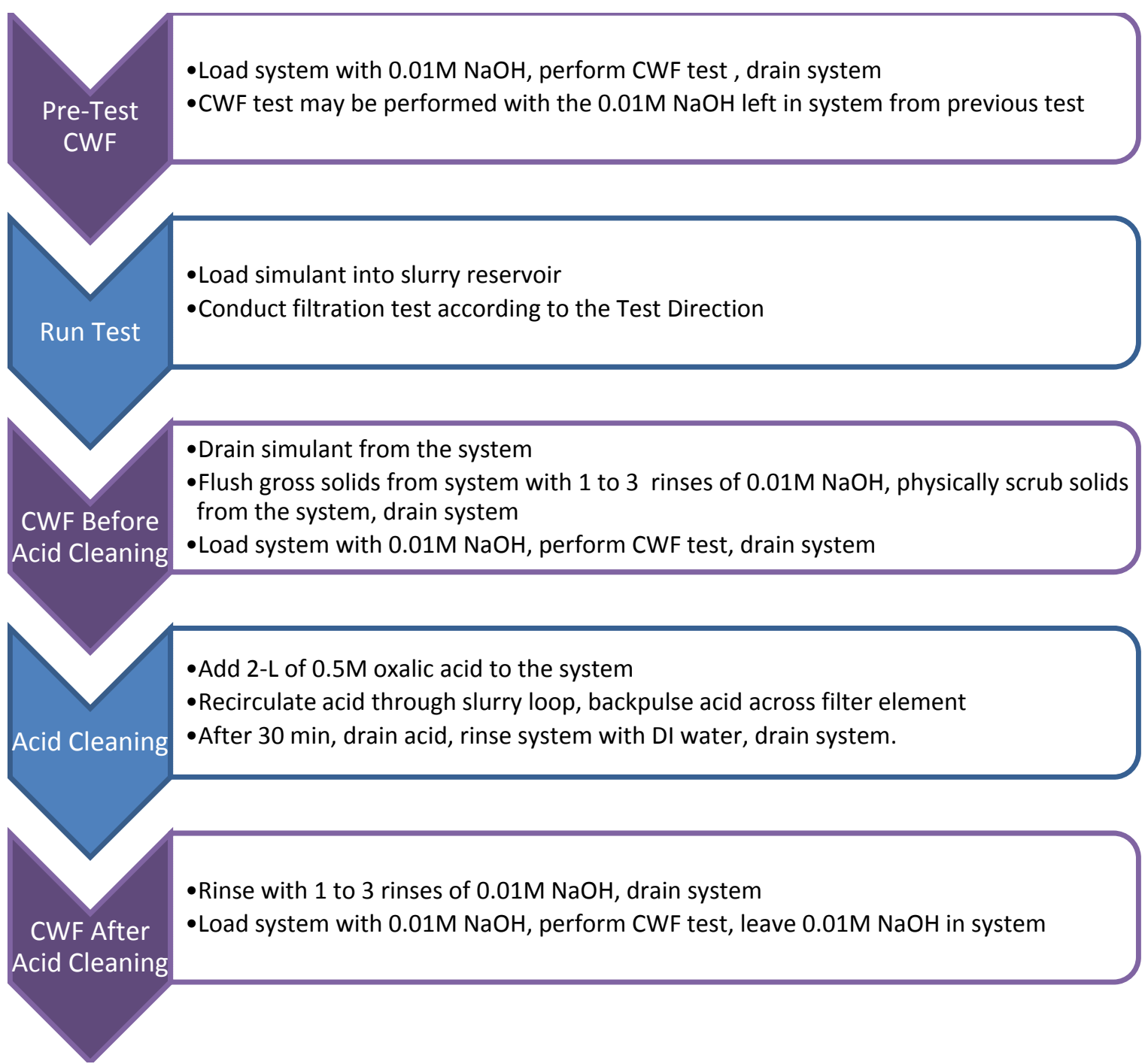

Figure 3.1. Overview of Cleaning Steps and CWF Tests During Series 1 Testing

Once the residual oxalic acid had been rinsed from the system, a rinse of $\sim 2 \mathrm{~L}$ of $0.01 \mathrm{M} \mathrm{NaOH}$ was added to the slurry reservoir and allowed to recirculate through the system. The backpulse chamber was filled and discharged several times. The rinse volume was completely drained and one or more similar rinses were performed. To aid the removal of trace suspended solids, the system was completely drained, and the filter element was flushed with $0.01 \mathrm{M} \mathrm{NaOH}$ via the chemical feed funnel in a sequence of small-volume rinses. Each $\sim 200-\mathrm{mL}$ rinse volume was drained from the slurry sampling valve and from the shell side of the filter element before the next rinse volume was delivered as an attempt to avoid infinite dilution of suspended particles through back-mixing of rinse volumes. As each rinse volume was removed from the system, the suspended particles associated with that rinse volume were removed.

After up to 10 rinses were delivered in this fashion, the slurry reservoir was filled with $2.5 \mathrm{~L}$ of $0.01 \mathrm{M} \mathrm{NaOH}$, and a post-acid cleaning CWF test was conducted to evaluate the effectiveness of the cleaning steps described above. For this CWF test, the system was run in recycle mode at three target TMPs (10, 15, and $20 \mathrm{psid})$ and an AV of $10 \mathrm{ft} / \mathrm{s}$ for approximately 10-20 minutes at each pressure with a single backpulse at the start of each TMP setpoint. 


\subsubsection{Cleaning Tests Associated with Series 2}

As discussed in preceding sections, cleaning tests associated with Series 2 testing involved an initial CWF measurement on the clean filter before each Series 2 test and a final CWF tests on the post-test filter after rinsing with DI water but before any chemical cleaning. Initial CWF measurements were conducted as part of Series 2 pre-test operations, while final CWF measurements were associated with Series 2 test segment 5 (see Section 3.2). Both CWF tests were run at an axial velocity of $10 \mathrm{ft} / \mathrm{s}$ and a single TMP setpoint of 10 psid. In addition, CWF data were recorded for 2 hours rather than the typical 10-20 minute test segments employed historically. Analogous to the extended duration of filtration operations for the PEP simulant tests in Series 1, these extended CWF tests helped define the fouling behavior beyond the traditional time frames, in some cases either confirming rapid decay to a relatively stable level or revealing a delayed decline in CWF that would not have been anticipated or discovered in the shorter tests.

For cleaning tests associated with component testing (Series 2 filtration), the cleaning process employed between tests was component dependent. Because the CUF filter element was replaced before Series 2 testing (see Section 4.1.1), initial preparation of the filter for Series 2 testing involved conditioning the filter with a strontium carbonate $\left(\mathrm{SrCO}_{3}\right)$ slurry and acid cleaning with nitric acid. Beyond this, the following clean operations were applied as Series 2 progressed (in order):

- S2T1, coarse boehmite - no post-test cleaning

- S2T4, fine boehmite - post-test cleaning with $2 \mathrm{M}$ nitric acid

- S2T8, FeOOH (low ionic strength) - post-test cleaning with $0.5 \mathrm{M}$ oxalic acid

- $\mathrm{S} 2 \mathrm{~T} 6, \mathrm{CrOOH}$ - post-test cleaning with $0.5 \mathrm{M}$ oxalic acid

- $\mathrm{S} 2 \mathrm{~T} 2, \mathrm{FeOOH}$ (high ionic strength) - post-test cleaning associated with strontium carbonate slurry testing (described in Section 3.3.3).

The applied post-test cleaning strategies were selected based on the final post-test CWF. Lower post-test CWF required more effective cleaning agents, i.e., nitric acid (reasonably effective) or oxalic acid (most effective).

\subsubsection{Strontium Carbonation Cleaning Tests}

Following the last test in Series 2, filtration of a $0.1-\mathrm{vol} \% \mathrm{FeOOH}$ suspension, a sequential cleaning test series incorporating strontium carbonate $\left(\mathrm{SrCO}_{3}\right)$ baseline tests was performed. It has been suggested in previous work that the standard $0.35 \mathrm{M} \mathrm{SrCO}_{3}$ solution [outlined in Appendix A of Geeting et al. (2003)] effectively masks the trace particles or impurities that cause rapid decay in clean water flux tests, and thereby provides a more reliable baseline indication of the filter resistance. The $\mathrm{SrCO}_{3}$ cleaning test proceeded according to the steps outlined in Table 3.4. Testing with $\mathrm{SrCO}_{3}$ slurry incorporates not only $\mathrm{SrCO}_{3}$ flux measurements but two CWF flux tests as well. 
Table 3.4. Test Outline for $\mathrm{SrCO}_{3}$ Cleaning Tests

\begin{tabular}{|c|c|c|}
\hline Step & Description & Operating Conditions \\
\hline 1 & $\begin{array}{l}\text { The } 0.1 \text { vol } \% \text { FeOOH slurry from S2T2 was drained from the system, and the } \\
\text { system was rinsed with several volumes of } 0.01 \mathrm{M} \mathrm{NaOH} \text { solution. }\end{array}$ & N/A \\
\hline 2 & $\begin{array}{l}\text { An initial CWF test was performed by loading } 2.5 \mathrm{~L} \text { of } 0.01 \mathrm{M} \mathrm{NaOH} \text { solution } \\
\text { into the CUF and filtering this solution in continuous recycle mode. After the } \\
\text { test, the } 0.01 \mathrm{M} \mathrm{NaOH} \text { solution was drained from the filtration system. }\end{array}$ & $\begin{array}{l}\text { AV: } 11 \mathrm{ft} / \mathrm{s} \\
\text { TMP: } 10 \mathrm{psid} \\
\text { Duration: } 2 \text { hours }\end{array}$ \\
\hline 3 & $\begin{array}{l}\text { An initial } \mathrm{SrCO}_{3} \text { (Test \#1) was conducted. Approximately } 8 \mathrm{~L} \text { of } \sim 0.35 \mathrm{M} \\
\mathrm{SrCO}_{3} \text { slurry was added to the slurry reservoir. The system was backpulsed } \\
\text { once and the slurry was filtered in recycle mode. After the test, the } \mathrm{SrCO}_{3} \text { was } \\
\text { drained from the system, the system was rinsed with } 2 \mathrm{~L} \text { of } 0.01 \mathrm{M} \mathrm{NaOH} \\
\text { solution. }\end{array}$ & $\begin{array}{l}\text { AV: } 13 \mathrm{ft} / \mathrm{s} \\
\text { TMP: } 40 \mathrm{psid} \\
\text { Duration: } 4 \text { hours }\end{array}$ \\
\hline 4 & $\begin{array}{l}\text { The CUF system was cleaned with nitric acid. Approximately 2-L of 2-M nitric } \\
\text { acid solution was added to the slurry reservoir and recirculated through the } \\
\text { slurry loop for } 30 \text { minutes. The acid was circulated back and forth across the } \\
\text { filter element by filling and discharging the backpulse chamber with the flow } \\
\text { meters isolated. The nitric acid solution was drained from the system, and the } \\
\text { system was rinsed with } 1 \mathrm{~L} \text { of DI water. Finally, two } 2 \mathrm{~L} \text { rinses with } \\
0.01 \mathrm{M} \mathrm{NaOH} \text { solution were performed. }\end{array}$ & N/A \\
\hline 5 & $\begin{array}{l}\text { A second } \mathrm{SrCO}_{3}(\mathrm{Test} \# 2) \text { was conducted using the same operating conditions } \\
\text { and volumes as in Step 3. After the test, the } \mathrm{SrCO}_{3} \text { was drained from the } \\
\text { system and the system rinsed with 2-L of } 0.01 \mathrm{M} \mathrm{NaOH} \text { solution. }\end{array}$ & $\begin{array}{l}\text { AV: } 13 \mathrm{ft} / \mathrm{s} \\
\text { TMP: } 40 \mathrm{psid} \\
\text { Duration: } 4 \text { hours }\end{array}$ \\
\hline 6 & $\begin{array}{l}\text { A second cleaning with nitric acid was performed to dissolve any residual } \\
\mathrm{SrCO}_{3} \text { before cleaning with oxalic acid. Acid cleaning was done as described in } \\
\text { Step } 3 \text { but for } 10 \text { minutes only. The nitric acid solution was drained from the } \\
\text { filter, and the filter rinsed with } 1 \mathrm{~L} \text { of DI water. }\end{array}$ & N/A \\
\hline 7 & $\begin{array}{l}\text { The filter system was cleaned with oxalic acid. Approximately } 2 \mathrm{~L} \text { of } 0.5 \mathrm{M} \\
\text { oxalic acid solution was added to the slurry reservoir and recirculated through } \\
\text { the slurry loop for } 30 \text { minutes. The acid was circulated back and forth across } \\
\text { the filter element by filling and discharging the backpulse chamber with the } \\
\text { flow meters isolated. The oxalic acid solution was drained, and the system was } \\
\text { then rinsed with } 1 \mathrm{~L} \text { of DI water followed by } 2 \mathrm{~L} \text { of } 0.01 \mathrm{M} \mathrm{NaOH} \text { solution. }\end{array}$ & $\mathrm{N} / \mathrm{A}$ \\
\hline 8 & $\begin{array}{l}\text { A third } \mathrm{SrCO}_{3}(\mathrm{Test} \# 3) \text { was conducted using the same operating conditions and } \\
\text { volumes as in Steps } 3 \text { and } 5 \text {. After the test, the } \mathrm{SrCO}_{3} \text { was drained from the } \\
\text { system and the system rinsed with } 2 \mathrm{~L} \text { of } 0.01 \mathrm{M} \mathrm{NaOH} \text { solution. }\end{array}$ & $\begin{array}{l}\text { AV: } 13 \mathrm{ft} / \mathrm{s} \\
\text { TMP: } 40 \mathrm{psid} \\
\text { Duration: } 4 \text { hours }\end{array}$ \\
\hline 9 & $\begin{array}{l}\text { A third and final nitric acid clean was performed to dissolve any residual } \mathrm{SrCO}_{3} \\
\text { before final CWF testing. Acid cleaning was done as described in Steps } 3 \text { and } 6 \\
\text { but for } 20 \text { minutes only. The nitric acid was drained from the system, and the } \\
\text { system was rinsed with } 1 \mathrm{~L} \text { of DI water followed by } 2 \mathrm{~L} \text { of } 0.01 \mathrm{M} \mathrm{NaOH} \\
\text { solution. }\end{array}$ & N/A \\
\hline 10 & $\begin{array}{l}\text { A final CWF test was performed. The system was loaded with } 2.5 \mathrm{~L} \text { of } \\
0.01 \mathrm{M} \mathrm{NaOH} \text { solution and a } 2 \text {-hour CWF test was performed. After CWF } \\
\text { measurements were complete, the CUF was drained and laid-up with } \\
0.01 \mathrm{M} \mathrm{NaOH} \text { solution. }\end{array}$ & $\begin{array}{l}\text { AV: } 11 \mathrm{ft} / \mathrm{s} \\
\text { TMP: } 10 \mathrm{psid} \\
\text { Duration: } 2 \text { hours }\end{array}$ \\
\hline
\end{tabular}




\subsection{Model Validation and Updating}

In addition to performing the Series 1 long-term fouling tests, it was desirable to develop a model that predicted filter performance and gave insight into the relevant physical mechanisms. The state of the art in cross-flow ultrafiltration is discussed in detail in Daniel et al. (2010a). The approach in this work is to build a model framework starting from the PNNL model reported in Daniel et al. (2010b). It was also anticipated that other theoretical approaches discussed in the survey of literature performed in Daniel et al. (2010a) would assist in building an understanding of cross-flow filtration behavior. This led to the consideration of several different models.

The models considered in this report all had at least one adjustable parameter and thus required iterative solution techniques. Given the volume of data, MATLAB ${ }^{\circledR}$ (MathWorks, Inc., Natick, MA) was chosen as the computational software used to perform the modeling. For models with coupled differential equations, a Levenberg-Marquardt algorithm (Levenberg [1944]; Marquardt [1963]) was used to minimize the sum of the squared error. Note that the Levenberg-Marquardt algorithm does not find a global minimum, only a local one. To verify a global minimum was found, the starting values for a model's adjustable parameters were varied to confirm convergence to the same answer. Other models with simpler functionality were fit using non-linear least squares search algorithm called the trust region method (see, for example, Celis et al. [1985]). The trust region method, like the Levenberg-Marquardt algorithm, uses a gradient-based approach to convergence. Both of these numerical techniques were chosen because they converge quickly and efficiently. 


\subsection{Testing Equipment}

This section provides a high-level summary of the testing equipment used to perform the experimental work summarized in this report. The equipment can be separated into two categories: 1) filtration testing equipment and 2) slurry characterization equipment. The filtration equipment used is a laboratory-scale filtration testing loop that is instrumented to monitor changes in system flow rates and pressures and how operational parameters impact filter performance. The slurry characterization equipment is a group of analytical equipment used to characterize test slurries before and after filtration to quantity how slurry physical properties impact filtration.

\subsection{Filtration Testing Equipment}

The testing apparatus was a bench-top system mounted on a skid that allowed up to $25 \mathrm{~L}$ of a simulant waste solution to be circulated through a tubular filter that can measure filter feed flow rates, filtrate flow rates, system pressures, and temperatures simultaneously. The testing apparatus used a heat exchanger on the main flow loop to cool the feed solution during filtration operations and had a heater on the main holding tank to perform leaching at elevated temperatures.

\subsubsection{Cross-flow Filter}

The cross-flow filters tested were supplied from the Mott Corporation (Farmington, CT) using the same specifications for the filters being purchased for the WTP PTF. The filters were 316 stainless steel symmetric elements from Mott Corporation with a Mott Grade of 0.1. To date, 2-foot-long and 8 -foot-long filter elements have been employed on this system. However, all the testing performed in this report used a 2-foot filter element. The dimensions of the 2-foot-long filter element are shown in Figure 4.1. A scanning electron microscopy (SEM) image of the CUF cross-section is shown in Figure 4.2. This image illustrates both pore size and tortuosity of the filter media, which allow it to retain submicron particles with high efficiency. Analysis of manufacturer data, including isopropanol bubble point and filter flux data and SEM images (Rubow and Jha 1999), indicates that the Mott grade 0.1 sintered stainless steel filter elements have an effective hydrodynamic pore diameter in the range of 1 to $3 \mu \mathrm{m}$.

The filter elements were received already installed in a shell-and-tube configuration with an outer tube surrounding the filter element to capture the filtrate while the inlet and the outlet of the filter (which extend past the shell and provide access to the inside diameter of the filter) were welded to steel tubing of a matching outer/inner diameter. The shell side had two 3/8-inch stainless steel tubes exiting from the filter assembly, one in the center to collect filtrate from the filter, and the other near the inlet of the filter to function as a drain. Pressure ports ( $1 / 4-$ inch stainless steel tubing) were installed on the inlet and outlet connections to the assembly to measure the pressure inside the filter. O-ring face seal (Swagelok VCOß) fittings purchased from the Swagelok Company (Solon, $\mathrm{OH}$ ) were also placed on the inlet and outlet filter feed tube connections for easy installation to the filtration/leaching skid. Figure 4.3 and Figure 4.4 show the CUF assembly.

\footnotetext{
${ }^{4}$ Owsiany M. 2007. "Mott HyPulse ${ }^{\circledR}$ LSX Data Summary - 24590-MOTT-FDP02.” Mott Corporation,
} Farmington, Connecticut. 

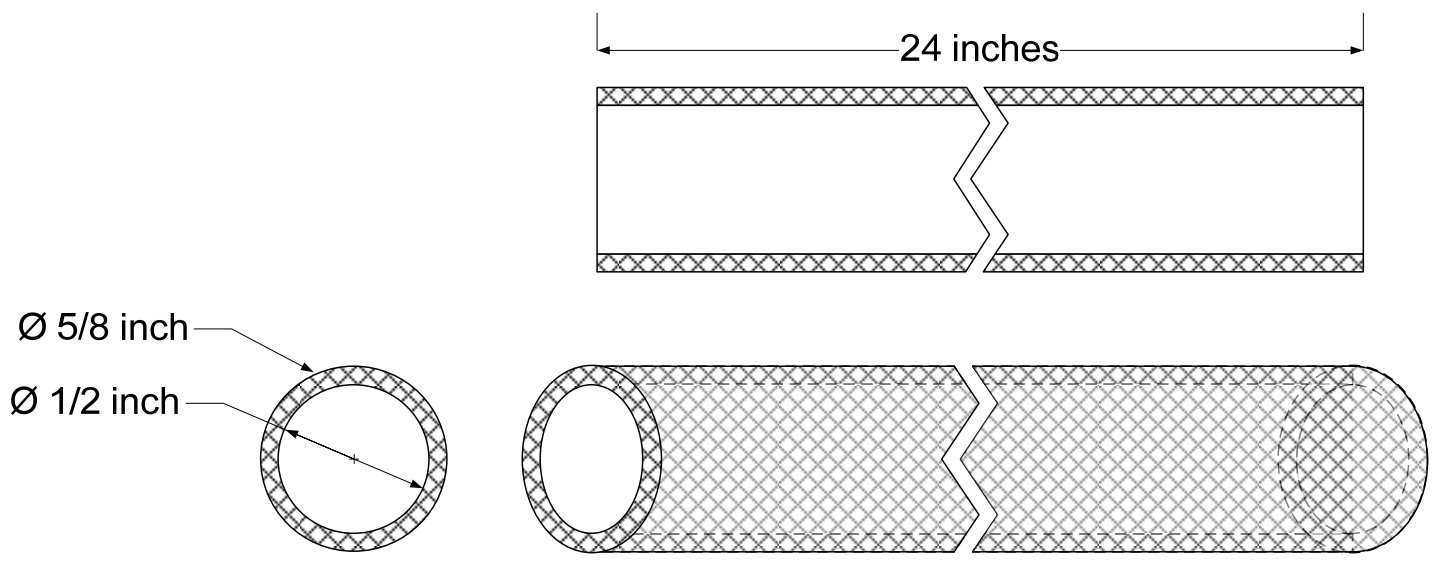

Figure 4.1. Cell Unit Filter Element

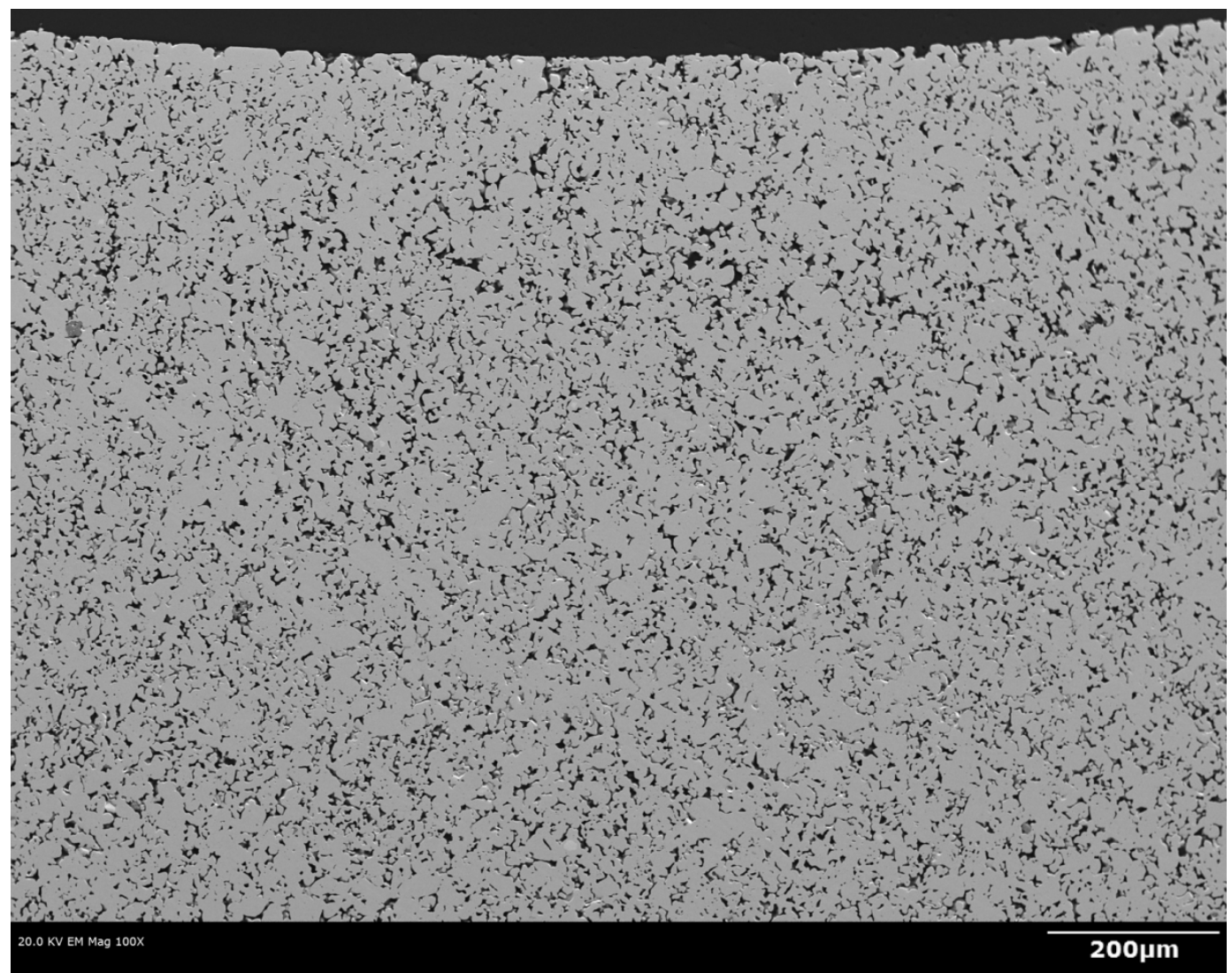

Figure 4.2. SEM Image of CUF Filter Element Cross-Section 


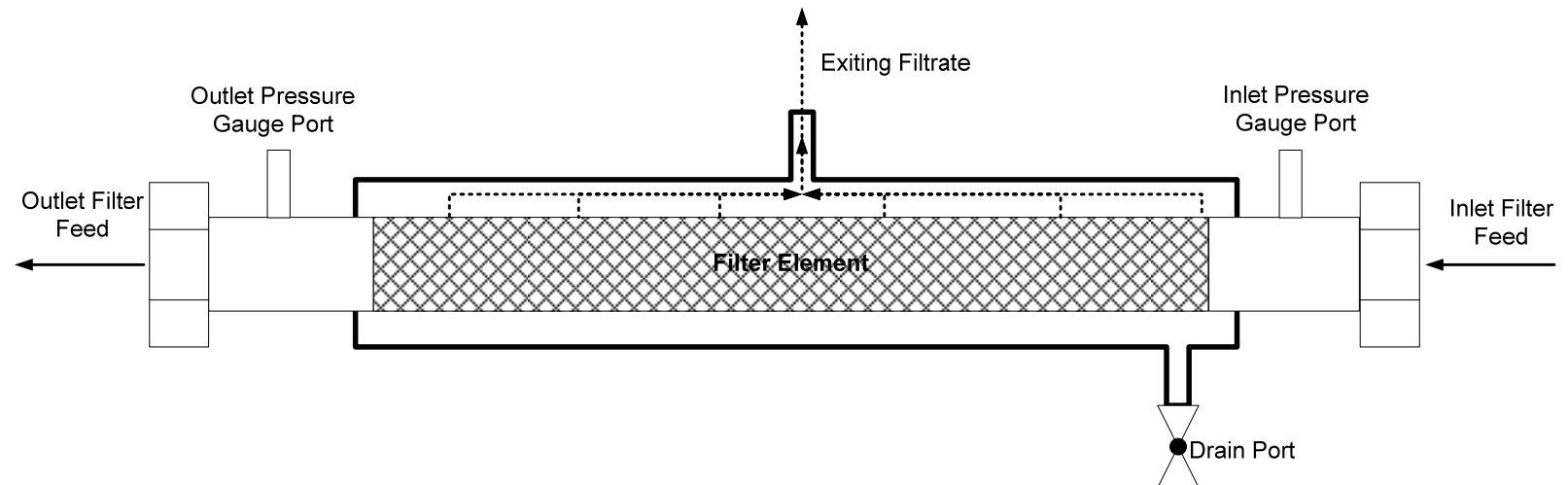

Figure 4.3. Cell Unit Filter Assembly Sketch (not to scale)

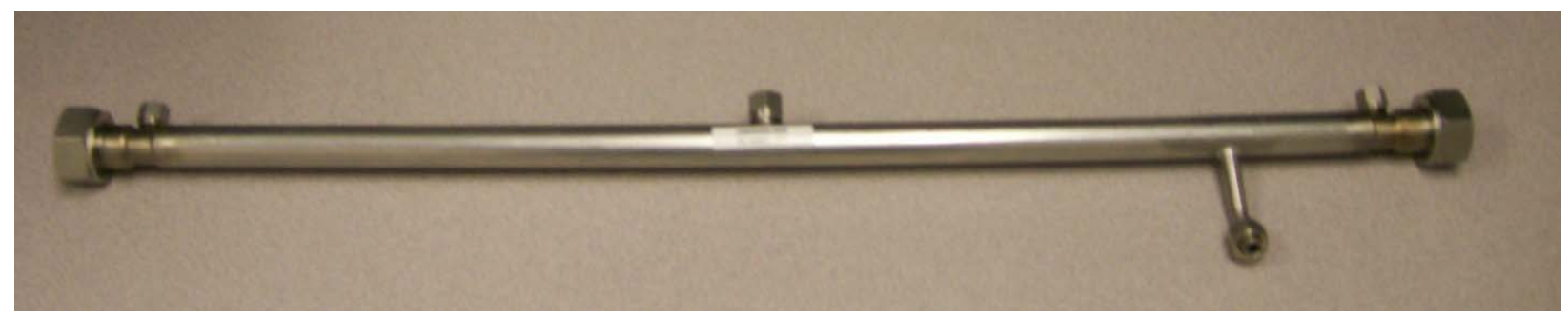

Figure 4.4. The Cell Unit Filter Assembly

It should be noted that the particular filter element selected for use in Series 1 testing has been used in previous simulant leaching and filtration tests, notably Russell et al. (2009); Daniel et al. (2009a); and Billing et al. (2009). As part of these studies, the filter element has been subjected to dozens of simulant fouling tests followed by chemical cleaning operations. The last significant filtration test performed with this filter element before Series 1 testing was a dewatering test with leached, washed PEP simulant solids for integrated PEP testing (Billing et al. 2009; Kurath et al. 2009). After this test, the system was flushed with $0.01 \mathrm{M} \mathrm{NaOH}$ solution and laid up dry for long-term storage. To prepare for Series 1 testing, the filter element (and filtration system) was fully re-wetted and vigorously cleaned with $0.01 \mathrm{M} \mathrm{NaOH}$, Alconox detergent, and oxalic acid.

At the end of Series 1 testing, this filter element was removed from the CUF for destructive testing, including optical microscope and SEM imaging. Destructive analysis of the filter was necessitated, in part, by observations of irreversible filter fouling in actual waste testing (Lumetta et al. 2009; Edwards et al. 2009; Fiskum et al. 2009; Shimskey et al. 2009a; and Shimskey et al. 2009b), in integrated PEP testing (Daniel et al. 2009b), and in the current testing (see Section 5.2). The rationale for destructive testing shall be provided in the conclusions to this report. Results of destructive analysis testing will be reported in the final report for FY11 test activities. As a result of destructive testing, a new filter element was installed on the CUF to support Series 2 testing (and accompanying Series 3 cleaning tests for Series 2 and onward). This filter element comes from the same manufacturer's lot and should have the same filtration properties as the previous filter. 


\subsubsection{Cross-flow Filtration Testing System}

The filter described in the section above was installed in a bench-top testing apparatus that circulated the test filtration simulants through the inside of the filter and diverted the filter permeate to a collection bottle or recycled it back into the slurry reservoir. Figure 4.5 shows a piping diagram of the cross-flow ultrafiltration testing system. Figure 4.6 is a photograph of the assembled testing apparatus employing a 2 -foot filter element. The 3-HP electric motor and positive displacement pump that drives the filtration slurry simulant are shown to the left in this view.

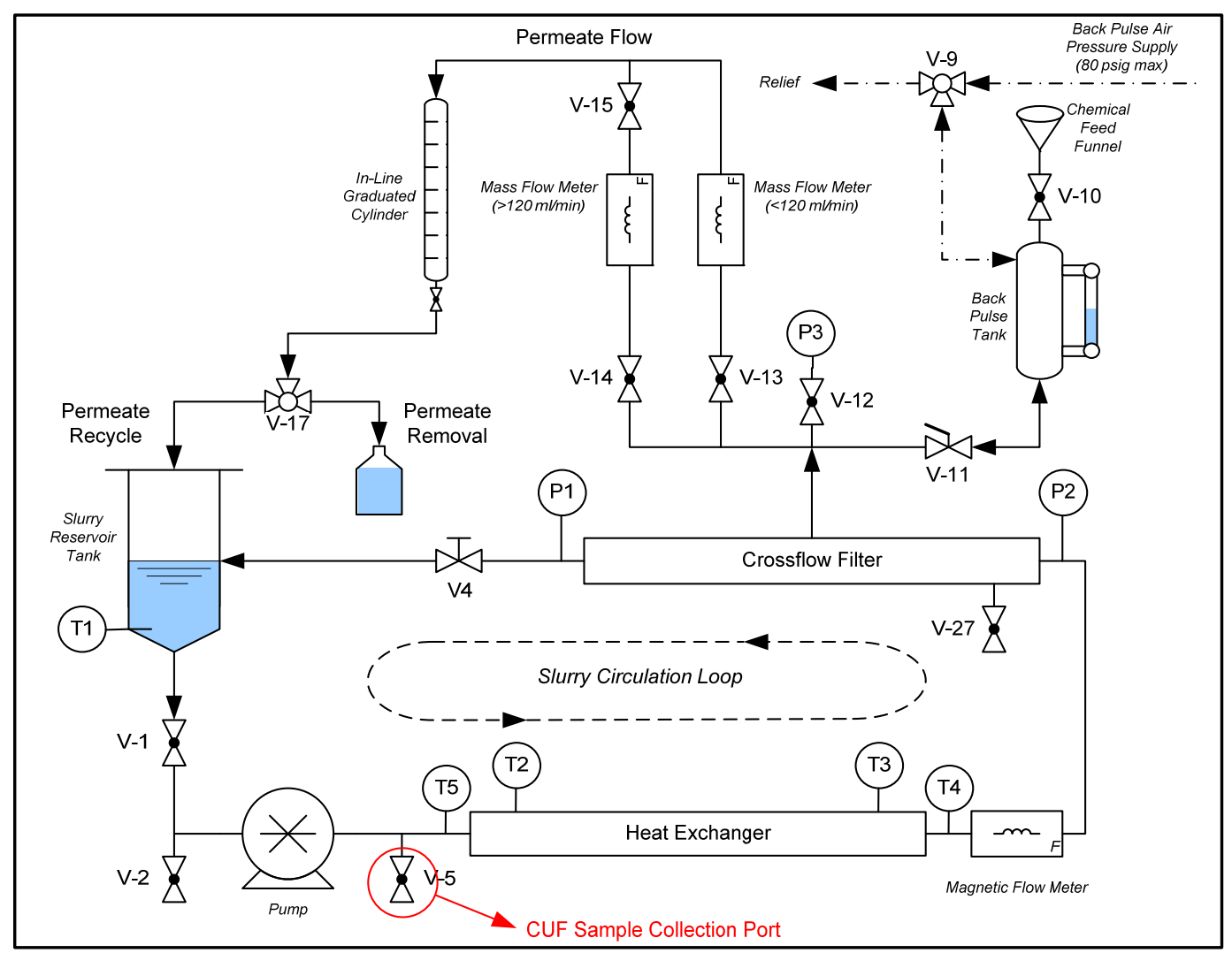

Figure 4.5. CUF Piping Diagram 


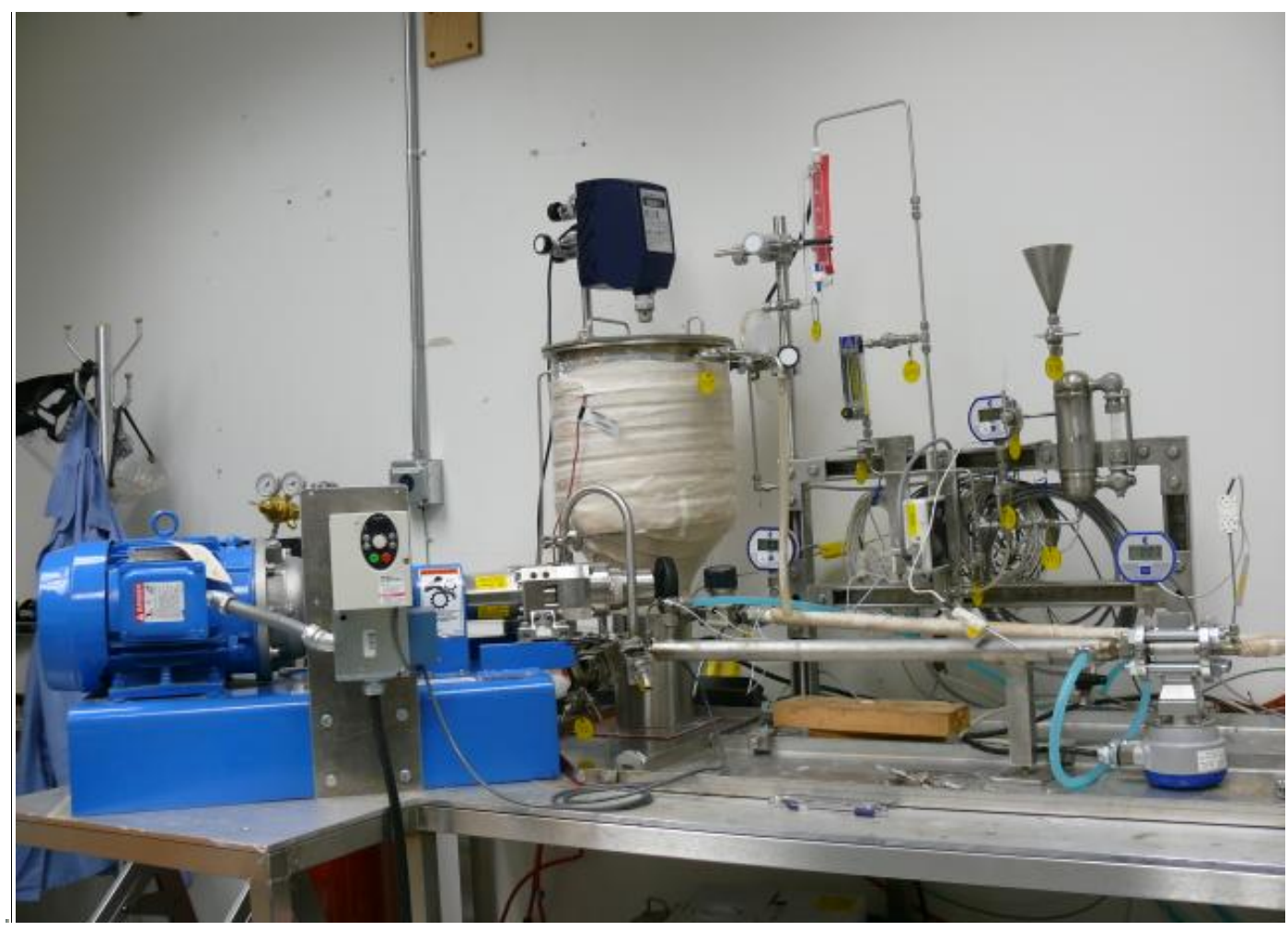

Figure 4.6. The CUF Apparatus

The CUF has four main parts:

- slurry reservoir tank

- slurry recirculation loop

- permeate flow loop

- permeate backpulse chamber.

The slurry reservoir tank was a cylindrical 304-L stainless steel tank. Two tanks could be used with the testing apparatus depending on the test to be performed, one of 10-L capacity and the other of 25-L capacity. The larger reservoir, as shown in Figure 4.6, permitted scaled leaching tests to be performed. The smaller reservoir allowed smaller volumes of simulant to be used. For all tests discussed herein (i.e., the scale-up and temperature tests described in Sections 6 and 7 of this report), the large 25-L tank was employed.

Agitation in the tank was provided from an overhead mixer using a 2-inch-diameter, 3-blade marine propeller. To allow the system to be easily drained, the bottom of the vessel was sloped at a $15^{\circ}$ angle. Baffles were also installed on the tank wall to enhance mixing and slurry homogeneity. Heat tape was installed around the walls of the tank for leaching at elevated temperatures. The heat tape was connected to a temperature controller that adjusted the electrical load to the heat tape based on a thermocouple input. A dual Type-K thermocouple was installed inside the reservoir tank (extending just below the overhead mixing impeller) to measure the temperature of the slurry inside the reservoir. One of the thermocouple elements was connected to the heat tape's temperature controller and the other was connected to a data collection system. 
The slurry recirculation loop directed slurry flow from the slurry reservoir through the filter and back into the slurry reservoir for filtration operations. The bottom of the slurry reservoir was connected to the suction side of the slurry recirculation pump - a progressive cavity pump. The discharge of the pump flowed through a single-pass shell and tube heat exchanger used to remove excess heat from the system from mechanical energy input from the mixer and pump and heat generated from frictional flow.

An exterior chiller circulated a water/anti-freeze mixture through the exterior shell of the heat exchanger to remove heat away from the circulating slurry on the tube side of the heat exchanger. The chiller controlled the chilling fluid temperature by monitoring the temperature of the slurry exiting the heat exchanger via a resistance temperature detector (RTD) installed in the discharge line.

The slurry then flowed through a magnetic flow sensor that monitored the volumetric flow of the slurry inside the slurry recirculation loop. The sensor's output was displayed on an external panel meter that generated an analog output signal monitored by a data collection system. The data from this device were used to calculate the AV inside the filter element.

The flowing slurry then entered the filter. Digital pressure gauges were installed on the inlet and outlet port of the filter, which displayed the pressure at both locations in a pounds per inch squared gauge (psig). The gauges also transmitted analog output signals monitored by the data collection system. The data from these devices were used to calculate the average pressure inside the filter and the axial pressure drop across the element.

A manual pinch valve was located at the filter's discharge. The valve was used to adjust the pressure inside the filter to drive permeate flow through the filter membrane wall. It was also connected to the slurry reservoir tank and was closed completely when the tank was isolated for leaching.

The permeate flow loop started at the center of the filter assembly where a poly-line connected the filter to a manifold of $3 / 8$-inch stainless steel piping that directed the filter permeate through a series of measurement devices. A digital pressure gauge was installed at this point to measure the pressure on the permeate side of the filter in psig. Like the other two digital gauges, this instrument transmitted an analog output signal to a data collection system. The TMP across the filter was then calculated by subtracting the pressure on the permeate side of the filter from the average pressure of the slurry inside the filter. TMP is reported in psid.

Flow from the filter was either diverted through a mass flow meter calibrated up to $120 \mathrm{~mL} / \mathrm{min}$ or a mass flow meter calibrated up to $1.2 \mathrm{~L} / \mathrm{min}$, and the flow output was transmitted to the data collection system. Both of the mass flow meters measure density of the permeate flow, but only one of the instruments $(120 \mathrm{~mL} / \mathrm{min}$ system $)$ transmits data to the data collection system. An in-line glass cylinder was installed on the discharge of both meters to take manual measurements of the permeate flow rate. Measurements were taken by closing a valve at the bottom of the cylinder and allowing permeate to fill the glass cylinder. Liquid volume in the glass cylinder was measured by volume markings on the outside. The permeate flow rate was calculated from observed changes in permeate volume in the cylinder over a measured time interval.

Permeate exited through a three-way valve connected to the slurry reservoir tank. This valve directed permeate either back to the slurry reservoir tank to be mixed with the slurry or to a sampling hose used to collect permeate into sample containers. 
The permeate backpulse chamber was located to the right of the permeate flow loop and connected to the filter at the same location of the permeate pressure gauge. The chamber was an approximately $1000-\mathrm{mL}$ steel vessel with a sight-glass to track the permeate volume inside the chamber. The vessel had three entry ports:

- $\mathrm{a}^{3} / 8$-inch line with a two-way valve on the bottom connecting the vessel to the permeate side of the filter

- $\mathrm{a}^{3} / 8$-inch line with a two-way valve connecting the top of the vessel to a funnel

- a $1 / 4$-inch line with a three-way valve connecting the top of the vessel to a compressed air line and vent line connected to the top of the slurry reservoir tank.

The bottom line was used to direct permeate flow between the chamber to the filter. The funnel on the top of the chamber was used to introduce cleaning and rinse solutions directly to the vessel. The compressed gas line was used to pressurize the fluid in the chamber with compressed gas and to vent the chamber to atmospheric pressure.

To backpulse the filter, the vessel was first vented to atmospheric pressure. Next, permeate was allowed to fill the chamber by opening the valve. Once the chamber was half full of permeate (as seen from the sight-glass), the valve was closed. The three-way valve was then positioned to allow compressed gas at 80 psig to fill the chamber and pressurize the fluid. The three-way valve was then positioned to isolate the now pressurized chamber. The slurry pressure inside the filter was then dropped below 20 psig. The valve at the bottom of the tank was opened, allowing the pressurized permeate inside the chamber to flow backwards through the filter element. The valve was closed when the permeate level was below the visible portion of the sight glass. After the backpulse was completed, the three-way valve was positioned to vent the chamber back to atmospheric pressure.

\subsubsection{Instrumentation and Data-Acquisition System}

Most of the sensors on the testing apparatus transmit analog data to an external data-acquisition collection system (DACS) from the National Instruments Corporation (Austin, TX). This system relayed the analog data to a LabView data collection program operating on a computer desktop system using Windows XPтM, Service Pack 2. The software program scales the analog data, simultaneously records the data electronically, and displays it on the computer monitor. The program was verified and all reportable data are measured on calibrated instrumentation including the external DACS board.

Figure 4.7 shows a diagram of the electronic sensors attached to the DACS, and Figure 4.8 displays the screen windows from the data collection program. 


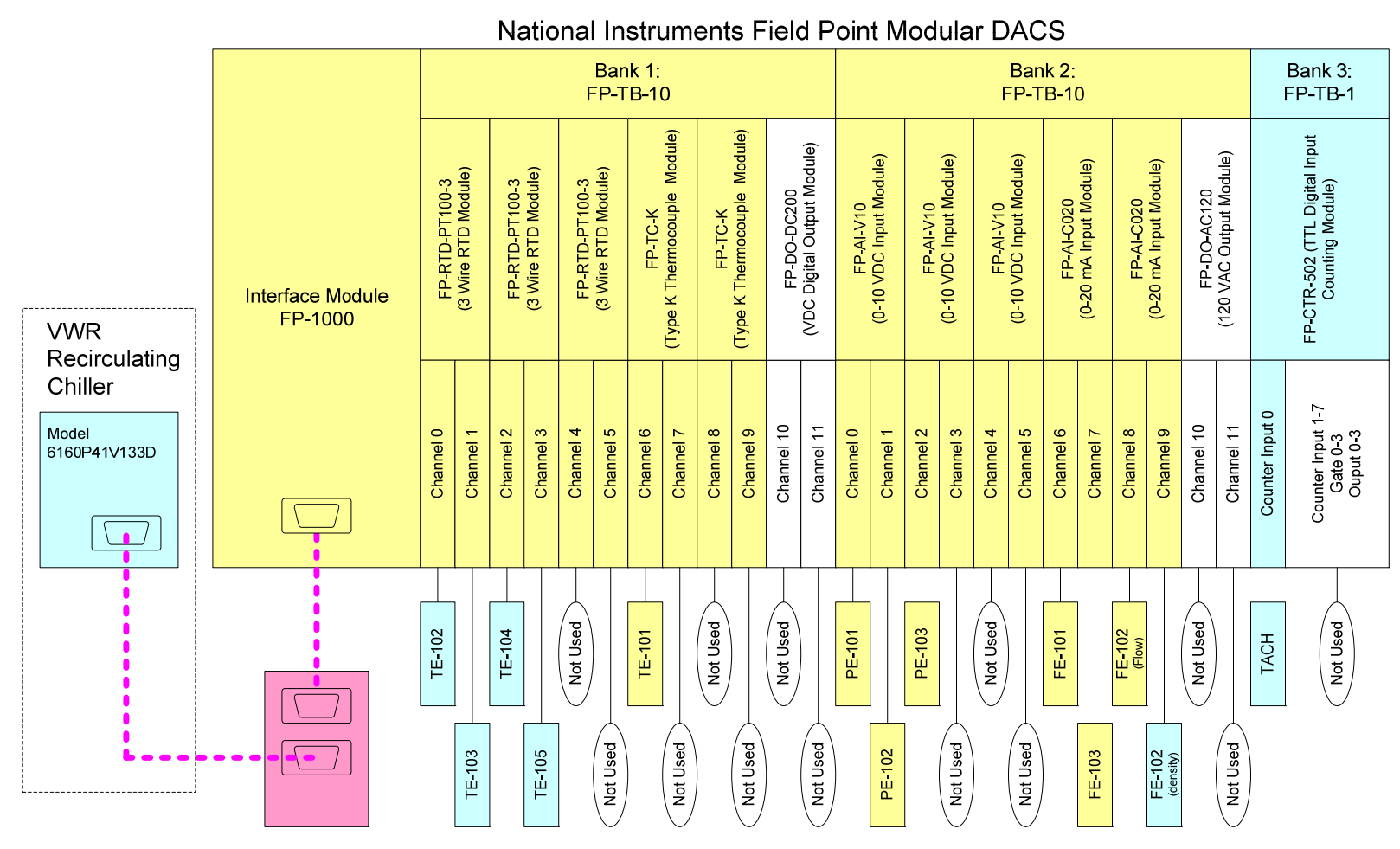

\begin{tabular}{|lll|}
\hline Windows XP PC & M\&TE Data \\
$\cdots$ RS-232 Communication & $\square$ Indication Only
\end{tabular}

Figure 4.7. Diagram of DACS System 


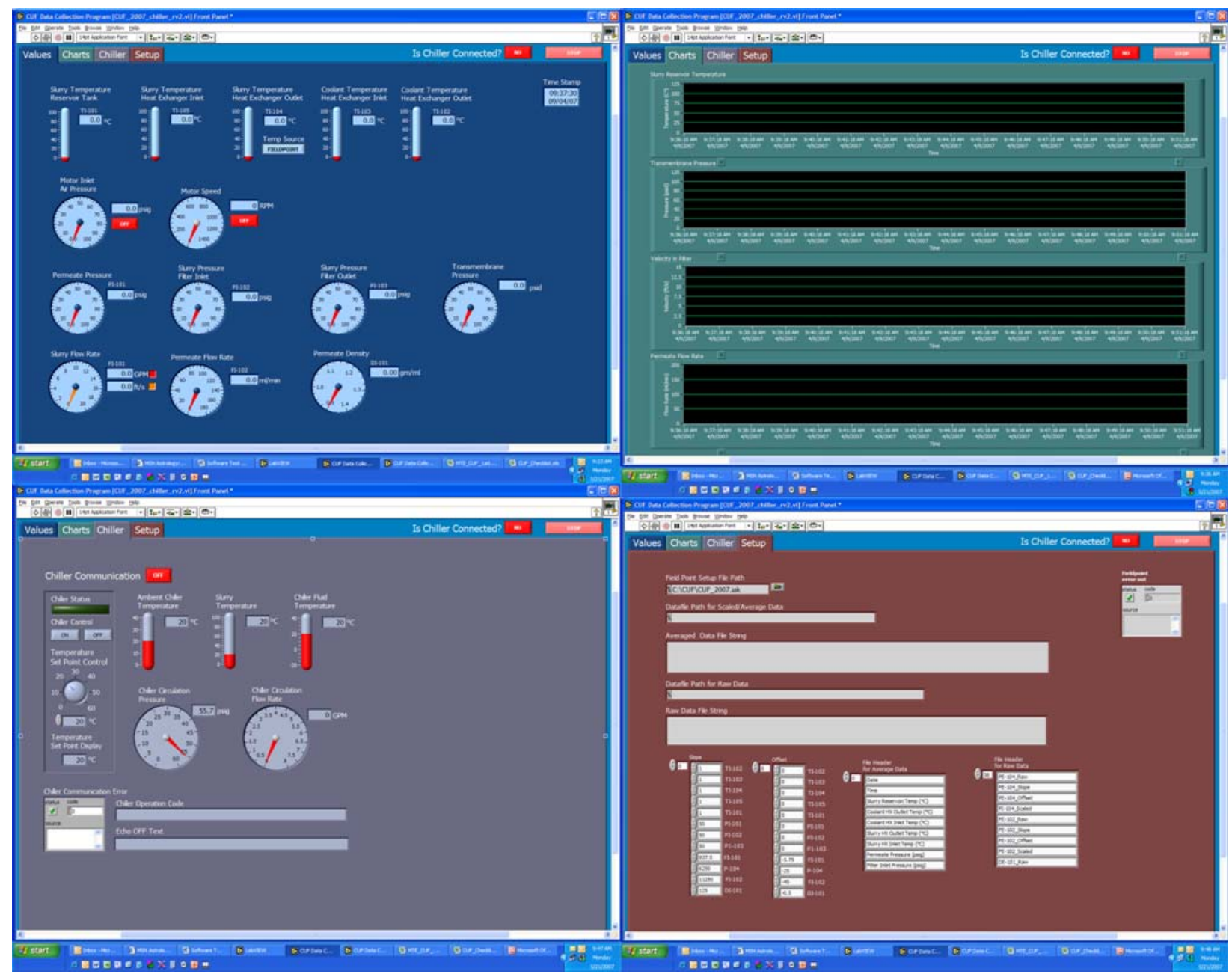

Figure 4.8. Digital Images of DACS Display Windows

\subsubsection{Operations of Cell Unit Filter and Sampling}

The CUF was developed to operate in several different modes to simulate filtration and leaching processes of the WTP pretreatment system. Filtration operation can occur in a recycling or dewatering mode. During recycling operations, permeate is returned to the slurry reservoir tank. The CUF was only run in recycle mode during the tests presented in this report. By returning permeate back into the slurry, the UDS concentration in the slurry was maintained at a steady-state condition. The CUF was operated in this mode to understand how the effects of time, pressure, and AV impact filtration of slurry while maintaining the physical properties of the slurry. The CUF can also be operated in a dewatering mode (not used in the tests described in this report) to understand how the slurry's rheological and filtration properties changed as its UDS concentration changed. During dewatering operations, permeate from the filter was diverted to a collection vessel, operating the system at a constant TMP and axial flow rate, thus altering the UDS concentration of the slurry.

Samples were collected throughout testing to measure the physical and chemical properties of the waste slurry or permeate. Slurry samples were collected from two separate locations on the system. When possible, slurry samples were collected using the drain valve on the pump discharge while the 
pump was running. Permeate samples were collected during dewatering operations directly from the dewatering sample hose.

\subsection{Simulant Characterization Equipment}

Simulant characterization involved measurement of several physical properties relevant to filtration. These properties included undissolved solids concentration, dissolved solids concentration, permeate viscosity, slurry rheology, particle size analysis, and isoelectric point (IEP) measurement.

\subsubsection{Solid Concentration Analysis}

Analysis of PEP simulant solid concentration included determination of the total solids content of the slurry (which includes both dissolved and undissolved solids), slurry undissolved solids, and supernate dissolved solids. These analyses employed an HR83 halogen moisture analyzer from Mettler Toledo. Total weight percent solids, $x_{T}$, is defined as the mass of dried solids per mass initial wet mass of sample before drying. Total solids were determined by drying an aliquot of PEP simulant slurry in the HR83 moisture analyzer. Supernate dissolved solids, $x_{D S}$, is defined as the mass fraction of solids dissolved in a supernate sample. It was determined by drying an aliquot of PEP supernate in the HR 83 moisture analyzer. Supernate samples were obtained by first centrifuging a sub-aliquot of PEP slurry to settle the slurry solids and then carefully decanting the supernate off the settled solids. Undissolved solids concentration, $x_{U D S}$, is defined as the mass fraction of undissolved solids in a slurry sample. It was calculated from values of $x_{T}$ and $x_{D S}$ using the following formula:

$$
x_{U D S}=\frac{x_{T}-x_{D S}}{1-x_{D S}}
$$

All solids content analyses performed in the HR83 moisture analyzer involved heating the sample to $95^{\circ} \mathrm{C}$ for 30 minutes to drive off bulk moisture without boiling. Then, the sample is heated to $105^{\circ} \mathrm{C}$ to drive off the remaining moisture until the sample reached a weight stability criterion 5 in the Mettler Toledo user interface settings. Weight percent solids is then determined by dividing the dried solids weight by the initial sample mass.

\subsubsection{Slurry and Supernate Rheological Measurements}

Aliquots of PEP slurry were taken before and after CUF testing of the slurry for the purpose of measuring slurry rheology and supernate viscosity. PEP simulant supernate samples were obtained by first centrifuging a sub-aliquot of PEP slurry to settle the slurry solids and then carefully decanting the supernate off the settled solids. Slurry rheology and supernate viscosity were both measured using a Haake RS600 rheometer (now sold by Thermo Fischer Scientific, Waltham, 02454 USA). A Z41 concentric cylinder measuring system was employed for testing. Rheometer control and data acquisition are accomplished through remote computer connection using the RheoWin Pro Job Manager Software, Version 2.96 .

Flow curve measurements (i.e., material stress response versus applied shear rate) at a control temperature of $25^{\circ} \mathrm{C}$ were performed for each slurry and supernate sample collected. Each flow curve was measured over an 11-minute period. During the first 5 minutes, the shear rate was gradually increased from zero up to a pre-defined maximum shear rate (typically between 200 and $1000 \mathrm{~s}-1$ ). Next, 
the shear rate was held constant at the predefined maximum shear rate for 1 minute. For the final 5 minutes, the shear rate was gradually reduced back to zero. During this time, the resisting torque and rotational rate were continuously monitored and recorded. After measurement, the recorded flow curve data were analyzed with linear least-squares regression analysis to determine best fit values for supernate viscosity and slurry rheology. Slurry rheology was fit using the Bingham Plastic model, and best fit values for the Bingham yield stress and consistency were derived.

\subsubsection{Slurry and Supernate Density Measurements}

Aliquots of PEP slurry were taken before and after CUF testing of the slurry for the purpose of measuring slurry and supernate density. PEP simulant supernate samples were obtained by first centrifuging a sub-aliquot of PEP slurry to settle the slurry solids and then carefully decanting the supernate off the settled solids. Density measurements were made using glass pycnometers. Each pycnometer had a standardized volume. For each density measurement, an aliquot of PEP slurry or supernate was loaded into a pre-weighed pycnometer to the marked level. The total mass of the filled pycnometer was weighed using a 3-place analytical balance. Next, the mass of fluid added to the pycnometer was determined by the difference of the total filled mass and the pycnometer tare. Density was then determined by dividing fluid mass by the standardized pycnometer volume.

\subsubsection{Particle Size Analysis}

Particle size characterization was accomplished using a Mastersizer 2000 (Malvern Instruments, Inc., Southborough, MA 01772 USA) with a Hydro S wet dispersion accessory. The Mastersizer has a nominal size measurement range of 0.02-2000 $\mu \mathrm{m}$. The actual range is dependent on the accessory used as well as the properties of the solids being analyzed. When coupled with the Hydro $\mu \mathrm{P}$ wet dispersion accessory, the nominal measuring range is reduced to $0.02-600 \mu \mathrm{m}$ (also dependent on material density). Although particle sizes above $600 \mu \mathrm{m}$ can be observed with the Hydro G, reliable determination of their volume contribution is a challenge for the instrument. However, the PEP simulant slurry is not anticipated to contain particles larger than $600 \mu \mathrm{m}$, and as such, issues associated with suspending and detecting large/dense particles were not anticipated. Measurement data were recorded and analyzed using Mastersizer 2000 software, Version 5.6 (Malvern Instruments, Ltd. Copyright (C) 1998-2009). Table 4.1 provides a summary of basic information regarding the analyzer and accessory used.

Table 4.1. Summary of Malvern Mastersizer 2000 Instrument Information

\begin{tabular}{ll}
\hline \multicolumn{1}{c}{ Analyzer } & \multicolumn{1}{c}{ Mastersizer 2000 } \\
\hline Measurement principle: & Laser Diffraction (Mie Scattering) \\
Analyzer Accessory: & Hydro G \\
Measurement Range: & $0.02-600 \mu \mathrm{m}$ nominal \\
Type: & Flow cell system with continuously variable and \\
& independent pump and ultrasound. \\
Pump Speed/Stirrer Range: & $0-3500$ rpm (variable) \\
Ultrasound Power & $0-20 \mathrm{~W}$ (variable) \\
Software Version/date & $5.6 / 1998-2009$ \\
\hline
\end{tabular}




\subsubsection{Isoelectric Point (IEP) Measurements}

The IEP of individual PEP simulant components employed in Series 2 testing were measured at DOE's William R. Wiley Environmental Molecular Sciences Laboratory (EMSL) user-facility at PNNL under user proposals 41490 and 41492. Measurements were made using a Brookhaven ZetaPALS zeta potential analyzer from Brookhaven Instruments Corporation (Holtsville, NY 11742) equipped with an automated dispersion titrator. Tests suspensions were prepared at $0.1-\mathrm{vol} \%$ dispersions in $0.05 \mathrm{M}$ $\mathrm{NaNO}_{3}$. Measurements were made by first loading a sub-aliquot of the test dispersion into the instrument, diluting the dispersion with $0.05 \mathrm{M}$ NaNO3 solution until an acceptable testing concentration was achieved. Once the sample had been loaded and adjusted to an acceptable concentration, $\mathrm{pH}$ was adjusted to a desired setpoint by titrating the dispersion with acid or base solution. The zeta potential of the dispersion was then measured. The process of $\mathrm{pH}$ adjustment was repeated several times, and several zeta potential measurements of the dispersion over $\mathrm{pH}$ of 3 to 10 were made. Titrations used either $0.01 \mathrm{M}$ $\mathrm{NaOH}$ in $0.04 \mathrm{M} \mathrm{NaNO}_{3}$ solution or $0.01 \mathrm{M} \mathrm{HNO}_{3}$ in $0.04 \mathrm{M} \mathrm{NaNO}_{3}$ solution. Measurements of zeta potential over a range of $\mathrm{pH}$ produce a zeta potential curve as a function of $\mathrm{pH}$ for the particle system analyzed. This curve is typically positive at low $\mathrm{pH}$ and negative at high $\mathrm{pH}$. The isoelectric point corresponds to the $\mathrm{pH}$ at which the zeta potential curve crosses zero, and is determined from the measurement data by interpolation.

\subsection{Data Analysis}

The CUF test system includes instrumentation for measuring 1) filter AV, 2) filter TMP, and 3) rate of permeate production and density. Data from CUF instrumentation are handled by the CUF data acquisition (described in Section 4.1.3) and are posted to a plain text file. The CUF records data at $0.4 \mathrm{~Hz}$ and also generates data processed and averaged over 1-minute intervals. Raw $(0.4 \mathrm{~Hz})$ and 1 -minute average data are posted to two separate plain text files. The output data parameters generated by the CUF DACS for the 1-minute data results are listed in Table 4.2. Raw data files include all the information necessary to recreate the averaged data.

Table 4.2. CUF Filtration System Measurement Instrumentation

\begin{tabular}{ll}
\hline \multicolumn{1}{c}{ Parameter } & \multicolumn{1}{c}{ Units } \\
\hline Slurry Reservoir Temperature & ${ }^{\circ} \mathrm{C}$ \\
Permeate Pressure & $\mathrm{psig}$ \\
Filter Inlet Pressure & $\mathrm{psig}$ \\
Filter Outlet Pressure & $\mathrm{psig}$ \\
Filter TMP & $\mathrm{psid}$ \\
Volumetric Slurry Flow & $\mathrm{GPM}$ \\
Filter AV & $\mathrm{ft} / \mathrm{s}$ \\
Permeate Flow & $\mathrm{mL} / \mathrm{min}$ \\
\hline Permeate Density & $\mathrm{g} / \mathrm{mL}$ \\
\hline
\end{tabular}


The current studies used only the 1-minute averaged data for filter flux analysis and comparison. However, under certain operating conditions (such as TMPs $\sim 60 \mathrm{psid}$ and $\mathrm{AV} \sim 17 \mathrm{ft} / \mathrm{s}$ ), the raw and averaged data contained a significant number of outliers, especially in measurements of permeate flow rate (see Figure 4.9). At steady operating conditions (i.e., constant AV and TMP), permeate flux is expected to be well behaved, such that a steady continuous decline in permeate rate occurs or a steady permeate rate is held. Figure 4.9 includes both start-up ( 0 to 2 hours elapsed time) and steady operating conditions of $40 \mathrm{psid}$ and $17 \mathrm{ft} / \mathrm{s}$ (greater than 2 hours elapsed time). Even during periods of steady operating conditions, periodic spikes (either a brief jump or drop) are observed in the averaged permeate rate. Examination of the $0.4-\mathrm{Hz}$ data file indicates that these spikes typically correspond to a single outlier in each set of 24 averaged values (which correspond to 1-minute worth of data points). While the exact cause of data spiking is unknown, vibration, electrical interference, and bubble formation in the low pressure permeate lines are all suspected causes. As they do not occur at lower TMP/AV setpoints, and as their occurrence cannot be associated with any observed changes in system operating conditions, it is currently believed that these outliers do not represent real filtration performance data.

Inclusion of these outliers has a significant impact on the ability to analyze and fit the data to existing filtration fouling models (as will be done in Section 8). Because these outliers are considered as unrepresentative of the actual filtration behavior, the raw $0.4-\mathrm{Hz}$ data was reanalyzed and reaveraged to exclude any outliers from the data set. Exclusion was based on Dixon's Q-Test using an 80\% confidence limit (Rorabacher 1991). For each parameter listed in Table 4.2, the Q-Test was applied to each subset of 24 points used to generate the averaged parameter value until 1) the subset of selected data points passed the Q-Test or 2) a maximum of 3 data points had been rejected from that subset of 24 points. An example of the impact of this Q-Test on the recalculated 1-minute average data is shown in Figure 4.9. Application of the Q-Test removes most (but not all) of the outliers, while preserving process step changes associated with changing conditions (e.g., start-up activities during 0 to 2 hours elapsed time).

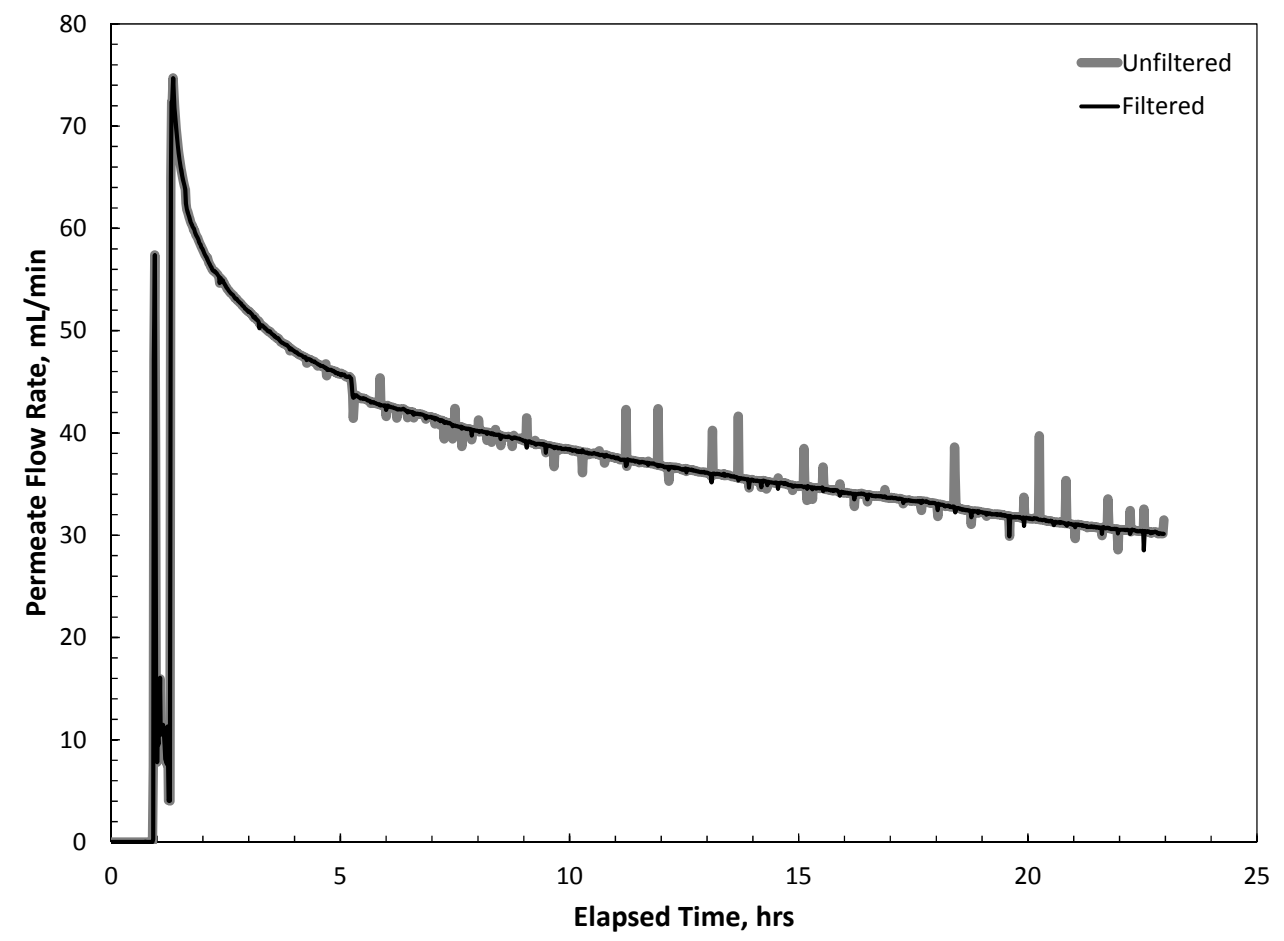

Figure 4.9. CUF 1-Minute Averaged Data Before and After Filtering Using Dixon's Q-Test 
After data filtering and reaveraging, the CUF data was further processed to calculate filter flux and to include temperature and TMP corrections to the filter flux value. First, permeate rate provided by the DAS, $Q_{p}$, was corrected for any deviations in the slurry reservoir temperature from the target operating temperature of $25^{\circ} \mathrm{C}$ using:

$$
Q_{c}=Q_{p} \exp \left[2500\left(\frac{1}{T_{a c t}}-\frac{1}{T_{\text {ref }}}\right)\right]
$$

Here, $Q_{c}$ is the corrected permeate production rate and $T_{a c t}$ is the actual temperature of the slurry reservoir (in Kelvin) and $T_{r e f}$ is the reference temperature for correction $(298.15 \mathrm{~K})$. It should be noted that value of 2500 in the exponent is taken from historical filtration studies (Geeting et al. 2003). A more complete examination of temperature corrections for permeate flux is given in Daniel et al. (2009a). After the corrected permeate rate is calculated, the filter flux may be calculated using:

$$
J=\frac{Q_{c}}{A}
$$

where $J$ is the filter flux, $Q_{c}$ is the temperature- and TMP-corrected volumetric permeate flow rate, and $A$ is the filtration surface area. The filter area is calculated using:

$$
A=\pi D_{\text {inner }} L
$$

where $D_{\text {inner }}$ is the filter element inner diameter ( 0.5 inches), and $L$ the filter element length ( 2 feet). Finally, the filter flux was corrected for deviations in actual TMP, $\Delta P_{\text {act }}$, from the TMP setpoint, $\Delta P_{\text {set }}$, using:

$$
J_{c}=\left[\frac{\Delta P_{s e t}}{\Delta P_{a c t}}\right] \cdot J
$$

Here, $J_{c}$ is the TMP-corrected flux. It should be noted that Equation 4.5 cannot strictly be applied when a compressible cake exists. Filter cakes formed by inorganic solid slurries are generally considered irreversibly compressible such that they compress when TMP is increased but do not expand when TMP is lowered (McCarthy et al. 2002). Given this understanding, it should be acceptable to correct flux when the actual TMP falls above the target TMP, but not when the actual TMP falls below the target value. The appropriateness of using Equation 4.5 to correct for small negative deviations in TMP and for oscillations of actual TMP around the target value has not been quantified. For the purpose of the current analysis, Equation 4.5 has been used to correct all TMP deviations. Because efforts were made to ensure that the actual TMP was close to the TMP target during testing, corrections are only made for small TMP deviations ( $\sim 1$ psid) and for TMP oscillations. One exception is made for analysis of clean water flux data. In general, clean water flux data are measured at a TMP of 10 psid. For the purpose of comparison, many of clean water flux results presented in Section 7 are scaled to a baseline TMP of 40 psid. While this scaling may be appropriate for clean fluids, it should be recognized that clean water flux data indicates the presence of trace solids that could potentially form a compressible cake layer. As a result, scale clean water (or other clean fluid) fluxes may overestimate fluxes that can be achieved for systems with trace solids. 


\subsection{Series 1 Testing Results}

In Series 1 testing, five cross-flow filtration tests were performed to assess long-term decline of filter flux with time to determine 1) the existence of a steady-state flux and 2) the value of flux at steady-state should the steady-state flux exist. Tests used as-prepared PEP simulant slurry developed for Integrated Tests A and B. In this section, the results of Series 1 testing are presented in detail. In Section 5.1, the physical properties of the PEP simulant used for the current set of tests are presented and verified against previous measurements made for the PEP simulant. In Section 5.2, the results of Series 1 testing are presented and discussed in detail. Finally, Section 5.3 gives a brief summary of important Series 1 test findings. The results presented in this section feed into the continued waste filtration modeling efforts presented in Section 8.

\subsection{Waste Simulant}

As stated in the introduction to this section, all Series 1 filtration tests employed an as-prepared PEP simulant slurry developed for Integrated Tests A and B (see Guzman-Leong et al. 2009; Geeting et al. 2009; and Kurath et al. 2009). Supporting documentation for the production and procurement of this simulant can be found in Scheele et al. (2009). The PEP test slurry was unused test material left over from PEP testing activities. It was obtained for EM31 use from the Process Development Laboratory West (PDLW), where it had been stored in a 55-gallon drum. After receipt, the slurry was inspected and processed for use in Series 1 testing. A volume of slurry sufficient for Series 1 testing was transferred from the 55-gallon drum into several 20-L carboys (for ease of handling) that were used as source containers for the five Series 1 tests outlined in Table 3.1. Both before and during use, several sub-aliquots of slurry were taken to allow measurement of physical properties including slurry and supernate rheology, density, and moisture content. The methods used to measure these physical properties are presented in Section 4.2.

Table 5.1 presents the measured physical properties for the PEP simulant slurry used in Series 1 testing. As stated in the previous paragraph, the mean value includes measurements of PEP slurry aliquots taken before, during, and after filtration tests. The implicit assumption in averaging these values is that there was no test-to-test sampling variability in each PEP slurry aliquot (i.e., that slurry sampling from both the 55-gallon drum and the smaller 20-L carboys was representative). Figure 5.1 and Table 5.2 show the averaged particle size distribution of PEP simulant before and after the 100-hour tests outlined in Section 3.1. Both distributions shown correspond to unsonicated solids and likely include both primary particles and particle agglomerates. As shown clearly by Figure 5.1, pumping of simulant reduces particle size. The likely cause of this reduction in size is disruption of particle agglomerates by shear.

For comparison of the analytical results presented in Table 5.1, Table 5.2, and Figure 5.1, the range of physical properties determined during integrated PEP testing of the same simulant is presented in Table 5.3. In general, the values compare well. The greatest difference is observed for the Bingham consistency and is likely a result of the difference in undissolved solids content between Series 1 and actual PEP testing (6.3-wt $\%$ versus $6.9-\mathrm{wt} \%$, respectively). 
Table 5.1. Physical Properties of PEP Simulant Slurry Measured During Series 1 Testing

\begin{tabular}{lccc}
\hline \multicolumn{1}{c}{ Physical Property } & Mean & $\begin{array}{c}\text { Error in } \\
\text { Mean }^{(\mathrm{a})}\end{array}$ & $\begin{array}{c}\text { Measurement } \\
\text { Variability }^{(\mathrm{b})}\end{array}$ \\
\hline Rheology: & & & \\
$\quad$ Bingham Yield Stress, Pa & 0.06 & \pm 0.01 & \pm 0.04 \\
$\quad$ Bingham Consistency, mPa s & 3.73 & \pm 0.03 & \pm 0.12 \\
$\quad$ Supernate Viscosity, mPa s & 2.80 & \pm 0.03 & \pm 0.13 \\
Density: & & & \\
$\quad$ Slurry Density, kg L & & \\
$\quad$ Supernate Density, kg L & & & \pm 0.010 \\
Solids Concentrations: & 1.283 & \pm 0.002 & \pm 0.0030 \\
$\quad$ Total Solids, wt\% & & \pm 0.0005 & \\
$\quad$ Undissolved Solids, wt\% & 31.1 & \pm 0.1 & \pm 0.9 \\
$\quad$ Supernate Dissolved Solids, wt\% & 6.3 & \pm 0.2 & \pm 1.1 \\
\hline
\end{tabular}

(a) The error in the mean is calculated as $z_{95} \sigma / \sqrt{N}$, where $z_{95}$ is $1.96(95 \%$ confidence limit multiplier), $\sigma$ is the sample standard deviation, and $N$ is the number of measurements used to compute the mean. $N$ was greater than 30 for all measurements except rheology, which had $N$ of 20 or greater.

(b) The measurement variability is computed as $z_{95} \sigma$, and represents the expected range of measurement variation for any given sample. With exception of rheology measurements, $N$ was greater than 30 for all measurements except rheology, which had $N$ of 20 or greater.

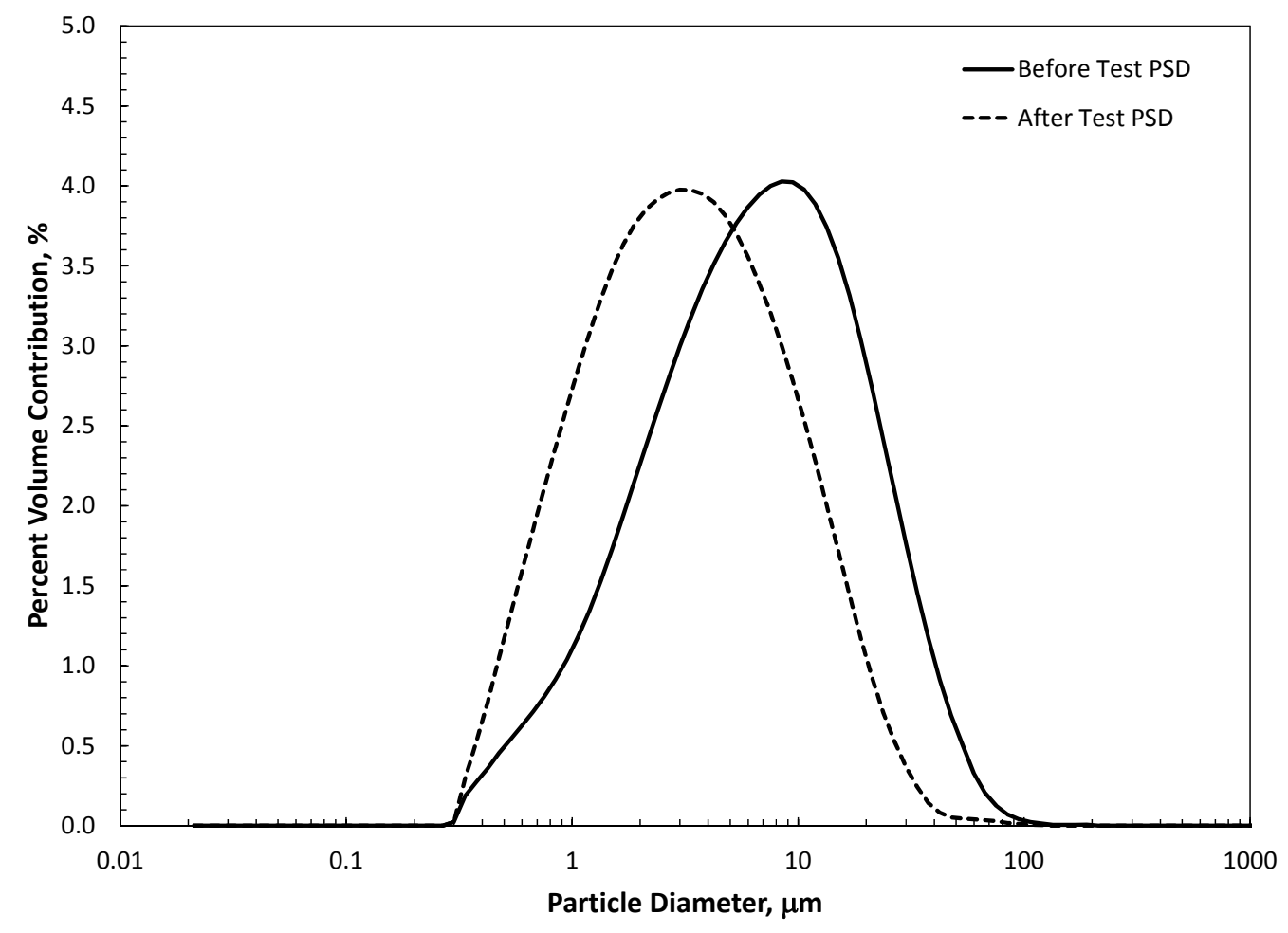

Figure 5.1. Average Particle Size Distribution of PEP Simulant Before and After 100-hour Filter Fouling Tests 
Table 5.2. Particle Size Distribution Table for PEP Simulant Before and After 100-Hour Filter Fouling Tests

\begin{tabular}{ccccccc}
\hline & \multicolumn{3}{c}{ Before 100-hour Test } & \multicolumn{3}{c}{ After 100-hour Test } \\
\cline { 2 - 7 } Percentile & Mean & Mean Error $^{(\mathrm{a})}$ & Variability $^{(\mathrm{b})}$ & Mean & Mean Error $^{(\mathrm{a})}$ & Variability $^{(\mathrm{b})}$ \\
10 & 1.42 & \pm 0.04 & \pm 0.07 & 0.82 & \pm 0.06 & \pm 0.13 \\
50 & 6.7 & \pm 0.5 & \pm 0.8 & 3.2 & \pm 0.4 & \pm 0.8 \\
90 & 24 & \pm 2 & \pm 3 & 12 & \pm 1 & \pm 2 \\
\hline
\end{tabular}

(a) The error in the mean is calculated as $t_{95} \sigma / \sqrt{N}$, where $t_{95}$ is the $95 \%$ confidence limit multiplier (dependent on the number of measurements $N$ used to compute the mean) and $\sigma$ is the sample standard deviation.

(b) The variability is computed as $t_{95} \sigma$, and represents the expected range of measurement variation for any given sample.

Table 5.3. Comparison of Series 1 Mean Values for Physical Properties to Range of PEP Test Values

\begin{tabular}{|c|c|c|}
\hline Physical Property & PEP Range & Series 1 Mean \\
\hline \multicolumn{3}{|l|}{ Rheology ${ }^{(a)}$ : } \\
\hline Bingham Yield Stress, $\mathrm{Pa}$ & $0.052-0.062$ & $0.06 \pm 0.01$ \\
\hline Bingham Consistency, mPa s & $4.77-4.85$ & $3.73 \pm 0.03$ \\
\hline Supernate Viscosity, $\mathrm{mPa} \mathrm{s}$ & $2.82-2.94$ & $2.80 \pm 0.03$ \\
\hline \multicolumn{3}{|l|}{ Density ${ }^{(a)}$ : } \\
\hline Slurry Density, $\mathrm{kg} \mathrm{L}^{-1}$ & $1.26-1.30$ & $1.283 \pm 0.002$ \\
\hline Supernate Density, $\mathrm{kg} \mathrm{L}^{-1}$ & $1.20-1.24$ & $1.2321 \pm 0.0005$ \\
\hline \multicolumn{3}{|l|}{ Solids Concentrations ${ }^{(\mathrm{a})}$ : } \\
\hline Total Solids, wt $\%$ & $31.6-32.7$ & $31.1 \pm 0.1$ \\
\hline Undissolved Solids, wt $\%$ & $6.8-7.0$ & $6.3 \pm 0.2$ \\
\hline Supernate Dissolved Solids, wt\% & $26.8-27.6$ & $26.5 \pm 0.1$ \\
\hline \multicolumn{3}{|l|}{ Unsheared Particle Size Distribution $^{(\mathrm{b})}$ : } \\
\hline $10^{\text {th }}$ Percentile, $\mu \mathrm{m}$ & $1.5-1.8$ & $1.42 \pm 0.04$ \\
\hline $50^{\text {th }}$ Percentile, $\mu \mathrm{m}$ & $6.4-8.5$ & $6.7 \pm 0.5$ \\
\hline $90^{\text {th }}$ Percentile, $\mu \mathrm{m}$ & $20-33$ & $24 \pm 2$ \\
\hline
\end{tabular}

(a) The range of physical properties is derived from measured values associated with PEP Low-Solids Scaling Test \#2 (see Table 4.2 in Billing et al. 2009).

(b) The range of particle size values is derived from PSD results reported for Integrated Tests A and B (Guzman-Leong et al. 2009; Geeting et al. 2009). Unsheared values compared because of difficulty in finding PSD data for matching levels of shear to 100-hour CUF test. 


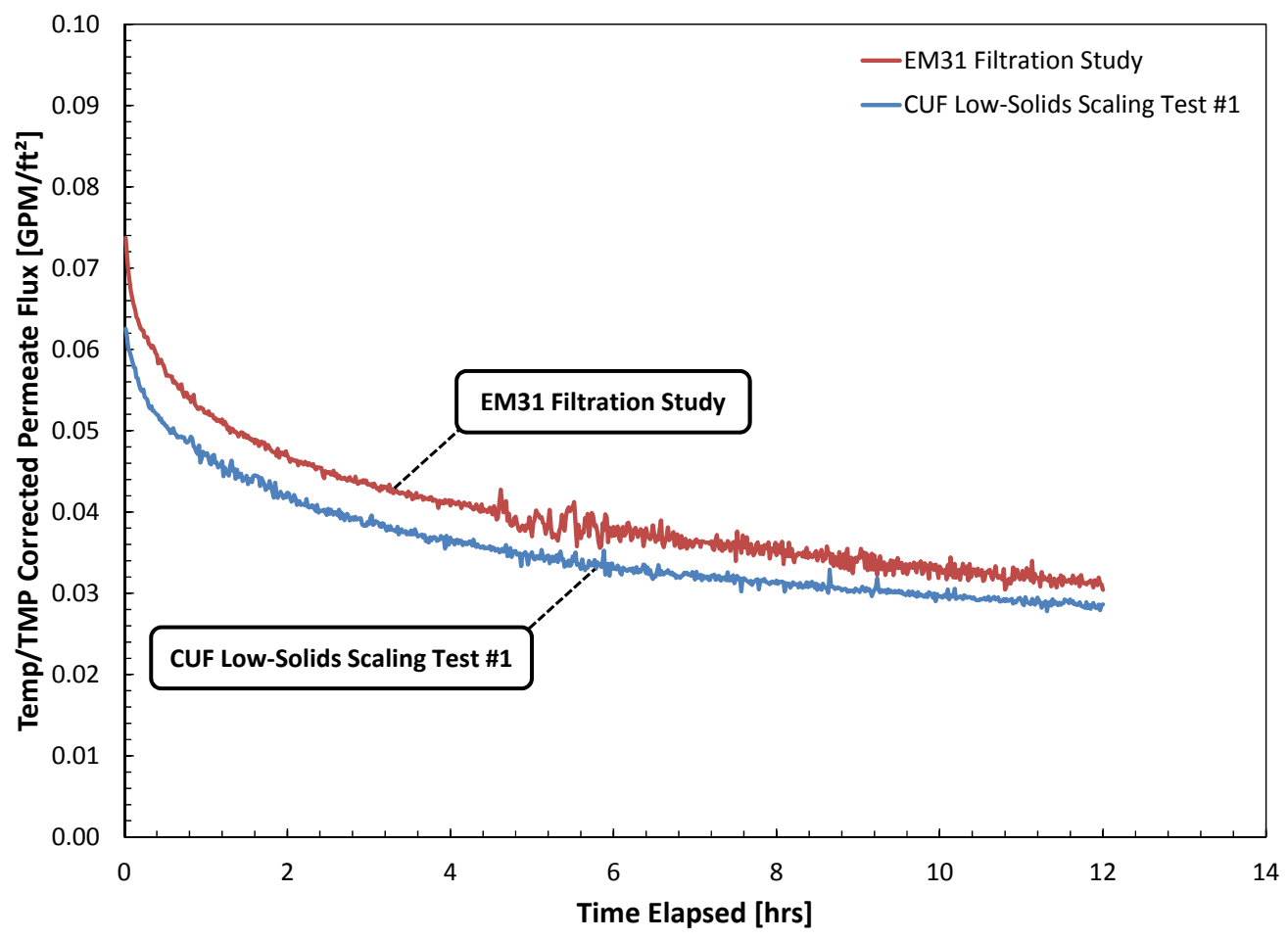

Figure 5.2. Comparison of Filter Flux Decline Profiles for PEP Simulant from the Current EM31 Study and CUF Low-Solids Test \#1 (PEP testing, Daniel et al. 2009b)

As the current studies regard filtration, it is important to verify that the filtration behavior of the PEP simulant used for these tests matches that measured during PEP integrated testing. Figure 5.2 shows a comparison of filter flux decline of the PEP simulant used for the current EM31 filtration study and the flux decline measured during the Low-Solids Scaling Test \#1. Both flux curves were measured on the 2-foot CUF filtration apparatus. The curves show comparable flux decline behavior over the 12-hour period of testing. While the filter curves show a difference in the overall flux magnitude over the test period, this is expected given the slight difference in physical properties between the current simulant and that used in PEP integrated testing (see Table 5.3).

Finally, the slurry volumes for Series 1 testing were selected to be similar with the slurry solids to filter surface area ratios employed in PEP Low-Solids Scaling Tests (Daniel et al. 2009b; Billing et al. 2009). An extensive set of physical property data were not available at the time test volume was selected. As such, the test volume was selected on the basis of a $1.5 \mathrm{~kg} \mathrm{ft}^{-2}$ solids to filter area ratio, a slurry solids concentration of $5-\mathrm{wt} \%$, and a slurry density of $1.28 \mathrm{~kg} \mathrm{~L}^{-1}$. For the 2 -foot filter element, these conditions yield a test slurry volume of approximately $6.2 \mathrm{~L}$. This volume was used for the Series 1 test matrix outlined in Table 3.1 .

\subsection{Test Results and Observations}

In this section, the results of the 100-hour long-time fouling tests are presented and discussed. The results are discussed in three parts: 1) flux decline over the first 100 hours of testing before backpulsing, 2) flux decline after backpulsing, and 3) flux recovery upon backpulsing (i.e., backpulsing effectiveness). In the first subsection, results are further broken down into AV and TMP effects. 
Before continuing, it should be noted that filtration of the PEP simulant was done in recycle mode, whereby all permeate collected was immediately and continuously returned to the slurry reservoir. This mode of operation maintains the slurry at a constant slurry concentration, and declines in filter performance occur solely as a function of time when the CUF is operated at constant TMP and AV. For these reasons, only filter flux as a function of time is presented in this section. All other process conditions are assumed constant until stated otherwise.

Figure 5.3 shows a typical filter flux result for Series 1 testing. The result corresponds to the first 100 hours of test S1T1 (which employs a TMP and AV of $40 \mathrm{psid}$ and $15 \mathrm{ft} / \mathrm{s}$, respectively). The figure presents the filter flux as a function of time scaled linearly and logarithmically. While both figures present the same information, the logarithmically scaled figure is generally more useful for assessing long-time fouling behavior because it 1) clearly presents the decay behavior at all time-scales (whereas the short-time changes are compressed against the y-axis in the linear scale) and 2) more accurately allows assessment of steady-state behavior, particularly when model comparisons are made to the data in Section 8.1. The latter point results from the fact that transport of particles to the surface (and thus fouling dynamics) is controlled by the filter flux. Thus, filter flux decline as a result of fouling slows the rate of filter fouling until a steady-state flux is reached or the flux declines to zero. Because the rate of flux decline slows as fouling continues, slow flux declines can be easily mistaken as steady-state flux conditions when plotted on linear time scales (as shown in Figure 5.3). For the reasons outlined above, this section reports all filter flux results as filter flux as a function of time with time scaled logarithmically.

While the use of the semi-log plots allows easy assessment of long-time scale time changes, care should be taken when using the plots to infer rate of change in filter flux. As seen on the normal time scale axis, the rate of filter flux decline slows throughout the filtration process. In all test cases, the filter flux declines rapidly at small times relative to long-time declines, which occur slowly. Use of the log-scale for the time axis equalizes the rate of change to an extent, making it appear that flux changes more slowly during the initial periods and more quickly during the final periods than it does on a normal time axis. For this reason, attention must be paid to the time-scales involved when comparing change in flux, as the time scale used for data presentation is not linear.

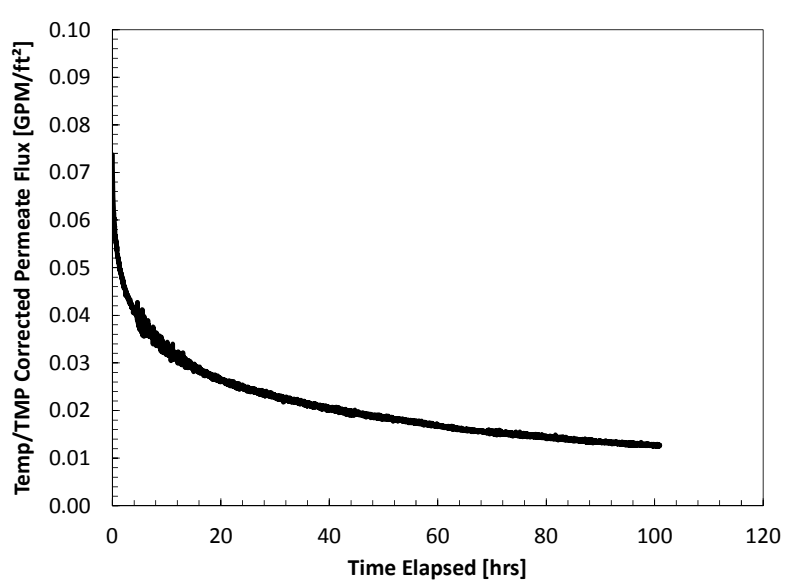

(a)

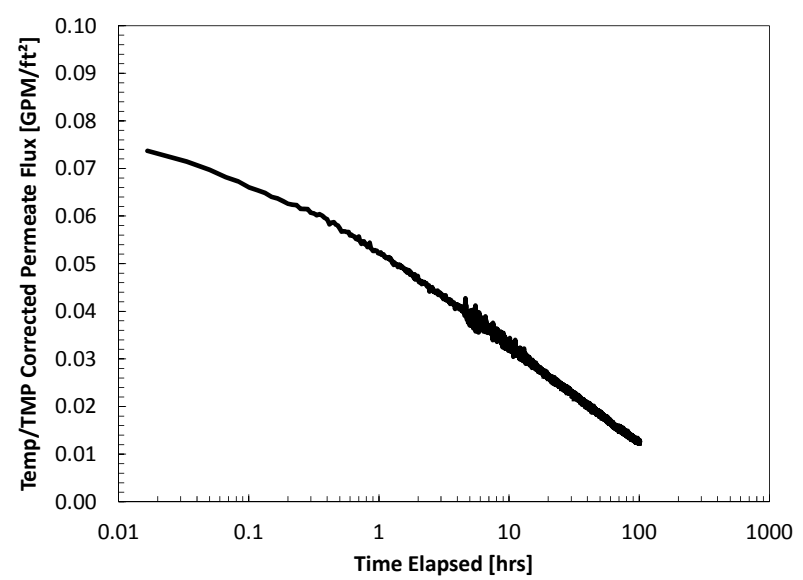

(b)

Figure 5.3. Typical Series 1 Filtration Test Result with Time Scaled Linearly (a) and Logarithmically (b) 


\subsubsection{Flux Behavior Before Backpulsing}

The results of long-time fouling tests during the first 100 hours of filtration are presented in Figure 5.4 and Figure 5.5, which show the impact of changing axial velocity and transmembrane pressure on the filter flux magnitude and rate of decline. As discussed above, tests results are plotted as filter flux versus time with time scaled logarithmically to accentuate long-time changes in flux.

In all tests, the filter flux declines with time, indicating that the simulant solids are fouling the filter element (either through depth fouling or cake formation). The overall reduction in flux that occurs during the tests is significant. The flux at 100 hours ranges from 10 to $30 \%$ of the initial filter flux. Likewise, the filter flux declines continuously throughout the test duration and does not appear to reach a steady-state for any of the tests. At times greater than 1 hour, filter flux appears to decrease linearly with the logarithm of time. This trend continues until the end of the test at 100 hours, where the filter flux for most tests approached $10 \mathrm{~mL} / \mathrm{min}$ (the lower limit of accuracy for the CUF permeate production rate flow meter). As such, the existence of a steady-state flux cannot be assessed from the current data sets. While the data strongly suggest that the flux will continue to decline at filtration times greater than 100 hours, the ultimate fate of filter flux cannot be determined. If the flux behavior is extrapolated using the logarithmic trend observed in the data, a zero flux condition occurs for all tests. For S1T1, such extrapolation indicates zero flux (complete filter blockage) at $\sim 400$ hours. However, the reasonableness of such an extrapolation is suspect, since cross-flow filtration typically yields a steady-state flux at much shorter times (ten hours or less) and dead-end filtrations asymptote to zero flux at infinite filtration time. Indeed, analysis of the filter flux behavior in Section 1 indicates that the behavior is consistent with the fouling mechanisms encountered in dead-end filtration. This indicates that the fouling layer is beyond the reach of the scouring action of axial velocity (and may indicate penetration of particles and particle agglomerates into the porous substructure of the filter element). It can be postulated then that the flux will asymptotically decay to zero rather than reaching zero flux at finite time or achieving steady-state. Ultimately, the current tests provide no direct evidence as to the ultimate fate of filter flux. If the steady-state or zero flux condition is to be properly assessed, longer time filtration tests (e.g., 400-hour tests) may be required.

The suggestion that a steady-state flux will not be achieved may initially seem anomalous for the cross-flow filtration configuration, but it is not entirely unexpected when the filter pore dimensions are considered against the size of waste particles contained in the slurry. Comparison of hydrodynamic pore diameter of 1 to $3 \mu \mathrm{m}$ with the PEP waste simulant particle size distribution (Table 5.2) indicates that for the initial PSD , 5 to $25 \%$ of waste particles by volume are smaller than or equal to the estimated pore size of the filter medium. When the final PSD is considered, the volume percent of simulant particles smaller than or equal to the estimated filter pore size increases to 15 to $45 \%$. This has significant implications for the filtration process. Particles that fall below the pore size have the potential to infiltrate and block filter pores. If particles infiltrate the porous structure, then they are beyond the influence of the axial cross-flow, which transports the particles from the filter surface into the bulk flow. As such, the benefits of cross-flow filtration are lost for the portion of the waste particles that infiltrate the porous medium.

Figure 5.4 illustrates the impact of AV on filter flux decline. As expected, higher axial velocities yield higher filter flux relative to lower axial velocities. This expectation is based on the fact that higher axial velocity should yield more effective scouring of any cake layer that forms while also limiting particle-pore interactions through inertial arguments. While the differences in flux magnitude with axial velocity are small at the beginning of the test, they persist throughout the test and become more 


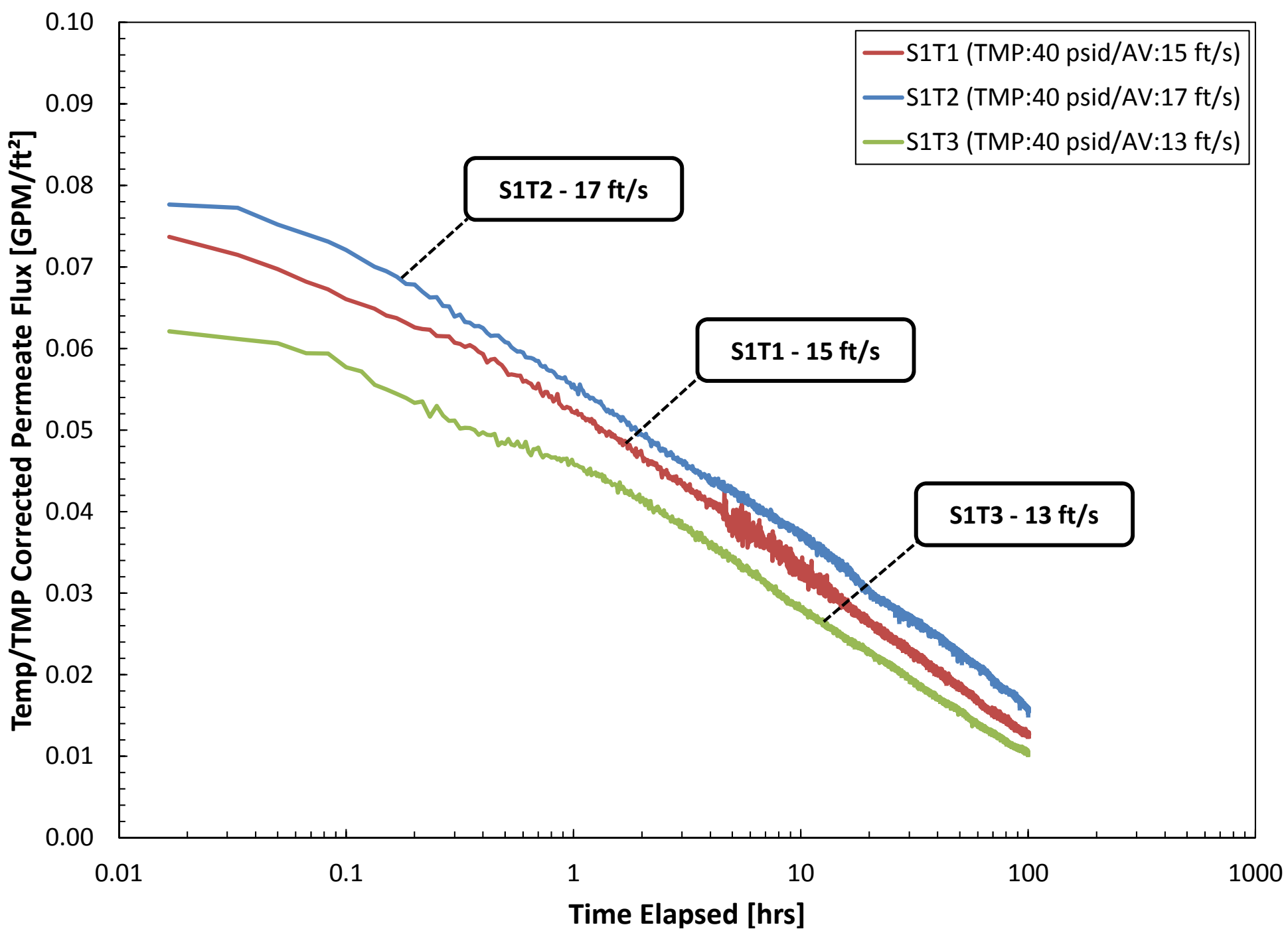

Figure 5.4. Corrected Permeate Flux Decline Over Initial 100-Hours at Different Axial Velocities 


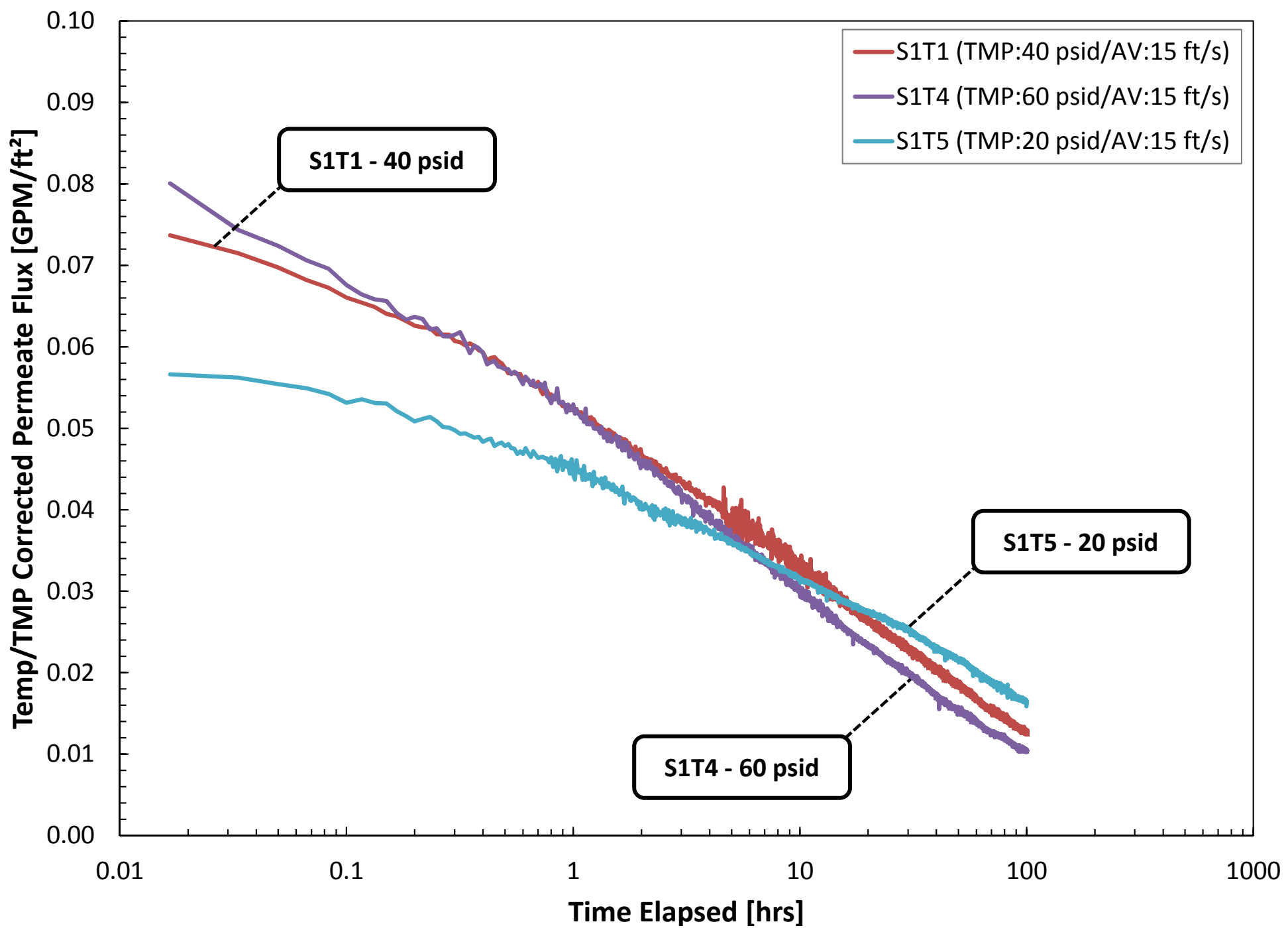

Figure 5.5. Corrected Permeate Flux Decline Over Initial 100-Hours at Different Transmembrane Pressures 
significant (in terms of absolute flux ratios) as filtration progresses. It is interesting to note that the differences in flux with axial velocity exist even at the start of filtration. However, since the initial flux is much smaller than the clean water flux, it is likely that significant flux decline occurs either before the start of filtration or within the first few seconds of filtration. While axial velocity appears to impact the flux magnitude, it does not appear to appreciably change the slope of the curves, at least when plotted as the logarithm of time. This is illustrated by the fact that the 13,15, and $17 \mathrm{ft} / \mathrm{s}$ filter flux curves run parallel for the duration of the test. Overall, the AV studies indicate that higher axial velocities can yield improvements in filter throughput.

Figure 5.5 shows the impact of TMP on the filter flux behavior during the initial 100 hours of continuous filtration testing. The results suggest that increased TMP yields higher initial filter flux values. In the data shown, both 40 and 60 psid filter tests show higher flux over the first 5-20 hours of filtration testing relative to $20 \mathrm{psid}$. In addition, the results also indicate that increases in TMP beyond 40 psid may not provide an increase in filter flux. Comparison of the filtration behavior over the entire duration of testing indicates that higher TMP increases the rate of filter fouling. That is, the 60 psid filter flux curve shows the most rapid decrease in filter flux whereas the 20 psid curve shows the slowest decline. This trend continues throughout the entire test duration, such that by the end of the 100-hour test, the 20 psid filter curve shows the best filter performance and the 60 psid curve shows the lowest flux. This observation is significant, as it indicates that low operating TMPs yield better filter throughput relative to high TMPs when the filter is operated for long-times without backpulsing. This behavior may be important for process optimization in filtration operations where backpulsing is avoided because it yields an increase in the rate of irreversible filter fouling (such as that observed in low-solids scaling tests in Daniel et al. 2009b).

\subsubsection{Flux Behavior After Backpulsing}

Figure 5.6 shows the flux behavior with time following the backpulse at 100 hours. As before, the results are presented as filter flux as a function of time. The flux magnitude following backpulsing is similar for all tests. Fouling of the filters is evident, as the post-backpulse flux declines with filtration time. However, beyond this decline, there are no strong trends with operating conditions during the first hour of filtration after the backpulse. The flux curves appear to diverge slightly after approximately 3 hours of post-backpulse testing. The trends observed after 3 hours at different AV and TMP appear to follow the same trends observed before backpulsing; however, the divergence observed between operating conditions is not yet large enough to make this assertion with confidence.

An example of the flux behavior before and after backpulsing is shown in Figure 5.7, which compares flux behavior with time before and after backpulsing for S1T1. This result is typical, and is consistent with the behavior observed for all Series 1 tests. Here, the filter flux attained after backpulsing is significantly less than the initial flux. This difference indicates that a portion of the flux loss that occurred during the first 100 hours cannot be recovered through backpulsing. This irreversible loss affects backpulse efficiency, which will be discussed in more detail in Section 5.2.3.

Because the test duration for post-backpulse operations was limited to 3 to 4 hours, it is not possible to assess the ultimate fate of post-backpulse flux. Although the slope (i.e., the rate of flux decline) for the post-backpulse flux is less than that of the initial flux over similar elapsed time, the slower rate of fouling is most likely a result of lowered filter flux (and lowered solids transport to the filter surface) rather than a fundamental change in the way the filter fouls. This lower slope is not expected to persist. Indeed, the current expectation is that the post-backpulse flux will asymptote with the pre-backpulse flux curve at long- times. However, additional testing is needed to confirm this expectation. 


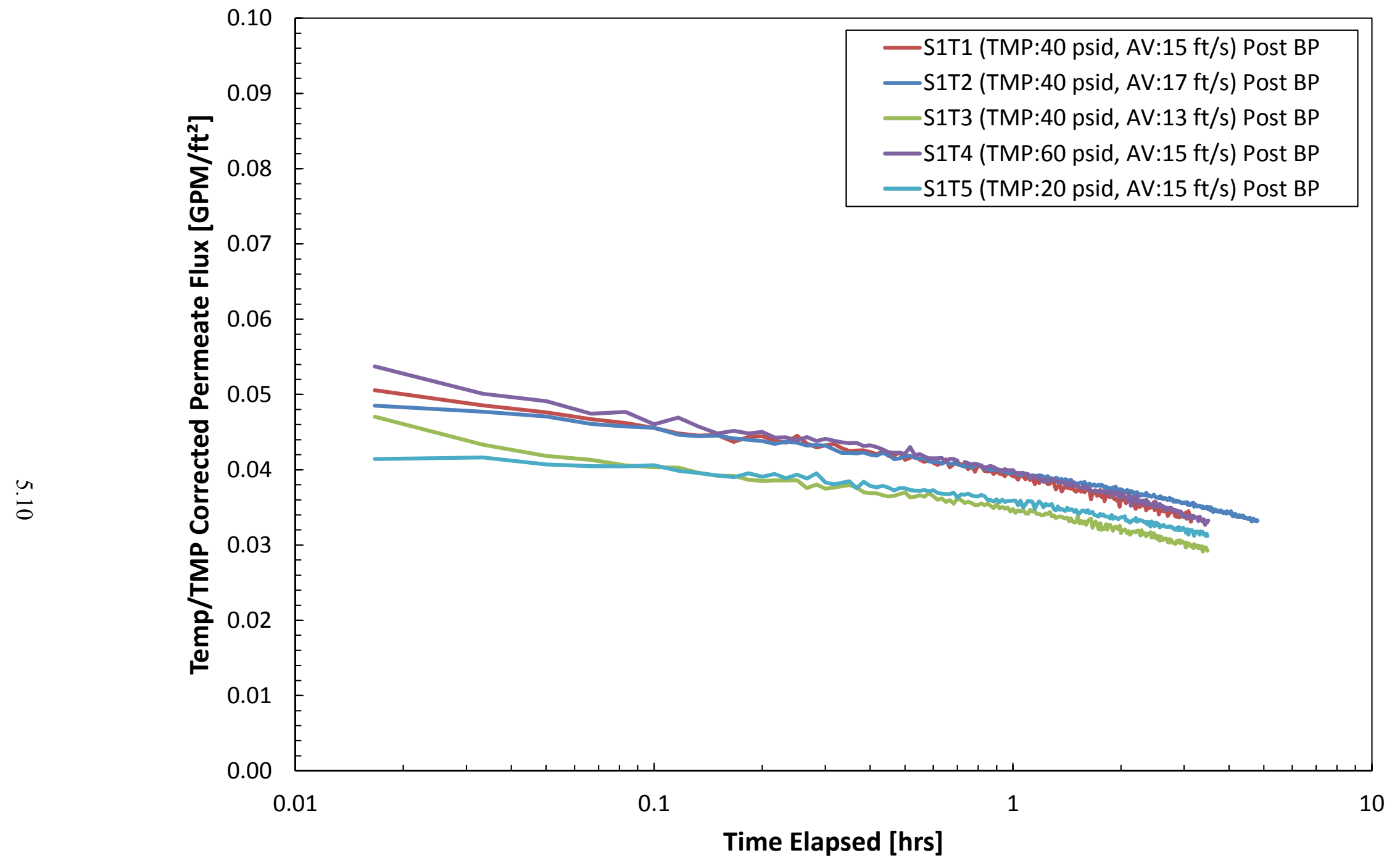

Figure 5.6. Corrected Permeate Flux Decline Following Backpulse at 100-Hours of Filtration Time 


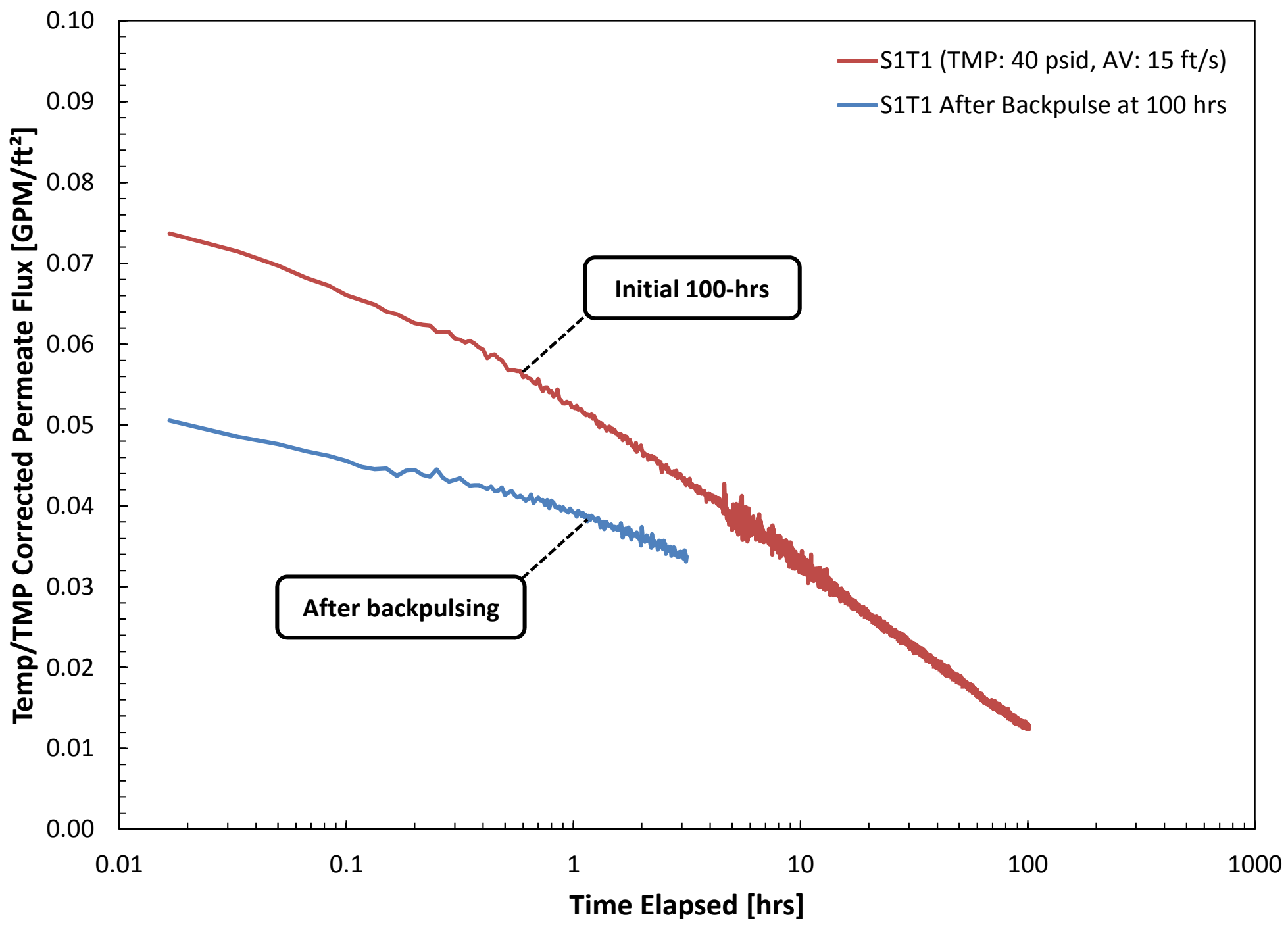

Figure 5.7. Comparison of Flux Behavior Before and After Backpulsing (Test S1T1) 


\subsubsection{Backpulsing Effectiveness}

As stated in the previous section, the post-backpulse flux observed during Series 1 testing was always significantly lower than the initial flux. On the other hand, the flux recovered after backpulsing was also higher than the flux at 100 hours. As such, while backpulse was effective at restoring filter flux, it could not restore the flux entirely to its initial level. As such, irreversible flux loss occurs either during filtration or as a result of backpulsing.

To assess the effectiveness of backpulsing at restoring filter flux after 100 hours of filtration, the flux recovered after backpulsing is compared to the initial filter flux measured at the start of the 100-hour test period. This comparison is shown in Figure 5.8 and Table 5.4. Here, the initial, pre-backpulse, and post-backpulsing fluxes are averaged over 1 hour to reduce the impact of any rapid changes associated with process start-up or backpulsing. Figure 5.8 clearly illustrates the previous assertion that post-backpulse fluxes are lower than initial fluxes and also highlights the similarity in post-backpulse flux. Because of the similarity in flux following backpulsing, it can be speculated that the process that yields irreversible fouling is similar for all tested operating conditions, such that it yields the same degree of fouling. The process may be similar to that of filter conditioning employed in the low-solids scaling tests at PEP (Daniel et al. 2009b). In PEP, a disparity in the initial flux measured on CUF and PEP filtration systems for the same PEP simulant tested herein was reduced by repeatedly backpulsing the filters to condition them against the slurry solids. The results presented in Daniel et al. (2009b) suggested that repeated backpulsing of the filters caused irreversible fouling. From the current result, it is less clear if the single series of backpulses at 100 hours, the 100-hour filtration period, or both are the primary contributors to irreversible fouling.

Table 5.4 represents the results shown in Figure 5.8 in tabular form. It also presents a measure of backpulsing efficiency in terms of the flux ratio, which is the post-backpulse flux divided by the initial flux. This measure of effectiveness does not account for the existence of a steady-state flux. In all cases, backpulsing only restores $\sim 25 \%$ of the initial flux. The ratio appears to vary slightly with test condition. However, because the most effective backpulses occurred for tests with the lowest initial flux, it is difficult to decouple pre-test steps that could have impacted the initial flux from operating condition effects for this measure of backpulse effectiveness without replicate testing (i.e., repeat testing at the same operating AV/TMP).

Overall, the test results shown in Figure 5.8 and Table 5.4 raise concerns about backpulsing effectiveness. Figure 5.8 clearly shows that while backpulsing does significantly restore flux, there is also a significant amount of irrecoverable flux loss that occurs. Based on the current results, it cannot be determined if irrecoverable fouling occurs during the 100 hours of continuous filtration or because of the series of backpulses performed at 100 hours. While the degree of irrecoverable fouling for the current tests has been quantified ( $25 \%$ loss) in Table 5.4, the overall degree to which the filter can irreversibly foul cannot be determined. Specifically, there is concern that irreversible fouling could continue indefinitely (either as a result of repeated backpulsing or filtration) until flux can no longer be recovered through backpulsing. Additional testing is needed to confirm if irreversible fouling (filter conditioning) has a steady-state value. 


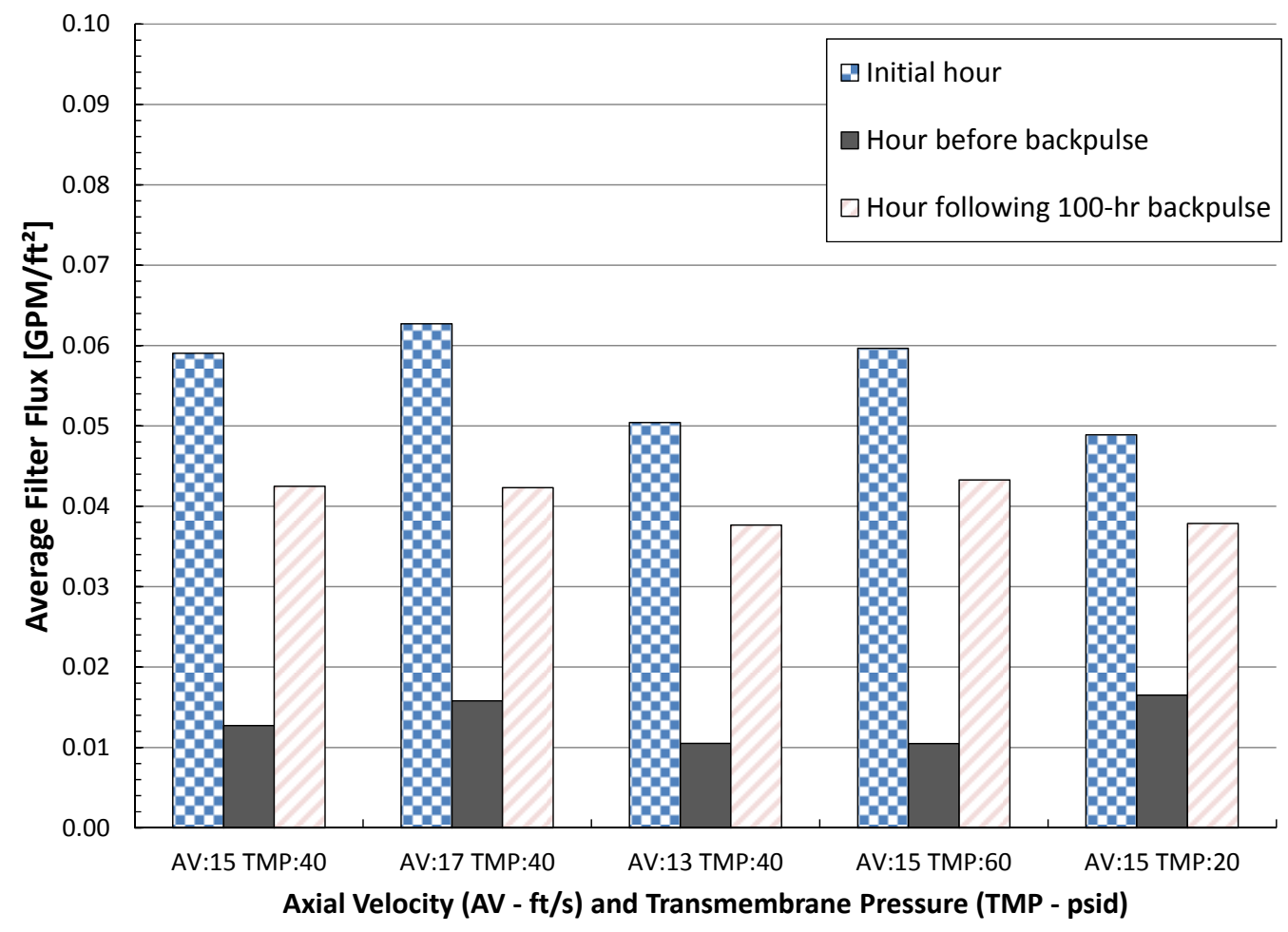

Figure 5.8. Comparison of Initial Flux at Start of Test and Flux Immediately Following Backpulsing at 100-Hours (averaged over 1 hour of filtration)

Table 5.4. Comparison of Initial Flux and Flux After Backpulsing

\begin{tabular}{cccccc}
\hline & $\begin{array}{c}\text { Av } \\
\text { Test }\end{array}$ & $\begin{array}{c}\text { TMP } \\
{[\mathrm{ft} / \mathrm{s}]}\end{array}$ & $\begin{array}{c}\text { Average Flux } \\
1^{\text {st }} \text { hour after start } \\
{\left[\mathrm{GPM} / \mathrm{ft}^{2}\right]}\end{array}$ & $\begin{array}{c}\text { Average Flux, } \\
1^{\text {st }} \text { hour after final BP } \\
{\left[\mathrm{GPM} / \mathrm{ft}^{2}\right]}\end{array}$ & Ratio $^{(\mathrm{a})}$ \\
\hline S1T1 & 15 & 40 & 0.0590 & 0.0425 & 0.720 \\
S1T2 & 17 & 40 & 0.0627 & 0.0423 & 0.675 \\
S1T3 & 13 & 40 & 0.0504 & 0.0377 & 0.747 \\
S1T4 & 15 & 60 & 0.0596 & 0.0433 & 0.726 \\
S1T5 & 15 & 20 & 0.0489 & 0.0379 & 0.775 \\
\hline (a) The ratio of flux after backpulsing to the initial flux - a measure of irreversible flux loss.
\end{tabular}

(a) The ratio of flux after backpulsing to the initial flux - a measure of irreversible flux loss.

\subsection{Series 1 Test Conclusions}

Series 1 testing examined filtration behavior for the waste simulant slurry used for PEP testing over times much longer than previously tested. Specifically, the simulant slurry was filtered for 100 hours in recycle mode (constant solids concentration) without filter backpulsing. At the end of the 100 hour-test period, the filter was backpulsed to assess the effectiveness of backpulsing in restoring filter flux. These tests were run for a matrix of different AV and TMP to assess the impact of operating conditions on long-time filter flux behavior. The major findings of Series 1 testing are as follows: 
- no steady-state filter flux was observed - for all operating conditions, the filter flux continuously declined and did not reach a steady-state filter flux. Filter flux decline slowed dramatically at long-time; however, the observed functionality appears linear with the logarithm of time. Extrapolation of the observed behavior indicates that the filter will block completely (i.e., exhibit zero flux) at finite time. The appropriateness of this extrapolation is suspect and additional modeling of long-time fouling functionality against literature models is recommended (and done in part in Section 1 of this report). Ultimately, the current results suggest potential difficulty for filtration operations at greater than 100 hours, as continued flux decline will significantly impact filter throughput unless counter-balanced by flux recovery operations.

- higher axial velocity yields higher filter flux - use of high axial velocity improved filter flux relative to tests using lower axial velocity for the entire duration of the test. This improvement is consistent with expectations based on improved scouring of particles from the surface of the filter and reduced particle-pore interactions (because of increase axial inertia) at higher axial velocities.

- lower transmembrane pressure yields more efficient long-term operations - the test results show that while high transmembrane pressure yields higher initial filter fluxes, it also accelerates the rate of filter fouling significantly when the filter is operated continuously without backpulsing. On the other hand, operating at low transmembrane pressures yields lower initial filter fluxes, but the filter does not foul as strongly during non-backpulsed operations. For the current results, operating the filter at 20 psid yielded lower fluxes until 5-20 hours of filtration time, after which 20 psid showed the highest filter flux for the remainder of testing. This result suggests that operating at lower transmembrane pressure may provide improved filter throughput if the filters are operated for long times without backpulsing or cleaning.

- Backpulsing does not completely restore flux - an approximately $25 \%$ flux loss occurred for all tests that could not be corrected by backpulsing. This poses significant uncertainty with respect to the long-time effectiveness of backpulsing. Specifically, there is concern that irreversible fouling could continue indefinitely (either as a result of repeated backpulsing or filtration) until flux can no longer be recovered through backpulsing.

The findings outlined above have significant impact with respect to the performance high-level waste filtration operations. First, the lack of observed filtration steady-state after 100 hours has significant implications on the throughput of filtration operations greater than 100 hours. Indeed, the current tests suggest that the filter will dead-end (go to zero flux) at finite time, necessitating flux recovery operations such as backpulsing or chemical cleaning. Extrapolation of filter flux results for one set of operating conditions indicates that the zero-flux time is $\sim 400$ hours. As such, PNNL recommends a 400 -hour filter test to verify the observed long-time flux behavior.

The long-time test results also indicate a strong need for a better understanding of flux recovery operations. Backpulsing was shown to effectively restore a significant portion of the filter flux. However, a portion of the flux $(\sim 25 \%)$ could not be recovered by the single set of backpulses applied after 100 hours of continuous filtration. Irreversible flux loss with respect to backpulse is cause for concern, as the underlying mechanisms are not well understood. Additional testing is needed to confirm if irreversible fouling (filter conditioning) has a steady-state value or will continue to degrade flux recovered upon backpulsing. Because the information derived from additional backpulsing testing might be used to optimize backpulse frequency or decide the use of backpulsing at all, the findings would also feed into optimization of process TMP and AV. 
Finally, the long-time AV and TMP results are significant in that they provide insight into how the filtration process can be optimized to improve filter throughput. The throughput results for the first 100 hours of testing are shown in Table 5.5. The results are surprising, as they show that for 100 hours of undisturbed filtration, throughput can be improved by increasing axial velocity and lowering transmembrane pressure. This contrasts with previous short-time test results, which indicated that increased axial velocity and transmembrane pressure were needed to improve throughput. As such, the current tests illustrate the importance of understanding fouling dynamics with respect to the selected operating conditions as non-obvious process optimizations (such as lower transmembrane pressure) may result in better filter performance.

Overall, the Series 1 test results highlight the need for predictive models that appropriately capture both long-term fouling dynamics and how those dynamics change with operating conditions such as axial velocity and transmembrane pressure. This, combined with a predictive understanding of both reversible and irreversible fouling with respect to backpulsing, can lead to better process optimization and higher filter throughput.

Table 5.5. Filter Throughput Results (Volume Permeate Filtered) During First 100 Hours of Series 1 Testing

\begin{tabular}{|c|c|c|c|}
\hline Test & $\begin{array}{l}\mathrm{AV} \\
{[\mathrm{ft} / \mathrm{s}]}\end{array}$ & $\begin{array}{c}\text { TMP } \\
\text { [psid] }\end{array}$ & $\begin{array}{c}\text { Volume Permeate Produced } \\
\text { During First } 100 \text { hours } \\
{[\mathrm{L}]}\end{array}$ \\
\hline S1T1 & 15 & 40 & 128 \\
\hline S1T2 & 17 & 40 & 151 \\
\hline S1T3 & 13 & 40 & 107 \\
\hline S1T4 & 15 & 60 & 110 \\
\hline S1T5 & 15 & 20 & 140 \\
\hline
\end{tabular}





\subsection{Series 2 Testing Results}

In Series 2 testing, several cross-flow filtration tests were performed to assess 1 ) the propensity of individual PEP simulant components to foul CUF filter elements and 2) the impact of suspending phase chemistry on the filtration performance of those components. The overall goal of these tests is to provide insight into which simulant component drive declines in filter performance with time and the efficacy of backpulsing for maintaining acceptable filter fluxes over time. The test approach for Series 2 is described Section 3.2. In this section, the results for Series 2 are presented and discussed in detail. The results are broken into two separate categories. These are:

- component baseline testing - where the filter performance of individual PEP simulant components was assessed under fixed suspending phase chemistry, AV, and TMP conditions. Component baseline testing is comprised of data taken in Segments 2 through 4 of Series 2 testing.

- pH dependence testing - where the filter performance of the same PEP simulant components was assessed with changing $\mathrm{pH}$. Changing $\mathrm{pH}$ is expected to change the surface charge of both filter element and solid particles. At high $\mathrm{pH}$, filter and particle are expected to be similarly (negatively) charged. The same holds for low $\mathrm{pH}$, where filter and particle both are expected to share a positive charge. At intermediate $\mathrm{pH}$, there can be a potential for dissimilar charges (e.g., a positive particle and a negative filter surface), which could yield strong, irreversible sticking of particles to the filter surface. In these intermediate regions, strong fouling of the filter is expected. $\mathrm{pH}$ dependence testing is comprised of data taken in Segment 5 of Series 2 testing.

\subsection{Component Baseline Testing}

Component baseline testing examined three simulant solids: boehmite, iron oxy-hydroxide ( $\mathrm{FeOOH})$, and chrome oxy-hydroxide $(\mathrm{CrOOH})$. For testing with boehmite solids, both coarse boehmite and fine boehmite solids were tested separately to examine the impacts of particle size on the filtration behavior.

\subsubsection{Component Characterization}

The particle size distribution of the four particulate systems tests is given in Figure 6.1. These results are also given in tabular form in Table 6.1. It should be noted that the results are based on an assumed particle refractive index of 2.24 and absorption index of 1 . These parameters were employed to provide a consistent optical property basis for all systems so that they can be compared to the PSD of mixed particles systems (e.g., the combined PEP simulant shown in Figure 5.1). The reported PSD data represent the test suspensions after loading into the CUF slurry reservoir but before testing. It is expected that, as with the PEP simulant results shown in Figure 5.1, CUF processing will effect a reduction in the overall size of suspension particles; however, post processing samples were not taken to confirm this. Based on this expectation, estimates of potential infiltration based on initial PSDs, which are discussed on the following pages, should be lower than actually realized in testing. 

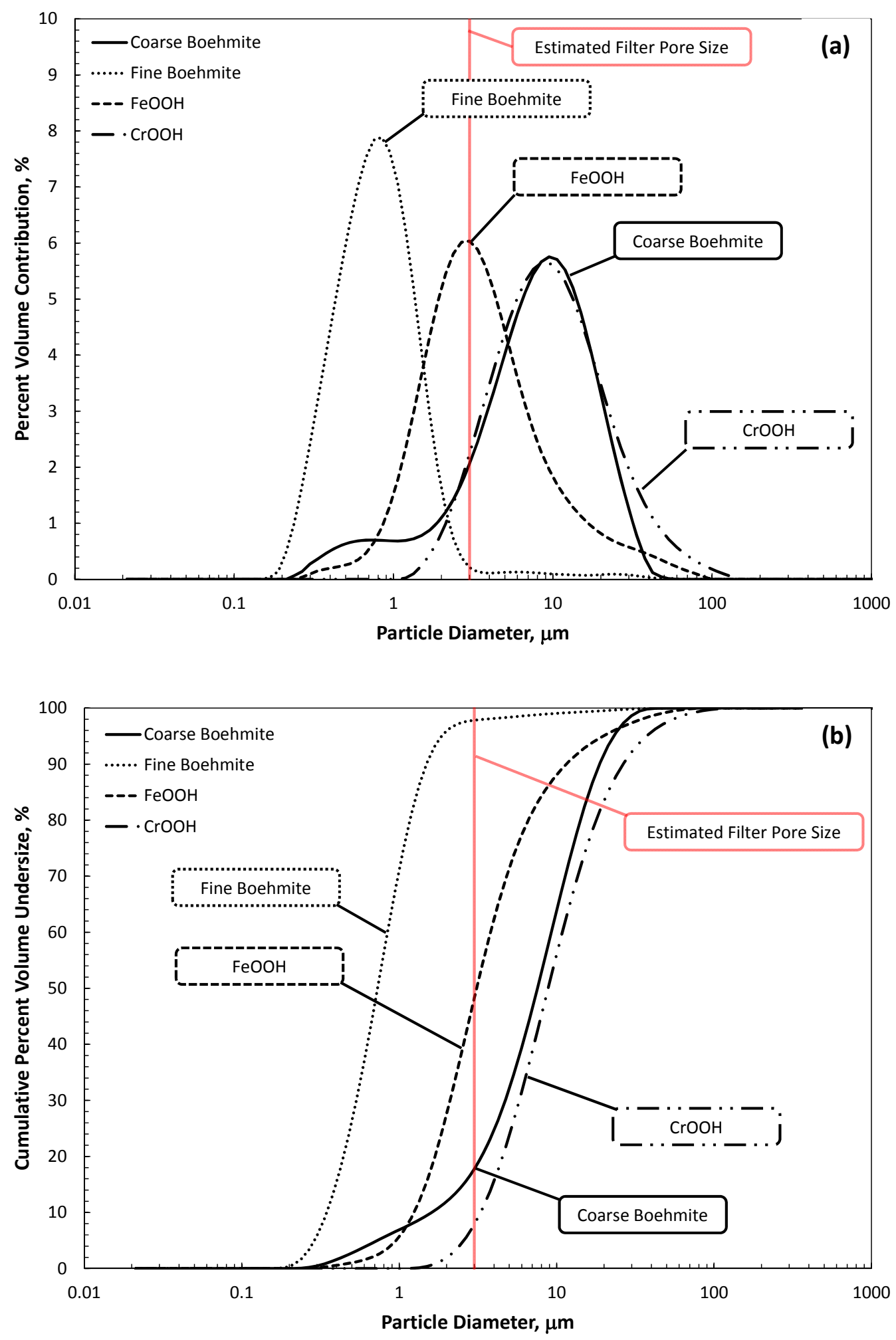

Figure 6.1. Differential (a) and Cumulative (b) Particle Size Distribution of Materials Used in Component Testing (unsonicated pre-test particle dispersion) 
Table 6.1. Tabular Particle Size Distribution of Materials Used in Component Testing (unsonicated pre-test particle dispersion)

\begin{tabular}{ccccc}
\hline & & \multicolumn{2}{c}{ Particle Diameter, $\mu \mathrm{m}$} & \\
Percentile & Coarse Boehmite & Fine Boehmite & FeOOH & CrOOH \\
\hline 1 & 0.37 & 0.26 & 0.52 & 1.9 \\
5 & 0.77 & 0.31 & 1.0 & 2.7 \\
10 & 1.7 & 0.37 & 1.3 & 3.5 \\
20 & 3.5 & 0.47 & 1.8 & 4.8 \\
25 & 4.3 & 0.52 & 2.0 & 5.5 \\
30 & 5.0 & 0.57 & 2.2 & 6.1 \\
40 & 6.4 & 0.66 & 2.7 & 7.6 \\
50 & 8.0 & 0.77 & 3.3 & 9.3 \\
60 & 9.8 & 0.89 & 4.0 & 11 \\
70 & 12 & 1.0 & 5.1 & 14 \\
75 & 13 & 1.1 & 5.9 & 16 \\
80 & 15 & 1.2 & 7.0 & 19 \\
90 & 20 & 1.6 & 12 & 28 \\
95 & 24 & 2.0 & 21 & 39 \\
99 & 33 & 10 & 48 & 74 \\
\hline
\end{tabular}

Section 5.2.1 discussed the potential of PEP simulant particles to infiltrate the filter element porous substructure by comparing particle size to the estimated hydrodynamic diameter of 1 to $3 \mu \mathrm{m}$ for the filter medium. It is useful to perform the same analysis for component testing to assess their ability to infiltrate the filter medium. Before presenting this assessment, it should be noted that the size of particles relative to the filter element is only one of many parameters the influence pore-medium interactions (also see Table 6.2).

The coarse boehmite is the baseline boehmite component for the PEP simulant. It has a medium particle diameter of $8.0 \mu \mathrm{m}$ (based on volume distribution and the assumed optical properties). Based on the upper pore size limit of $3 \mu \mathrm{m}$, approximately $\sim 18 \%$ of the boehmite by volume has a pore size equal to or lower than that of the filter medium. The fine boehmite has a median diameter of $0.77 \mu \mathrm{m}$ and consists of $98 \%$ particles by volume that fall below the $3 \mu \mathrm{m}$ effective hydrodynamic pore diameter. This material was selected to study the effect of size on the filtration behavior. In addition, the majority of particles in the fine boehmite fall in the colloid range, and as such, it is expected that their behavior should be strongly impacted by suspending phase chemistry. In contrast, the coarse boehmite only shows a fraction of particles in the colloidal size range, and as such, their filtration behavior should be much less sensitive to suspending phase chemistry. Overall, relative to the coarse boehmite system, the fine boehmite particle system was expected to 1) plug the filter pores (yielding a significant decrease in filter performance over time) given size arguments and 2) show significant changes in filtration behavior with changing $\mathrm{pH}$.

$\mathrm{FeOOH}$ is present in the baseline PEP simulant and is an amorphous (non-crystalline) solid. In this respect, it is different than either boehmite or gibbsite waste simulant components, both of which are crystalline. PSD measurements of $\mathrm{FeOOH}$, shown in Figure 6.1, evidence a broad particle size distribution that ranges from $\sim 0.3$ to $100 \mu \mathrm{m}$. Approximately $48 \%$ of the FeOOH solids by volume fall below the effective upper pore diameter of $3 \mu \mathrm{m}$, and as such, a significant portion of boehmite solids have the potential of infiltrating the filter pores. 
Table 6.2. Estimate of Potential Volume of Particle that Can Infiltrate and Foul CUF Filter Element Pores

\begin{tabular}{cc}
\hline Component & Percent Volume Below $3 \mu \mathrm{m}^{(\mathrm{a})}$ \\
\hline Coarse Boehmite & $18 \%$ \\
Fine Boehmite & $98 \%$ \\
FeOOH & $48 \%$ \\
$\mathrm{CrOOH}$ & $8 \%$ \\
\hline
\end{tabular}

(a) $3 \mu \mathrm{m}$ is the estimated upper bound of hydrodynamic pore radius of the CUF filter element. The percent of material that falls below this provides an estimate of the amount of material that can infiltrate and foul the porous filter element and is based on the PSD listed in Table 6.1.

Like FeOOH solids, $\mathrm{CrOOH}$ solids are amorphous. However, $\mathrm{CrOOH}$ is not a baseline PEP component, but is a simulant additive introduced to the simulant slurry after caustic leaching but before oxidative leaching. It is intended to simulant $\mathrm{Cr}$ bearing species found in actual waste. Unfortunately, if $\mathrm{CrOOH}$ (which simulant Cr-bearing solids in actual waste) is included in the baseline formulation, it is prematurely leached from the simulant by caustic leaching. It was included in the current component test matrix because it is amorphous (like $\mathrm{FeOOH}$ ) and is a simulant of solids species found in actual wastes intended for treatment at WTP. PSD measurement for $\mathrm{CrOOH}$ indicate that the particles range from 1 to $100 \mu \mathrm{m}$ in diameter. Based on these PSD data, approximately $8 \%$ of the $\mathrm{CrOOH}$ solids fall below the $3 \mu \mathrm{m}$ cut-off for the filter. This suggests that $\mathrm{CrOOH}$ solids will not interact strongly with the filter elements porous substructure.

\subsubsection{Component Test Results}

As discussed in Section 3.2 of this report, each component baseline test consisted of three 12-hour test segments and employed a 0.1 -vol\% suspension of the test component in $0.05 \mathrm{M} \mathrm{NaNO}_{3}$ solutions with a pH of 10 (achieved by $\mathrm{NaOH}$ addition). During the first 12 hours, the filter was operated for 12 hours without backpulsing. During the second 12-hour segment, the filter was backpulsed every 0.5 hour. And during the final 12-hour segment, the filter was operated again without backpulsing. For all test segments, the filter was operated in a continuous recycle filtration mode where all permeate collected is immediately and continuously returned to the slurry reservoir such that a constant slurry concentration of $0.1 \%$ solids by volume was maintained throughout the duration of the 36-hour test. All tests were run at an AV of $15 \mathrm{ft} / \mathrm{s}$ and a TMP of 40 psid using a 2-foot tubular filter element.

Figure 6.2 shows the results for baseline component testing of both coarse and fine boehmite solids. The initial 12-hour hold period is shown in blue, the 12-hour period of backpulsed operations is shown in red, and the final 12-hour hold is shown in green. During both initial and final hold periods, coarse boehmite shows a decline in filter flux with time that is typical (at least qualitatively) of waste simulant suspensions. Relative to coarse boehmite, both the rate and overall magnitude of flux decline observed for the fine boehmite is smaller; that is, fine boehmite appears to foul more slowly and has a reduced propensity for flux decline.

Both fine and coarse boehmite show filter flux that is an order of magnitude higher than is typically observed for the PEP waste simulant sludge (which yields flux in the 0.01-0.05 GPM/ $\mathrm{ft}^{2}$ range). These high flux magnitudes are notable, at least with respect to fine boehmite, as it illustrates that factors other than particle size can strongly influence flux. For the current results, it appears that both coarse and fine 
boehmite do not infiltrate the filter medium, either as a result of unfavorable filter-particle interactions or particle aggregation, or if they do, yield a fouling structure that provides little resistance to permeate flow (such as an open-structure aggregate with low fractal dimension). This is not to say that filter fouling does not occur in the tests shown in Figure 6.2; fouling does occur and is evidenced by flux decline. However, the fouling structure formed (be it pore blocking, constriction, or cake formation) does not provide a resistance to flow equal to that observed for the PEP simulant. While this difference could be attributed to particle concentration and suspending phase chemistry differences between the boehmite suspensions and the PEP simulant, the FeOOH filter flux results (shown in Figure 6.3 and discussed below) indicate that primary cause is likely particle chemistry. With respect to particle concentration, the permeate production rate of PEP simulant at solids concentrations below the transition to gel polarization at $\sim 20$-wt $\%$ UDS (Daniel et al. 2009b) is not expected to depend strongly on solids concentration. While solids concentration may impact the rate of fouling (in that it changes the mass of particles brought to the filter surface by flux), the impact on flux magnitude is expected to be small based on previous filtration tests where slurry concentration was varied.

With respect to backpulsed operations, both boehmite solids show a relatively consistent flux recovery after backpulsing. The recovered flux does not appear to decline significantly over the duration of 12-hour backpulsed operations (relative to that observed for the PEP simulant), which suggests that fouling of the filter by boehmite solids is mostly reversible. However, quantitative comparison of fluxes averaged over the initial hour of testing and over the first hour of the final 12-hour hold indicates an approximate $10 \%$ and 3\% irrecoverable flux loss for coarse boehmite and fine boehmite, respectively. For comparison, flux loss for the $\sim 3-\mathrm{vol} \%$ PEP simulant is typically around $25 \%$ during the initial period of filtration and immediately after the backpulse series at 100-hrs. First, it should be noted that the difference in irreversible flux loss is not a result of the different test scheme (100-hour test in Series 1 versus 36-hour backpulsed test in Series 2), as a 36-hour test format of the PEP simulant slurry on the 2 -foot CUF filtration system also showed a 25\% flux loss (see Daniel et al. 2009b). Thus, it is likely that the reduced loss of flux observed for the boehmite component results from lower solids concentration relative to the PEP simulant or from the difference in suspending phase chemistry. Indeed, as is discussed below, the only component that shows comparable irreversible flux loss to the PEP simulant is $\mathrm{CrOOH}$, which is not a baseline component in the PEP simulant.

Figure 6.3 shows the results of baseline component testing for iron oxy-hydroxide (FeOOH) solids (at the baseline ionic strength of $0.05 \mathrm{M} \mathrm{NaNO}_{3}$ ). The FeOOH suspension shows the decline in filter flux with time expected for waste simulant solids. However, the fluxes are about one order of magnitude lower than that observed for coarse boehmite solids and are typical of filter fluxes observed for PEP simulant suspensions at $\sim 3$-vol\% solids. Similarity of FeOOH suspension flux to that of PEP simulant suspensions indicates that $\mathrm{FeOOH}$ solids are the major driver of flux behavior in PEP simulants. In other words, $\mathrm{FeOOH}$ solids appear to be a significant fouling component. Examination of the $\mathrm{FeOOH}$ suspension performance during backpulsed operations suggests that flux decline over time is reversible; however, quantitative analysis of the filter flux both before and after backpulsed operations indicates a $\sim 10 \%$ irrecoverable loss of filter flux. This flux loss is lower than observed for the PEP simulant, and as postulated in the preceding paragraph, this difference likely results from the lower test concentration of the $\mathrm{FeOOH}$ component or from the difference in suspending phase chemistry between component baseline tests relative to the PEP simulant. 

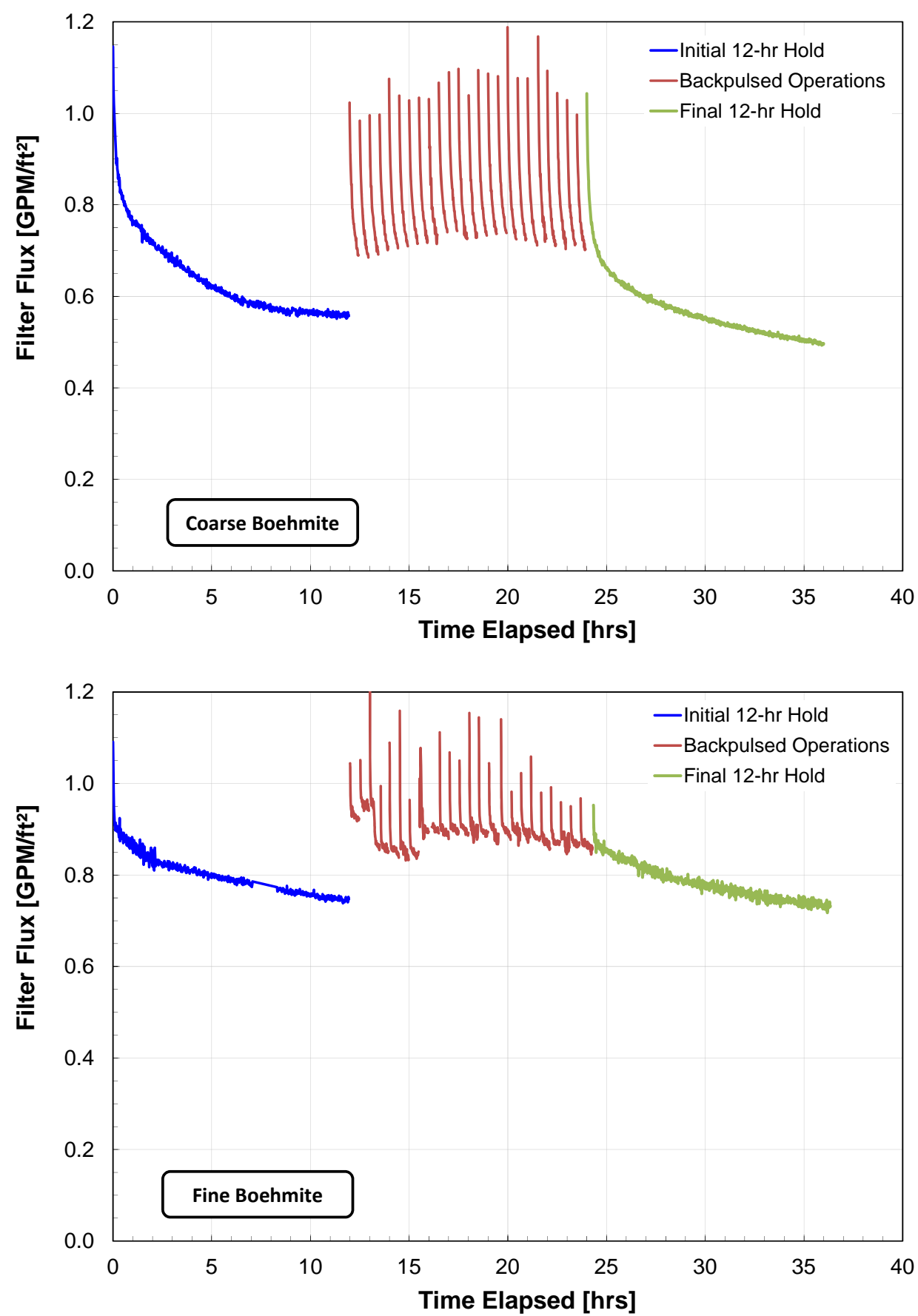

Figure 6.2. Results of Component Baseline Testing for Coarse and Fine Boehmite Solids. Both tests employ a $0.1-\mathrm{vol} \%$ suspension at $\mathrm{pH} 10$ and a background electrolyte concentration of $0.05 \mathrm{M} \mathrm{NaNO}_{3}$. 

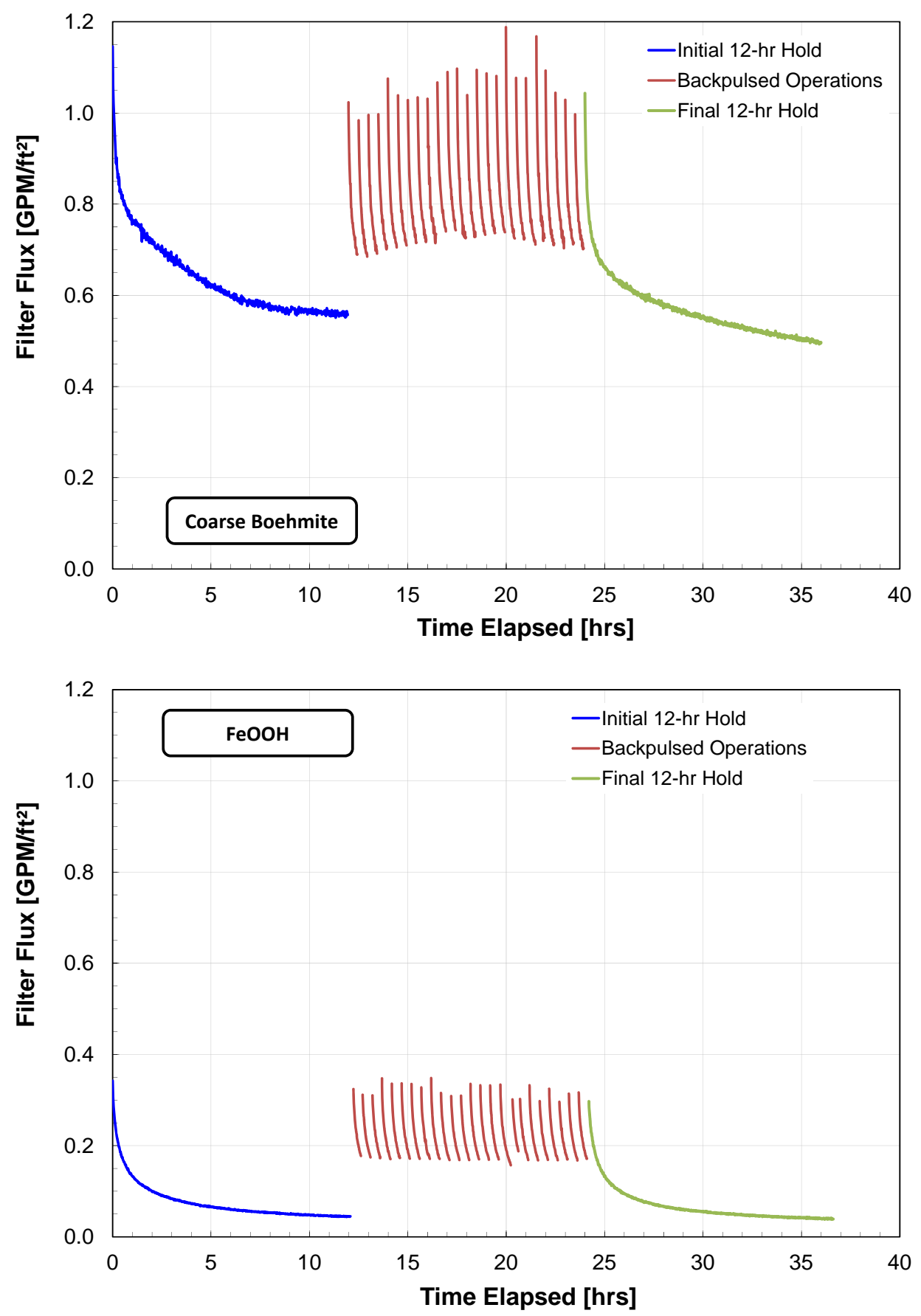

Figure 6.3. Comparison of Component Baseline Testing Results for Coarse Boehmite and Iron Oxy-hydroxide (FeOOH) Solids. Both tests employ a 0.1 -vol\% suspension at $\mathrm{pH} 10$ and a background electrolyte concentration of $0.05 \mathrm{M} \mathrm{NaNO}_{3}$. 
Figure 6.4 shows the results of baseline component testing for chrome oxy-hydroxide $(\mathrm{CrOOH})$ solids. The behavior of $\mathrm{CrOOH}$ solids is similar in terms of flux magnitude to $\mathrm{FeOOH}$ solids and suggests that $\mathrm{CrOOH}$ will also be a significant contributor to the filtration behavior of $\mathrm{CrOOH}$ bearing simulant slurries. This behavior is notable in that the majority of $\mathrm{CrOOH}$ particles by volume ( $\sim 92 \%)$ fall above the estimated pore size of $3 \mu \mathrm{m}$. This means that any fouling that occurs will likely be in the form of surface fouling or cake formation. Again, this highlights the importance of factors other than particle size, such as particle chemistry and particle-particle interactions, in filter fouling. The filter performance curves for $\mathrm{CrOOH}$ during the 12-hour continuous (non-backpulsed) segments are unusual in that both initial and final periods exhibit discontinuities in flux decline that cannot be associated with process changes in AV and TMP. Discontinuities occur as sudden increases in $\mathrm{CrOOH}$ permeate filter flux, and examples can be observed at 5, 26, and 27 hours of elapsed time in Figure 6.4. Such discontinuities are not observed during filtration of boehmite or $\mathrm{FeOOH}$ solids. While the cause for these sudden increases is unknown, it may involve disruption of a $\mathrm{CrOOH}$ filter cake given that the major of $\mathrm{CrOOH}$ solids cannot infiltrate the porous filter medium. A postulated scenario could be build-up of $\mathrm{CrOOH}$ up to and beyond a thickness that is only quasi-stable. A system perturbation such as a variation of axial velocity or mechanical vibration of pump may cause a cascade failure of the $\mathrm{CrOOH}$ cake structure that sheds a significant portion of the cake (yielding a rapid and sudden decrease in the resistance to filtration). Regardless of the cause, these discontinuities are of concern as they introduce uncertainty with respect to modeling the long-time fouling dynamics of $\mathrm{CrOOH}$ solids and simulants or actual waste solids that show similar behavior. With respect to backpulsing performance, examination of the filter performance for $\mathrm{CrOOH}$ during backpulsed operations suggests that flux decline over time is slightly irreversible. Quantitative analysis of the filter flux both before and after backpulsed operations indicates a $\sim 20 \%$ irrecoverable loss of filter flux. While this loss is comparable to that typically seen in the PEP simulant, $\mathrm{CrOOH}$ is not a baseline component of the PEP simulant.

Finally, Figure 6.5 compares the filtration behavior of $\mathrm{FeOOH}$ solids at two different background concentrations of $\mathrm{NaNO}_{3}(0.05$ and $0.1 \mathrm{M})$. This test illustrates the impact of ionic strength on filtration. FeOOH solids were selected because of the similarity of their filter flux magnitudes to the complete actual waste simulant. The results indicate that the change in ionic strength from $\sim 0.05 \mathrm{M}$ to $\sim 0.1 \mathrm{M}$ did not significantly impact filter flux behavior. The most significant change is observed in the variation of flux as a result of backpulsing. For tests in $0.05 \mathrm{M} \mathrm{NaNO}_{3}$, backpulsing yields a flux that varies from 0.17 to $0.33 \mathrm{GPM} / \mathrm{ft}^{2}$. In contrast, tests in $0.1 \mathrm{M} \mathrm{NaNO}_{3}$ show flux variation from 0.17 to only $0.25 \mathrm{GPM} / \mathrm{ft}^{2}$. This change suggests that in $0.1 \mathrm{M} \mathrm{NaNO}_{3}$, the initial reattachment of particles to the filter surface is more rapid than that in $0.05 \mathrm{M} \mathrm{NaNO}_{3}$, which would be consistent with reduction of the electric double layer around particles that could otherwise slow reattachment. It should be noted that this assertion is based on a single test observation and needs to be validated by additional testing. Despite the observed difference in backpulsed behaviors at different ionic strengths, the impact of changing ionic strength of long-term fouling behavior is minimal. Comparison of filter flux behaviors and magnitudes during periods of continuous operations shows little difference (if any) with changing ionic strength. For example, the flux at the end of testing in both cases is $\sim 0.04 \mathrm{GPM} / \mathrm{ft}^{2}$. Any observed difference is well within the repeatability between filter tests, such that when the curves are plotted together, they overlap one another. Thus, it can be concluded that while changing ionic strength may alter flux decline during the initial induction period of filtration, it does not impact longer time fouling behaviors (in terms of rate or flux magnitudes achieved). 

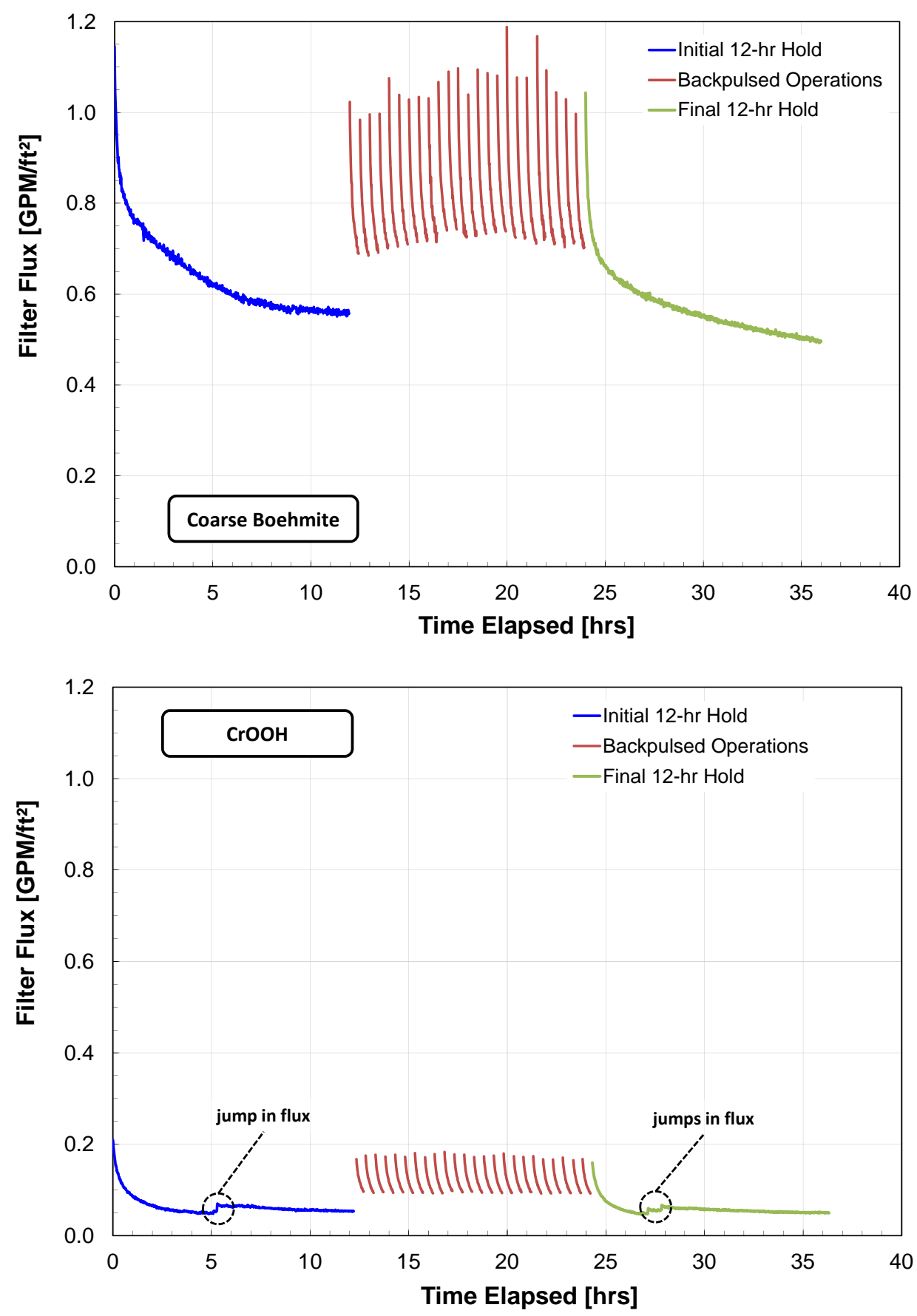

Figure 6.4. Comparison of Component Baseline Testing Results for Coarse Boehmite and Chrome Oxy-hydroxide $(\mathrm{CrOOH})$ Solids. Both tests employ a $0.1-\mathrm{vol} \%$ suspension at $\mathrm{pH} 10$ and a background electrolyte concentration of $0.05 \mathrm{M} \mathrm{NaNO}_{3}$. 

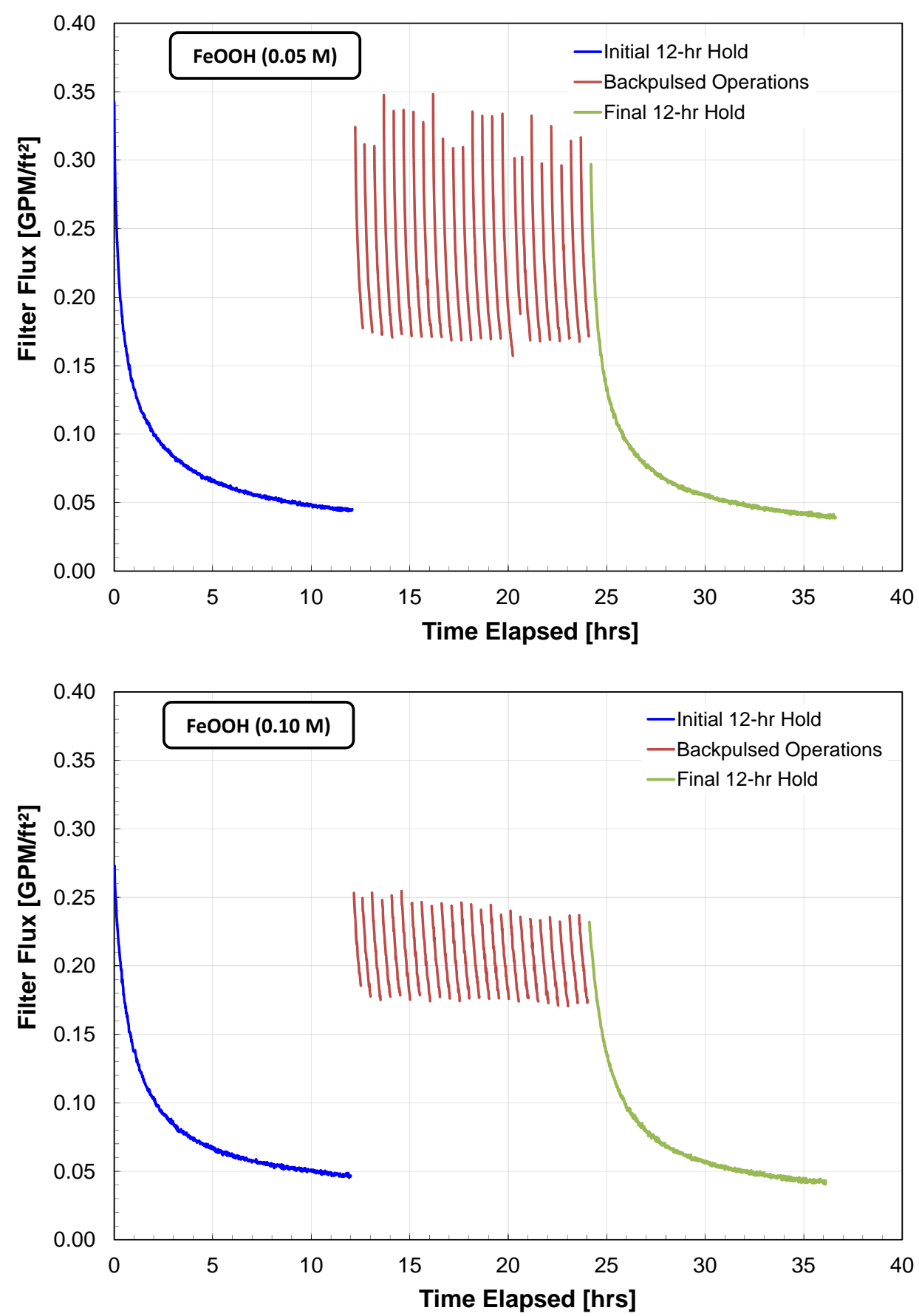

Figure 6.5. Comparison of FeOOH Filter Flux Behavior at Different Background Concentrations of $\mathrm{NaNO}_{3}$. Both tests employ a 0.1-vol\% suspension at $\mathrm{pH} 10$. 


\section{2 pH Dependence Testing}

Testing of the $\mathrm{pH}$ dependence for the baseline simulant components outlined above was conducted immediately following each 36-hour baseline test. All tests started with an approximately $\mathrm{pH} 10$ $0.1-\mathrm{vol} \%$ suspension of boehmite, $\mathrm{FeOOH}$, or $\mathrm{CrOOH}$ solids in a $0.05 \mathrm{M} \mathrm{NaNO}_{3}$ suspending phase. The flux behavior at $\mathrm{pH} 10$ was monitored for 1 hour, following which the filter was backpulsed and the flux monitored for another 1-hour period. Next, $\mathrm{pH}$ was reduced to $\mathrm{pH} 9$ by titrating with $0.01 \mathrm{M} \mathrm{HNO}_{3}$. Increases in slurry volume were counteracted by dewatering a volume of suspending phase equivalent to that added by titration. At $\mathrm{pH} 9$, the flux was again monitored for 1-hour, and at the end of that 1-hour period, the filter was backpulsed and monitored for another 1 hour period. This process of titrating, dewatering, monitoring, and backpulsing was repeated until a $\mathrm{pH}$ setpoint of 3 was reached and examined. For some tests, the $\mathrm{pH}$ was restored to $>7$ after $\mathrm{pH} 3$ testing was complete to examine the reversibility of any changes in the filter flux with $\mathrm{pH}$.

As with baseline component testing, all tests were run in continuous recycle filtration mode to maintain a constant concentration of $\sim 0.1-\mathrm{vol} \%$ solids with exception of dewatering to balance volume increases during titration of the suspension. Tests were run at an AV of $15 \mathrm{ft} / \mathrm{s}$ and a TMP of $40 \mathrm{psid}$ using a 2-foot tubular filter element.

Because $\mathrm{pH}$ testing is conducted under conditions where electrostatic effects could be important (i.e., relatively low ionic strength and a range of $\mathrm{pH}$ ), the IEP for each particle system was measured. This allows a rough assessment of particle-particle and particle-filter interactions. Table 6.3 shows the IEP for each particle system tested. Measurement of filter medium IEP can be done using streaming potential. Experimental studies that describe and employ a streaming potential to determine filter media zeta potential include Broz and Epstein (1976); Benavente and Fernandez-Pineda (1985); Nystrom et al. (1989); Martinez et al. (2002)' and Lawrence et al. (2006). It can also be measured using the electroviscous effect as described in Huisman et al. (1998). However, for the current study, these techniques were not available. Previous studies of 316-L stainless steel IEP in the peer-reviewed literature report a highly variable IEP that can range from $\mathrm{pH}$ of $\sim 5$ up to $\sim 9$, depending on filter history (Suzuki 2006).

Table 6.3. Isoelectric Points (IEPs) of Series 2 Test Components

\begin{tabular}{cc}
\hline Particle System & IEP \\
\hline Coarse Boehmite & 7.0 \\
Fine Boehmite & 9.5 \\
FeOOH & 7.9 \\
CrOOH & 7.6 \\
\hline
\end{tabular}

Figure 6.6 shows the filtration performance of coarse and fine boehmite suspensions at different $\mathrm{pH}$. Each shaded region in the plots corresponds to a different $\mathrm{pH}$ setpoint. The plot for coarse boehmite solids shows $\mathrm{pH}$ regions ranging from $\mathrm{pH} 10$ to $\mathrm{pH}$. The plot for fine boehmite solids shows this $\mathrm{pH}$ band as well, but also includes a final $\mathrm{pH}$ region from $\sim 21$ to 23 hours where the $\mathrm{pH}$ was restored to 9.5. The jump in filter flux in the middle of each $\mathrm{pH}$ band (i.e., the shaded regions) is caused by backpulsing of the filter. The results for $\mathrm{pH}$ testing show that the filtration behavior for coarse boehmite shows only minor dependence on $\mathrm{pH}$. This is expected, because the coarse boehmite is composed of relatively large particles that are not influenced by colloidal (e.g., electrostatic) forces and cannot access the interior of 

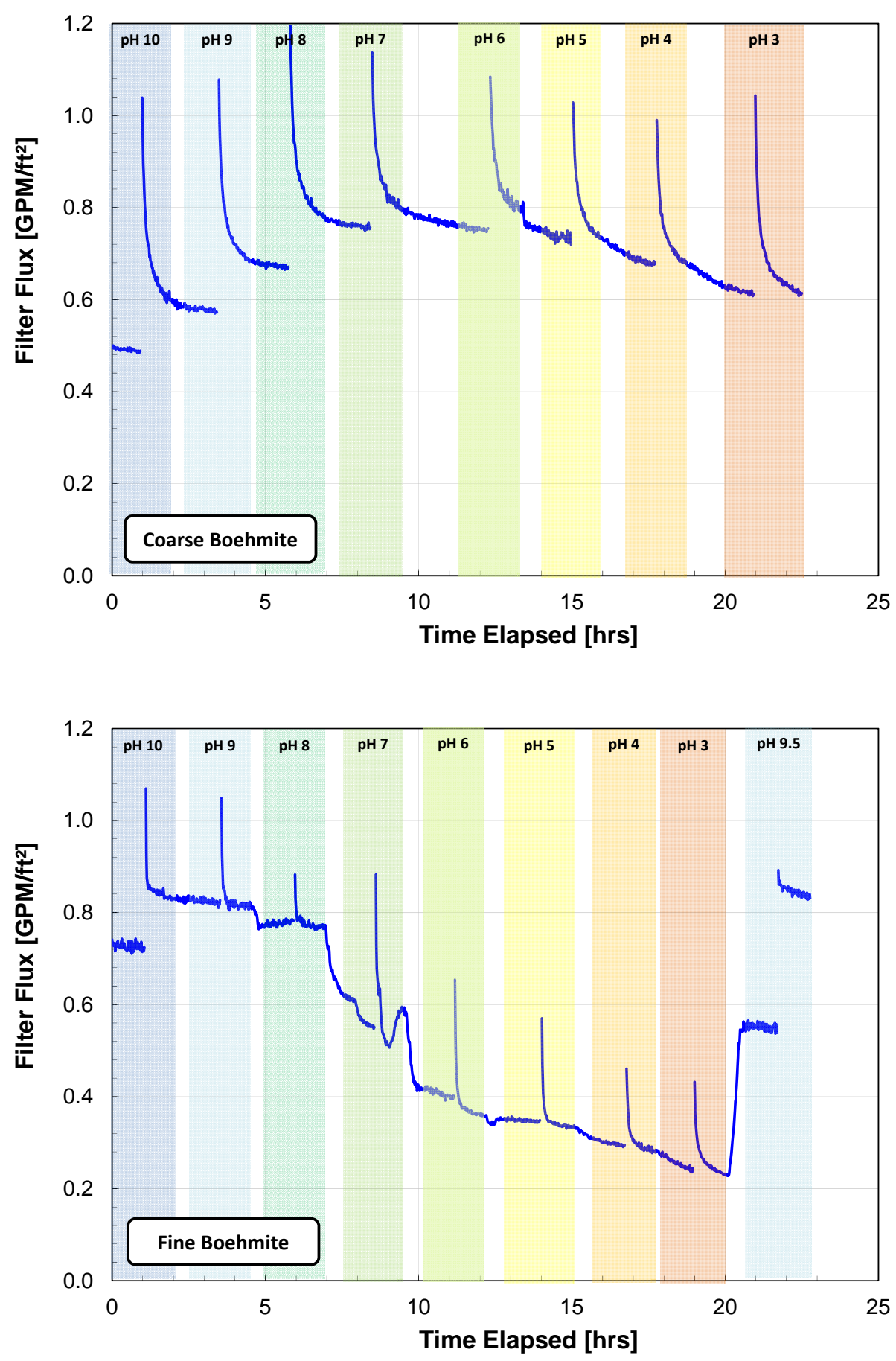

Figure 6.6. Comparison of Filter Flux for Coarse and Fine Boehmite Solids during pH Dependence Testing. Both tests employ a 0.1 -vol\% suspension in a $0.05 \mathrm{M} \mathrm{NaNO}_{3}$ suspending phase. Shaded regions indicate different $\mathrm{pH}$ setpoints. 

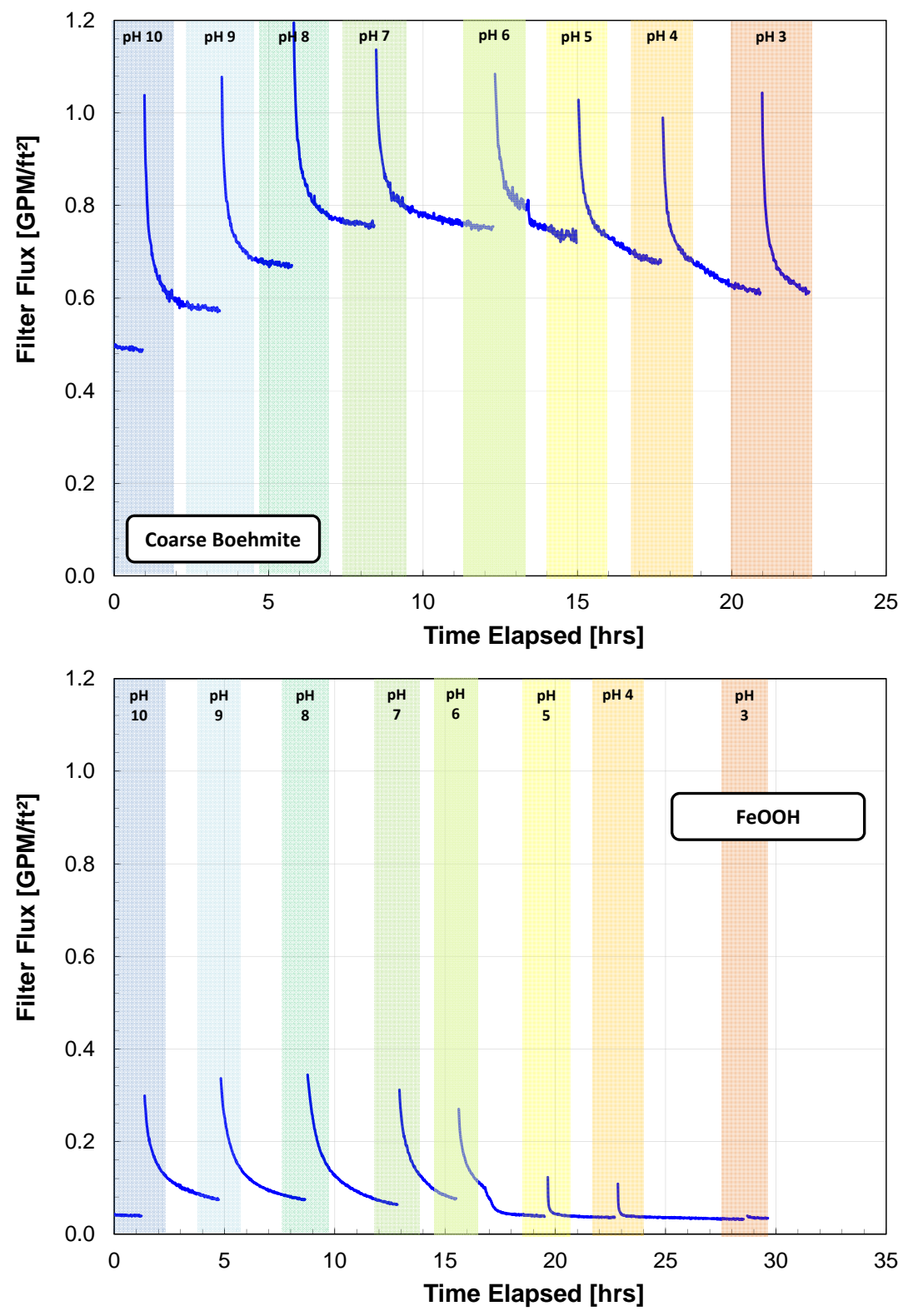

Figure 6.7. Comparison of Filter Flux for Coarse Boehmite and FeOOH Solids during pH Dependence Testing. Both tests employ a $0.1-\mathrm{vol} \%$ suspension in a $0.05 \mathrm{M} \mathrm{NaNO}_{3}$ suspending phase. Shaded regions indicate different $\mathrm{pH}$ setpoints.

the filter medium because of pore exclusion by size. In contrast, the filter behavior of fine boehmite suspensions was strongly dependent on $\mathrm{pH}$, and, as expected, based on size and pore access arguments, the fine boehmite suspension shows a region of strong fouling between $\mathrm{pH} 7$ and 3 , such that the flux is strongly reduced in this $\mathrm{pH}$ range. The reduction in filter flux observed during $\mathrm{pH}$ reduction can be reversed by restoring high $\mathrm{pH}$. An initial recovery is observed by reverse titration alone, and backpulsing at high $\mathrm{pH}$ appears to completely restore the initial flux. The data also indicate that while a decrease in $\mathrm{pH}$ appears to reduce flux, it does not appear to reduce the efficacy of backpulsing. Specifically, backpulsing appears to yield a significant (but temporary) increase in filter flux at all $\mathrm{pH}$ setpoints for both boehmite solids. 
Figure 6.7 shows the filtration performance of the $\mathrm{FeOOH}$ suspension at different $\mathrm{pH}$ (relative to that for coarse boehmite). The plot for $\mathrm{FeOOH}$ solids shows $\mathrm{pH}$ regions ranging from $\mathrm{pH} 10$ to $\mathrm{pH} 3$ and does not include a $\mathrm{pH}$ reversal. The results in Figure 6.7 indicate that the filter flux of FeOOH solids is reduced by $50 \%$ below a $\mathrm{pH}$ of 6 . This significant reduction in flux is also accompanied by a reduction in the efficiency of backpulsing. Specifically, backpulsing becomes progressively less effective at restoring flux below $\mathrm{pH}$. This change suggests a strong propensity for FeOOH solids to stick to and foul the filter at low $\mathrm{pH}$.

Figure 6.8 shows the filtration performance of the $\mathrm{CrOOH}$ suspension at different $\mathrm{pH}$ (relative to that for coarse boehmite). The plot for $\mathrm{CrOOH}$ solids shows $\mathrm{pH}$ regions ranging from $\mathrm{pH} 10$ to $\mathrm{pH} 3$ and includes a final reversal to $\mathrm{pH}$ 9. It should be noted that the $\mathrm{pH} 5$ setpoint was not tested for $\mathrm{CrOOH}$ solids. The behavior of $\mathrm{CrOOH}$ solids is similar to that for FeOOH solids. Below $\mathrm{pH} 7$, the rate of flux decline appears significantly increases, yielding lower filter flux relative to high $\mathrm{pH}$. Below $\mathrm{pH} 6$, backpulsing becomes progressively less effective at restoring flux. However, it appears that both loss of filter flux and backpulsing effectiveness can be restored by returning the suspending phase to high $\mathrm{pH}$.

Figure 6.9 shows the filtration performance of $\mathrm{FeOOH}$ over a range of $\mathrm{pH}$ with different background concentrations of $\mathrm{NaNO}_{3}(0.05$ and $0.1 \mathrm{M})$. The test results for $\mathrm{pH}$ testing of $\mathrm{FeOOH}$ with different ionic strength suspending phase mirror those for component testing. At the lower background concentration of $\mathrm{NaNO}_{3}$, backpulsing shows a larger dynamic range of flux immediately following the backpulse. After the induction period following backpulsing, the flux magnitude and rate of decline are similar for both 0.05 and $0.1 \mathrm{M} \mathrm{NaNO} 3$ test suspensions such that if plotted together, the curves at each $\mathrm{pH}$ setpoint would overlap. This result confirms the assertion made for component testing that changing ionic strength does not significantly impact long-time ( $>5$ minutes) fouling behavior of $\mathrm{FeOOH}$, even when testing over a broad range of $\mathrm{pH}$.

Overall, examination of the filter flux behaviors indicates changes in suspending phase chemistry appear to impact the filtration behavior of $\mathrm{FeOOH}, \mathrm{CrOOH}$, and fine boehmite. Filtration of coarse boehmite does not appear to be strongly impacted by changes in $\mathrm{pH}$ although there does appear to be some weak dependence. With respect to the role of surface charge, the results indicate that there are regions of strong fouling for several of the test suspensions. However, it is difficult to correlate these strong fouling regions to the IEP. Rather, it is noted that filter flux in $\mathrm{pH}$ regions near the IEP of the material are high relative to other $\mathrm{pH}$ ranges. As such, it appears that a flux maximum is associated with the IEP. While the manner in which this behavior is associated with particle and membrane charge is not investigated or considered in the current study, it highlights that there may be surface interactions associated with the IEP that can be used, by controlling $\mathrm{pH}$ or surface charge, to increase filter flux. Additional study of the current data and/or additional testing is recommended to better understand these mechanisms.

\subsection{Series 2 Test Conclusions}

Series 2 testing examined filtration behavior of individual components that were used in the PEP waste simulant slurry. The flux behavior of each component was characterized 1) during continuous and backpulsed operations as a function of time and 2) during titration of the dispersion from a $\mathrm{pH}$ of 10 to 3 . 

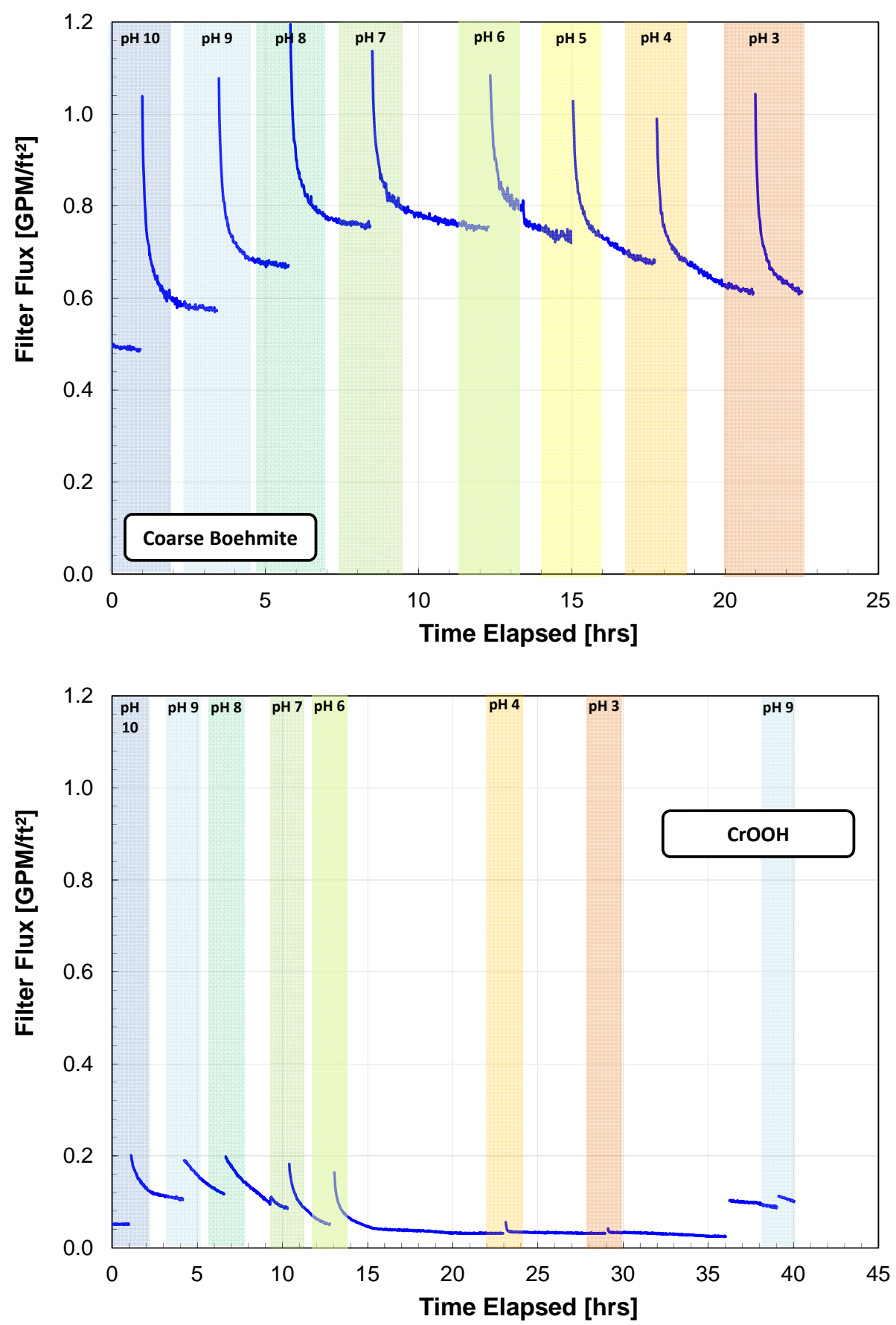

Figure 6.8. Comparison of Filter Flux for Coarse Boehmite and CrOOH Solids During pH Dependence Testing. Both tests employ a $0.1-\mathrm{vol} \%$ suspension in a $0.05 \mathrm{M} \mathrm{NaNO}_{3}$ suspending phase. Shaded regions indicate different $\mathrm{pH}$ setpoints. 

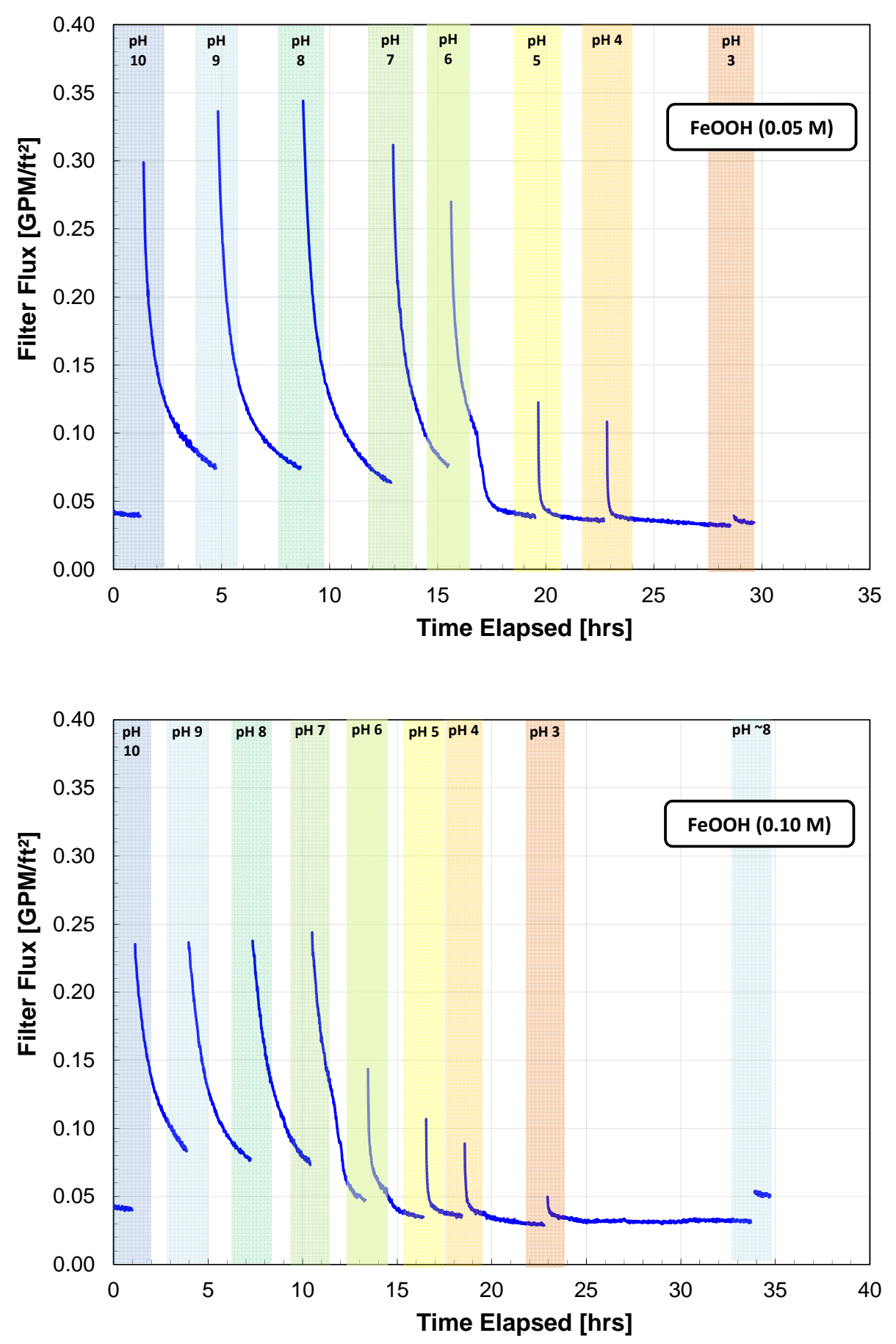

Figure 6.9. Comparison of Filter Flux for FeOOH Solids at Different Background Concentrations of $\mathrm{NaNO}_{3}$ during $\mathrm{pH}$ Dependence Testing. Both tests employ a $0.1-\mathrm{vol} \%$ suspension. Shaded regions indicate different $\mathrm{pH}$ setpoints. 
These two test approaches were termed component testing and $\mathrm{pH}$ dependency testing, respectively. A summary of results determined by these two test approaches used for Series 2 testing follows:

- Component baseline testing identified $\mathrm{FeOOH}$ and $\mathrm{CrOOH}$ solids as major fouling components that, even as 0.1 -vol\% suspensions, showed filter fluxes similar to $\sim 3$-vol\% PEP simulant slurries. In contrast, boehmite solids show filter fluxes that are one-order of magnitude greater than typically observed for PEP simulant sludges. All solids suspensions tests showed a significant degree of reversibility with respect to fouling, such that backpulsing was an effective means of maintaining high filter fluxes. While flux loss was completely reversible for boehmite solids, $\mathrm{FeOOH}$ and CrOOH solids did show a small 10-20\% irrecoverable loss in filter flux.

- $\mathrm{pH}$ dependence testing highlighted several interesting behaviors. As expected, the filter flux of suspensions containing fine particles shows a strong dependence on solution $\mathrm{pH}$ (relative to that observed for suspensions of coarse particles). In addition, regions of "strong" fouling were observed for fine boehmite, $\mathrm{FeOOH}$, and $\mathrm{CrOOH}$ suspensions, indicating that charge effects may be important for cross-flow filtration of the PEP simulant. For boehmite solids, backpulsing efficiency appears to be unaffected by changes in $\mathrm{pH}$ such that backpulsing always yielded a significant (but temporary) increase in flux. For $\mathrm{CrOOH}$ and $\mathrm{FeOOH}$ solids, backpulsing becomes progressively less effective as $\mathrm{pH}$ is decreased. Testing for $\mathrm{CrOOH}$ and fine boehmite solids indicates that returning the suspending phase to a high $\mathrm{pH}$ can restore lost flux and backpulse efficiency.

Overall, Series 2 testing to date has identified several key features of the filtration behavior with respect to the PEP filtration simulant. First, the tests provide strong evidence that iron oxy-hydroxide solids, which are a major component of the PEP simulant, drive the filtration behavior. Second, the test results show that factors other than particle size (in particular, suspending phase chemistry or particle chemistry) have a strong impact on filtration behavior. It should be noted that evidence of this was already identified in previous fouling studies by Russell et al. (2003), but Series 2 testing provides the first direct evidence for PEP simulant components on an individual basis.

As indicated above, the filter flux of PEP simulant components varied significantly. Figure 6.10 provides a summary of component fluxes measured during the first hour of continuous filtration before and after backpulsing. Both $\mathrm{CrOOH}$ and $\mathrm{FeOOH}$ dispersions at $0.1-\mathrm{vol} \%$ produced fluxes similar to the PEP simulant, while both boehmite solids tested produced fluxes about an order of magnitude greater than the PEP simulant. CrOOH solids showed the lowest flux, but also had the largest fraction of particles that were larger than the estimated pore size $(3 \mu \mathrm{m})$ of the filter medium. The results for $\mathrm{FeOOH}$ illustrate that an increase in background electrolyte from 0.05 to $0.1 \mathrm{M} \mathrm{NaNO}_{3}$ did not impact the flux magnitude (or rate of filter flux decline). For all components, the flux after backpulsed operations is less than the initial flux, indicating that fouling is irreversible to a slight extent. The baseline simulant components (i.e., boehmite and $\mathrm{FeOOH}$ ) show 3-10\% irrecoverable loss of flux, which is smaller than that observed for the PEP simulant $(\sim 25 \%)$. A review of Series 2 test data suggests that the difference is driven by the lower test concentration ( 0.1 -vol $\%$ for component testing versus $\sim 3 \%$-vol $\%$ for PEP simulant testing) or a change in the suspending phase chemistry. As such, the results for Series 2 testing suggest that the major drivers for filter flux behavior of the PEP simulant appear to be particle chemistry, slurry solids concentration, and/or suspending phase chemistry. The tests show that even at low solids concentration, a single component can drive the filtration behavior. 
$\mathrm{pH}$ dependence testing illustrates the importance of suspending phase chemistry on how slurry solids interact with the filter. For most components tested, particularly $\mathrm{FeOOH}$ and $\mathrm{CrOOH}$ solids, the results show that regions of strong fouling with respect to backpulsing can occur with changes in suspending phase chemistry. This illustrates understanding the filtration behavior over the entire filtration process, including post-leach, washing, and cleaning operations. The current tests showed poor backpulse efficiency at low $\mathrm{pH}$. The results may be important for acid cleaning of the filters, as they suggest that major simulant components tend to stick to the filter during low $\mathrm{pH}$ operations.

Overall, Series 2 testing of PEP simulant components has provided insight as to which components control the fouling behavior observed in testing of PEP simulant and as to which parameters (such as particle concentration) control the extent of reversible and irreversible filter fouling. The tests employed herein provide a useful means of not only characterizing filtration behavior but also provide insight into how testing of single components can be used to identify fouling components that can be added to mixtures of solids to produce the desired filtration behaviors.

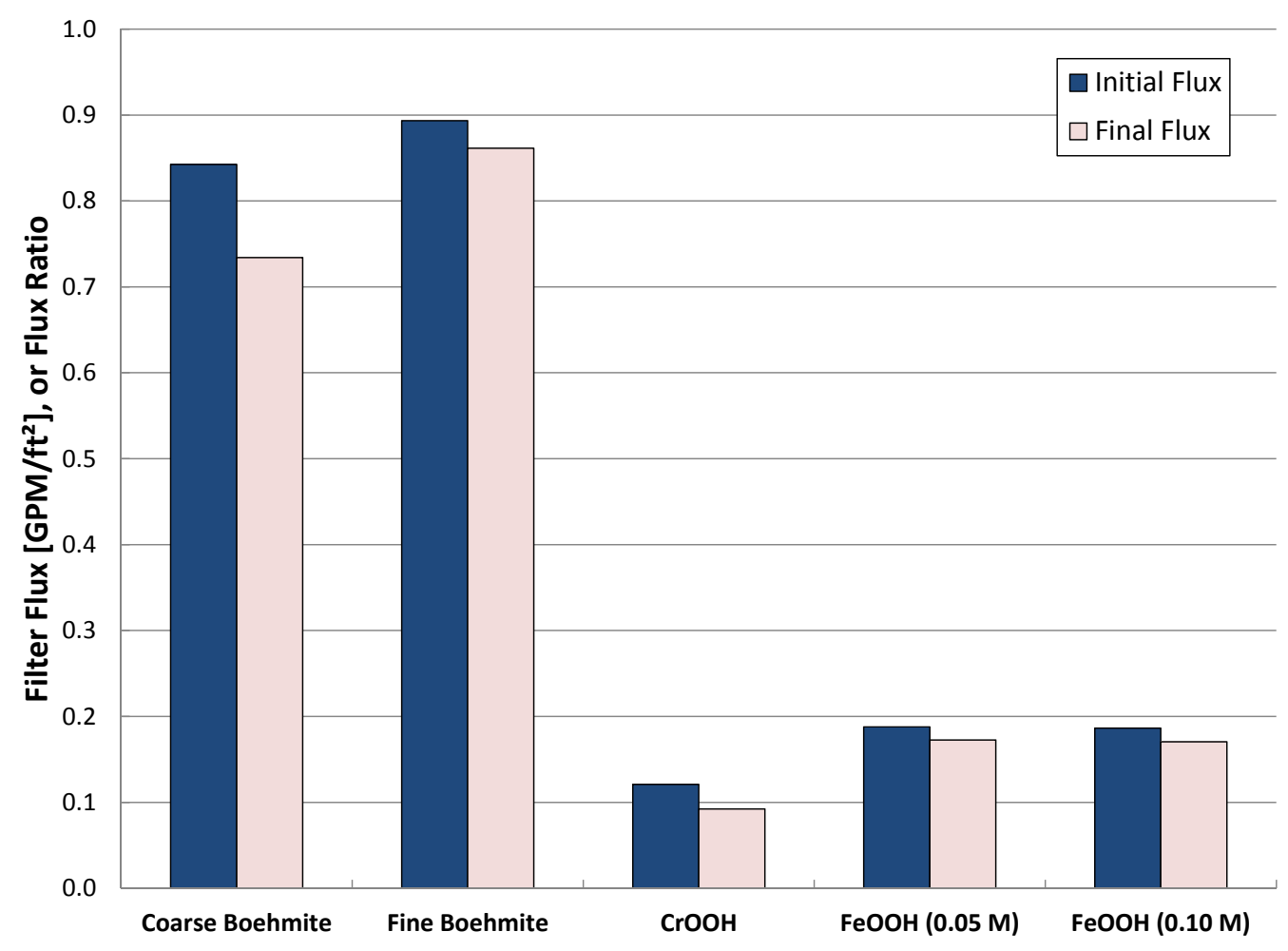

Figure 6.10. Summary of Component Testing Fluxes Measured During the Initial and Final 12-Hour Period of Continuous Filtration (flux for FeOOH solids shown at both 0.05 and $0.1 \mathrm{M} \mathrm{NaNO}_{3}$ ) 


\subsection{Series 3 Testing Results}

Series 3 focused on studies that evaluated the efficacy of chemical cleaning operations in restoring filter performance. These studies provide insight with regard to the relative and overall effectiveness of different chemical cleaning operations and to what range of "clean" filter flux should be expected from chemical cleaning. To this end, three series of cleaning tests were performed: 1) one series incorporated into Series 1 filtration testing, 2) another series incorporated into Series 2 filtration testing, and 3) a final independent series that employed a $\mathrm{SrCO}_{3}$ test slurry. In the following sections, the results of each set of cleaning tests are introduced and discussed in detail.

\subsection{Estimates of Clean Filter Flux}

As discussed in Section 4.1.1, the filter elements were purchased from Mott and are 316-L sintered stainless steel elements with a Mott Grade of 0.1 . While the equipment section provides key information on the dimensions of the filters used in the CUF filtration apparatus, it provides little information on baseline performance expected from these filters. In the current section, analysis of manufacturer data, specifically isopropanol filter flux data from Rubow and Jha (1999), will be used to derive a baseline ("clean") filter flux for the Mott filter element used in the current study. These data will then be used to compare against clean water flux measurements presented for Series 1, Series 2, and $\mathrm{SrCO}_{3}$ cleaning tests to assess the overall effectiveness of cleaning.

Rubow and Jha (1999) provide isopropanol filter flux data for a 316-L sintered stainless steel filter element with a thickness of 0.055 inches and a porosity of 0.25 . These data are reproduced in Table 7.1. The CUF filter elements used for the current studies are expected to have similar porosity to that used in Rubow and Jha (1999); however, they have a larger filter thickness of 0.0625 inches. As such, analysis of the filter flux data must take this difference into account. The flux behavior of a pure liquid is expected to follow Darcy's law, such that

$$
J=\frac{\Delta P}{\mu R_{m}^{o}}
$$

where $J$ is filter flux, $\Delta P$ is transmembrane pressure, $\mu$ is the fluid viscosity, and $R_{m}{ }^{o}$ is the baseline (clean) filter resistance. For isopropanol at $25^{\circ} \mathrm{C}$, the viscosity is $1.96 \mathrm{mPa} \mathrm{s}$ (Yaws 1999). The filter medium resistance can be expressed in terms of a resistance per unit length, $M_{m}{ }^{o}$, where

$$
R_{m}^{o}=M_{m}^{o} \mathrm{~L}
$$

where $L$ is the thickness of the filter medium. Thus,

$$
J=\frac{\Delta P}{\mu M_{m}^{o} L}
$$

For the current analysis, $M_{m}{ }^{o}$ is assumed to be unique for the sintered stainless steel filter from Mott. The value of $M_{m}{ }^{\circ}$ can be determined by linear regression analysis of the manufacturer's filter flux data in Table 7.1. The results of this analysis (presented in the first half of Table 7.2) indicate a unit length resistance of $1.36 \times 10^{14} \mathrm{~m}^{-2}$ for the stainless steel filter element. This result can then be applied to the calculation of baseline flux for CUF filtration processes. Using the known CUF filter element thickness 
of 0.0625 inches, two baseline fluxes were calculated: one for water and one PEP simulant supernate (which have viscosities of 0.89 and $2.80 \mathrm{mPa} \mathrm{s}$ at $25^{\circ} \mathrm{C}$, respectively). The baseline flux for water describes the best achievable CWF obtainable on the CUF filtration system at a given TMP (and at $25^{\circ} \mathrm{C}$ ) based on manufacturer's data. Likewise, the baseline flux for PEP simulant supernate describes the highest flux achievable for the PEP simulant at a given TMP (as it represents the case where no solid foul the filter). For these calculations, a baseline TMP of 40 psid is assumed.

Table 7.1. Isopropanol Flux Data from Rubow and Jha (1999) for a 316-L Stainless Steel Filter with a Thickness of 0.055 Inches

\begin{tabular}{cc}
\hline $\begin{array}{c}\Delta \mathrm{P} \\
{[\mathrm{psi}]}\end{array}$ & $\begin{array}{c}\text { Flux } \\
{\left[\mathrm{GPM} / \mathrm{ft}^{2}\right]}\end{array}$ \\
\hline 5 & 0.11 \\
10 & 0.27 \\
15 & 0.40 \\
20 & 0.53 \\
25 & 0.69 \\
30 & 0.83 \\
\hline
\end{tabular}

Table 7.2. Results of Regression Analysis of Flux Data and Calculation of Baseline CUF Filtration Rates for Clean Water and PEP Simulant Supernate

\begin{tabular}{|c|c|}
\hline Parameter & Value \\
\hline \multicolumn{2}{|c|}{ Analysis of Manufacturer's Data (Rubow and Jha 1999) } \\
\hline Filter thickness, inches & 0.055 \\
\hline Filter performance data & Isopropanol flux - Table 7.1 \\
\hline Fluid viscosity $\left(25^{\circ} \mathrm{C}\right), \mathrm{mPa} \mathrm{s}$ & 1.96 \\
\hline Regressed filter resistance, ${ }^{(a)} \mathrm{m}^{-1}$ & $1.90 \times 10^{11}$ \\
\hline Unit length resistance, $\mathrm{m}^{-2}$ (Eq. 7.2) & $1.36 \times 10^{14}$ \\
\hline \multicolumn{2}{|c|}{ Analysis of CUF Flux } \\
\hline Filter thickness, inches: & 0.0625 \\
\hline Filter resistance, $\mathrm{m}^{-1}$ (Eq. 7.2) & $2.16 \times 10^{11}$ \\
\hline \multicolumn{2}{|l|}{ Fluid viscosity, mPa s } \\
\hline Water $\left(25^{\circ} \mathrm{C}\right)$ & 0.89 \\
\hline PEP simulant supernate & 2.80 \\
\hline \multicolumn{2}{|c|}{ Calculated clean flux, GPM ft ${ }^{-2}$ (Eq. 7.1) } \\
\hline Water $\left(25^{\circ} \mathrm{C}\right)$ & 2.1 \\
\hline PEP simulant supernate & 0.67 \\
\hline
\end{tabular}

The results of baseline flux calculation for water and PEP simulant supernate are presented in the second half of Table 7.2. For water, the maximum achievable flux at $40 \mathrm{psid}$ is $2.1 \mathrm{GPM} / \mathrm{ft}^{2}$, which corresponds to a permeate rate of $2100 \mathrm{~mL} / \mathrm{min}$ on the 2-foot filter element. Likewise, the maximum flux expected for a PEP simulant is $0.67 \mathrm{GPM} / \mathrm{ft}^{2}$, which corresponds to a permeate rate of $670 \mathrm{~mL} / \mathrm{min}$ on the 2 -foot filter element. These estimates of maximum filter flux should be approached with some caution 
because they are based on manufacturer's data, and lot-to-lot variation of the filter element (such as variation in porosity) is to be expected such that the current lot of filter elements used for PEP and EM31 testing may show differences in actual filter performance. For clean water tests presented, the actual maximum flux achieved is found to be $\sim 2.5 \mathrm{GPM} / \mathrm{ft}^{2}$ (see Figure 7.7 ) and is slightly larger than that estimated. For PEP simulant, the actual flux for simulant slurries is much lower than the estimated flux because of the presence of solids that foul the filter and reduce flux. Despite these limitations, the baseline fluxes provided in Table 7.2 provide a useful baseline by which the flux behavior of cleaned filter elements may be judged.

\subsection{Series 1 Cleaning Test Results}

Cleaning tests associated with Series 1 studied the effectiveness of cleaning regimens after long-duration (100 hours) filter tests that used a 5-wt\% PEP simulant slurry. The CWF tests results associated with Series 1 cleaning activities (see Section 3.3.1) are presented in Figure 7.1. The results are presented on an adjusted time axis that allows presentation of three CWF tests in a single graph without needing to include the periods of actual simulant testing that occurred between them. As such, Figure 7.1 presents CWF data before each Series 1 test (i.e., pre-test), after each Series 1 test (but before acid cleaning), and after the oxalic cleaning process associated with each test. Within each CWF test segment are three sub-segments that correspond to CWF filtration at three different TMPs. All data are corrected to a single TMP of 40 psid for ease of comparison. The breaks between the sub-segments correspond to backpulsing of the filter element.

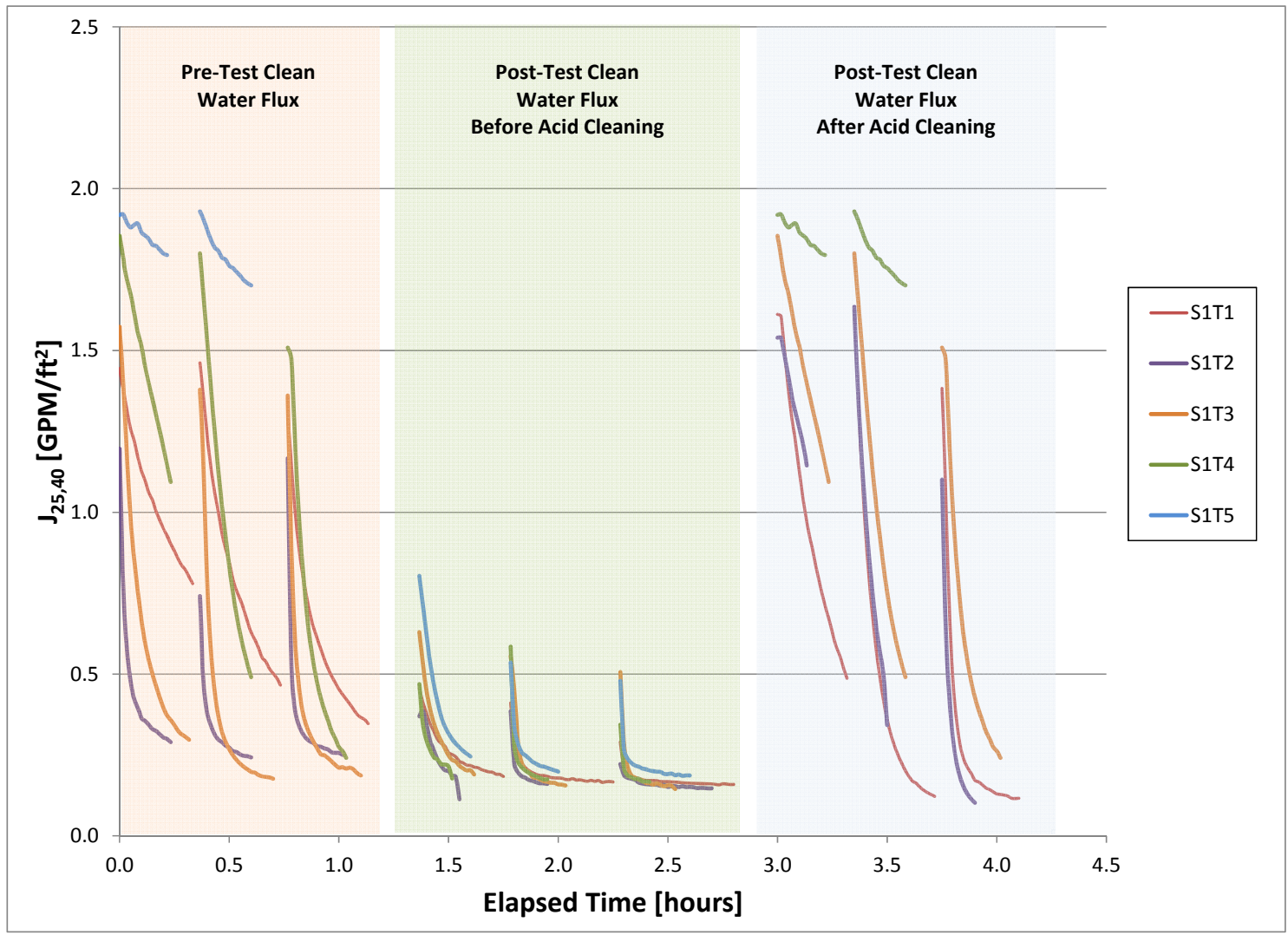

Figure 7.1. Clean Water Flux for Series 1 Tests 1-5 (corrected to a TMP of 40 psid). The time axis for flux data has been adjusted for the purpose of comparison. 
The initial pre-test CWF typically starts between 1.2 and $1.8 \mathrm{GPM} / \mathrm{ft}^{2}$, which falls somewhat below the maximum expected CWF flux results presented in Section 7.1. This is expected, given the expected lot-to-lot variation in filter elements and the fact that the filter employed in Series 1 tests has been used in previous filtration testing (see Section 4.1.1). In all cases, the pre-test CWF values rapidly decline. The rate of decline varies from test-to-test, and there is a broad range of CWF values at the end of the pre-test period of measurement (ranging from 0.2 to $1.7 \mathrm{GPM} / \mathrm{ft}^{2}$ ).

The rapid decay is consistent with fouling of the filter by particulate solids. Given that the test fluid (clean water) should be free of particulate matter, the origin of the particulate solids is likely from hold-up of particulate solids within the filter system. Areas for the greatest potential hold-up are those isolated from slurry flow, including the slurry reservoir (where shear flow is low relative to the circulation loop) and pressure sensor installation points (which have cavities receded from flow where solids can collect). It is plausible that some solids remain even after multiple rinses and prolonged exposure to acids during the cleaning regimens. It is postulated that during clean water testing, these trace solids detach and foul the filter element. While the exact concentration of trace solids required to cause the declines shown in Figure 7.1 is unknown, Series 2 testing (Section 6) has shown that low-volume concentration suspensions can effect large reductions in filter flux. Backpulsing during pre-test CWF measurements indicates that the fouling observed is largely reversible, as the flux recovered on backpulsing is similar to (but only slightly less than) that observed in the first of the three sub-segments.

The post-test (but before acid cleaning) fluxes are shown in the middle section of Figure 7.1. As expected, the post-test (before acid cleaning) CWF tests show lower overall fluxes. This expectation is based the fact that rinsing and backpulsing alone will not remove solids entrained on the filter. Filter flux observed in the post-test flux measurements before acid cleaning falls between 0.2 and $0.7 \mathrm{GPM} / \mathrm{ft}^{2}$. All fluxes show an initially high $\left(0.5-0.7 \mathrm{GPM} / \mathrm{ft}^{2}\right)$ flux followed by a rapid decline to $\sim 0.2 \mathrm{GPM} / \mathrm{ft}^{2}$. In general, these post-test CWF measurements show much better test-to-test agreement relative to the pre-test CWF data. This is also expected, given that the post-test filter has been conditioned (fouled) by the same PEP simulant slurry.

Finally, the post-test, after-acid cleaning CWF measurements are shown in the right-side of Figure 7.1. For a given Series 1 test, these flux data correspond to pre-flux data for the following test (such that the post-test after cleaning data for S1T1 correspond to the pre-test flux data for S1T2, and so on). In most cases, separate pre-test and post-acid cleaning CWF measurements were performed. The exceptions are S1T4 and S1T5, where the pre-test data is the same as the post-acid cleaning data from S1T3 and S1T4, respectively. S1T5 did not have a post-acid cleaning CWF measurement as the filter was not acid cleaned but was submitted for destructive analysis after rinsing with $0.01 \mathrm{M} \mathrm{NaOH}$ only. These results show that while acid cleaning is effective at restoring filter flux to pre-test levels of greater than $1 \mathrm{GPM} / \mathrm{ft}^{2}$, the filter flux decay rapidly to levels well below $1 \mathrm{GPM} / \mathrm{ft}^{2}$. As discussed above, the cause for this decline is trace solids not removed from the system during rinsing or by acid cleaning.

Overall, Series 1 CWF testing results shown in Figure 7.1 provide only a partial assessment of acid cleaning effectiveness. It can be stated that rinsing alone would not be considered effective in that it yields CWF that fall below the historic benchmark of $1 \mathrm{GPM} / \mathrm{ft}^{2}$ (see Section 1.3). While acid cleaning yields initial CWF above the $1 \mathrm{GPM} / \mathrm{ft}^{2}$ benchmark, which would be historically interpreted as having effectively cleaned the filter, this flux is not sustainable and rapidly declines to fluxes well below $1 \mathrm{GPM} / \mathrm{ft}^{2}$ in several of the test cases as a result of suspected trace particulate contamination. Since trace 
contamination impacts bench-scale CWF testing, where the system can be easily disassembled to access potential hold-up volumes, it can be expected to impact plant-scale operations where hold-up volumes cannot be easily accessed during cleaning.

For these reasons, it may be argued that the filter cleanliness can be best quantified by the clean water flux at an interval immediately following a backpulse. For the purposes of specifying the CWF for a given test condition, the average flux during the first 2 minutes of testing following a backpulse is used. Average flux for the pre-test CWF conditions and a comparison to the initial permeate flow for each of the Series 1 tests are given in Table 7.3. Even adjusted for viscosity ( $0.9 \mathrm{mPa}$ s versus $2.8 \mathrm{mPa} \mathrm{s})$, the pre-test $C W F$ values are $5 \times$ to $10 \times$ greater than the starting fluxes for simulant tests. The historical assumption has been that the low residual starting membrane resistance measured during CWF testing does not significantly bias the starting flux for simulant tests. To test this assumption quantitatively, an approach has been developed to correlate measured, average CWF values with average starting flux values for the Series 1 tests.

Table 7.3. Comparison of Initial Permeate Flux with Pre-test CWF for Each of the Series 1 Tests

\begin{tabular}{lcccc}
\hline Test & $\begin{array}{c}\text { Target } \\
\text { TMP } \\
{[\mathrm{psid}]}\end{array}$ & $\begin{array}{c}\text { Target } \\
\text { Axial } \\
\text { Velocity } \\
{[\mathrm{ft} / \mathrm{sec}]}\end{array}$ & $\begin{array}{c}\text { Initial Permeate } \\
\text { Flux }(\mathrm{t}=0 \rightarrow 2 \mathrm{~min}) \\
{\left[\mathrm{GPM} / \mathrm{ft}^{2}\right]}\end{array}$ & $\begin{array}{c}\text { Pre-Test CWF }(\mathrm{t}=0 \rightarrow 2 \mathrm{~min}, \\
\text { corrected to TMP }=40 \mathrm{psid}) \\
{\left[\mathrm{GPM} / \mathrm{ft}^{2}\right]}\end{array}$ \\
\hline S1T1 & 40 & 15 & 0.076 & 1.346 \\
S1T2 & 40 & 17 & 0.080 & 0.810 \\
S1T3 & 40 & 13 & 0.063 & 1.223 \\
S1T4 & 60 & 15 & 0.086 & 1.633 \\
S1T5 & 20 & 15 & 0.057 & 1.918 \\
\hline
\end{tabular}

An assessment of the effect of a measured CWF on the initial permeate flux can be made using the membrane resistance term in Darcy's Law. Since membrane resistances may be added in series, the total initial membrane resistance $\left(R_{t o t}\right)$ may be expressed as the sum of the resistance associated with the simulant $\left(R_{\text {sim }}\right)$ and a residual membrane resistance $\left(R_{\text {res }}\right)$ calculated from the CWF:

$$
J_{C W F}=\frac{\Delta P}{\mu R_{t o t}}
$$

where

$$
R_{\text {tot }}=R_{\text {sim }}+R_{\text {res }}
$$

Since the flux is inversely proportional to the membrane resistance, a percentage increase in membrane resistance results in the same percentage decrease in flux. Therefore, the percentage contribution of $R_{\text {res }}$ to $R_{\text {tot }}$ is a clear way of expressing the quantitative impact of the residual resistance after cleaning on initial filtration performance.

$$
\% \text { Contribution of } R_{\text {res }}=\frac{R_{\text {res }}}{R_{\text {tot }}} \times 100
$$


$R_{\text {tot }}$ may be calculated using the average permeate flux over a definite time interval at the start of filtration testing. $R_{\text {res }}$ must be defined: it is the difference between the total membrane resistance calculated during a definite interval of CWF testing $\left(R_{C W F}\right)$ and the membrane resistance of an ideally clean filter $\left(R_{\text {ideal }}\right)$ of the same type:

$$
R_{\text {res }}=R_{C W F}-R_{\text {ideal }}
$$

Several methods could be employed to determine $R_{\text {ideal }}$, but based on historical experience with these types of filter elements, the highest sustained values of CWF, corrected to a TMP of 40 psid have been near $2.5 \mathrm{GPM} / \mathrm{ft}^{2}$ (see Figure 7.7). For the purposes of this discussion, the $R_{\text {ideal }}$ will be calculated from a CWF value of $2.5 \mathrm{GPM} / \mathrm{ft}^{2}$. It should be noted that $R_{\text {ideal }}$ is equivalent to the term $R_{m}{ }^{o}$ in Section 7.1 ; the $R_{\text {ideal }}$ notation is employed to differentiate it from the estimate of "ideal" (clean) filter resistance based on manufacturer's data.

If $R_{\text {tot }}, R_{\text {sim }}$, and $R_{\text {ideal }}$ are fixed, it is possible to calculate the percentage contribution of $R_{\text {res }}$ to $R_{\text {tot }}$ as a function of $R_{C W F}$, and by extension, initial permeate flux. Such an exercise was performed for the Series 1 tests with the assumptions in Table 7.4 and a plot of the results in Figure 7.2. Calculated values of $R_{\text {res }}$ and $\mathrm{R}_{\text {tot }}$ and the ratio between them for each of the Series 1 cleaning tests are given in Figure 7.3. Based on the pure theoretical construct of $\mathrm{R}_{\text {res }}$, one might expect $\mathrm{S} 1 \mathrm{~T} 2$ to start at a relatively higher flux than $\mathrm{S} 1 \mathrm{~T} 1$, and expect S1T3 to start at a relatively lower flux than S1T1. However, the opposite is true in both cases, indicating that the $\mathrm{R}_{\text {res }}$ measure may be easily confounded by complex turbulent flow slurry dynamics prior to and shortly after the start of testing.

Table 7.4. Assumptions for Calculating the Percentage Contribution of $\mathrm{R}_{\mathrm{res}}$ to $\mathrm{R}_{\mathrm{tot}}$ as a Function of Clean Water Flux for Series 1 Tests

\begin{tabular}{llll}
\hline \multicolumn{1}{c}{ Variable } & \multicolumn{1}{c}{ Assumption } & Value & Units \\
\hline $\mathrm{R}_{\mathrm{ideal}}$ & $\begin{array}{l}\text { Calculated from a CWF value of } \\
2.5 \mathrm{GPM} / \mathrm{ft}^{2}\end{array}$ & $1.62 \mathrm{E} 11$ & $\mathrm{~m}^{-1}$ \\
$\mathrm{R}_{\mathrm{tot}}$ & $\begin{array}{l}\text { Calculated from a global average of the } \\
\text { first 2 minutes of flux data from each of } \\
\text { the five tests, 95\% confidence interval } \\
\text { applied }\end{array}$ & $1.96 \mathrm{E} 12$ & $\mathrm{~m}^{-1}$ \\
$\mathrm{R}_{\mathrm{tot}} 95 \% \mathrm{CI}$ & $\begin{array}{l}\text { The } 95 \% \text { confidence interval for the } \\
\text { global average of } \mathrm{R}_{\text {tot }}\end{array}$ & $4.22 \mathrm{E} 11$ & $\mathrm{~m}^{-1}$ \\
$\mathrm{R}_{\mathrm{CWF}}$ & $\begin{array}{l}\text { Variable, calculated from CWF values of } \\
0.1 \text { to } 2.5 \mathrm{GPM} / \mathrm{ft}^{2}\end{array}$ & 4.06 E12 to $1.62 \mathrm{E} 11$ & $\mathrm{~m}^{-1}$ \\
\hline
\end{tabular}




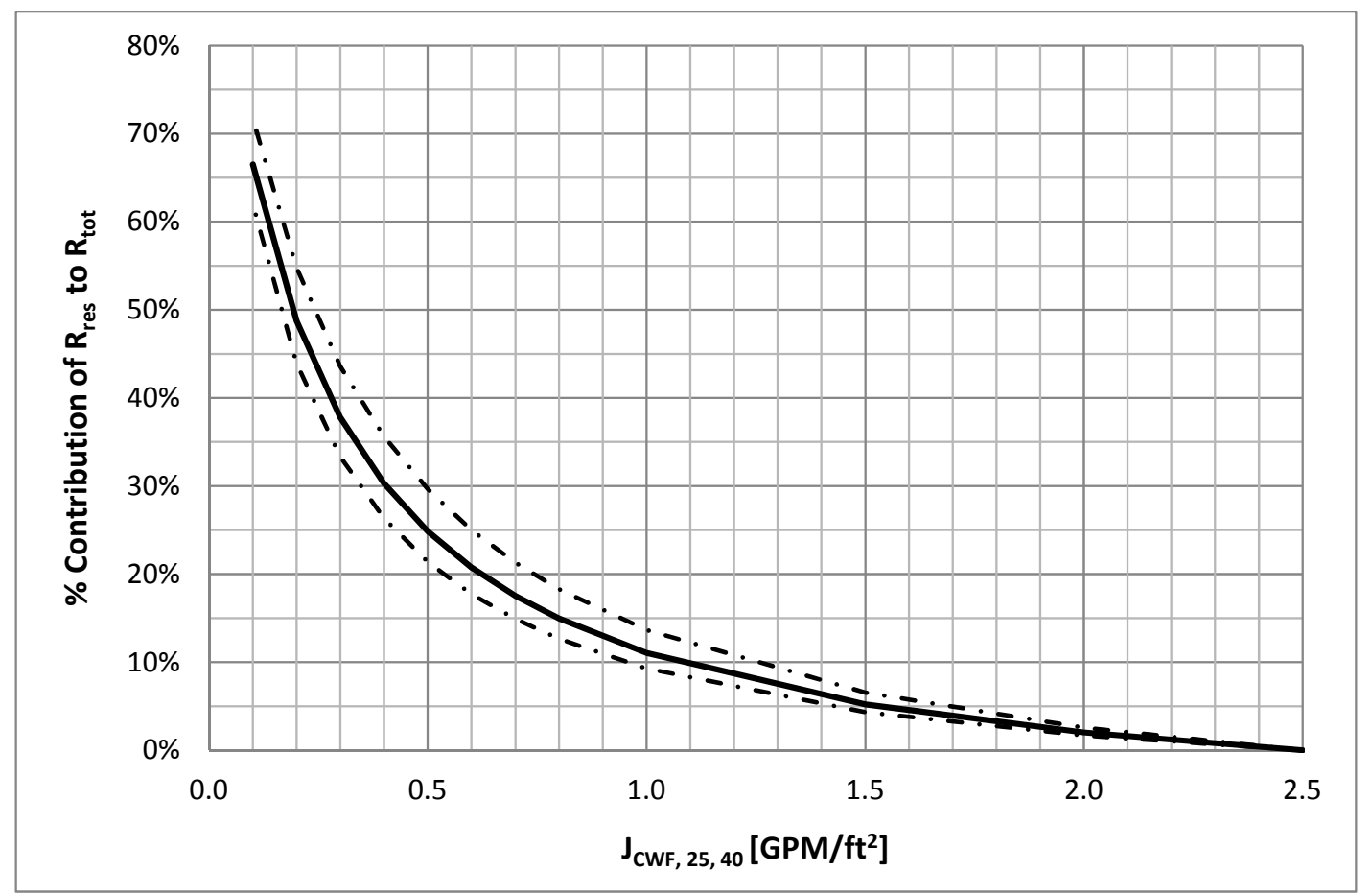

Figure 7.2. Calculated Percentage Contribution of $\mathrm{R}_{\text {res }}$ to $\mathrm{R}_{\text {tot }}$ as a Function of Clean Water Flux for Series 1 Testing Based on the Assumptions in Table 7.4

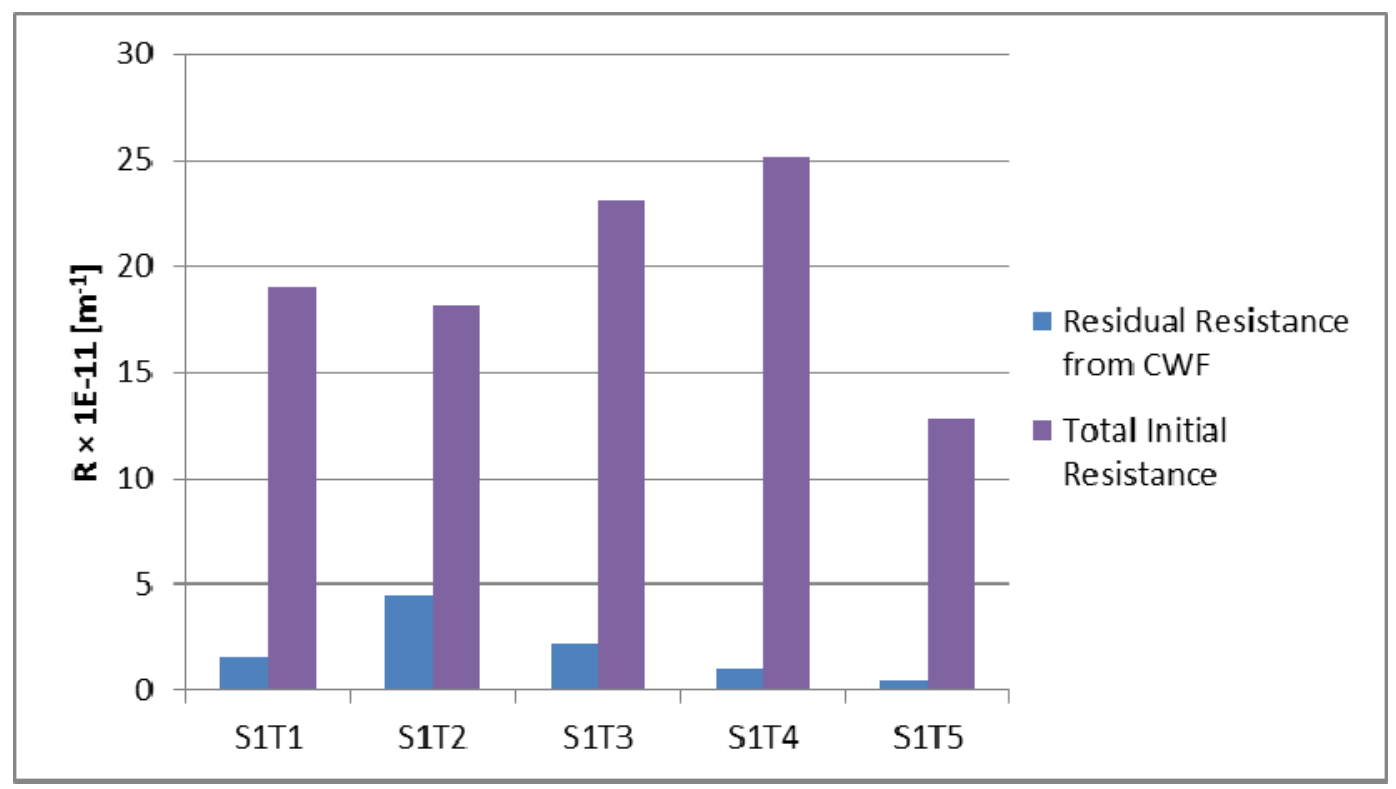

Figure 7.3. Calculated $R_{\text {res }}$ to $R_{\text {tot }}$ Values for Series 1 Testing Based on the Assumptions in Table 7.4

If $R_{\text {sim }}$ at the start of filtration was constant, as might be expected for sequential batches of the same simulant at similar operating conditions such as S1T1, S1T2, and S1T3, then the impact of $R_{\text {res }}$ to $R_{\text {tot }}$ should be obvious and scale with the magnitude of $R_{\text {res. }}$. The fact that the impact of $R_{\text {res }}$ cannot be seen reliably means that $R_{\text {sim }}$ is not constant. There are several parameters incorporated into $R_{\text {sim }}$ at 2 minutes into the test condition: 
- slurry mixing time in the system prior to the start of filtration operations

- slurry mixing conditions prior to the start of filtration

- time required to set target operating conditions

- adjustments to operating conditions in the first few minutes of operation

- permeate flux during the first few minutes of operation

- selection of $\mathrm{t}_{0}$ for filtration operations from the data file. A minute or two of variation during this highly dynamic time period could bias the measured $\mathrm{R}_{\text {tot }}$.

Thus, it appears that for the cleaning performed for the Series 1 tests, the impact of any residual membrane resistance cannot be separated from the inherent variability at the start of filtration operations on an acid-cleaned filter. One standard technique for reducing this variability is to fill the permeate lines and backpulse the filter once before the start of filtration. This technique was not used for the Series 1 tests because of the potential for biasing the long-time fouling behavior by applying some amount of filter conditioning prior to the start of the test.

\subsection{Series 2 Cleaning Test Results}

The test results for CWF measurements associated with Series 2 cleaning activities (see Section 3.3.2) are shown in Figure 7.4. In many respects, the CWF test results are comparable to those observed for Series 1. Initial pre-test CWF are generally high, ranging from $\sim 1$ to $2.8 \mathrm{GPM} / \mathrm{ft}_{2}$, but show rapid decline to fluxes that range from 0.2 to $0.5 \mathrm{GPM} / \mathrm{ft}^{2}$. The initial pre-test $\mathrm{CWF}$ for $\mathrm{S} 2 \mathrm{~T} 1$ is exceptionally high, starting at $2.8 \mathrm{GPM} / \mathrm{ft}^{2}$, which is well beyond the expected maximum calculated in Section 7.1. This result is not entirely unexpected, as a new filter element was installed on the CUF filtration system before Series 2 testing. Post-test CWF flux shows a strong component dependence. Initial post-test flux values and values after flux decline correspond to the observed component dependence for Series 2 testing. That is, CWF measurements following tests with $\mathrm{FeOOH}$ and $\mathrm{CrOOH}$ solids (tests $\mathrm{S} 2 \mathrm{~T} 2$, S2T6, and S2T8) show low flux relative to CWF measurements following tests with boehmite solids (S2T1 and S2T4).

Series 2 testing provided an opportunity to look at component fouling and evaluate cleaning strategies for each component. The overall observation made during testing (described in Section 0) was that chrome oxy-hydroxide $(\mathrm{CrOOH})$ and iron oxy-hydroxide $(\mathrm{FeOOH})$ are the key depth-fouling constituents, and both require acid cleaning to restore a CWF that compares to a new filter baseline flux. In the case of S2T8 and S2T6, the known effective cleaning agent, oxalic acid, was used to prepare the filter for the next test. For S2T2, a progressive cleaning regimen with nitric acid and oxalic acid was used to attempt to quantify and compare the effectiveness of the two agents. The results of this test are presented in Section 7.4. The boehmite simulants at two different PSD ranges did not appear to cause appreciable decline in filter flux relative to flux loss from trace solids held-up in the system. Simple backpulsing of these simulants at the concentrations tested was sufficient to restore CWF to above $1 \mathrm{GPM} / \mathrm{ft}^{2}$. Though arguably not required, nitric acid cleaning was performed after the fine boehmite test (S2T4) for removing any irreversible fouling.

All of the simulant solids were at small volumetric concentrations relative to their concentrations in the actual PEP simulant. While the time frames and number of particles involved were sufficient to provide a measureable amount of fouling, the extent of irreversible fouling was less than that observed for 
the blended simulant at a higher concentration. In evaluating component fouling and cleaning, it is necessary to consider the multiplicity of component interactions and not necessarily view the fouling behavior as merely a sum of the parts or to define it in terms of the most significant contributor. For instance, the boehmite might function as a filtration aid by setting up a protective cake and mitigating depth fouling by $\mathrm{FeOOH}$ and $\mathrm{CrOOH}$. Other components may adhere to and help draw out depth-fouled particles during cleaning. Such interactions are difficult to verify and quantify. These considerations provide a necessary caveat for observations and conclusions from this cleaning data.

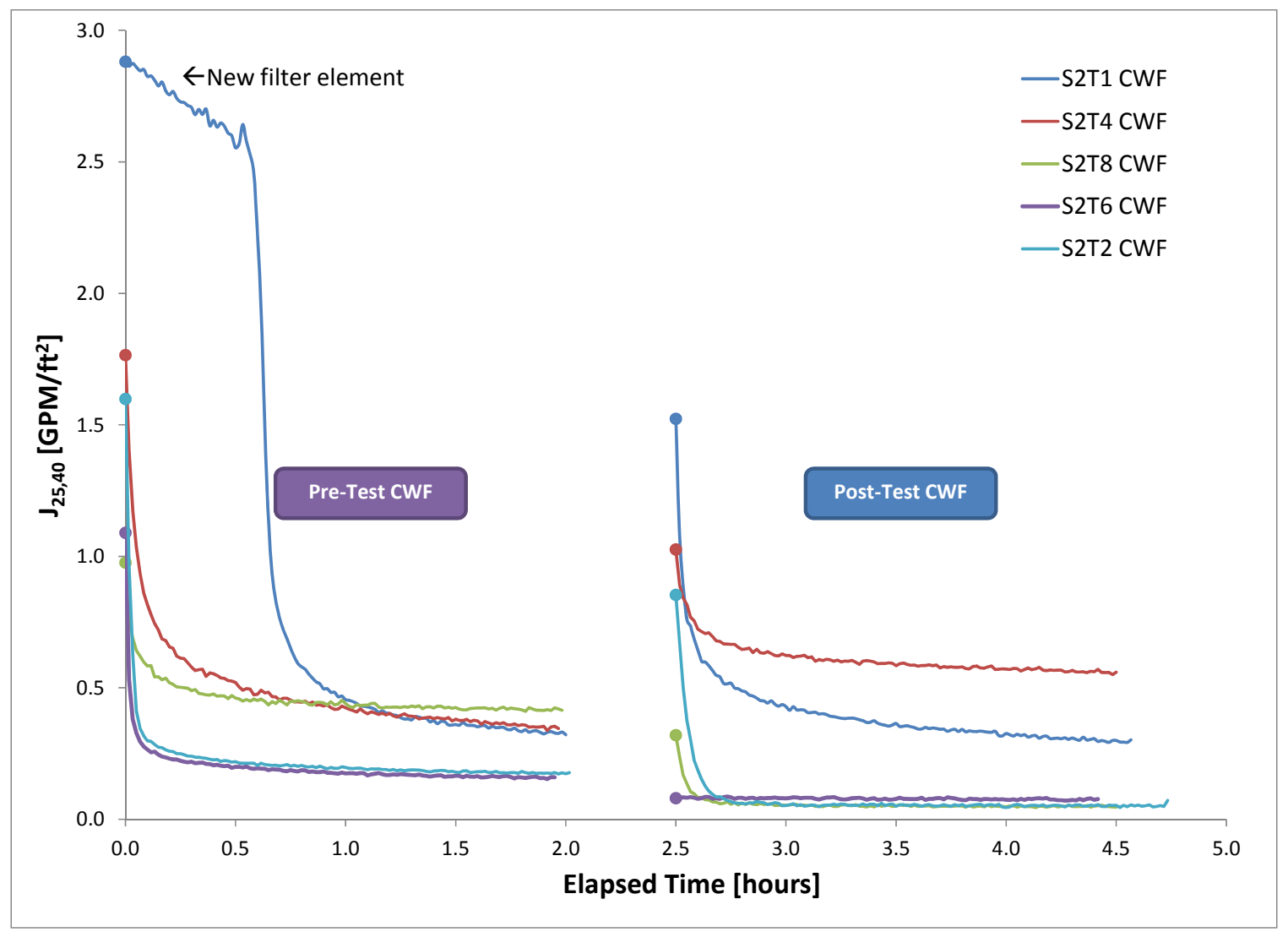

Figure 7.4. Clean Water Flux Before and After Each Series 2 Test (flux corrected to a TMP of 40 psid)

\section{4 $\mathrm{SrCO}_{3}$ Cleaning Test Results}

The flux measured during this series of tests (scaled/corrected to TMP target of $40 \mathrm{psid}$ ) is shown in Figure 7.5, Figure 7.6, and Figure 7.7. The condensed elapsed time axis is a continuous time axis that eliminates dead time between the various steps, particularly "overnight" periods where no testing was performed. Reviewing the results of the testing in Figure 7.5, two key observations emerge.

The first observation is that the second and third $\mathrm{SrCO}_{3}$ tests have nearly the same flux. This is made more obvious in Figure 7.6. The post-nitric and post-oxalic $\mathrm{SrCO}_{3}$ flux curves are indistinguishable, indicating that a) the oxalic acid did not improve the filter flux beyond the level seen for nitric acid and b) $\mathrm{SrCO}_{3}$ flux measurements provide a cleaning metric that is more stable (with respect to transient flux decay) than the CWF measurements, such as those reported in Sections 7.2 and 7.3. 
On the first point, it is curious that nitric acid was equally successful in cleaning the element as measured by the $\mathrm{SrCO}_{3}$ flux. This flux was fivefold higher than the flux for the conditioned filter (before acid cleaning), indicating that significant membrane resistance was removed by nitric acid cleaning. This has not been previously observed for blended filtration simulants, where amorphous FeOOH has typically been implicated as the primary depth fouling component that most needed and responded best to oxalic acid cleaning. It is possible that this observed effect is due to the low concentration of $\mathrm{FeOOH}$ or to differences in the suspending medium during filtration testing. It is also possible that $\mathrm{FeOOH}$ does not contribute to nitric-acid-resistant irreversible fouling in the way it is currently understood. There may be other components and component interactions in the simulant that lead to this type of fouling that are not known at this time. Destructive analysis of a fouled filter may provide additional insight into the composition of depth-fouled solids.

On the second point, the current 2-hour CWF studies highlight the sensitivity of CWF measurements to trace solids that are likely held-up in dead volumes within the test system, which are the suspected cause for the rapid, reversible decay of the flux observed in CWF testing. Use of $\mathrm{SrCO}_{3}$ slurry for filter cleanliness benchmarks effectively masks these trace solids or organic impurities to yield repeatable measurements of filter conditioning. This has been previously observed in Geeting et al. (2003) and Nash et al. (2000).

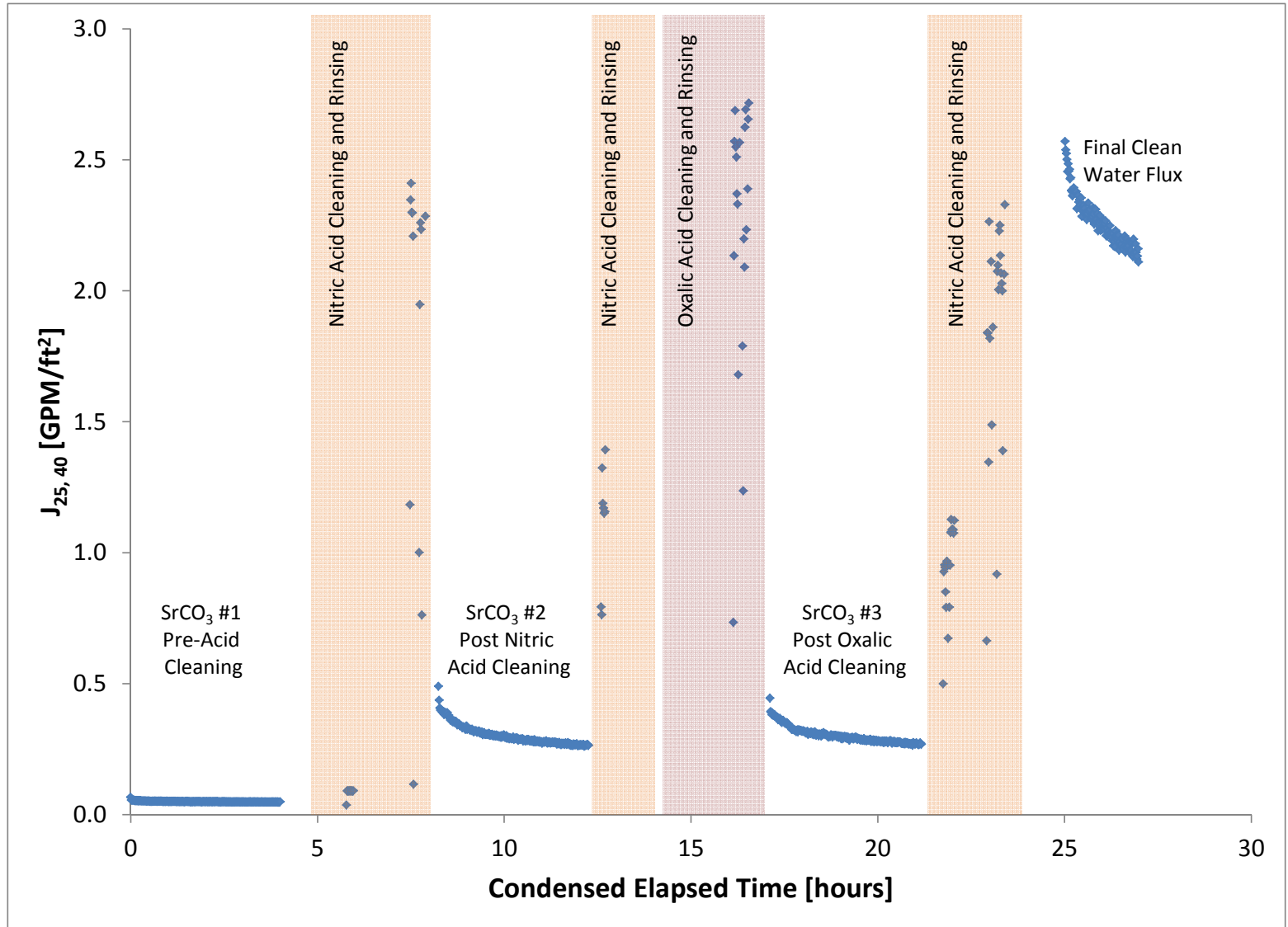

Figure 7.5. Flux During $\mathrm{SrCO}_{3}$ Slurry Testing Following Series 2, Test 2 (FeOOH, 0.1 vol\%) 


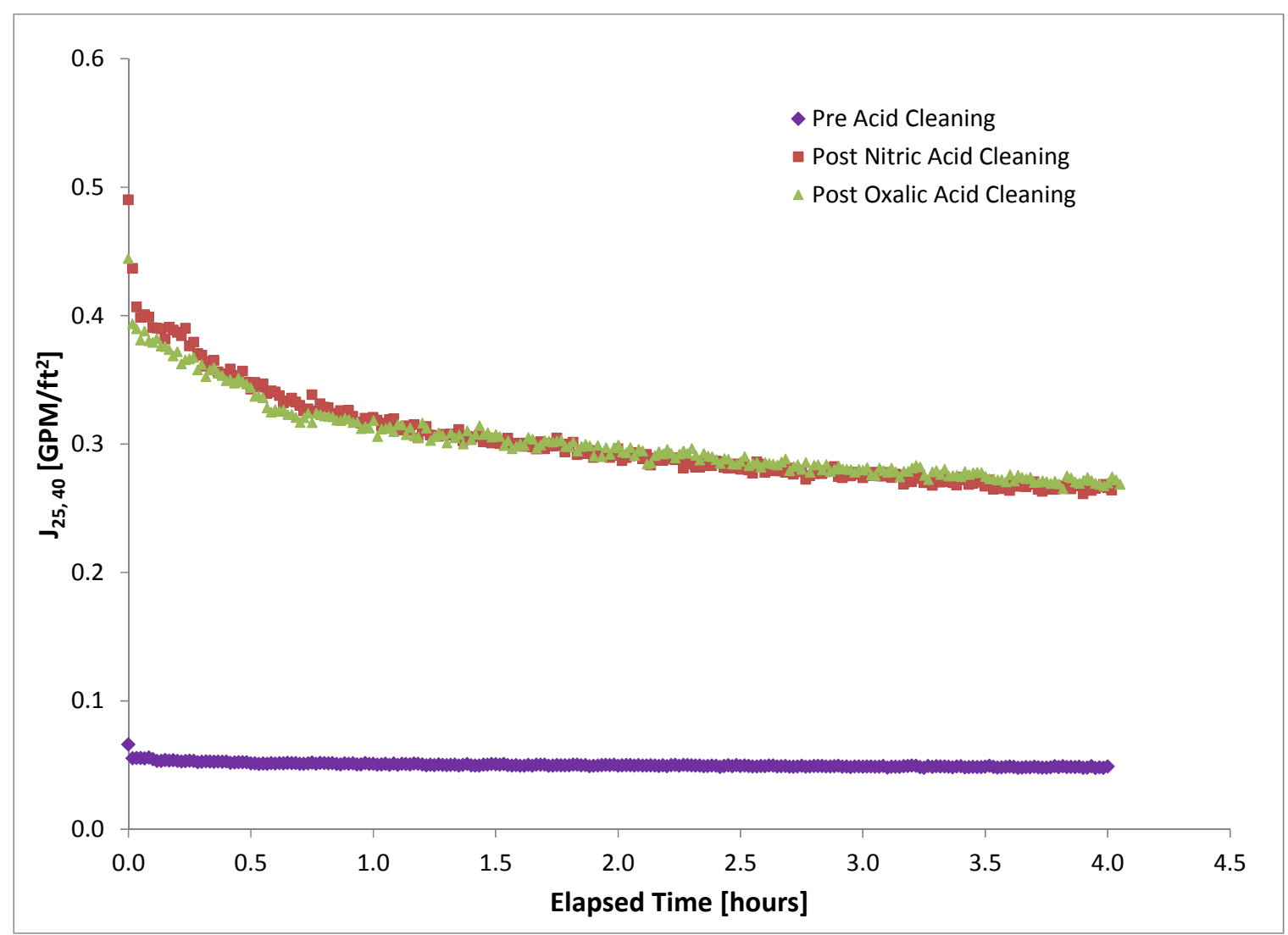

Figure 7.6. Comparison of Flux During the Three $\mathrm{SrCO}_{3}$ Tests in the Cleaning Sequence. Tests \#2 (post-nitric acid cleaning) and \#3 (post-oxalic acid cleaning) are virtually indistinguishable.

The second observation is that the final CWF is exceptionally high and stable. This test represents the maximum CWF obtained in any of the historical simulant and waste tests. In fact, as described earlier, this value was the basis for selecting $2.5 \mathrm{GPM} / \mathrm{ft}^{2}$ (TMP corrected to $40 \mathrm{psid}$ ) as the maximum CWF for this filter type. Two reasons why this TMP-corrected flux was obtained during the current testing include 1) use of a relatively new filter element and 2) testing of $\mathrm{SrCO}_{3}$ slurry before $\mathrm{CWF}$ testing helps sequester trace solids that remain after acid cleaning with the $\mathrm{SrCO}_{3}$ that is drained from the system. In effect, testing with the $\mathrm{SrCO}_{3}$ slurry provides a means of removing trace solids from the filter system. The effectiveness of $\mathrm{SrCO}_{3}$ as a "cleaning" agent was observed in previous tests (Geeting et al. 2003). 


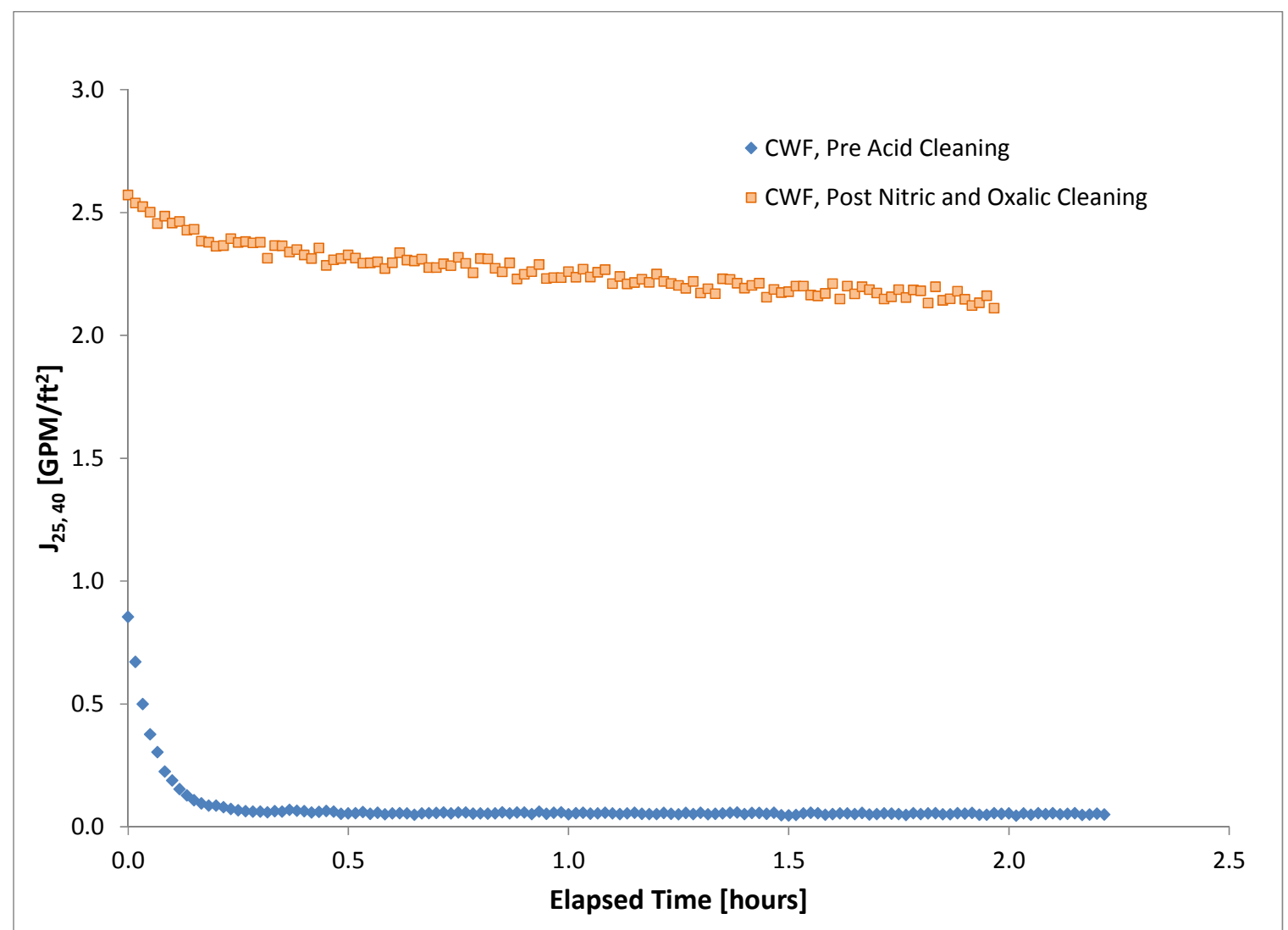

Figure 7.7. Clean Water Flux Measurements Made During $\mathrm{SrCO}_{3}$ Cleaning Tests. The pre-acid cleaning CWF measurement follows Series 2, Test $2(\mathrm{FeOOH}, 0.1 \mathrm{vol} \%)$. The final CWF test follows nitric acid cleaning, oxalic acid cleaning, and several periods of filtration of a $\mathrm{SrCO}_{3}$ slurry.

\subsection{Series 3 Test Conclusions}

In this section, CWF test results associated with Series 1, Series 2, and $\mathrm{SrCO}_{3}$ testing have been presented. Several questions that derive from these CWF results are:

- What is the best measure for CWF flux given the rapid decline? If reversible fouling by trace particulates is the suspected cause of flux decline (and this appears to be the case), then the CWF measured immediately following backpulsing would seem to be the most appropriate CWF measurement to assess filter cleanliness.

- Is there any impact of from trace contaminants on the initial filter flux measured for simulant? If the trace contaminants depth fouls the filter, then it is likely that it would contribute to the overall filter resistance and impact the initial flux measured for PEP simulant. On the other hand, if the trace contaminant fouling is reversible, then it is likely that any resistance offered by the particles is indistinguishable from that of the simulant, especially since the trace contaminants are most likely remnants of the simulant itself. If the latter is the case, then it again points to the initial (post-backpulse) value of CWF as being indicative of filter cleanliness.

Overall, these questions point to the difficulty in identifying a CWF benchmark in systems contaminated with trace particles. Based on the arguments above, the best approach appears to take the 
initial value (immediately following the start of filtration operations or following a backpulse) as the CWF used to assess filter cleanliness. The rationale for this choice is that the backpulsed CWF provides the flux that can be achieved after all reversible foulants have been removed, as these are not expected to provide a distinguishable resistance when combined with slurry solids.

Given this conclusion, it is possible to assess the overall impact on the expected initial flux of a PEP simulant slurry. Based on Series 1 testing, a best achievable starting flux of $0.1 \mathrm{GPM} / \mathrm{ft}^{2}$ is assumed. It is believed that this assumption is reasonable, since filters that yielded acceptably high CWF (with respect to the history $1 \mathrm{GPM} / \mathrm{ft}^{2}$ benchmark) generally produce fluxes near $0.1 \mathrm{GPM} / \mathrm{ft}^{2}$ for the PEP simulant. Filter flux of the expected PEP simulant, $J_{P E P}$, is modeled using:

$$
J_{P E P}=\frac{\Delta P}{\mu\left(R_{m}^{o}+R_{r e s}+R_{\text {sim }}\right)}
$$

Here $R_{\text {res }}$ is the residual fouling of the filter not removable by acid cleaning and $R_{\text {sim }}$ is the expected initial fouling of the PEP simulant. These terms are analyzed from the measured value for CWF, $\mathrm{J}_{\mathrm{CWF}}$, and from the maximum initial PEP flux, $J_{P E P, \max }$, of $0.1 \mathrm{GPM} / \mathrm{ft}^{2}$ using:

and

$$
J_{P E P, \max }=\frac{\Delta P}{\mu\left(R_{m}^{o}+R_{\text {sim }}\right)}
$$

$$
J_{C W F}=\frac{\Delta P}{\mu\left(R_{m}^{o}+R_{r e s}\right)}
$$

Finally, $R_{m}{ }^{o}$ taken from Table 7.2 corresponds to a maximum clean water flux of $2.1 \mathrm{GPM} / \mathrm{ft}^{2}$. Application of this analysis allows calculation of the expected PEP flux values and deviation from the maximum flux of $0.1 \mathrm{GPM} / \mathrm{ft}^{2}$ for various measured values of $\mathrm{CWF}$.

Figure 7.8 shows the results of the residual fouling consequence analysis. First consider the case of an initial CWF of $1 \mathrm{GPM} / \mathrm{ft}^{2}$, which is the minimum flux considered historically acceptable. Figure 7.8 says that residual fouling will reduce the PEP simulant flux by $14 \%$, resulting in a PEP flux of $0.086 \mathrm{GPM} / \mathrm{ft}^{2}$. Given that CWF testing for Series 1 showed that acid cleaning could always achieve initial fluxes greater than $1 \mathrm{GPM} / \mathrm{ft}^{2}$, the overall consequence of residual fouling is less than a $14 \%$ decrease in flux from ideal based on this analysis. It should be noted that if the assumed best flux for PEP were taken to be lower than $0.1 \mathrm{GPM} / \mathrm{ft}^{2}$, then the deviation in initial flux caused by a $1 \mathrm{GPM} / \mathrm{ft}^{2}$ would be lower. This is important, as initial filter flux for actual Hanford wastes generally fall below $0.1 \mathrm{GPM} / \mathrm{ft}^{2}$ (Fiskum et al. 2009; Edwards et al. 2009, Shimskey et al. 2009a; Shimskey et al. 2009b; Lumetta et al. 2009). As such, the estimated $14 \%$ deviation from ideal waste or simulant associated with a $1 \mathrm{GPM} / \mathrm{ft}^{2}$ benchmark may be conservative such that deviation for actual waste slurries may be less.

In the above case, the assumption of a $1 \mathrm{GPM} / \mathrm{ft}^{2} \mathrm{CWF}$ and $0.086 \mathrm{GPM} / \mathrm{ft}^{2} \mathrm{PEP}$ flux is consistent with the case of an acid-cleaned filter. In contrast, when filter elements that have been exposed to PEP simulant slurry are rinsed but not acid cleaned, initial fluxes are generally $0.5 \mathrm{GPM} / \mathrm{ft}^{2}$ or greater. For a CWF of $0.5 \mathrm{GPM} / \mathrm{ft}^{2}$, deviations of up to $32 \%$ can be expected (with initial PEP fluxes reduced to $0.068 \mathrm{GPM} / \mathrm{ft}^{2}$ ). Based on this information, it can be concluded that acid cleaning would increase the initial Series 1 flux by $\sim 26 \%$. 


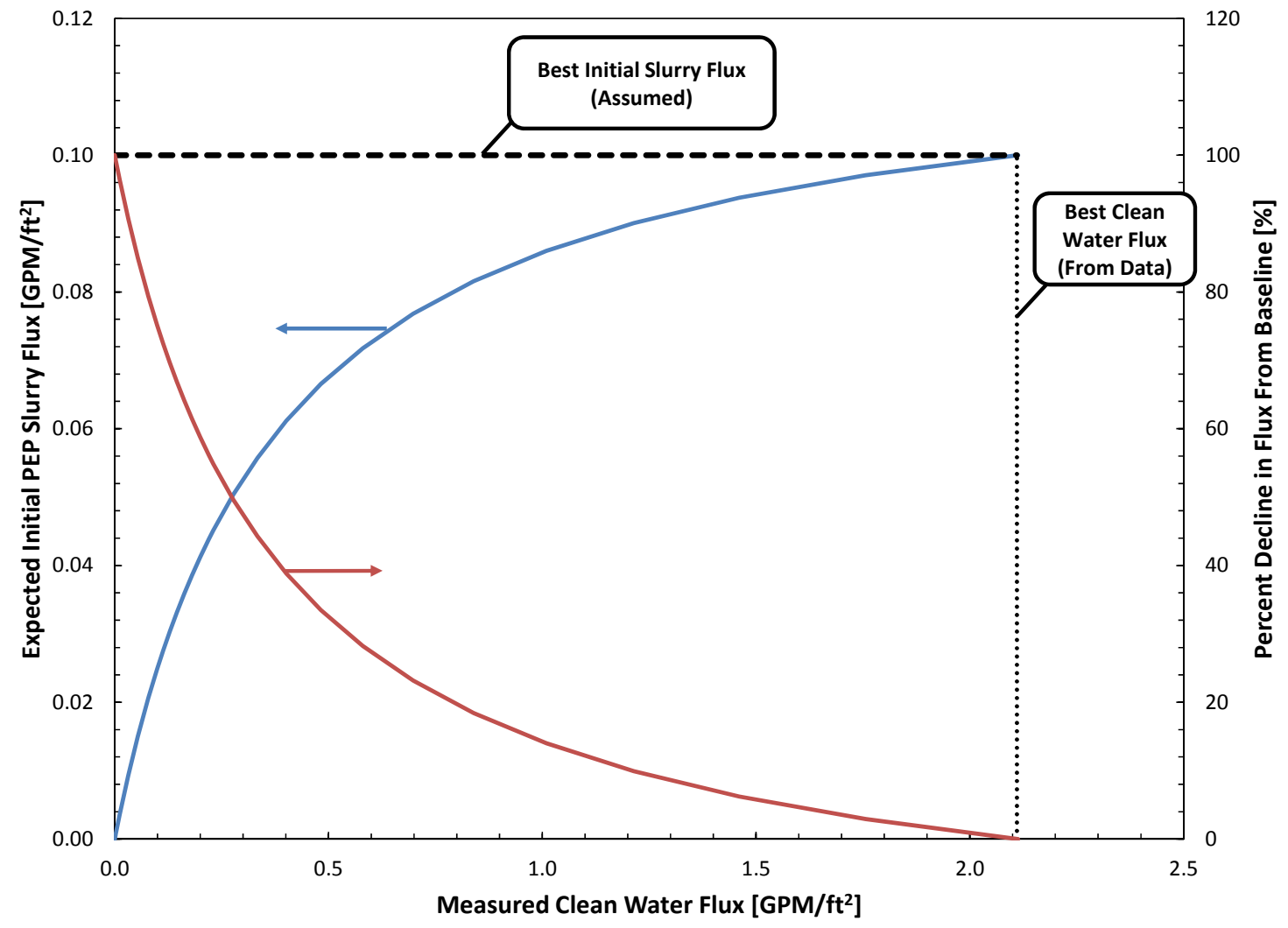

Figure 7.8. Expected Impact of Fouling Not Removed by Cleaning (as expressed in terms of measured CWF of cleaned filter) on the Initial Filter Flux for a PEP Simulant Slurry That Has a Best Initial Flux of $0.10 \mathrm{GPM} / \mathrm{ft}^{2}$

Given the assumptions and approach outlined above, acid cleaning for Series 1 was postulated to provide a $\sim 26 \%$ increase in initial flux or greater. While this increase may seem significant, analysis of acid cleaning effectiveness must also consider the following factors:

- acid cleaning requires suspension of filter operations, which translates to lost throughput

- the $26 \%$ increase in flux with acid cleaning only corresponds to initial values, and for the acid cleaned and rinsed only flux curves should converge as the filter fouls as a result of long-time fouling dynamics.

Examination of Series 1 test results presented in Section 5 indicates that the $26 \%$ increase calculated above will be eliminated after approximately 1 hour of filtration operations at 5 -wt $\%$. Thus, the effectiveness of acid cleaning after each Series 1 test relative to rinsing appears suspect given that the acid cleaning step alone took 30 minutes.

It is important to note that while the effectiveness of acid cleaning in increasing filter throughput for Series 1 testing may be suspect, this concern only applies to the 100-hour test format applied for Series 1 testing. As stated in the conclusions to Section 5, there is still significant uncertainty with respect to the impact of backpulsing on irreversible flux loss. Specifically, the current results do not indicate if continued backpulsing and/or operation of the filters beyond 100 hours will lead to a complete blocking of the filter (i.e., zero flux) or if some steady-state level of irreversible fouling with respect to backpulsing 
will be achieved. Without this information, the need for and effectiveness of acid cleaning cannot be fully assessed.

CWF tests for Series 1 and Series 2 do not speak to the effectiveness of oxalic and nitric acids. As discussed in Section 1.3, use of oxalic acid in simulant testing has been necessitated due to the inability of nitric acid to restore filter flux to pre-test CWF levels for filters that have been contacted with PEP simulant slurry. Series 2 testing appears to identify FeOOH solids as the major fouling species present in the baseline PEP formulation. As such, $\mathrm{SrCO}_{3}$ cleaning tests that followed Series 2 component tests with FeOOH solids provided an excellent opportunity to evaluate the effectiveness of filter cleaning with nitric and oxalic acid. For the $\mathrm{FeOOH}$ simulant component, cleaning with nitric acid alone appears to restore flux effectively. Additional cleaning with oxalic acid did not produce a measurable increase in flux beyond that provided by nitric acid. As such, the inability of nitric acid to restore flux in previous filtration studies does not appear associated with difficulty in removing FeOOH solids. In addition, Series 2 testing appears to rule out boehmite solids, which generally show filter fluxes that are one order of magnitude above that of the PEP simulant. Given this information, it appears that difficulties in nitric acid cleaning are not caused by the major simulant components. Additional study is required to identify what components foul the filter element. One way to address this need is additional component testing with minor simulant components. However, it may be more useful to destructively examine the filter element to see how individual simulant components interact with the filter. Specifically, direct imaging of the filter surface with optical microscopy or SEM may provide information on the composition of particulates that foul the filter element.

Overall, the following conclusions can be made with respect to Series 3 cleaning:

- The best clean water flux achievable for the Mott Grade 0.1 tubular filter elements used in CUF testing falls between 2-3 GPM/ $\mathrm{ft}^{2}$. It should be noted that this level of flux was achieved when testing with new filters or filters cleaned extensively with a combination of nitric acid, oxalic acid, and $\mathrm{SrCO}_{3}$ slurry contacts.

- After extended contact with PEP simulant slurry, filter cleaning with $0.5 \mathrm{M}$ oxalic acid produced initial values for CWF that ranged from 1.2 to $1.8 \mathrm{GPM} / \mathrm{ft}^{2}$. However, trace solids not removed by acid cleaning cause a rapid decline in the observed CWF.

- Cleaning tests associated Series 2 show CWF decays similar to those observed for Series 1 CWF tests. Series $2 \mathrm{CWF}$ show that trace solids for $\mathrm{FeOOH}$ and $\mathrm{CrOOH}$ simulant components are capable of significantly reducing flux, even at low concentrations. It can be postulated that these trace solids also cause flux decline in CWF measurements associated with Series 1 testing.

- The initial CWF flux (i.e., that immediately following start-up of filter operations or immediately following a backpulse) appears to be an appropriate measure for filter cleanliness and avoids the need to accommodate flux decline from residual solids.

- If the initial (post-backpulse) CWF measurement exceeds $1 \mathrm{GPM} / \mathrm{ft}^{2}$, which has been the historical benchmark for "acceptable" CWF, it is estimated that the deviation initial flux for a PEP simulant slurry is less than $14 \%$. This estimation is based on an ideal slurry flux of $0.1 \mathrm{GPM} / \mathrm{ft}^{2}$ at low solids concentrations. For waste slurries (on relatively clean filters) that show filter performance behaviors well below this mark, the actual deviation in flux associated with a $1 \mathrm{GPM} / \mathrm{ft}^{2} \mathrm{CWF}$ benchmark is expected to be lower. 
- The effectiveness of acid cleaning with respect to rinsing without acid cleaning is suspect. Estimates indicate that acid cleaning increased flux by $26 \%$ relative to rinsing alone; however, this flux increase is temporary and may correspond to only a few hours of increased filter throughput when compared to rinsing. However, this argument is based on the Series 1 test format and may fail at longer durations.

- CWF testing was not able to associate poor historical performance of nitric acid in restoring filter flux to any major simulant component. For cleaning of $\mathrm{FeOOH}$ solids, which were identified as a major contributor to the PEP simulant fouling behavior, nitric acid cleaning was effective at restoring flux performance of $\mathrm{SrCO}_{3}$. Cleaning with oxalic acid did not provide any additional benefit in terms of $\mathrm{SrCO}_{3}$ flux performance.

- Information on the ultimate fate of filter flux is still needed to evaluate the need for acid cleaning. It is still not known if repeated backpulsing of the filter or long-time filtration will irreversibly foul the filter until the permeate flux reaches zero or if irreversible fouling will reach a steady state. For the former case, acid cleaning will eventually be needed to clear the irreversible fouling. In the latter case, it may be more effective to simply maintain flux through backpulsing operations. 


\subsection{Filtration Model Evaluation and Update}

A series of analyses was performed on the Series 1 filtration data to determine how to model filtration behavior over long periods of operation. The investigation was focused on the origin of the long-term flux decay observed in the tests, as shown in Section 5.2. Long-term flux degradation (i.e., no discernable approach to a steady flux) is not commonly observed in cross-flow filtration systems, and it is important to understand this behavior, as it governs how the filters should be operated during waste treatment in order to maximize throughput.

In the first section, the Series 1 data is first using an existing, PNNL-developed model. A couple of modifications to the model are also discussed. In the next section, a different modeling approach, derived from well-known mechanisms, is described. The third section discusses the implications of the model for filter operations, and the final section explains the limitations of the model and suggests future testing to mitigate their effects.

\subsection{Comparison of Data to Existing Filter Flux Model}

First, a model developed by Daniel et al. (2010b) using data from shorter tests ${ }^{5}$ using the same simulant material was tested against the Series 1 data. The model treats filter fouling by assuming that two separate processes yield a decrease in the filter performance: pore fouling of the filter element and cake formation. The effects of pore blocking and cake formation are expressed in terms of increasing membrane and cake resistance terms, respectively. The membrane resistance, $R_{m}$, changes with time according to the following expression:

$$
\frac{d R_{m}}{d t}=k_{m} J R_{m}^{2}
$$

Here, $t$ is elapsed filtration time and $J$ is filter flux (recall that $J$ is filtered and normalized as described in Section 4.3). All the physical properties and operational parameters have been incorporated into the effective capture-rate constant, $k_{m}$. The term on the right-hand side of Equation 8.1 captures the convective flux and capture of particles into the porous filter membrane. Likewise, the change in the cake resistance, $R_{c}$, with time is modeled by

$$
\frac{d R_{c}}{d t}=k_{c 1} J-k_{c 2}
$$

As was the case for $k_{m}$, the constants $k_{c 1}$ and $k_{c 2}$ incorporate both system operational parameters and physical properties (such as the proportionality constant between cake thickness and resistance). The first right-hand-side term approximates the convective flux and capture of particles on the filter surface, while

5 The shorter tests had three segments: 12 hours of continuous filtration, followed by 12 hours of backpulsing every 30 minutes, and then 12 more hours of continuous filtration. 
the second right-hand term captures erosion of the cake by fluid shear. Equations for the rate of change in membrane and cake resistance are coupled through the filter flux, assuming a Darcy's law relationship:

$$
J=\frac{\Delta P}{\mu\left(R_{m}+R_{c}\right)}
$$

where $\mu$ is the liquid-phase viscosity and $\Delta P$ is the TMP. ${ }^{6}$ Predicting flux dynamics associated with combined pore blocking and cake formation requires simultaneously solving Equations 8.1 and 8.2. To facilitate a solution, it is assumed that filter flux is characterized by an initial flux $J_{o}$. Thus, we have the initial conditions defined as:

$$
\begin{aligned}
\left.J\right|_{t=0} & =J_{o}, \\
\left.R_{m}\right|_{t=0} & =R_{m 0}=\frac{\Delta P}{\mu J_{o}}, \text { and } \\
\left.R_{c}\right|_{t=0} & =0 .
\end{aligned}
$$

The initial conditions given in Equation 8.4 assume that an initial flux can be specified and there is no cake resistance when the filter is initially in operation.

Before the model of Equations 8.1 through 8.4 was fit to the Series 1 flux data, it was rendered dimensionless. Numerical schemes typically perform better (are more stable and converge quicker) when the equations and parameters are all of the same order, which is often the case with dimensionless variables. Thus, the following dimensionless variables were defined:

$$
J^{*}=\frac{J}{J_{o}} ; R_{m}{ }^{*}=\frac{R_{m}}{R_{m 0}} ; R_{c}^{*}=\frac{R_{c}}{R_{m 0}} ; \text { and } t^{*}=\frac{t \mu J_{o}{ }^{2} k_{c 1}}{\Delta P} \text {. }
$$

Note that one of the fitting parameters $\left(k_{c 1}\right)$ is now incorporated into the time scale. The choice of dimensionless variables gives the following dimensionless equation system upon substitution:

$$
\begin{gathered}
J^{*}=\frac{1}{R_{m}^{*}+R_{c}^{*}}, \\
\frac{d R_{m}^{*}}{d t^{*}}=\beta J^{*} R_{m}^{* 2}, \text { and } \\
\frac{d R_{c}^{*}}{d t^{*}}=J^{*}-K_{c},
\end{gathered}
$$

${ }^{6}$ TMP will be used as an abbreviation for the term transmembrane pressure, whereas $\Delta P$ will be used to refer to TMP in mathematical expressions. 
where $K_{c}=k_{c 2} / k_{c 1} J_{o}$ and $\beta=\left(k_{m} / k_{c 1}\right)\left(\Delta P / \mu J_{o}\right)^{2}$, subject to the boundary conditions:

$$
\begin{gathered}
\left.J^{*}\right|_{t^{*}=0}=1, \\
\left.R_{m}^{*}\right|_{t^{*}=0}=1 \text {, and } \\
\left.R_{c}^{*}\right|_{t^{*}=0}=0 .
\end{gathered}
$$

The recast equation system now has a cake "equilibrium" constant $\left(K_{c}\right)$ that measures the ratio of the two cake resistance mechanisms and a parameter $\beta$ that compares membrane and cake resistance constants.

The dimensionless model was fit to the Series 1 data using a non-linear least squares minimization routine. The coefficients obtained for each minimization are shown in Table 8.1. The model describes the flux behavior adequately, but only after approximately 5 hours has elapsed (for example, see Figure 8.1). The short-time behavior was not modeled very well by Equations $8.5-8.8$. Furthermore, the model generates some physically unexpected behavior of the resistances (see Figure 8.4). The model predicts that the membrane resistance $R_{m}$ increases exponentially with filtration time, while the cake resistance has a maximum value at $\sim 50$ hours of filtration time and then decreases rapidly. There is nothing to suggest that the cake would suddenly erode after 50 hours of operating the cross-flow filter at constant TMP and AV, nor should the membrane resistance increase at a faster rate when there is a cake covering the porous surface of the filter. It is expected that the presence of a cake would "protect" the membrane from particles (or at least a fraction of particles) that would foul the pores in the absence of a cake.

The model insinuates that cake formation and maturation (as measured via $R_{c}$ ) is a function of TMP and AV. For example, the cake equilibrium constant $K_{c}$, appears to scale linearly (see Figure 8.2) with the ratio of the transmembrane pressure to the inertial pressure $\Lambda=\Delta P / \rho U^{2}$, which would be expected if cake formation was an important mechanism. ${ }^{7}$ TMP drives particles toward the surface of the filter where they can assemble into a cake, whereas AV erodes the cake by re-suspending particles from the cake back into the bulk. The relationship was found to be linear when $1 / K_{c}$ is normalized by the flux at 10 hours. The same relationship would appear to hold when $J_{o}$ is used to compute $1 / K_{c}$, except for test S1T3. This is suspected to be low because the initial flux was unusually low for this test (especially when compared with S1T1 and S1T2). This relationship could be used to predict $K_{c}$ at other TMP and AV within the range of $\Lambda$ tested. Note that $1 / K_{c}$ is $>1$ for all Series 1 tests, indicating that the cake should continue to increase $R_{c}$ over time. This was not observed in the model fit (e.g., Figure 8.4).

${ }^{7}$ Note that $\mathrm{U}$ is the area-average slurry velocity, given by $\mathrm{U}=\mathrm{Q} /\left(\pi \mathrm{D}^{2} / 4\right)$, where $\mathrm{Q}$ is the volumetric flow rate of slurry and $\mathrm{D}$ is the diameter of the filter element. 


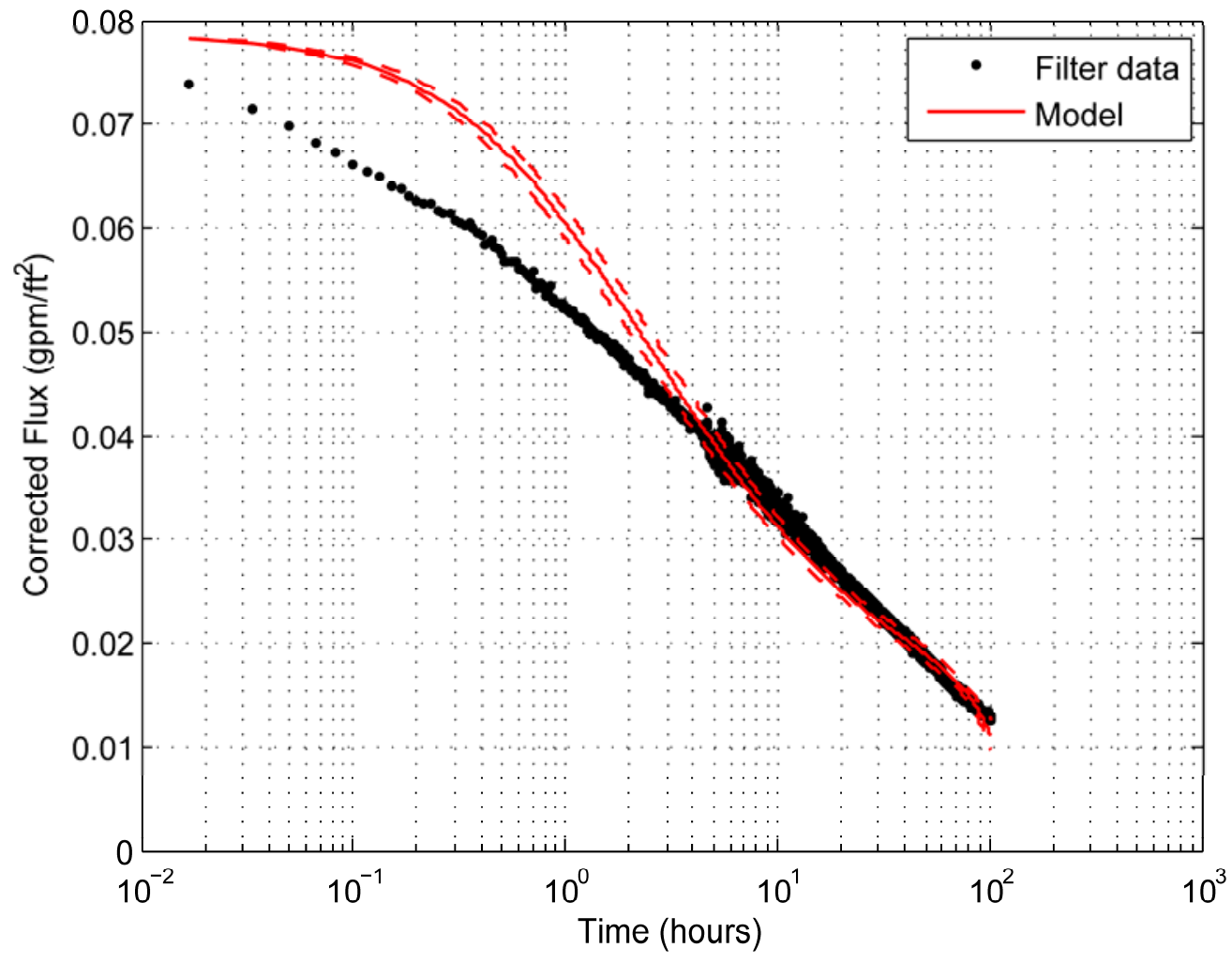

Figure 8.1. Original Model Fit to S1T1 Data before Backpulsing ( 100 hours). The dotted lines are the bands of $95 \%$ confidence for the model fit.

Table 8.1. Results of the Non-Linear Regression Applied to the Series 1 Data

\begin{tabular}{cccccc}
\hline Test & S1T1 & S1T2 & S1T3 & S1T4 & S1T5 \\
\hline$\beta$ & 0.0694 & 0.0600 & 0.0869 & 0.0559 & 0.0688 \\
$\mathrm{~K}_{\mathrm{c}}$ & 0.2546 & 0.3124 & 0.2566 & 0.1745 & 0.4299 \\
$\mathrm{k}_{\mathrm{m}}$ & $8.79 \times 10^{-14}$ & $7.64 \times 10^{-14}$ & $8.24 \times 10^{-14}$ & $8.32 \times 10^{-14}$ & $1.07 \times 10^{-13}$ \\
$\mathrm{k}_{\mathrm{c} 1}\left(\mathrm{~m}^{-2}\right)$ & $4.30 \times 10^{12}$ & $3.98 \times 10^{12}$ & $4.96 \times 10^{12}$ & $8.20 \times 10^{12}$ & $2.50 \times 10^{12}$ \\
$\mathrm{k}_{\mathrm{c} 2}\left(\mathrm{~m}^{-1} \mathrm{~s}^{-1}\right)$ & $5.85 \times 10^{7}$ & $6.93 \times 10^{7}$ & $5.48 \times 10^{7}$ & $9.00 \times 10^{7}$ & $4.18 \times 10^{7}$ \\
$\mathrm{SSE}^{(\mathrm{a})}$ & 2.262 & 2.503 & 1.509 & 2.504 & 1.863 \\
$\mathrm{MSE}^{(\mathrm{b})}$ & $3.74 \times 10^{-4}$ & $4.14 \times 10^{-4}$ & $2.52 \times 10^{-4}$ & $4.17 \times 10^{-4}$ & $3.11 \times 10^{-4}$ \\
$\Lambda$ & 10.71 & 8.34 & 14.26 & 16.06 & 5.35 \\
\hline
\end{tabular}

(a) Sum of squared errors of prediction.

(b) Mean squared error. 


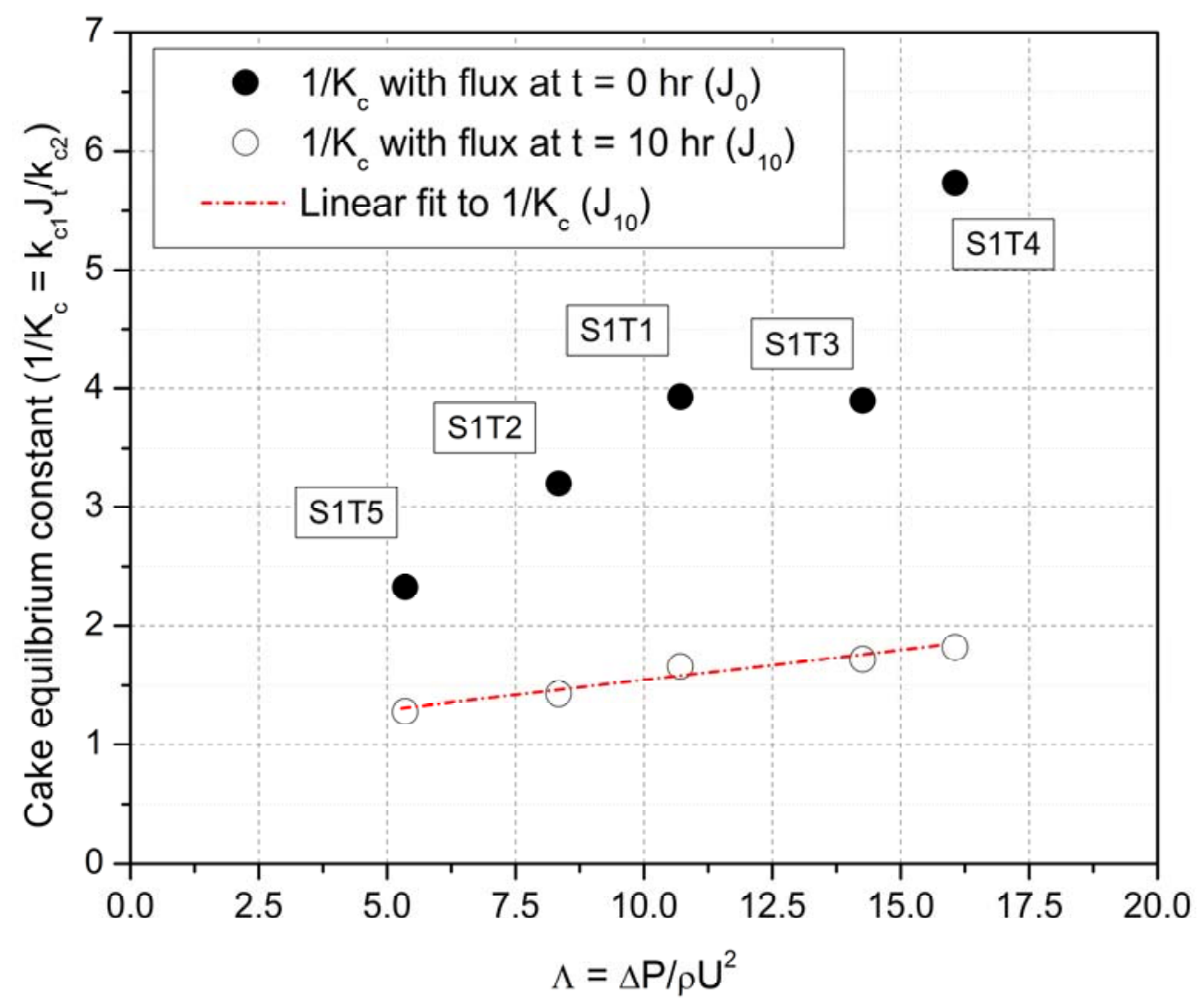

Figure 8.2. Cake Equilibrium Constant vs. Dimensionless Pressure Ratio $(\Lambda)$ for All Series 1 Tests. The solid circles use the initial flux and the open circles use the flux at $t=10$ hours in the equilibrium constant. Note that it appears a linear fit would also be valid for the $\mathrm{K}_{\mathrm{c}}\left(\mathrm{J}_{0}\right)$ data but for the one outlier (from test S1T3).

It is important to point out that the existing PNNL model fit flux data from the first 12 hours of filtration quite well (see Figure 8.3). However, when the parameters that fit 12 hours of data are extrapolated to longer times, the flux is significantly underpredicted. For instance, at the end of test $\mathrm{S} 1 \mathrm{~T} 1$, the measured flux was $8.65 \times 10^{-6} \mathrm{~m} / \mathrm{s}$. The 12 -hour fit projects the flux to be $7.0 \times 10^{-11} \mathrm{~m} / \mathrm{s}$, i.e., essentially zero; even at only 24 hours, the projected flux is only $4 \%$ of the measured flux for S1T1. Similar underprediction was observed for all five tests. 


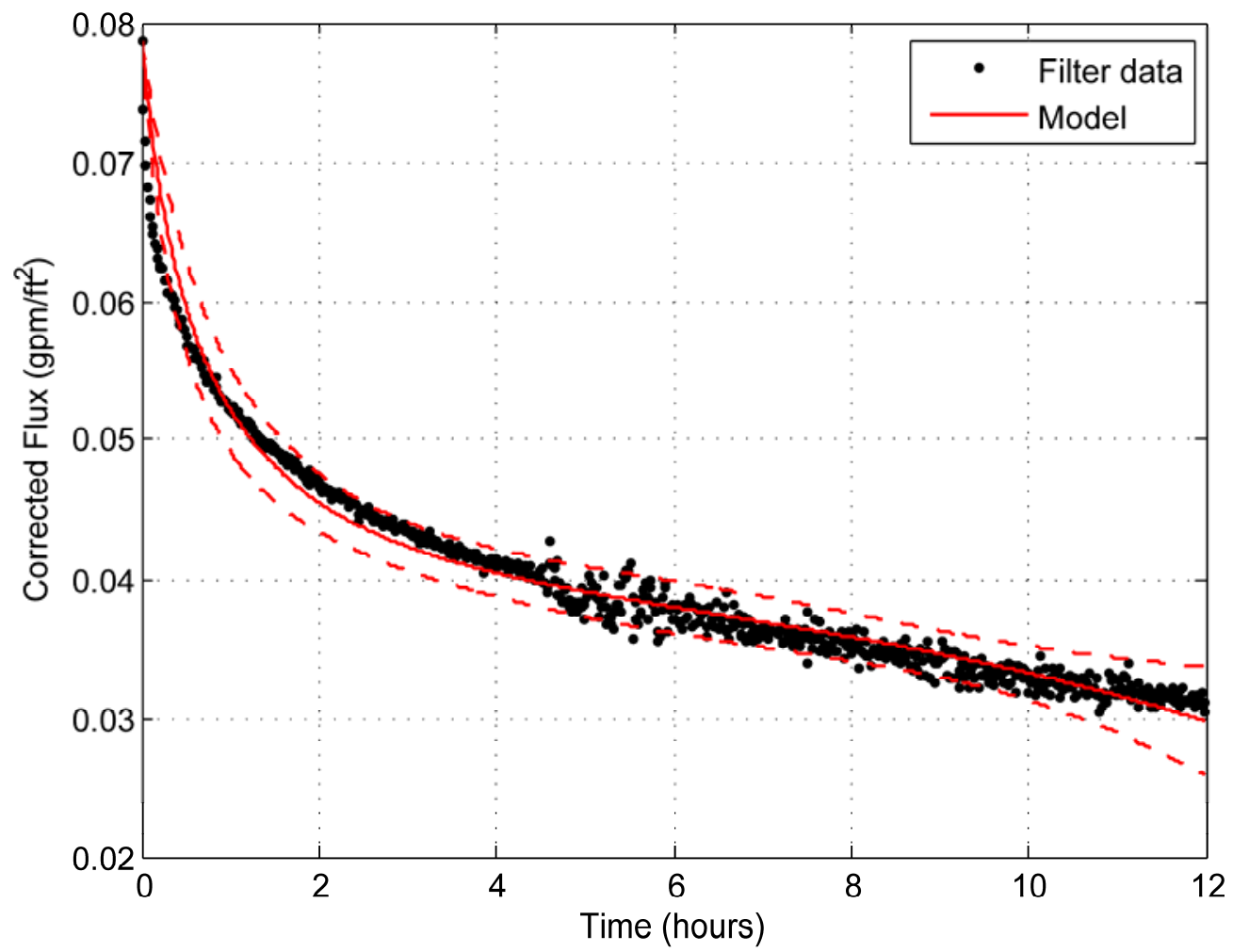

Figure 8.3. Original Model Fit to the First 12 Hours of Filter Data from S1T1. The dotted lines are the bands of $95 \%$ confidence for the model fit.

A simple modification to the model of Equations $8.5-8.8$ was proposed to give physically reasonable behavior of the resistances, i.e., to observe increasing $R_{c}$ with time as suggested by value of $K_{c}$ $<1$. The modification was as follows:

$$
\begin{aligned}
& \frac{d R_{m}^{*}}{d t^{*}}=\beta J^{*} R_{m}^{* 2} \text { when } t^{*} \leq t_{c r}^{*}, \\
& \frac{d R_{m}^{*}}{d t^{*}}=0 \text { when } t^{*}>t_{c r}{ }^{*} .
\end{aligned}
$$

The critical time $t_{c r}{ }^{*}$ was arbitrarily chosen to occur when $R_{m}{ }^{*}=R_{c}{ }^{*}$, with the assumption that the membrane fouling mechanism "shuts off" once the cake resistance becomes comparable in magnitude. The critical time was solved for iteratively, and an example of the resistance behavior that results is presented in Figure 8.5. Though the modification is more consistent with expectation, it did not improve the goodness of the fit (if anything, it resulted in a poorer fit) and has the undesirable result of adding a sharp "edge" to the $R_{m}{ }^{*}$ curve and flux model (Figure 8.5).

Interestingly, based on the parameters that minimize the residuals, the critical time was found to be a function of TMP, as a larger TMP resulted in a shorter critical time. Thus, the modification as written in Equation 8.9 predicts that the rate of growth of cake resistance depends on the TMP used during filtration. The membrane resistance can be used to predict the flux after backpulsing. However, the flux was not consistently predicted by the membrane resistance; the average error between the predicted and observed 
flux was approximately $16 \%$ (see Table 8.2 ). ${ }^{8}$ The error in prediction is not surprising, given that the criterion for implementing Equation 8.9 was arbitrary and the model does not predict the data well at short-times $(t<5$ hours $)$.

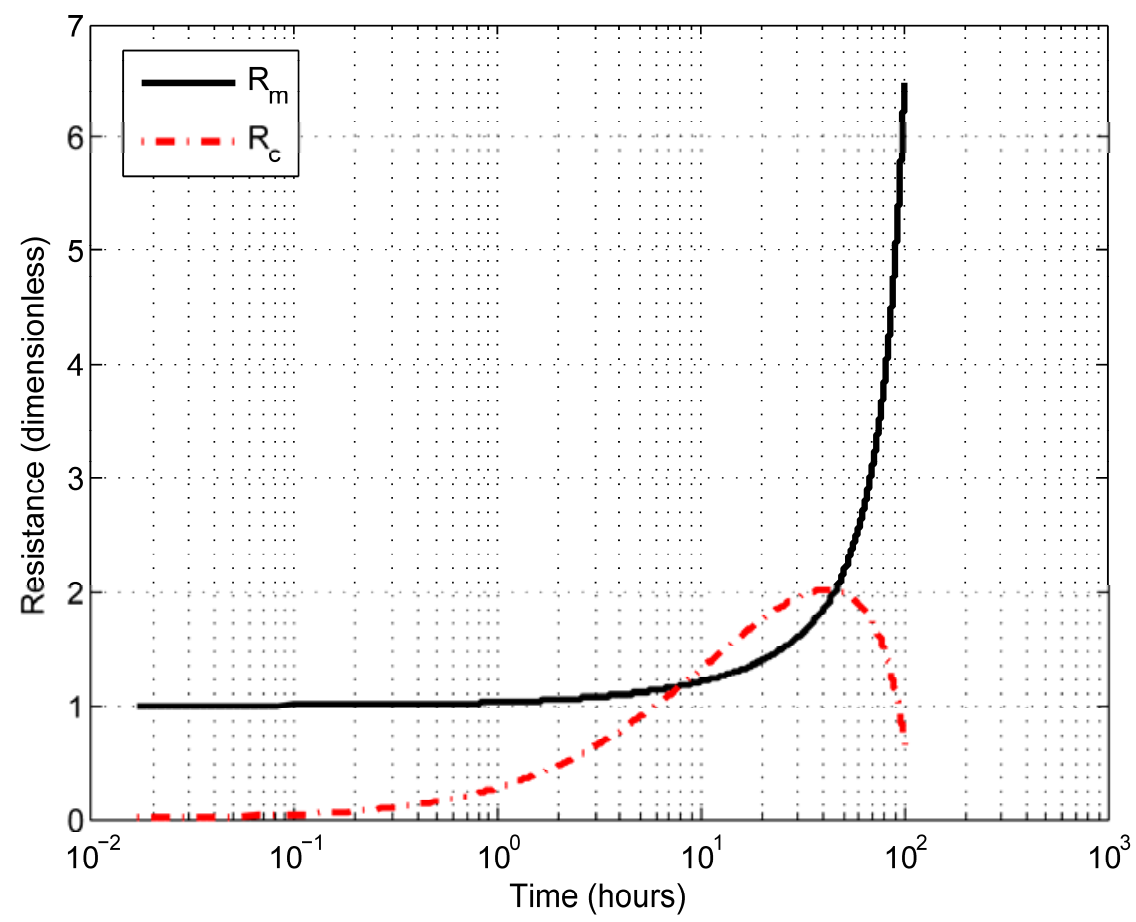

Figure 8.4. Dimensionless Membrane $\left(\mathrm{R}_{\mathrm{m}}\right)$ and Cake $\left(\mathrm{R}_{\mathrm{c}}\right)$ Resistance as a Function of Time for the Model Fit to S1T1 Data (see Figure 8.1)

Table 8.2. Results of the Non-Linear Least Squares Minimization Applied to the Series 1 Data Using the Criterion of Equation 8.9

\begin{tabular}{cccccc}
\hline Test & S1T1 & S1T2 & S1T3 & S1T4 & S1T5 \\
\hline$\beta$ & 0.1139 & 0.0789 & 0.1131 & 0.1582 & 0.0155 \\
$\mathrm{~K}_{\mathrm{c}}$ & 0.1222 & 0.1921 & 0.0879 & 0.0765 & 0.2916 \\
$\mathrm{k}_{\mathrm{m}}$ & $9.66 \times 10^{-14}$ & $7.06 \times 10^{-14}$ & $7.19 \times 10^{-14}$ & $1.68 \times 10^{-13}$ & $2.10 \times 10^{-14}$ \\
$\mathrm{k}_{\mathrm{c} 1}\left(\mathrm{~m}^{-2}\right)$ & $2.78 \times 10^{12}$ & $2.49 \times 10^{12}$ & $3.21 \times 10^{12}$ & $5.35 \times 10^{12}$ & $1.63 \times 10^{12}$ \\
$\mathrm{k}_{\mathrm{c} 2}\left(\mathrm{~m}^{-1} \mathrm{~s}^{-1}\right)$ & $1.80 \times 10^{7}$ & $2.64 \times 10^{7}$ & $1.20 \times 10^{7}$ & $2.51 \times 10^{7}$ & $1.84 \times 10^{7}$ \\
$t_{c r}($ hours $)$ & 9.3 & 10.7 & 10.9 & 5.8 & 17.2 \\
$J_{b p}\left(\mathrm{~m} \mathrm{~s}^{-1}\right)$, pred & $4.39 \times 10^{-5}$ & $4.79 \times 10^{-5}$ & $3.62 \times 10^{-5}$ & $4.67 \times 10^{-5}$ & $3.76 \times 10^{-5}$ \\
$J_{b p}\left(\mathrm{~m} \mathrm{~s}^{-1}\right)$, obs & $3.82 \times 10^{-5}$ & $3.74 \times 10^{-5}$ & $4.33 \times 10^{-5}$ & $5.07 \times 10^{-5}$ & $3.31 \times 10^{-5}$ \\
$\mathrm{SSE}^{(a)}$ & 3.742 & 4.895 & 2.713 & 3.397 & 4.502 \\
$\mathrm{MSE}^{(\mathrm{b})}$ & $6.18 \times 10^{-4}$ & $8.15 \times 10^{-4}$ & $4.53 \times 10^{-4}$ & $5.65 \times 10^{-4}$ & $7.53 \times 10^{-4}$ \\
\hline
\end{tabular}

(a) Sum of squared errors of prediction.

(b) Mean squared error.

8 The error ranged from an overprediction of $28 \%$ (S1T2) to an underprediction of $16 \%$ (S1T3). 


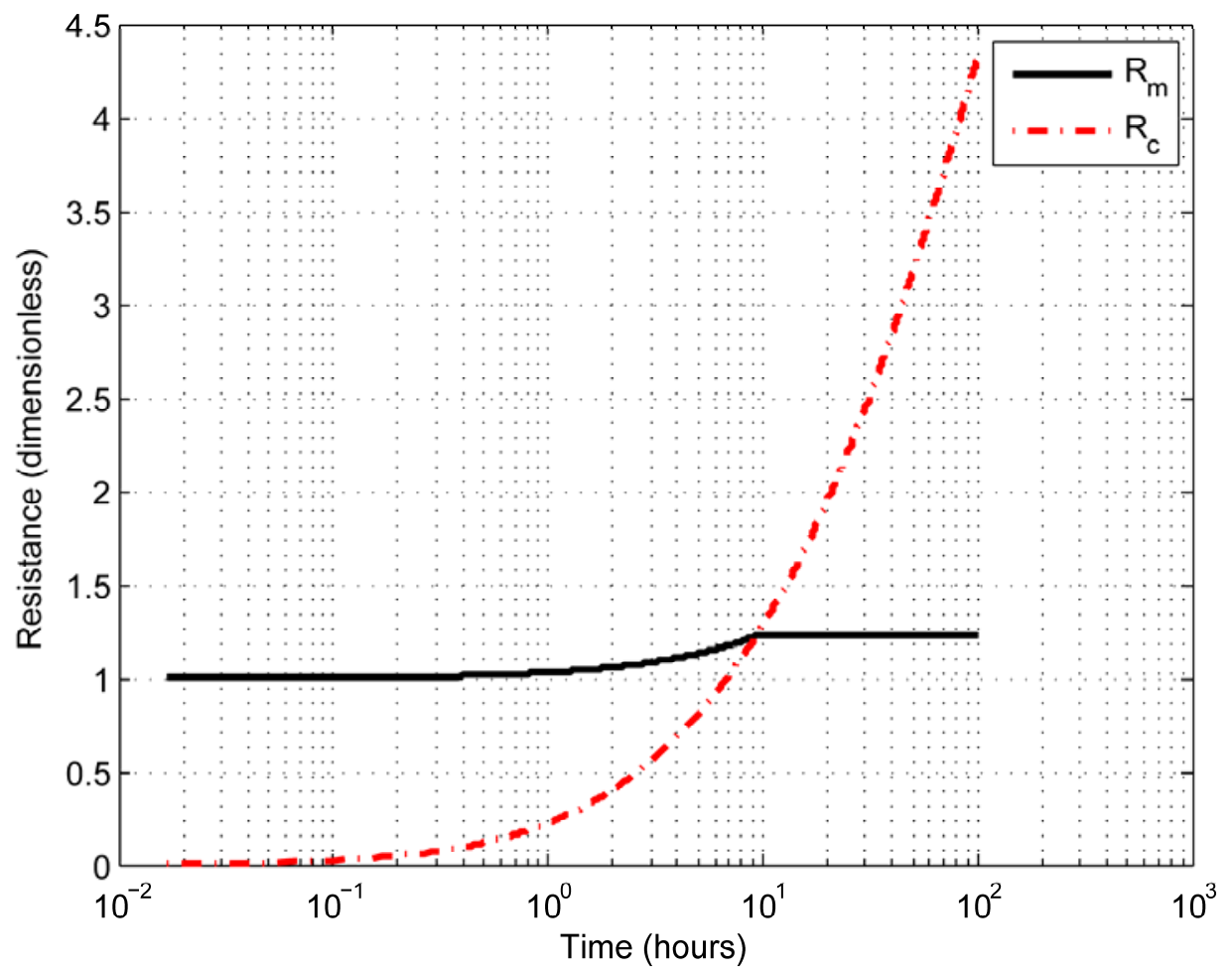

Figure 8.5. Dimensionless Membrane $\left(\mathrm{R}_{\mathrm{m}}\right)$ and Cake $\left(\mathrm{R}_{\mathrm{c}}\right)$ Resistance as a Function of Time for the Modified Model Fit to S1T1 Data

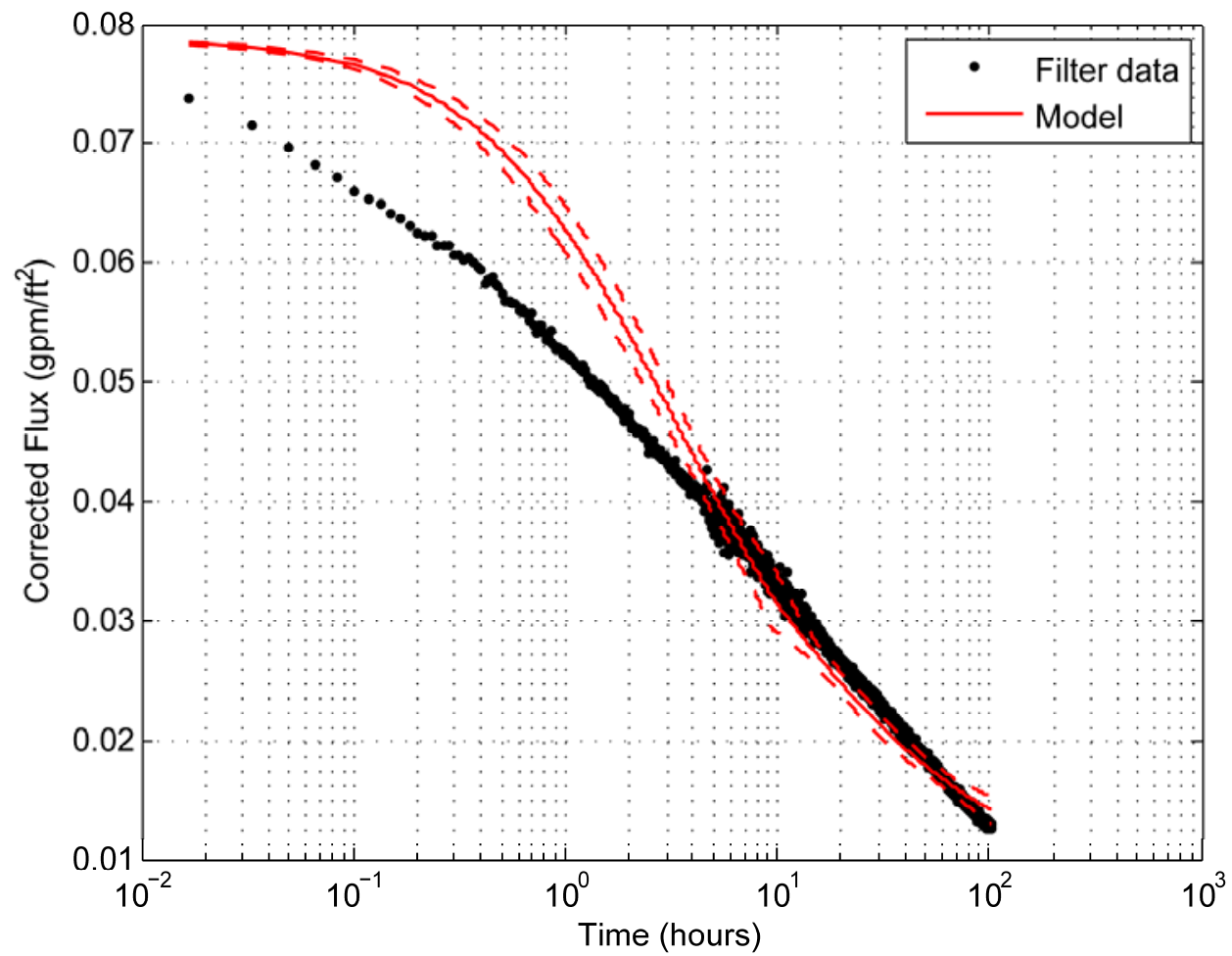

Figure 8.6. Modified Model Fit to S1T1 Data Before Backpulsing ( $\sim 100$ hours) Using Constraint in Equation 8.9. The dotted lines are the $95 \%$ confidence bands for the model fit. 
Another modification to the model of Equations $8.5-8.8$ that was considered was to constrain the model using the critical time formulation captured by Equation 8.9 and include the flux data observed after the backpulsing was performed. Including the post-backpulse data can be handled by assuming that all resistance due to the cake is reversible, and all resistance due to the membrane is irreversible.

Therefore, $R_{c}{ }^{*}$ is set equal to zero when filtering resumes after the backpulse but the value of $R_{m}{ }^{*}$ is retained. When this approach is used, the model fits the post-backpulse data adequately. This comes at the expense of the pre-backpulse data, which is described poorly by the modified model (see Figure 8.7). It is clear this modified model would not be a good predictor of long-term filtration performance and so further modifications were not pursued. It is interesting note that both of the modified models support the same relationship between $1 / K_{c}$ and $\Lambda$ as the original model did (see Figure 8.8). The figures resulting from the same analysis of tests S1T2 - S1T5 are presented in Appendix A.

The existing PNNL model, or any subsequent modification, did not describe the Series 1 flux data very well over the entire 100 hours, especially at shorter elapsed times. However, the model did indicate that cake formation is an important mechanism in this filtration system. Based on this observation, the approach was shifted to seek a mathematical model that had a mechanistic explanation for observed filtration behavior.

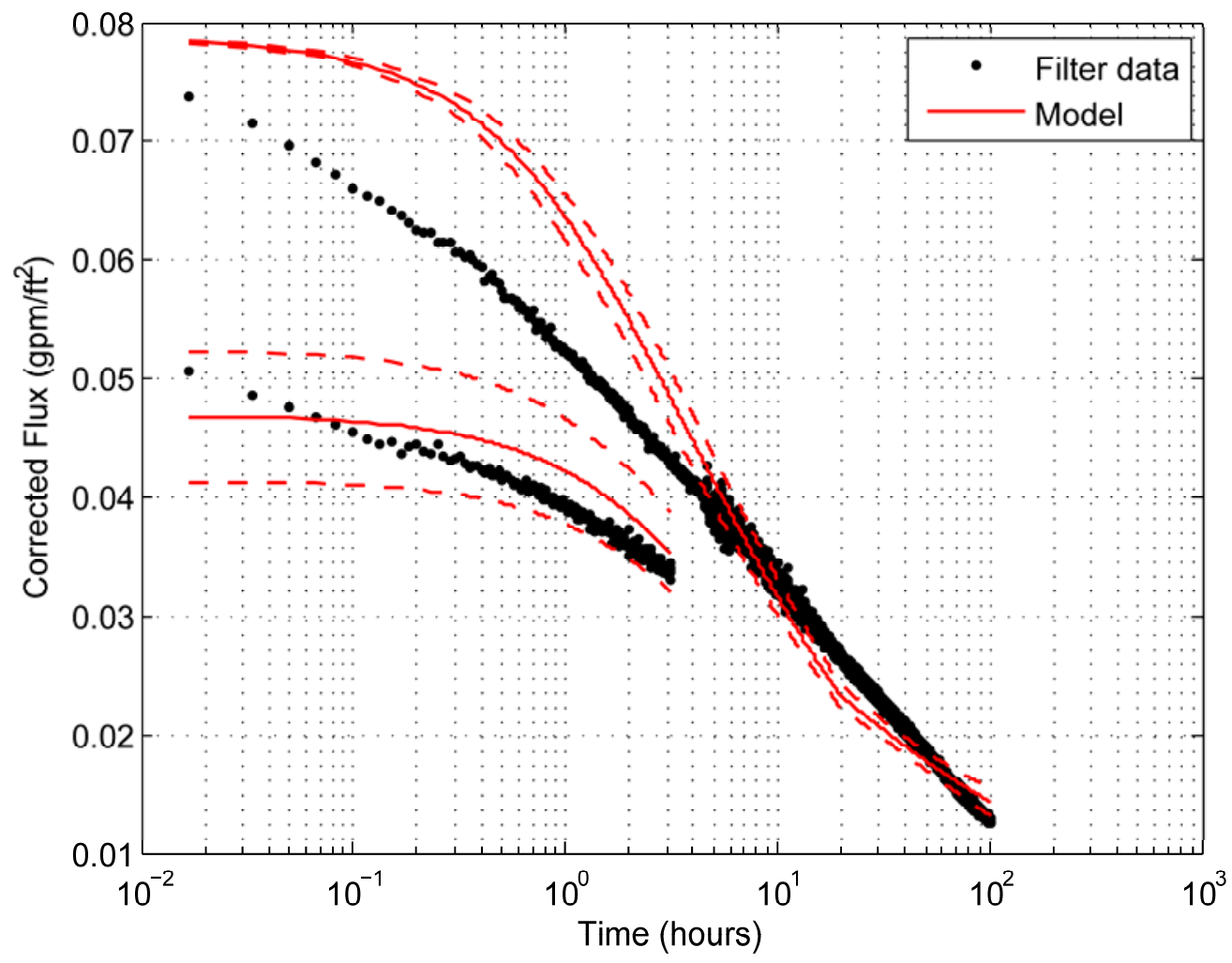

Figure 8.7. Modified Model Fit to S1T1 Data Before Backpulsing and After Backpulsing Using Constraint in Equation 8.9. After backpulsing data is shown as beginning again at $t=0$ for clarity of presentation. The dotted lines are the bands of $95 \%$ confidence for the model fit. 


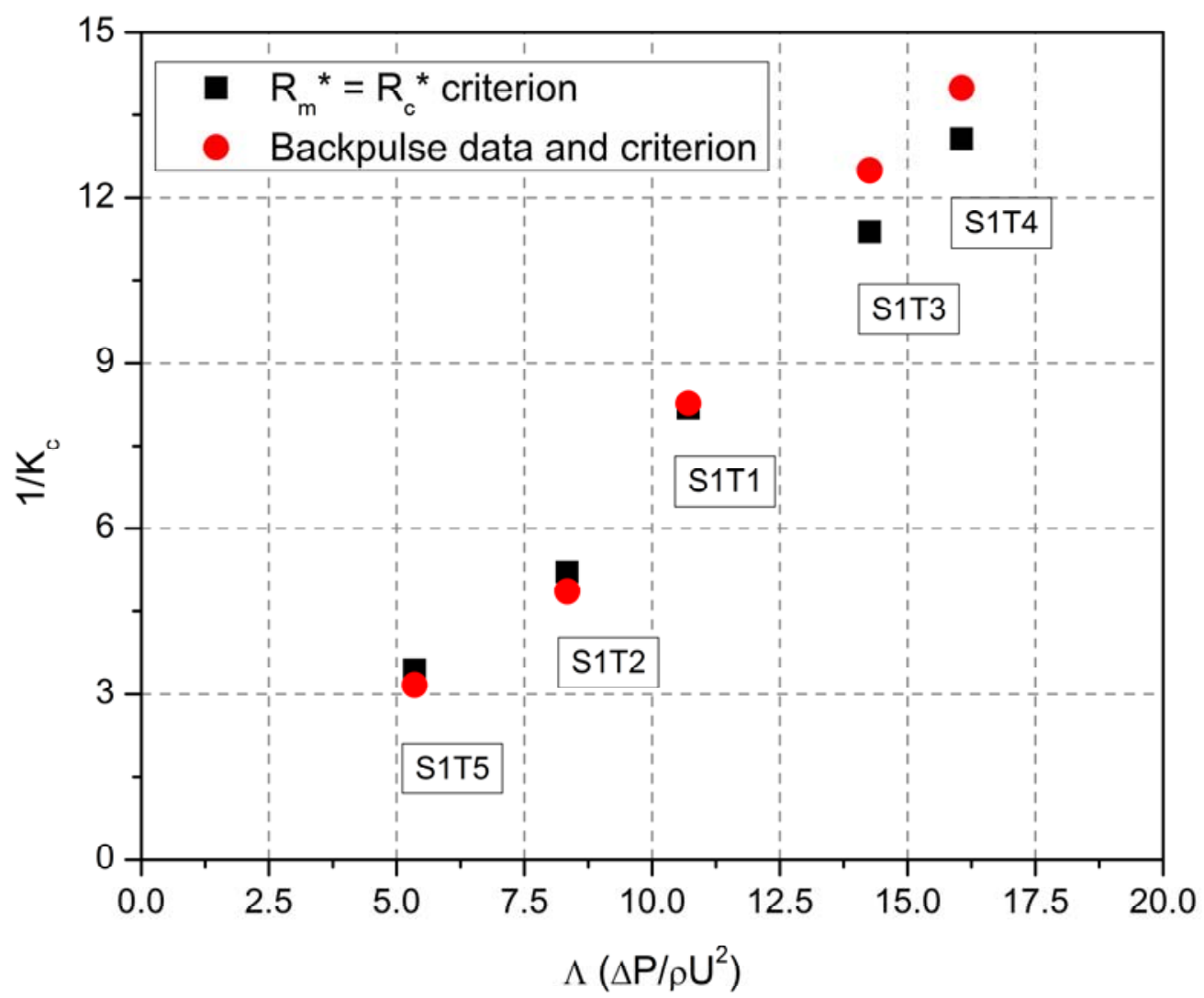

Figure 8.8. Cake Equilibrium Constant as Estimated Using the Constraint in Equation 8.9 (black squares) and Using the Backpulse Data with the Same Constraint (red circles) vs. Dimensionless Pressure Ratio $\Lambda$ for all Series 1 Tests

\subsection{Update to Filtration Model}

The results described in Section 8.1 demonstrated that the existing PNNL model fit a 12-hour segment of flux data well, but could not be extended to describe the filter fouling dynamics over the entire 100 hours of operation. Additional analysis was performed on the Series 1 filtration data, with the goal of updating the existing model. The model described in Section 8.1 assumes that two resistances are present ( $R_{m}$, an irreversible membrane resistance, and $R_{c}$, a reversible cake resistance) but does not specify the mechanism(s) that affect the resistances over time. Rather than attempt to fit the entire range of data using a resistance model, the focus was shifted to identifying the long-time mechanism(s) and the approximate points in time where they dominate the filtration behavior.

The four most common fouling mechanisms are well described in the literature (see, for example Hermia (1981) or the summary in Daniel et al. [2010a]). The first attempt to understand the long-time fouling mechanism was to test each of the four most common fouling mechanisms (complete, standard, cake, and intermediate) against the long-time data ( $t>10$ hours elapsed time) of the five Series 1 tests. The data for $t>10$ hours was chosen because visual inspection of the flux data suggests that the change of the flux with time is approximately constant from 10 hours onward. 
The fouling mechanisms each describe a different process by which the filter experiences reductions in permeate flux. Complete blocking assumes that particles completely seal off pores, thereby reducing the pore cross-sectional area available for permeate removal. Standard blocking assumes that particles accumulate on the walls of pores, constricting the pore area and increasing membrane resistance. Cake fouling assumes that a layer of particles (which may increase in thickness with time) assembles on the filter surface. The cake is permeable to flow but adds additional hydrodynamic resistance to permeate flow. Finally, intermediate blocking assumes some pores are sealed by a fraction of the particles (as in complete blocking) and then other particles accumulate on top of these blocking particles.

For ease of comparison, the common mathematical model for the fouling models was linearized. The general differential form describing the filtration "blocking laws" is

$$
\frac{d^{2} t}{d V^{2}}=k\left(\frac{d t}{d V}\right)^{n}
$$

where $t$ is the filtration time, $V$ is the total filtered (permeate) volume, and $J=1 / A_{F} d V / d t$. The exponent $n$ determines the choice of fouling mechanism, as represented by a blocking law: $n=0$ for cake filtration, $n=1$ for intermediate blocking, $n=3 / 2$ for standard blocking (sometimes called pore constriction, hereafter referred to as constriction blocking), and $n=2$ for complete pore blocking (referred to hereafter as pore blocking). Hermia derives linearized forms for these four blocking laws:

Cake $(\mathrm{n}=0)$ :

$$
K_{c} V=\frac{1}{Q}-\frac{1}{Q_{o}}
$$

Intermediate $(\mathrm{n}=1)$ :

$$
Q=Q_{o} e^{-K_{i} V}
$$

Constriction $(\mathrm{n}=3 / 2)$ :

$$
Q=Q_{o}\left(1-\frac{K_{s} V}{2}\right)^{2} ; \text { and }
$$

Pore $(n=2)$ :

$$
K_{b} V=Q_{o}-Q
$$

In Equations 8.11 through 8.14, $Q$ is the volumetric flow rate of permeate at time $t, Q_{o}$ is the initial volumetric flow rate, and $K_{c}, K_{i}, K_{s}$, and $K_{b}$ are fouling constants. After some algebraic rearrangement and dividing through by the constant filter area $A_{F}$, the following volume-flux expressions are obtained:

$$
\begin{aligned}
& \text { Cake }(\mathrm{n}=0): \quad V=\frac{1}{A_{F} K_{c}}\left(\frac{A_{F}}{Q}-\frac{A_{F}}{Q_{o}}\right)=K_{c}^{*}\left(\frac{1}{J}-\frac{1}{J_{o}}\right) ; \\
& \text { Intermediate }(\mathrm{n}=1):
\end{aligned}
$$

Constriction $(\mathrm{n}=3 / 2): \quad\left(\frac{Q}{Q_{o}}\right)^{1 / 2}=1-\frac{K_{s} V}{2} \Rightarrow V=\frac{2}{K_{s}}\left(J_{o}^{1 / 2}-J^{1 / 2}\right)$; and 
Pore $(\mathrm{n}=2)$ : $\quad V=K_{b}^{*}\left(J_{o}-J\right)$.

The new constants $K_{c}{ }^{*}$ and $K_{b}{ }^{*}$ have incorporated the filter area into them. By examining Equations 8.15 through 8.18, it was expected that the $\mathbf{f}(J)$ representing a particular blocking law would exhibit a linear relationship with $V$ if any one fouling mechanisms is dominating the filtration behavior. For the cake, intermediate, constriction and pore mechanisms, $\mathbf{f}(J)$ is $J^{-1}, \ln J, J^{1 / 2}$, and $J$, respectively. Since only the $t>10$ hours flux data was used, it was not expected that either $J_{o}$ or the specific blocking constant would be estimated accurately; thus, they were not included in the analysis.

An example of the linearized plots generated using Equations 8.15 through 8.18 is shown in Figure 8.9 for the data of test S1T1. For each mechanism, the appropriate $\mathbf{f}(J)$ was calculated for data greater than 10 hours. The data was tested for linearity by applying a linear fit to the data and calculating some goodness-of-fit statistics. The linear fit was also applied at other time intervals $(t>25$ and $t>50$ hours) to assess the changes in slope, if any. Observed changes in slope indicate that the linear fit is not appropriate over the entire data range; thus, the mechanism exhibiting slope changes is probably not controlling long-time fouling behavior. After examination of Figure 8.9, it can be concluded that the pore and constriction mechanisms do not appear to be the cause of long-time fouling. The intermediate mechanism looks to be the best choice, particularly over the entire data range. The goodness-of-fit statistics reflect this (see Figure 8.10 for an example).

The linearized mechanism comparisons were repeated for each Series 1 test, with the same conclusion (see Appendix A). It was found that the intermediate mechanism was the best long-time fit to the data. However, note from Figure 8.9 that all four blocking law plots have some curvature as the time approaches 10 hours, i.e., as the data moves toward the left-hand side of the plot. To further examine the data, the same approach was repeated for intermediate times $(5<t<20$ hours of elapsed time). The linearized plots generated for this period are shown in Figure 8.11, and the goodness-of-fit comparison is given in Figure 8.12.

None of the mechanisms fit the data well if data between $5<t<10$ hours is considered. This may suggest that some other mechanism is taking place at short-times that is not well described by any of the four blocking laws. The cake mechanism is the best fit, especially as the time period lengthens beyond the $5<t<10$ hours data set. Using this approach, which has a coarse time resolution, the distinction between the cake and intermediate mechanisms is subtle, as either does a passable job of fitting the entire range ( $t>5$ hours elapsed time) of data.

A transition between cake formation and an intermediate mechanism is conceivable. Consider the following narrative, in the context of filtration behavior over time in Series 1 testing. At short-times, the initially clean membrane is fouled by some of the initial particles that come into proximity with the pores. This continues to occur but the process slows down as the cake begins to assemble, and later, at intermediate times, to mature. The maturation of the cake could occur through either increased thickness or decreased porosity (via compaction or incorporation of fines into the cake). Eventually the cake maturation will become physically limited, either by growing to a thickness in equilibrium with the bulk turbulent flow, reaching a maximum particle packing in the cake, or both. The intermediate mechanism is, in physical terms, an acknowledgement that particle interactions with the filter are not always as simple as a single particle completely blocking a pore or participating in a cake. Thus, it describes particle interactions where particles can settle on other particles in a pore, i.e., a sub-surface cake. Once 

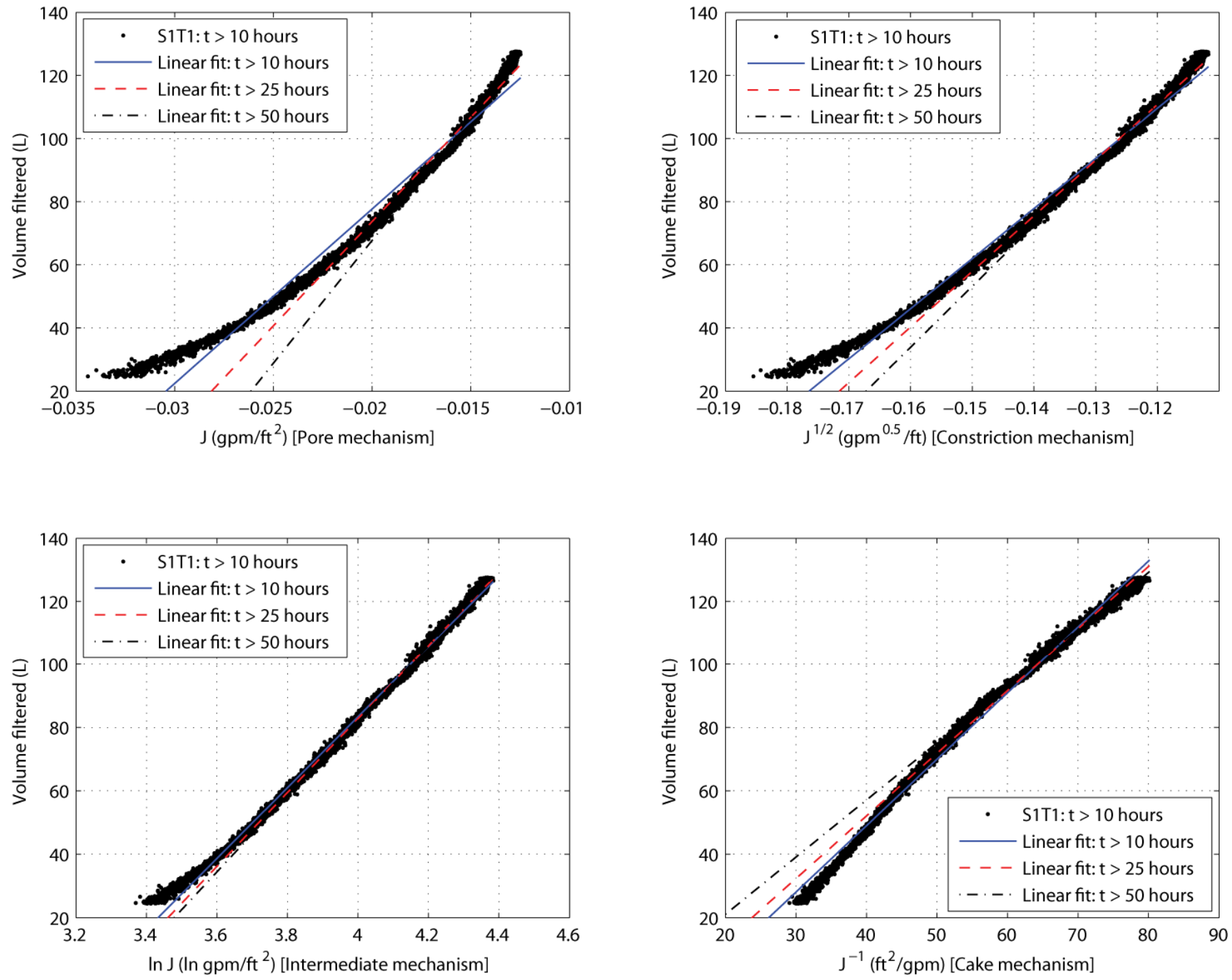

Figure 8.9. Linearized Blocking Laws for S1T1 at Long-Times: $f(J)$ Versus $V$, with Linear Regression Applied for Data from $t>10, t>25$, and $\mathrm{t}>50$ Hours. Clockwise from top left: pore, constriction, cake and intermediate blocking laws, respectively. 


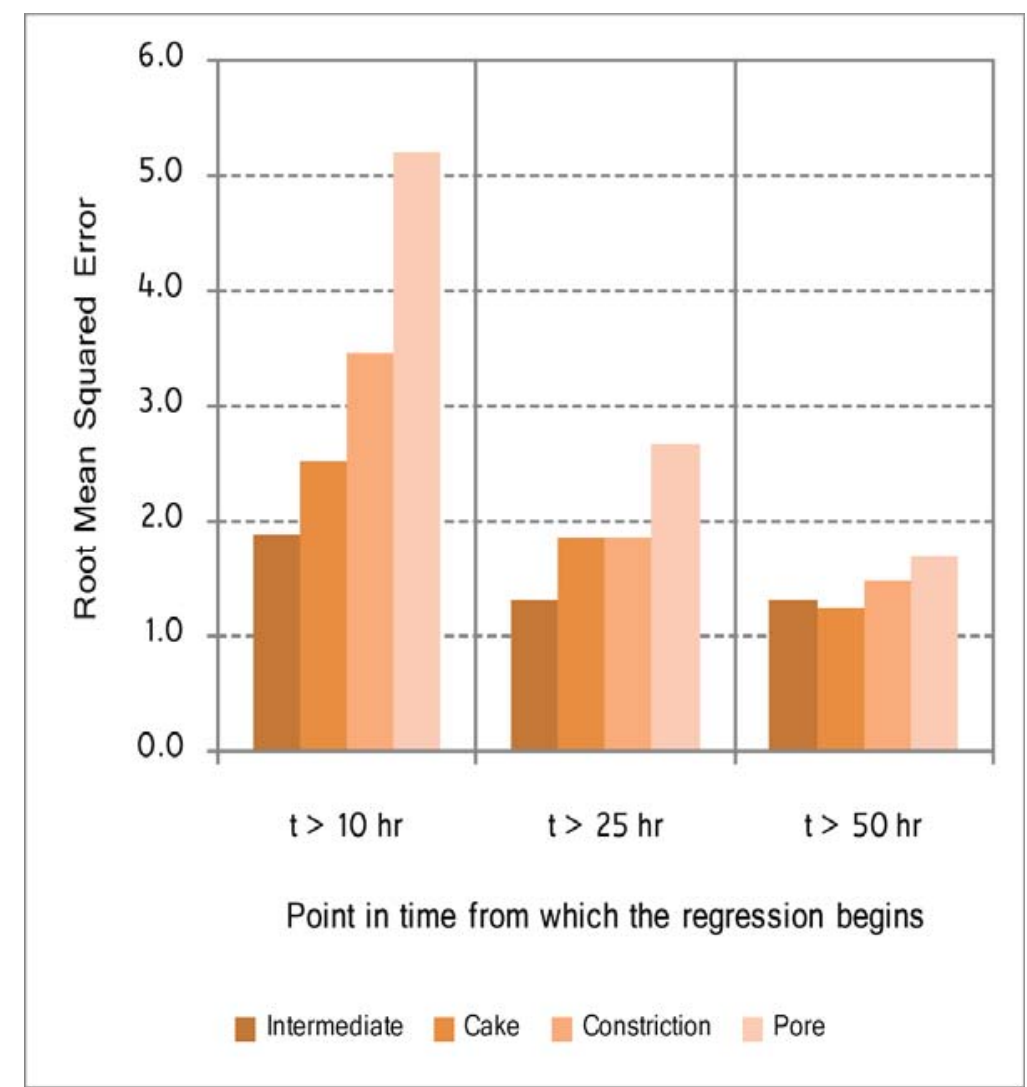

Figure 8.10. The Root-Mean Squared Error for the Regression Performed Using the Four Linearized Mechanistic Models on S1T1 Flux Data for Long-Times

particles can no longer participate in the cake, they accumulate in the filter pores instead. The exact mechanism for this is not clear, but it could be speculated that the surface cake loses particles over time or non-cake particles slowly migrate down to the pores. Assuming for a moment this narrative is representative of what actually occurs during filtration, it is consistent with expectation that the formation of a sub-surface cake would occur slowly.

In order to distinguish between the two mechanisms, a more rigorous fitting routine was applied to the Series 1 data. For convenience, instead of using the expressions in Equations 8.15 and 8.16, a flux-time construction was used (for example, see Peng and Tremblay (2008), Table 3). The intermediate mechanism model, which is assumed to apply at some as-of-yet unknown long-time, is written as

$$
\frac{J}{J_{o}}=\frac{1}{1+a_{\mathrm{int}} t}
$$

where $a_{\text {int }}$ is the intermediate constant with units hour ${ }^{-1}$. Similarly, the cake mechanism model is assumed to apply at some unknown intermediate time, and is written as

$$
\frac{J}{J_{o}}=\frac{1}{\left(1+a_{\text {cake }} t\right)^{1 / 2}}
$$

where $a_{\text {cake }}$ is the cake constant with units hour ${ }^{-1}$. 

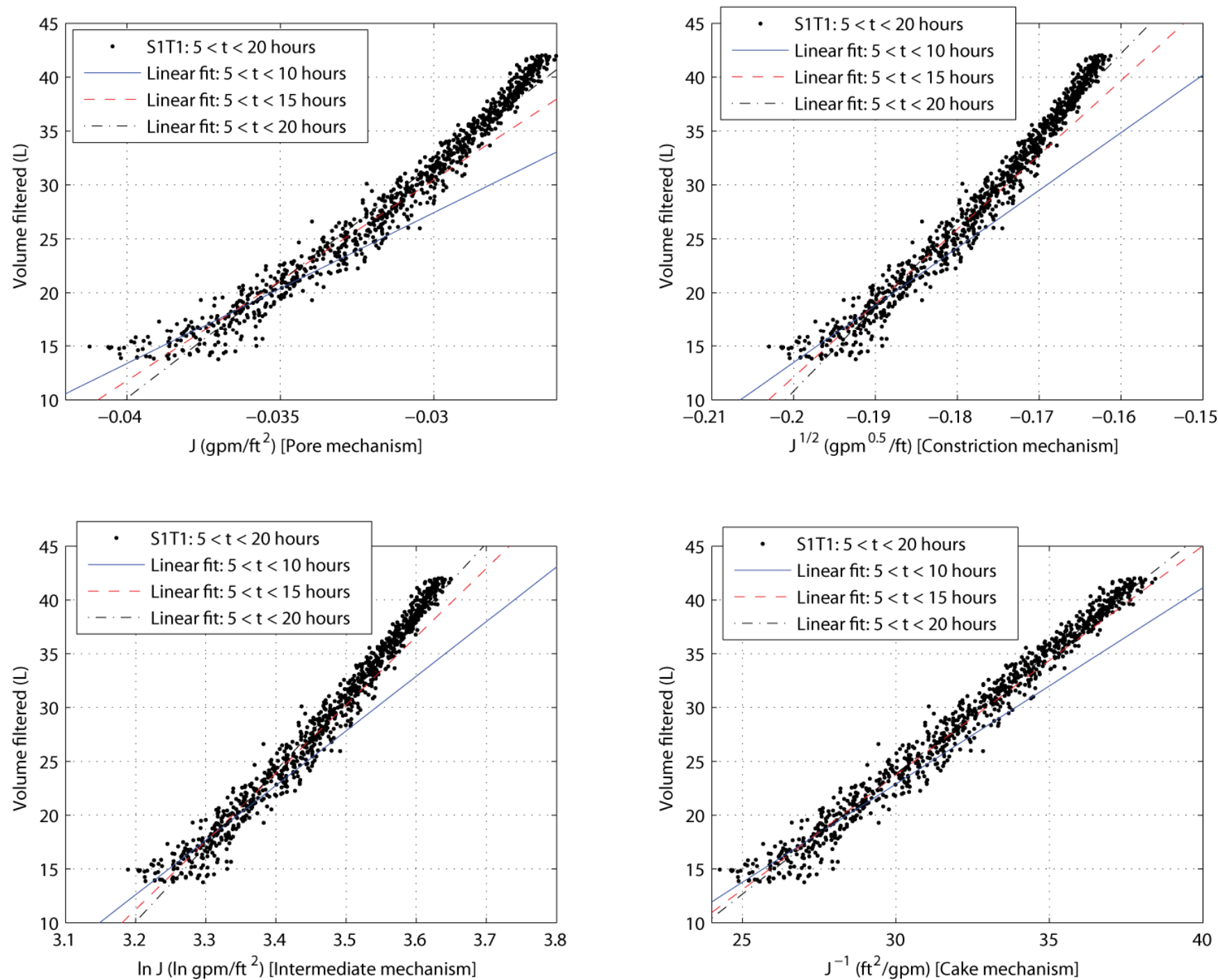

Figure 8.11. Linearized Blocking Laws for S1T1 at Intermediate Times: $\mathrm{f}(J)$ Versus $V$, with Linear Regression Applied for Data from $5<t<10,5<t<15$, and $5<t<20$ Hours. Clockwise from top left: pore, constriction, cake and intermediate blocking laws, respectively. 


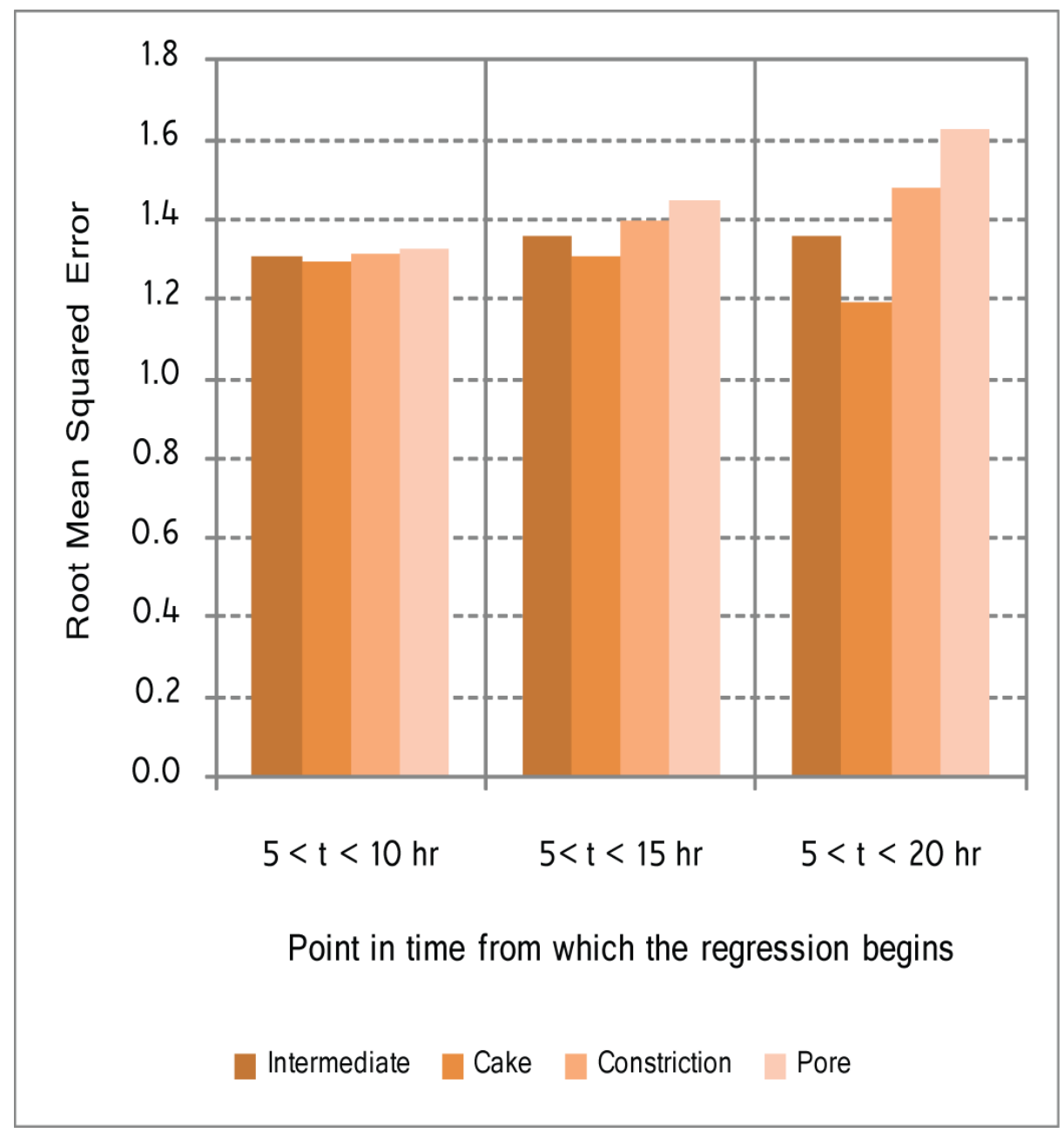

Figure 8.12. The Root-Mean Squared Error for the Regression Performed Using the Four Linearized Mechanistic Models on S1T1 Flux Data for Intermediate Times

There was concern that modeling the flux scaled to $J_{o}$ when the fouling phenomena occur on a long-time scale (and well after $t=0$ ) would not be appropriate. In addition to this concern, the value of the initial flux is highly dependent on the state of the filter (e.g., cleaning effectiveness, fouling occurring before the test begins) and the startup conditions (e.g., rate of TMP or AV increase, time to target TMP or AV). Thus, instead of using Equation 8.19 as written, it was recast. Assuming that the intermediate mechanism model is valid at some time $t=t_{1}$, then

$$
\frac{J_{1}}{J_{o}}=\frac{1}{1+a_{\mathrm{int}} t_{1}}
$$

where $J_{1}$ is the filter flux at $t_{1}$. If a shifted time $\tau_{1}=t-t_{1}$ is defined, Equation 8.19 can also be written as

$$
\frac{J}{J_{o}}=\frac{1}{1+a_{\mathrm{int}} t_{1}+a_{\mathrm{int}} \tau_{1}} .
$$


Combining Equations 8.21 and 8.22, then rearranging the expression yields

$$
\frac{J}{J_{1}}=\frac{\left(1+a_{\mathrm{int}} t_{1}\right)}{\left(1+a_{\mathrm{int}} t_{1}\right)+a_{\mathrm{int}} \tau_{1}}=\frac{1}{1+\left(\frac{a_{\mathrm{int}}}{1+a_{\mathrm{int}} t_{1}}\right) \tau_{1}} ;
$$

Note that Equation 8.23 is now independent of the initial flux and shifts the time variable to begin at zero when $J=J_{1}$. Making the further substitution of a modified constant defined as

$$
a_{1}=\frac{a_{\text {int }}}{1+a_{\text {int }} t_{1}}
$$

allows a simpler expression to be used when conducting the computational fitting $\left(a_{\text {int }}\right.$ can be calculated by rearranging Equation 8.24). The initial flux can be projected from the results of the fit via the simple relationship $J_{o, 1}=J_{1}\left(1+a_{\text {int }} t_{1}\right)$. The projected flux implies what the filter performance would be at $t=0$ if the intermediate mechanism was controlling flux behavior over the entire time of operation.

A similar manipulation of the cake mechanism model in Equation 8.20 yields the final expression

$$
\frac{J}{J_{2}}=\frac{\left(1+a_{\text {cake }} t_{2}\right)^{1 / 2}}{\left(\left(1+a_{\text {cake }} t_{2}\right)+a_{\text {cake }} \tau_{2}\right)^{1 / 2}}=\frac{1}{\left(1+\left(\frac{a_{\text {cake }}}{1+a_{\text {cake }} t_{2}}\right) \tau_{2}\right)^{1 / 2}},
$$

where $J_{2}$ is the filter flux at time $t_{2}$, the shifted time $\tau_{2}=t-t_{2}$, and $t_{2}<t_{1}$. The same substitution of a modified constant was made, i.e.,

$$
a_{2}=\frac{a_{\text {cake }}}{1+a_{\text {cake }} t_{2}}
$$

The cake constant $a_{\text {cake }}$ can be calculated by rearranging Equations 8.26 and the projected flux is defined as $J_{0,2}=J_{2}\left(1+a_{\text {cake }} t_{2}\right)^{1 / 2}$.

The intermediate mechanism model given by Equation 8.23 was fit to flux data at long-times. The initial time $t_{1}$ was varied in half-hour increments until an optimum fit was found. The optimum was identified using goodness-of-fit statistics, such as a minimum in the root-mean squared error or a maximum in the $\mathrm{R}^{2}$ value. An example of an optimum fit obtained using this approach is presented in Figure 8.13 ; the fit is excellent, especially considering the model has only one adjustable parameter. The residuals were checked with a one-sample t-test at the $95 \%$ confidence level that they were randomly distributed with a mean of zero. They were also examined on a normal probability plot to look for bias or outliers. The full results of the analysis are given in Table 8.3, and an example of plots of the goodness-of-fit statistics and probability plots (for the S1T1 data) are supplied in Appendix A. Also included in Appendix A are the optimum fit plots for tests S1T2 - S1T5. 
Table 8.3. Results Obtained from Optimum Intermediate Mechanism Model of Series 1 Flux-Time Data

\begin{tabular}{cccccc}
\hline Test & S1T1 & S1T2 & S1T3 & S1T4 & S1T5 \\
\hline$a_{1}\left(\mathrm{hr}^{-1}\right)$ & 0.0137 & 0.0108 & 0.0159 & 0.0180 & 0.0088 \\
$a_{\text {int }}\left(\mathrm{hr}^{-1}\right)$ & 0.0188 & 0.0139 & 0.0219 & 0.0253 & 0.0106 \\
$J_{1}\left(\mathrm{~m} \mathrm{~s}^{-1}\right)$ & $1.79 \times 10^{-5}$ & $2.02 \times 10^{-5}$ & $1.61 \times 10^{-5}$ & $1.71 \times 10^{-5}$ & $1.87 \times 10^{-5}$ \\
$J_{\mathrm{o}, 1}\left(\mathrm{~m} \mathrm{~s}^{-1}\right)$ & $2.44 \times 10^{-5}$ & $2.62 \times 10^{-5}$ & $2.21 \times 10^{-5}$ & $2.40 \times 10^{-5}$ & $2.25 \times 10^{-5}$ \\
$t_{1}(\mathrm{hr})$ & 19.5 & 21.0 & 17.0 & 16.0 & 19.5 \\
$\mathrm{RMSE}^{(\mathrm{a})}$ & 0.0076 & 0.0057 & 0.0069 & 0.0099 & 0.0064 \\
$\mathrm{R}^{2(\mathrm{~b})}$ & 0.9973 & 0.9981 & 0.9980 & 0.9961 & 0.9969 \\
\hline
\end{tabular}

(a) Root-mean squared error at optimum $\left(t=t_{1}\right)$.

(b) Coefficient of variation at optimum $\left(t=t_{1}\right)$.

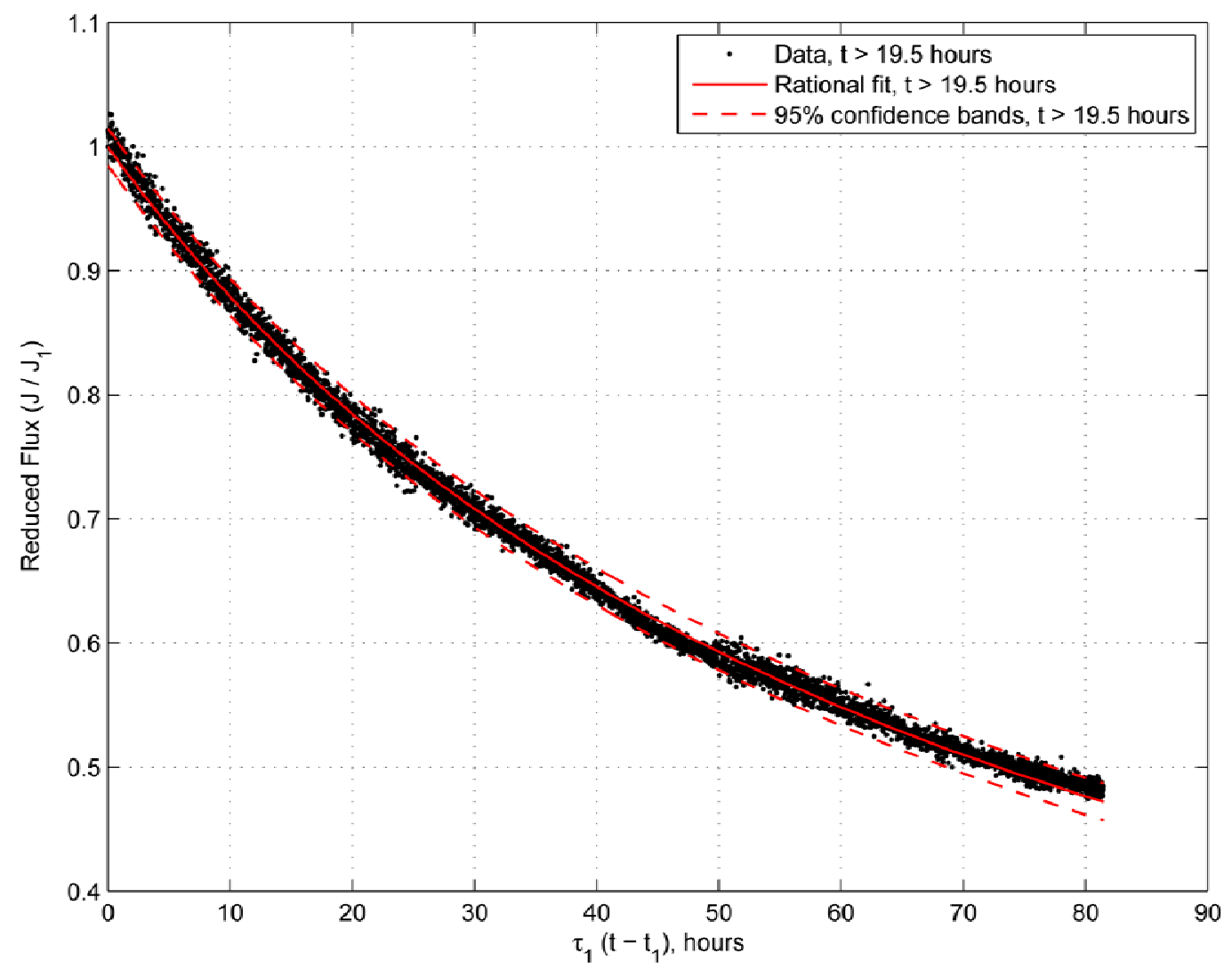

Figure 8.13. Optimum Fit to S1T1 Flux-Time Data Using the Model of Equation 8.23. The bands of $95 \%$ confidence for the fit are shown as dotted lines.

An example of the long-time optimum fit for S1T1 data projected back to the original flux-time data is shown in Figure 8.14. Note that the model describes the flux very well in the time range of interest but otherwise significantly underpredicts the flux. 


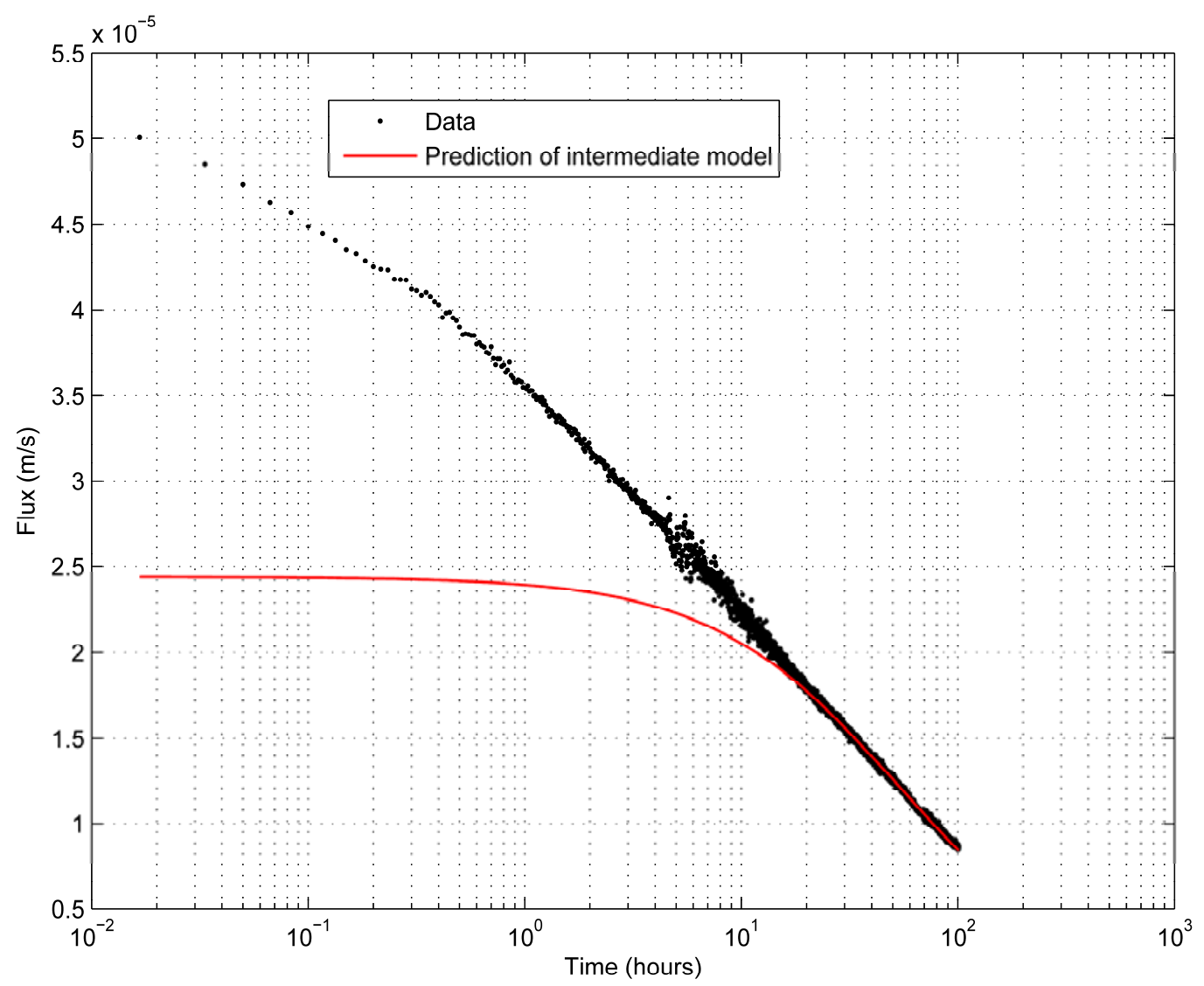

Figure 8.14. Long Time Optimum Fit for S1T1 Data Using the Intermediate Mechanism Model

The cake mechanism model given by Equation 8.25 was fit to flux data for an intermediate range of times, using the initial time from the optimum intermediate mechanism fit $\left(t_{1}\right)$ as the endpoint. The process was the same as for the intermediate mechanism fit, except that the initial time $t_{2}$ was varied in quarter-hour increments. An example of an optimum fit obtained using this approach is presented in Figure 8.15. Again, the fit is excellent and analysis of the residuals did not expose any areas of concern. The full results of the analysis are given in Table 8.4, and an example of plots of the goodness-of-fit statistics and probability plots (for the S1T1 data) are supplied in Appendix A. Also included in Appendix A are the optimum fit plots for tests S1T2 - S1T5.

The intermediate time optimum fit for S1T1 is presented in Figure 8.16. Note that the difference between the cake and intermediate mechanisms becomes more obvious from Figure 8.16. The cake mechanism, though it approximates the flux data at $t>t_{1}$, does not have the proper curvature past the endpoint of its applicability, e.g., approximately 19.5 hours. To confirm that a model that transitions from a cake-dominated resistance mechanism to an intermediate-dominated resistance mechanism was superior to one mechanism on its own, the cake and intermediate mechanisms were fit to the entire data range. Examination of the fits and the residuals illustrated that one mechanism alone does not properly describe the behavior of the flux with time. When applied over the entire data range, each of the mechanistic models have a trend in their residuals and they are rejected by the t-test at the $95 \%$ confidence level. 
Given that the filtration behavior after approximately 5 hours in all the Series 1 tests was controlled by cake and intermediate mechanisms, it was suspected that the rate at which the flux decays would depend on TMP and AV. Both the cake and intermediate mechanisms require that the solid particles in the slurry approach the filter surface, either to be incorporated into the cake or into a pore. The force driving particles to the filter surface (neglecting gravity) is TMP, whereas AV reduces the residence time of particles in the filter and re-suspends particles by shear. It was found that the characteristic constants of the intermediate and cake mechanisms models (each of which has only one adjustable parameter) have a linear relationship with the dimensionless quantity $\Lambda$ (recall this is transmembrane pressure/inertial pressure). The relationships are plotted in Figure 8.17. This relationship can be used to estimate filtration performance at elapsed times greater than 5 hours for a range of transmembrane pressures and axial velocities.

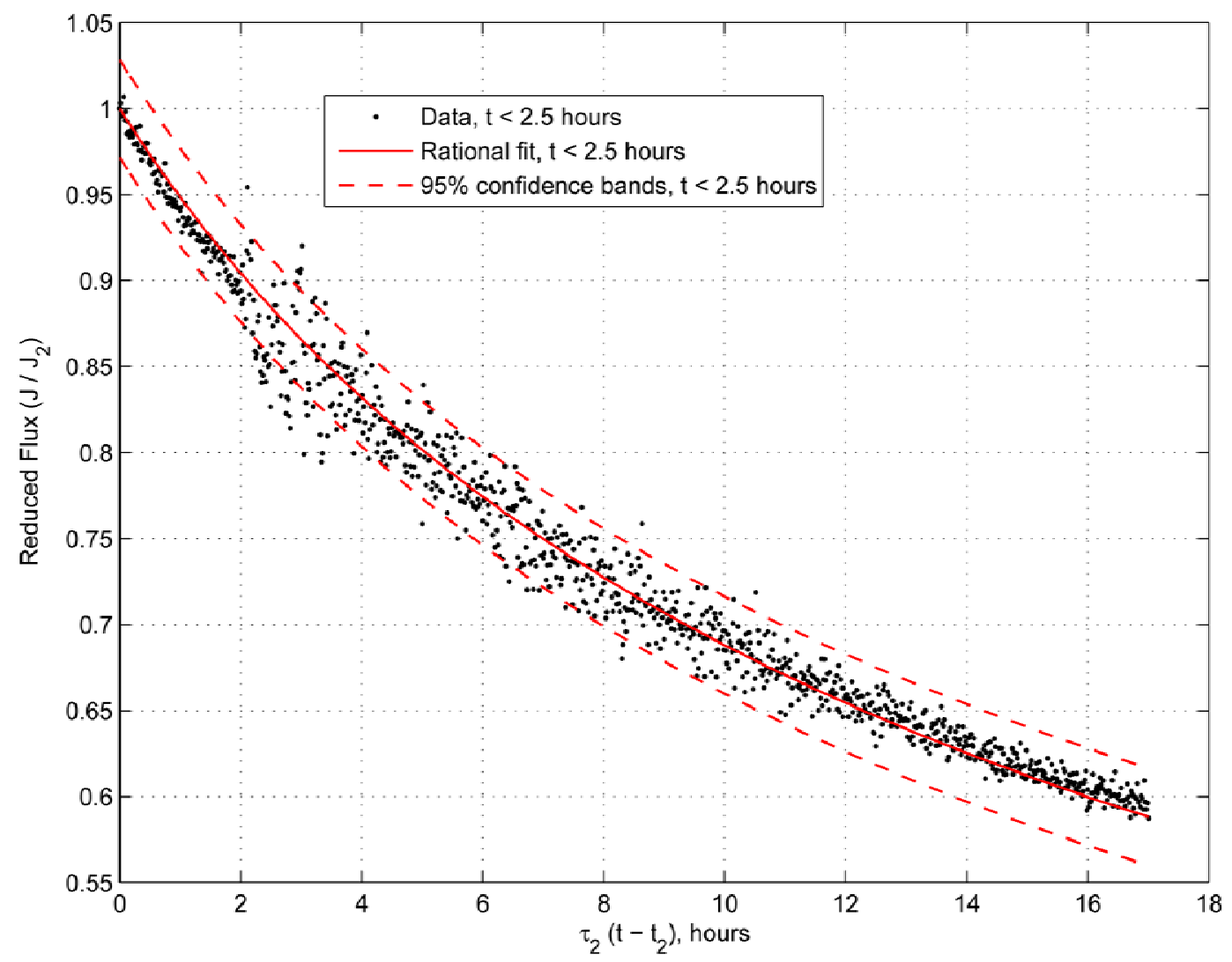

Figure 8.15. Optimum Fit to S1T1 Flux-Time Data Using the Model of Equation 8.25. The bands of $95 \%$ confidence for the fit are shown as dotted lines. 
Table 8.4. Results Obtained from Optimum Cake Mechanism Model of Series 1 Flux-Time Data

\begin{tabular}{cccccc}
\hline Test & S1T1 & S1T2 & S1T3 & S1T4 & S1T5 \\
\hline$a_{2}\left(\mathrm{hr}^{-1}\right)$ & 0.1113 & 0.0703 & 0.1150 & 0.1351 & 0.0411 \\
$a_{\text {cake }}\left(\mathrm{hr}^{-1}\right)$ & 0.1541 & 0.0932 & 0.1762 & 0.2273 & 0.0577 \\
$J_{2}\left(\mathrm{~m} \mathrm{~s}^{-1}\right)$ & $3.04 \times 10^{-5}$ & $3.04 \times 10^{-5}$ & $2.58 \times 10^{-5}$ & $2.84 \times 10^{-5}$ & $2.28 \times 10^{-5}$ \\
$J_{0,2}\left(\mathrm{~m} \mathrm{~s}^{-1}\right)$ & $3.58 \times 10^{-5}$ & $3.51 \times 10^{-5}$ & $3.19 \times 10^{-5}$ & $3.69 \times 10^{-5}$ & $2.70 \times 10^{-5}$ \\
$t_{2}(\mathrm{hr})$ & 2.5 & 3.5 & 3.0 & 3.0 & 7.0 \\
$\mathrm{RMSE}^{(\mathrm{a})}$ & 0.0144 & 0.0069 & 0.0075 & 0.0082 & 0.0050 \\
$\mathrm{R}^{2(\mathrm{~b})}$ & 0.9827 & 0.9943 & 0.9948 & 0.9941 & 0.9908 \\
\hline
\end{tabular}

(a) Root-mean squared error at optimum $\left(t=t_{2}\right)$.

(b) Coefficient of variation at optimum $\left(t=t_{2}\right)$.

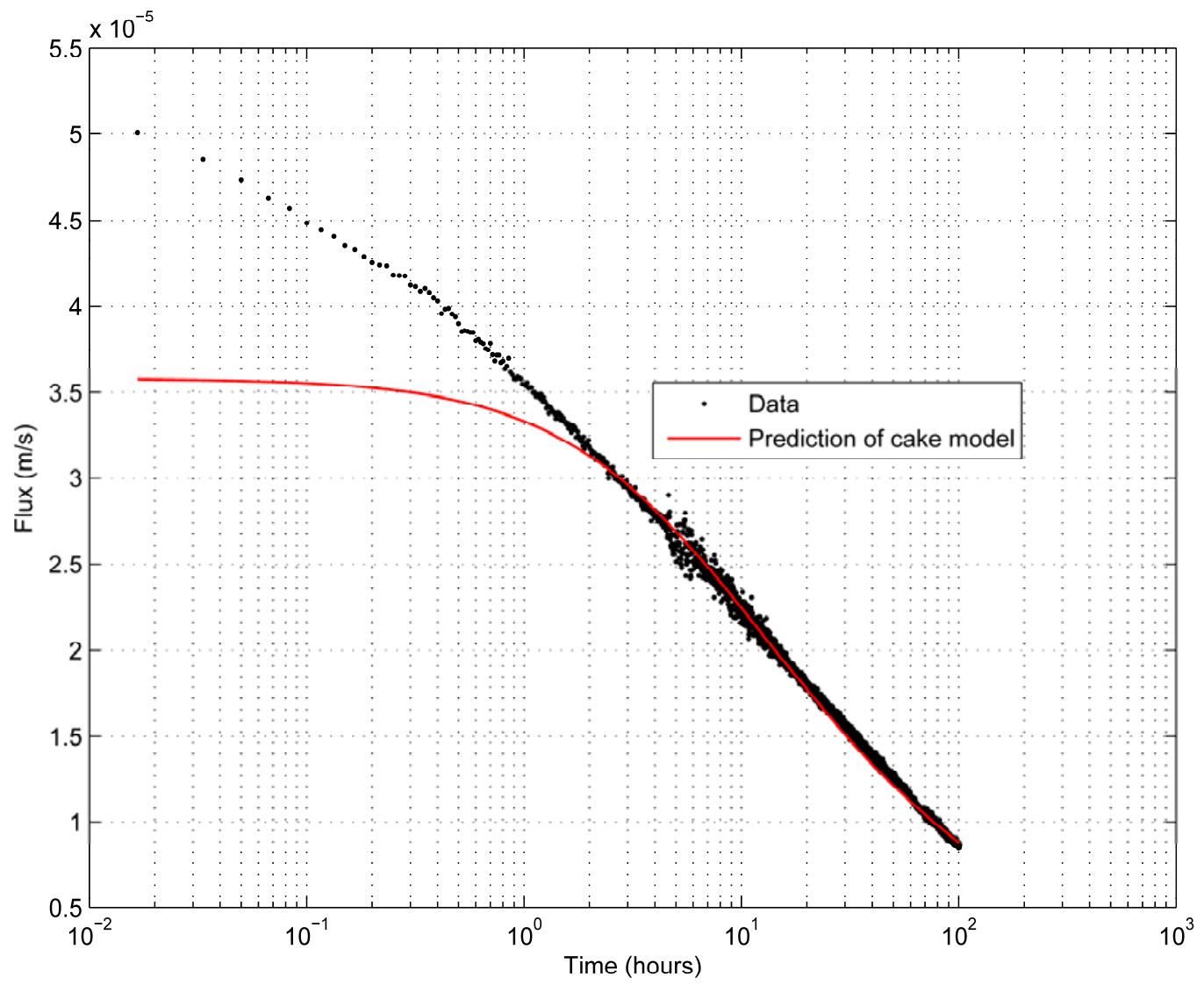

Figure 8.16. Intermediate Time Optimum Fit for S1T1 Data Using the Cake Mechanism Model 


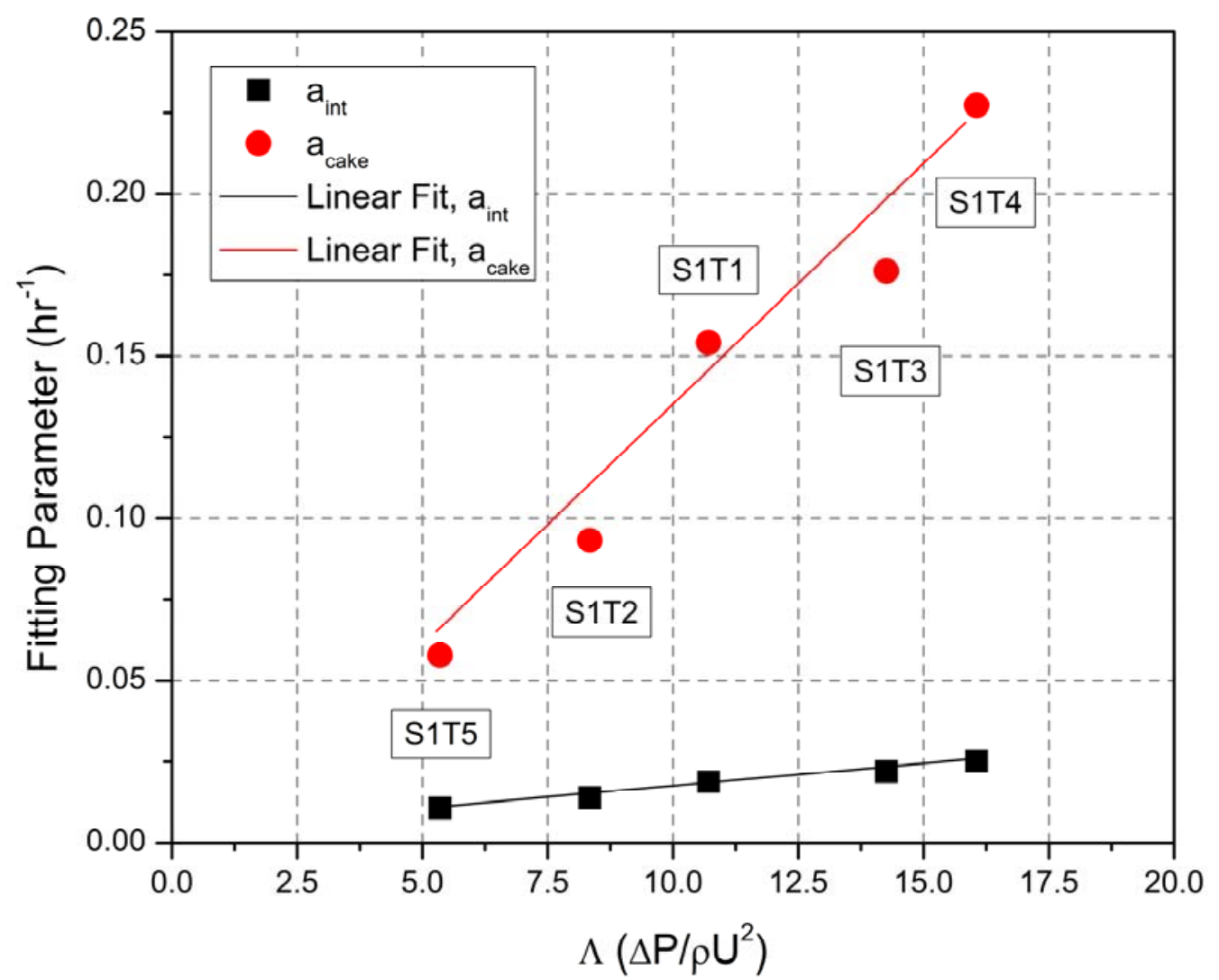

Figure 8.17. Fitting Parameters Obtained for the Optimal Intermediate (long-times) Mechanism and the Cake (intermediate times) Mechanisms vs. the Ratio of the Transmembrane Pressure to the Inertial Pressure. A typical linear fit is shown for reference.

Other models developed in the literature for other applications were also applied to the Series 1 data. Two examples are the combined model of Peng and Tremblay (2008) and the combined models of Bolton et al. (2006). These are models that also use mechanistic formulations to predict the flux with time. They are very similar to the modeling efforts described in this section, in that they have a couple adjustable parameters (the Bolton et al. (2006) combined models have two and the Peng and Tremblay (2008) combined model has three) and they struggle to accurately model the entire 100 hour period.

\subsection{Implication to Operations Strategy}

The Series 1 flux data and the accompanying mechanistic models indicates that the filter will continue to experience flux degradation as elapsed time increases (greater than 100 hours), effectively behaving as a dead-end filtration system. This is an important observation for long-term filtration operation. If the primary mechanism responsible for degrading flux was a membrane fouling/pore sealing phenomenon, then it would be expected that the cake, once formed, would protect the membrane (at least in part). Instead, it was observed that the cake contributes to further flux decline, and that decline is later controlled by the emergence of a second phenomenon, the intermediate mechanism.

The model, then, has two major roles in filter operation strategy. First, it can be used to predict flux at long-times and estimate performance within the range of $\Lambda$ tested so far. These predictions suggest that 
the flux will decay to unacceptable fluxes if no operational actions are taken by approximately 100 hours of elapsed time. Similar fluxes are expected at full scale (see Daniel et al. (2009b), and thus, this is a concern for WTP operations.

Second, the nature of the flux decline suggests that using backpulses is a good way to improve average filter flux. If the flux after around 5 hours of elapsed time decays by cake formation, whether surface or sub-surface cake, then it is beneficial to disrupt the settled particles periodically. Though this will expose the pores to particles more often, cake maturation and intermediate blocking mechanisms are a more serious problem over long operation times. With some additional data, a backpulse strategy could be created to minimize fouling over time; using the model in conjunction with new data could even provide an optimum backpulse frequency.

\subsection{Limitations to Model and Required Testing Needed}

The successful mechanistic modeling of Series 1 flux data is subject to some limitations that are worth discussing in additional detail. The modeling effort was concerned with one complex waste simulant. This simulant was tested at five combinations of TMP and AV for 100 hours. At this point in the model development, it should only be used to predict flux behavior in operational regimes bounded by the conditions tested: large extrapolations are not recommended. The model also does not describe how changes in physical properties, such as density, viscosity, temperature, or solids concentration, affect the flux dynamics. Note also that the original PNNL model fit 12-hour flux data well but was not predictive when extrapolated to longer times. The same caution should be exercised when extrapolating the current mechanistic models past 100 hours.

The mechanism(s) that describe the filter flux at short-times have not yet been determined. An approach identical to the one used to investigate the four blocking laws at long and intermediate times was applied to short-times (approximately 5 hours or less); however, none of the blocking laws were a good fit to the data. Modeling short-time flux dynamics is complicated by the inherent variability in initial filtration behavior; this variability is most likely due to cleaning effectiveness and start-up conditions. Short-time modeling is also more dependent than longer time models on accurate estimation of the initial flux $J_{o}$, since all data is normalized by this value.

Some success in modeling short-time dynamics was achieved using a model given by the form

$$
\frac{J}{J_{o}}=c_{1}-c_{2} \ln \left(t+c_{3}\right)
$$

where $c_{1}, c_{2}$, and $c_{3}$ are all adjustable constants. The reason for the efficacy of Equation 8.27 is not yet known; a model that gives some physical insight into the behavior is highly preferred.

Despite the excellent fit of the Series 1 data with the cake and intermediate mechanism models, the transition between the two segments is not well described. ${ }^{9}$ The model, in its current form, is piecewise

9 The fit is described as excellent because the residuals are normally distributed with a mean of zero at the $95 \%$ confidence level, the root-mean squared error of the fit is always less than $1 \%$ of the full range of values, and for one measure of goodness-of-fit ( $\chi^{2}$ test), the model confirms that the residuals are randomly distributed about zero at the $95 \%$ confidence level. 
and switches abruptly from one mechanism to the other. There may be a gradual change from one to the other than can only be captured by a model that combines both mechanisms. This is desirable both to reduce the amount of information needed to predict flux, but also to make the model continuous to permit mathematical operations such as integration or differentiation. Ideally, a future short-time model could be combined with a revised cake-intermediate model to give a robust model over the entire filtration time.

The mechanistic model also does not preserve the reversible/irreversible distinction found in the original model of Equations 8.1 through 8.4. This is a limitation because it does not lend itself readily to predicting what happens when the filter is backpulsed. In order to understand backpulsing and its effect, the short-time dynamics need to be better understood. Understanding this has important implications for operational strategy, as mentioned in Section 8.3.

Given these limitations, several experimental investigations can be conceived that will improve the model and understanding of the range of filtration behavior. In no particular order, the suggested future investigations are the following:

1. Filtration testing for a period of greater than 100 hours: the conclusion that the cross-flow filter is performing as a dead-end filtration system is important. However, due to flowmeter accuracy limits, the test could not be continued for longer than 100 hours. This is worth pursuing to verify the transition between mechanisms is real and not an artifact of a slow approach to an as-yet unknown steady flux.

2. Parametric testing to assess effects of other operational variables: this will help improve predictions for filtration performed outside the current limits of applicability.

3. Shorter-duration tests: used to assess shorter time flux dynamics and help determine how to use backpulses to maximize filter performance.

4. Tests at other solids concentrations: different solids concentrations will affect particle-particle and particle-surface interactions; testing them will help verify the range of applicability of the model.

5. Tests with other waste simulants: validate the model approach against slurries with different constituents than the one used in Series 1 testing. This could also support more a powerful predictive ability than currently exists in the model.

These types of tests will support expanding the model to describe flux behavior in this type of cross-flow system for a wider range of parameters and conditions. They will also aid in the development of an optimum backpulse strategy for long-time operations. 


\subsection{Summary and Recommendations}

The goal of the current report was to provide a summary update of the testing activities for the WP-2.3.6 task at PNNL under the EM31 program and provide a status of our model. Several test activities were undertaken to improve understanding of the filtration behavior of high-level wastes and waste simulant. These tests, conducted with a high-level tank waste simulant and simulant components, included:

- Series 1 Testing: Long-time filtration and filter fouling tests, as described in Section 3.1 (approach) and Section 5 (results), were conducted to better understand flux decline over testing periods longer than previously explored in high-level waste filtration studies performed at PNNL.

- Series 2 Testing: Individual simulant component tests, as described in Section 3.2 (approach) and Section 6 (results), were conducted to identify which simulant components contributed to the overall flux behavior of the high-level waste simulant and to better understand how solution chemistry impacted filtration.

- Series 3 Testing: Cleaning tests, as described in Section 3.3 (approach) and Section 7 (results), were conducted to determine the efficacy of rinsing and acid cleaning (with solutions of nitric and oxalic acid) in restoring filter flux and to evaluate a clean water flux metric by which cleaning effectiveness may be identified.

These tests, and in particular Series 1 testing, provide data needed to validate and improve existing PNNL models used to describe and predict the filtration behavior of high-level wastes. Modeling improvement efforts, as described in Section 3.4 (approach) and Section 8 (results), conducted as part of the current study have allowed extension of the model to times comparable to those expected for high-level waste filtration operations at high-level waste treatment facilities like WTP and also incorporate predictive capabilities with respect to process operating conditions such as filter flow velocity (i.e., axial velocity) and filter transmembrane pressure. The latter improvements to the model provide a necessary tool for optimizing high-level waste filtration operations with respect to process operating conditions.

While the FY10 testing program answered many questions about filtration performance, the following testing is planned in FY11 to resolve:

- 400-Hour Testing. The findings outlined above have significant impact on filtration operations at WTP. First, the lack of observed filtration steady-state after 100 hours has significant implications on the throughput of filtration operations greater than 100 hours. Indeed, the current tests suggest that the filter will dead-end (go to zero flux) at finite time, necessitating flux recovery operations such as backpulsing or chemical cleaning. Extrapolation of filter flux results for one set of operating conditions indicates that the zero-flux time is $\sim 400$ hours. As such, PNNL recommends a 400 -hour filter test to verify the observed long-time flux behavior.

Backpulse Recovery Testing. The long-time test results indicate a strong need for flux recovery operations. Backpulsing was shown to effectively restore a significant portion of the filter flux. However, a portion of the flux ( $25 \%$ ) could not be recovered by the single set of backpulses applied after 100 hours of continuous filtration. Irreversible flux loss with respect to backpulse is cause for concern, as the underlying mechanisms are not well understood. Additional testing is needed to 
confirm if irreversible fouling (filter conditioning) has a steady-state value or will continue to degrade flux recovered upon backpulsing.

- TMP/AV Cake Resistance Testing. Finally, the long-time AV and TMP results are significant in that they provide insight into how the filtration process can be optimized to improve filter throughput. The throughput results for the first 100 hours of testing are shown in Table 5.5. The results are surprising, as they show that for 100 hours of undisturbed filtration, throughput can be improved by increasing axial velocity and lowering transmembrane pressure. This contrasts with previous short-time test results, which said increased axial velocity and transmembrane pressure were needed to improve throughput. As such, the current tests illustrate the importance of understanding fouling dynamics with respect to the selected operating conditions as non-obvious process optimizations (such as lower transmembrane pressure) may result in better filter performance.

- Temperature/Concentration Testing. All filtration tests conducted with the high-level waste simulant in the current study employed a single slurry test concentration and temperature of $\sim 5$-wt $\%$ and $25^{\circ} \mathrm{C}$, respectively. As such, it is unknown if the current improvements to the PNNL filtration model properly incorporate slurry concentration and temperature functionality. Filtration tests where slurry concentration and filtration temperature are varied over ranges expected in typical high-level waste operations are needed to allow further model development and process optimization.

- Direct Imaging of the Filter Element. Testing has identified irreversible fouling of the filter element with respect to backpulsing that appears to necessitate chemical cleaning operations. To date, filtration and cleaning tests with both high-level waste simulant and individual simulant components have not clearly identified the particle species and/or chemistry that yield the observed irreversible fouling. Direct imaging of the filter surface with optical microscopy or SEM, along with compositional analysis of particulates observed on the filter surface, is recommended to help identify species that irreversibly foul the filter elements. Successful identification of particle chemistries that foul strongly improves predictive capabilities with respect to which actual high-level waste chemistries may challenge high-level waste filtration throughput.

- Development of New Simulants. The high-level waste simulant developed for Integrated Engineering-Scale Testing at PNNL's Pretreatment Engineering Platform has been used extensively to improve understanding of high-level waste filtration. However, this simulant is not necessarily representative of the broad range of high-level chemistries that may be encountered in practice. Development of new simulants, such as a saltcake simulant that represents waste types such as bismuth phosphate saltcakes, S-saltcakes, etc. for use in filtration studies, is needed to evaluate the overall efficacy of filtration in high-level waste separation processes. 


\subsection{References}

Benavente J and C Fernandez-Pineda. 1985. "Electrokinetic Phenomena in porous membranes: Determination of phenomenological coefficients and transport numbers." Journal of Membrane Science 23:121-136.

Billing JM, RC Daniel, DE Kurath, and RA Peterson. 2009. Bench-Scale Filtration Testing in Support of the Pretreatment Engineering Platform (PEP). PNNL-18673, WTP-RPT-203, Pacific Northwest National Laboratory, Richland, Washington.

Bolton G, D LaCasse, and R Kuriyel. 2006. "Combined models of membrane fouling: Development and application to microfiltration and ultrafiltration of biological fluids." Journal of Membrane Science 277:75-84.

Broz Z and N Epstein. 1976. "Electrokinetic flow through porous media composed of fine cylindrical capillaries." Journal of Colloid and Interface Science 56(3):605-612.

Celis, M, JE Dennis, and RA Tapia. 1985. "A trust region strategy for nonlinear equality constrained optimization.” In Numerical Optimization 1984 (P. Boggs, R. Byrd and R. Schnabel, eds), Philadelphia: SIAM, pp. 71-82.

Daniel RC, PP Schonewill, RA Peterson, and RW Shimskey. 2010a. A Brief Review of Filtration Studies for Waste Treatment at the Hanford Site. PNNL-20023, Pacific Northwest National Laboratory, Richland, Washington.

Daniel RC, JM Billing, RL Russell, RW Shimskey, HD Smith, and RA Peterson. 2010b. "Integrated pore blockage-cake filtration model for crossflow filtration." Accepted for publication in CHEMICAL ENGINEERING RESEARCH AND DESIGN.

Daniel RC, JM Billing, KJ Cantrell, ML Luna, RA Peterson, ML Bonebrake, RW Shimskey, and LK Jagoda. 2009a. Characterization of Filtration Scale-Up Performance. PNNL-18117, WTP-RPT-168, Rev. 0, Pacific Northwest National Laboratory, Richland, Washington.

Daniel RC, JM Billing, JR Bontha, CF Brown, PW Eslinger, BD Hanson, JL Huckaby, NK Karri, ML Kimura, DE Kurath, and MJ Minette. 2009b. EFRT M-12 Issue Resolution: Comparison of Filter Performance at PEP and CUF Scale. PNNL-18498, Pacific Northwest National Laboratory, Richland, Washington.

Edwards MK, JM Billing, DL Blanchard, Jr, EC Buck, AJ Casella, AM Casella, JV Crum, RC Daniel, KE Draper, SK Fiskum, LK Jagoda, ED Jenson, AE Kozelisky, PJ MacFarlan, RA Peterson, RW Shimskey, LA Snow, and RG Swoboda. 2009. Characterization, Leaching, and Filtration Testing for Tributyl Phosphate (TBP, Group 7) Actual Waste Sample Composites. PNNL-18119, Pacific Northwest National Laboratory, Richland, Washington. 
Fiskum SK, JM Billing, JV Crum, RC Daniel, MK Edwards, RW Shimskey, RA Peterson, PJ MacFarlan, EC Buck, KE Draper, and AE Kozelisky. 2009. Characterization, Leaching, and Filtrations Testing of Ferrocyanide Tank sludge (Group 8) Actual Waste Composite. PNNL-18120, Pacific Northwest National Laboratory, Richland, Washington.

Geeting JGH, RT Hallen, LK Jagoda, AP Poloski, RD Scheele, DR Weier. 2003. Filtration, Washing, and Caustic Leaching of Hanford Tank AZ-101 Sludge. PNWD-3206, Rev 1, WTP-RPT-043, Rev. 1, Battelle, Pacific Northwest Division, Richland, Washington.

Geeting JGH, RL Aaberg, OP Bredt, CA Burns, EC Golovich, CE Guzman-Leong, GB Josephson, DE Kurath, and GJ Sevigny. 2009. Pretreatment Engineering Platform (PEP) Integrated Test B Run Report-Caustic and Oxidative Leaching in UFP-VSL-T02A. PNNL-18723, Pacific Northwest National Laboratory, Richland, Washington.

Guzman-Leong CE, OP Bredt, CA Burns, RC Daniel, JGH Geeting, EC Golovich, GB Josephson, DE Kurath, GJ Sevigny, DM Smith, YF Su, PLJ Valdez, ST Yokuda, and JK Young. 2009. PEP Run Report for Integrated Test A; Caustic Leaching in UFP-VSL-T01A, Oxidative Leaching in UFP-VSL-T02A. PNNL-18755, Pacific Northwest National Laboratory, Richland, Washington.

Hermia J. 1981. "Constant Pressure Blocking Filtration Laws - Application to Power-Law Non-Newtonian Fluids.” Transactions of the Institution of Chemicals Engineers 59(1):183-187.

Huisman IH, G Tragardh, C Tragardh, and A Pihlajamaki. 1998. "Determining the zeta-potential of ceramic microfiltration membranes using the electroviscous effect." Journal of Membrane Science 147:187-194.

Johnson CE and MR Duignan. 2011. Crossflow Filtration: Literature Review. SRNL-STI-2011-00013. Savannah River National Laboratory, Aiken, South Carolina.

Kurath DE, BD Hanson, MJ Minette, DL Baldwin, BM Rapko, LA Mahoney, PP Schonewill, RC Daniel, PW Eslinger, JL Huckaby, JM Billing, PS Sundar, GB Josephson, JJ Toth, ST Yokuda, EBK Baer, SM Barnes, EC Golovich, SD Rassat, CF Brown, JGH Geeting, GJ Sevigny, AJ Casella, JR Bontha, RL Aaberg, PM Aker, CE Guzman-Leong, ML Kimura, SK Sundaram, RP Pires, BE Wells, and OP Bredt. 2009. Pretreatment Engineering Platform Phase 1 Final Test Report. PNNL-18894; WTP-RPT-197, Rev 0, Pacific Northwest National Laboratory, Richland, Washington.

Lawrence ND, JM Perera, M Iyer, MW Hickey, and GW Stevens. 2006. "The use of streaming potential measurements to study the fouling and cleaning of ultrafiltration membranes." Separation and Purification Technology 48:106-112.

Levenberg K. 1944. "A Method for the Solution of Certain Non-Linear Problems in Least Squares." The Quarterly of Applied Mathematics 2: 164-168.

Lumetta GJ, EC Buck, RC Daniel, K Draper, MK Edwards, SK Fiskum, RT Hallen, LK Jagoda, ED Jenson, AE Kozelisky, PJ MacFarlan, RA Peterson, RW Shimskey, SI Sinkov, and LA Snow. 2009. Characterization, Leaching, and Filtration Testing for Bismuth Phosphate Sludge (Group 1) and Bismuth Phosphate Saltcake (Group 2) Actual Waste Sample Composites. PNNL-17992, Pacific Northwest National Laboratory, Richland, Washington. 
Marquardt D. 1963. “An Algorithm for Least-Squares Estimation of Nonlinear Parameters.” SIAM Journal on Applied Mathematics 11: 431-441.

Martinez F, A Martin, J Malfeito, L Palacio, P Pradanos, F Tejerina, and A Hernandez. 2002.

"Streaming potential through and on ultrafiltration membranes: Influence of salt retention." Journal of Membrane Science 206:431-441.

McCarthy AA, PK Walsh, and G Foley. 2002. "Characterising the packing and dead-end filter cake compressibility of the polymorphic yeast Kluyveromyces marxianus var. marxianus NRRLy2415." Journal of Membrane Science 198:87-94.

Nash, CA, SW Rosencrance, and WR Wilmarth. 2000. Strontium-Transuranic Precipitation and Crossflow Filtration of 241-AN-102 Large C. BNF-033-98-0317, Savannah River Technology Center, Aiken, South Carolina.

Nystrom M, M Lindstrom, and E Matthiasson. 1989. "Streaming Potential as a Tool in the Characterization of Ultrafiltration Membranes." Colloids and Surfaces 36:297-312.

Peng H and AY Tremblay. 2008. "Membrane regeneration and filtration modeling in treating oily wastewaters." Journal of Membrane Science 324:59-66.

Poirier MR and SD Fink. 2002. Investigation of Alternative Approaches for Cleaning Mott Porous Metal Filters. WSRC-TR-2002-00526, Rev. 0, Savannah River National Laboratory, Savannah River Site, South Carolina.

Rorabacher DB. 1991. "Statistical Treatment for Rejection of Deviant Values: Critical Values of Dixon Q Parameter and Related Subrange Ratios at the 95 percent Confidence Level." Analytical Chemistry 63(2):139-146.

Rubow KL and S Jha. 1999. "Sintered Metal Microfiltration Media." Presented at the Seventeenth Annual Membrane Technology/Separations Planning Conference, Newton, Massachusetts.

Russell RL, DE Rinehart, JM Billing, HD Smith, and RA Peterson. 2009. Development and Demonstration of Ultrafiltration Simulants. PNNL-18090, WTP-RPT-183, Rev 0, Pacific Northwest National Laboratory, Richland, Washington.

Scheele RD, GN Brown, and DE Kurath. 2009. Scale-Up, Production, and Procurement of PEP Simulants. PNNL-18678, Pacific Northwest National Laboratory, Richland, Washington.

Shimskey RW, JM Billing, EC Buck, RC Daniel, KE Draper, MK Edwards, JGH Geeting, RT Hallen, ED Jenson, AE Kozelisky, PJ MacFarlan, RA Peterson, LA Snow, and RG Swoboda. 2009a. Filtration and Leach Testing for REDOX Sludge and S-Saltcake Actual Waste Sample Composites. PNNL-17965, Pacific Northwest National Laboratory, Richland, Washington.

Shimskey RW, RC Daniel, AE Kozelisky, JM Billing, K Draper, PJ MacFarlan, EC Buck, MK Edwards, RA Peterson, AJ Casella, RT Hallen, RG Swoboda, and JV Crum. 2009b. Filtration and Leach Testing for PUREX Cladding Sludge and REDOX Cladding Sludge Actual Waste Sample Composites.

PNNL-18048, Pacific Northwest National Laboratory, Richland, Washington. 
Suzuki O. 2006. “Adsorption and Fouling at Stainless Steel Surfaces." Encyclopedia of Surface and Colloid Science, Volume 1, $2^{\text {nd }}$ Edition, Editor P Somasundaran. 185-192.

Yaws CL. 1999. Chemical Properties Handbook. McGraw-Hill. ISBN 0070734011.

Zeman LJ and AL Zydney. 1996. Microfiltration and ultrafiltration: principles and applications. Marcel Dekker, New York. 
Appendix A 



\section{Appendix A}

This appendix presents figures and tabulated data generated during the modeling exercises described in Section 8 for tests S1T2 through S1T5. The figures and tables are presented without comment; refer to the Section 8 text for more information about each series of plots.

\section{A.1 Original Model}

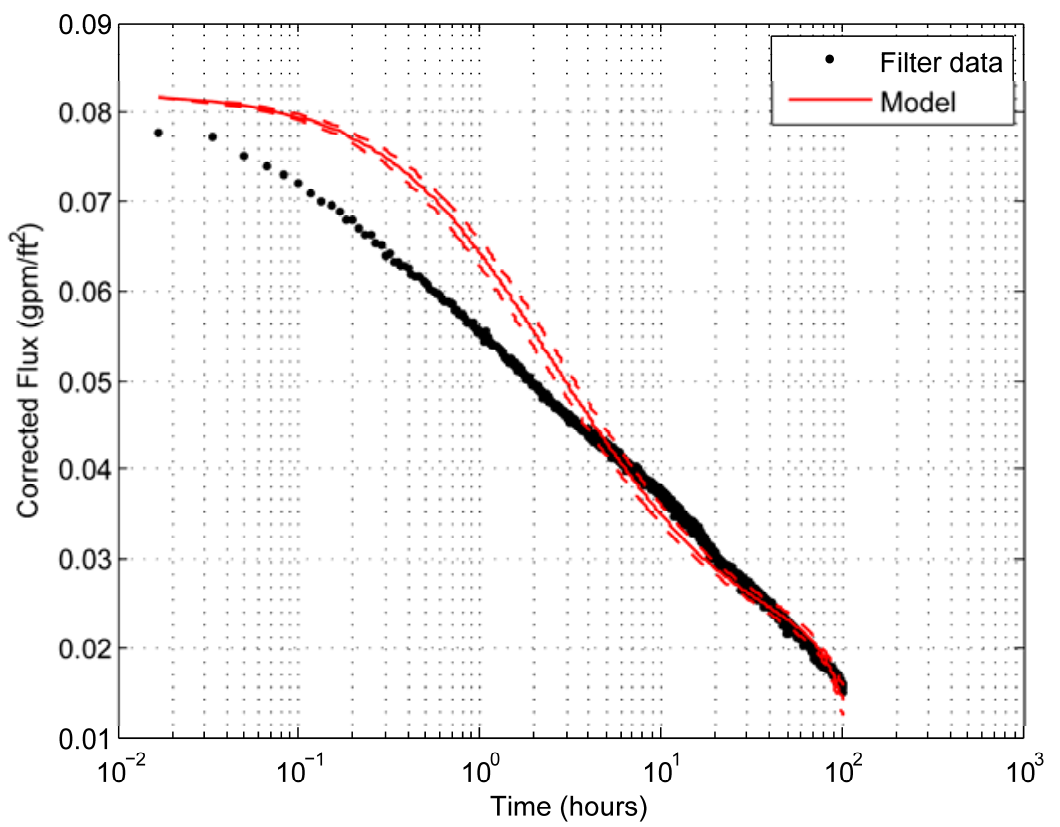

Figure A.1. Original Model Fit to S1T2 Data Before Backpulsing ( $\sim 100$ hours). The dotted lines are the bands of $95 \%$ confidence for the model fit. 


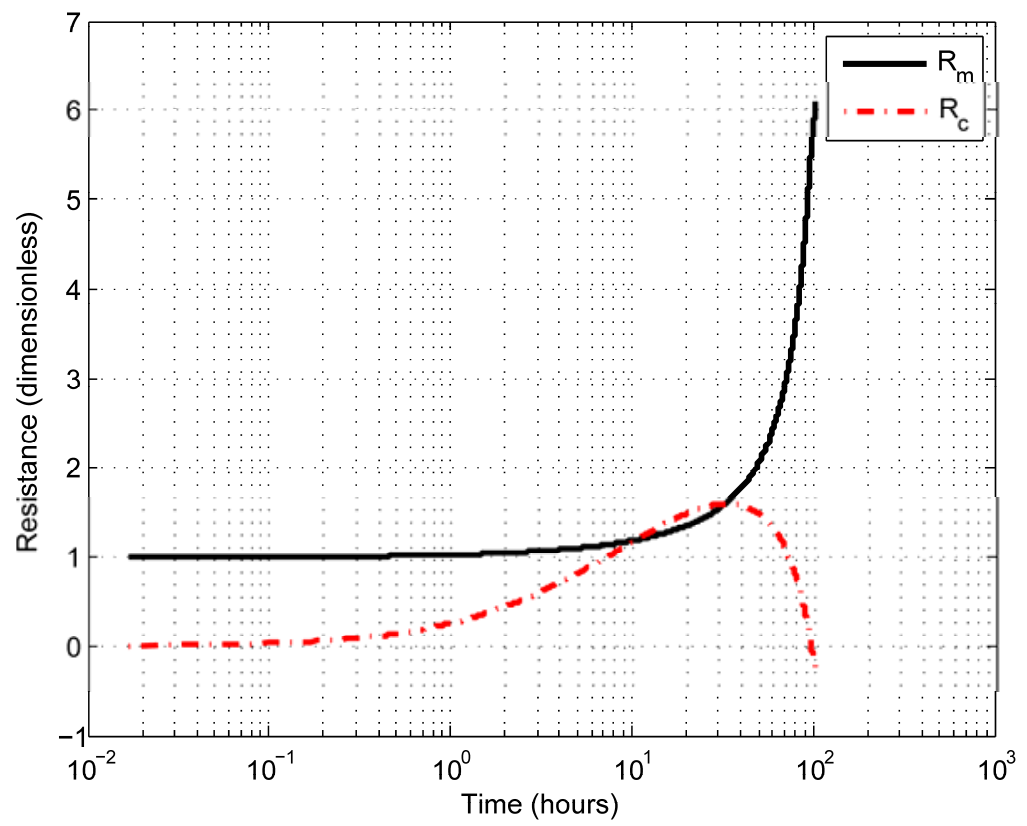

Figure A.2. Dimensionless Membrane $\left(\mathrm{R}_{\mathrm{m}}\right)$ and Cake $\left(\mathrm{R}_{\mathrm{c}}\right)$ Resistance as a Function of Time for the Model Fit to S1T2 Data

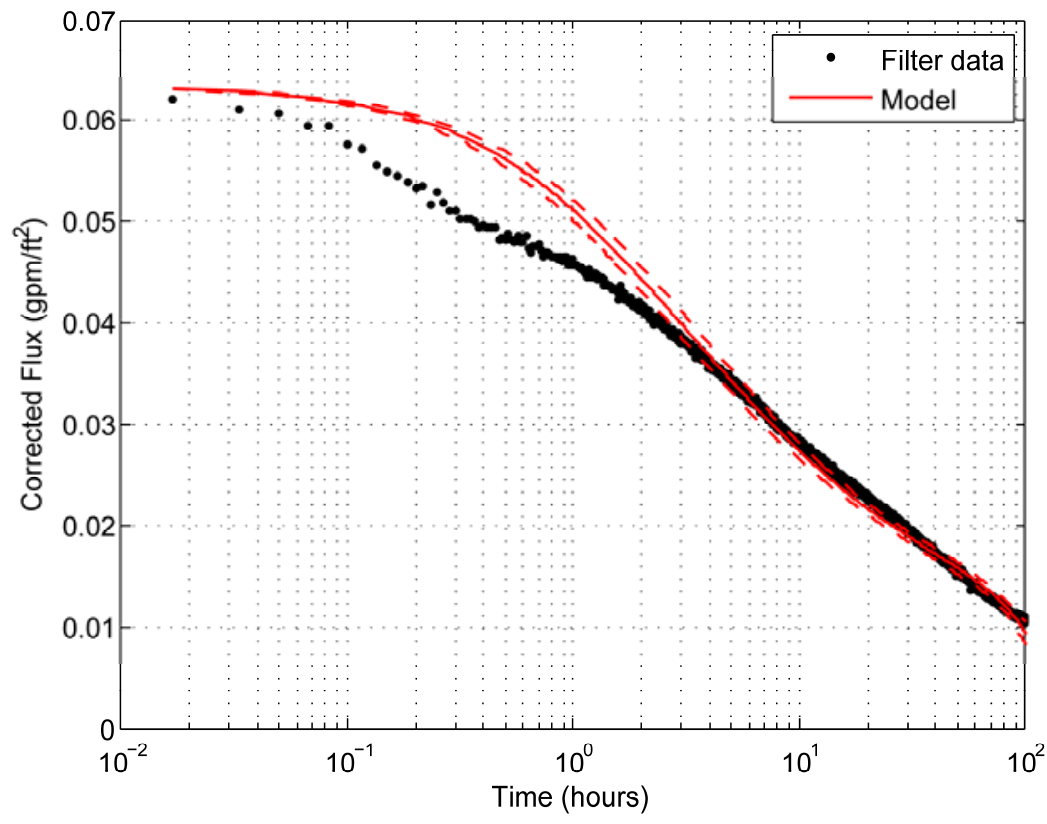

Figure A.3. Original Model Fit to S1T3 Data Before Backpulsing ( 100 hours). The dotted lines are the bands of $95 \%$ confidence for the model fit. 


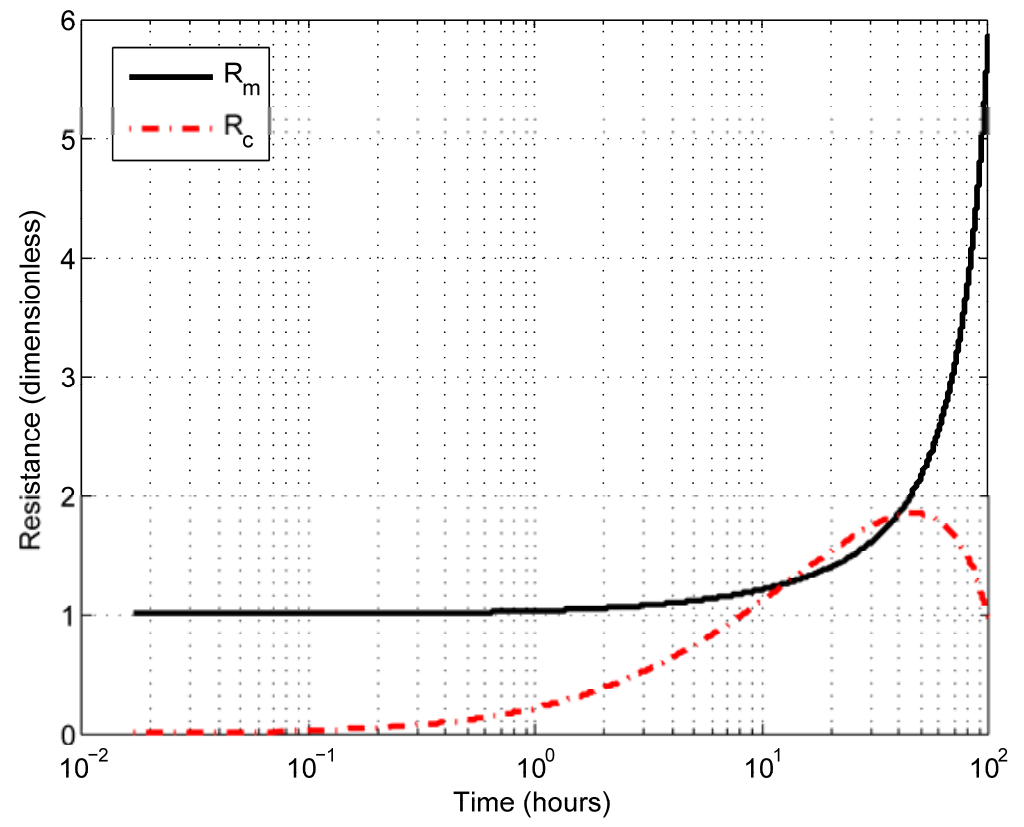

Figure A.4. Dimensionless Membrane $\left(\mathrm{R}_{\mathrm{m}}\right)$ and Cake $\left(\mathrm{R}_{\mathrm{c}}\right)$ Resistance as a Function of Time for the Model Fit to S1T3 Data

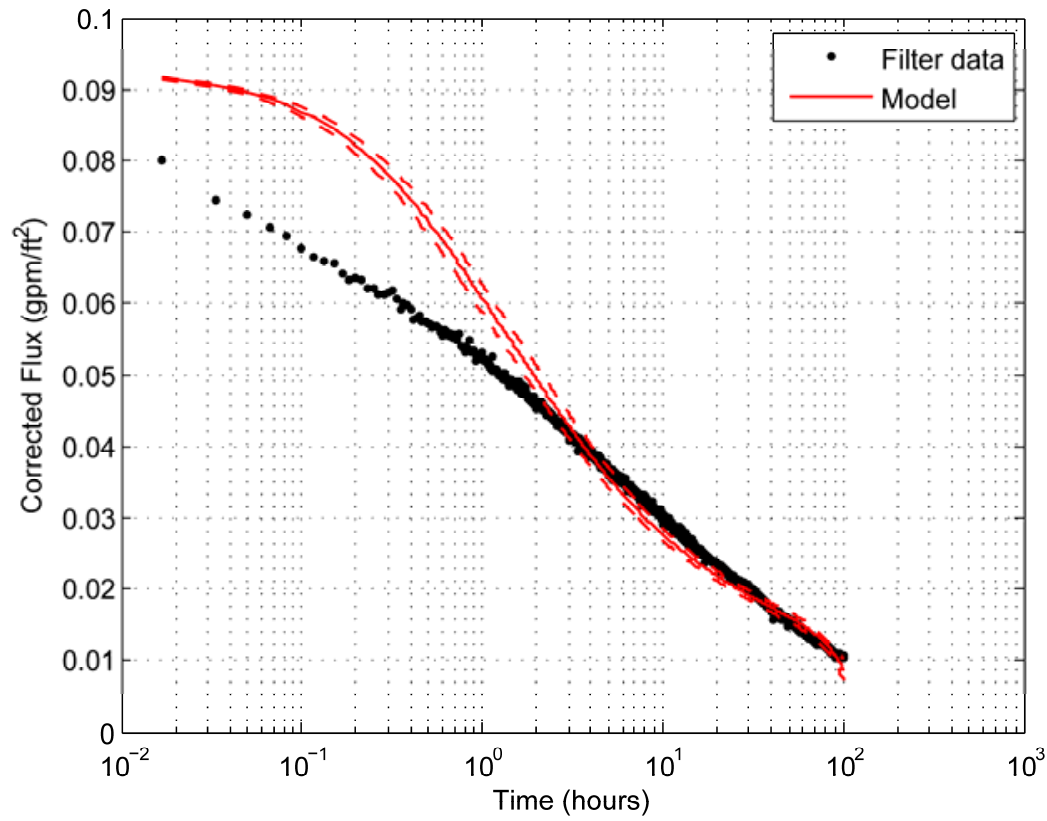

Figure A.5. Original Model Fit to S1T3 Data Before Backpulsing ( $\sim 100$ hours). The dotted lines are the bands of $95 \%$ confidence for the model fit. 


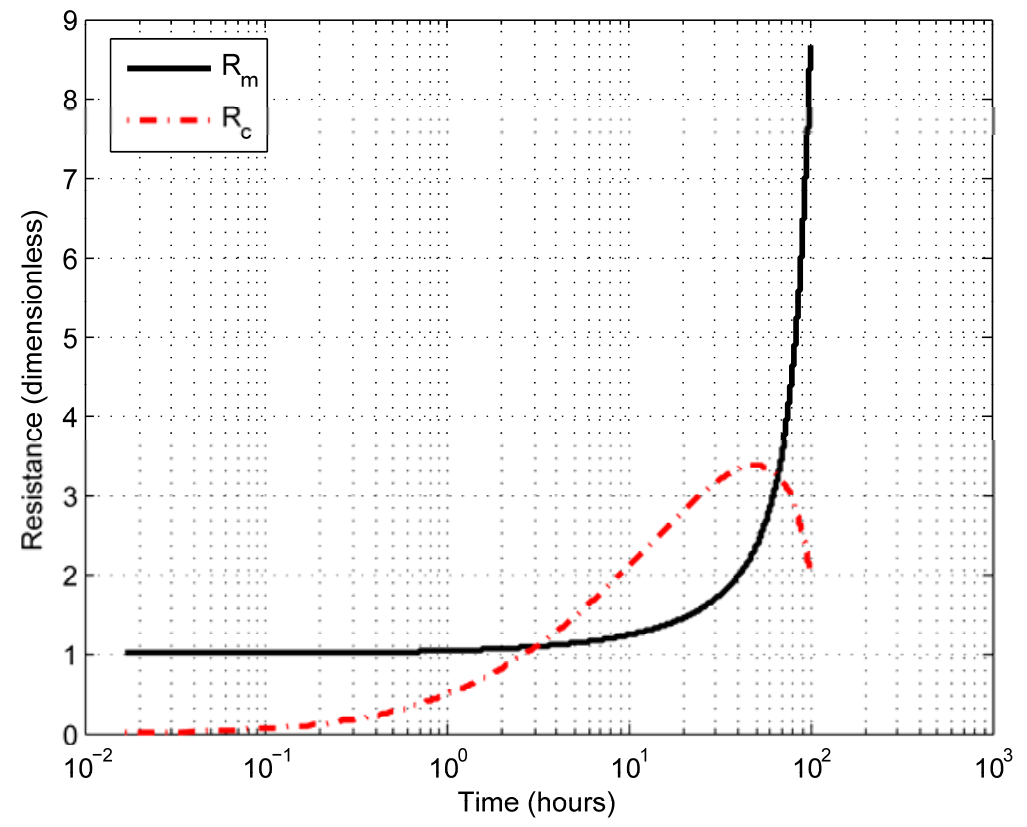

Figure A.6. Dimensionless Membrane $\left(\mathrm{R}_{\mathrm{m}}\right)$ and Cake $\left(\mathrm{R}_{\mathrm{c}}\right)$ Resistance as a Function of Time for the Model Fit to S1T4 Data

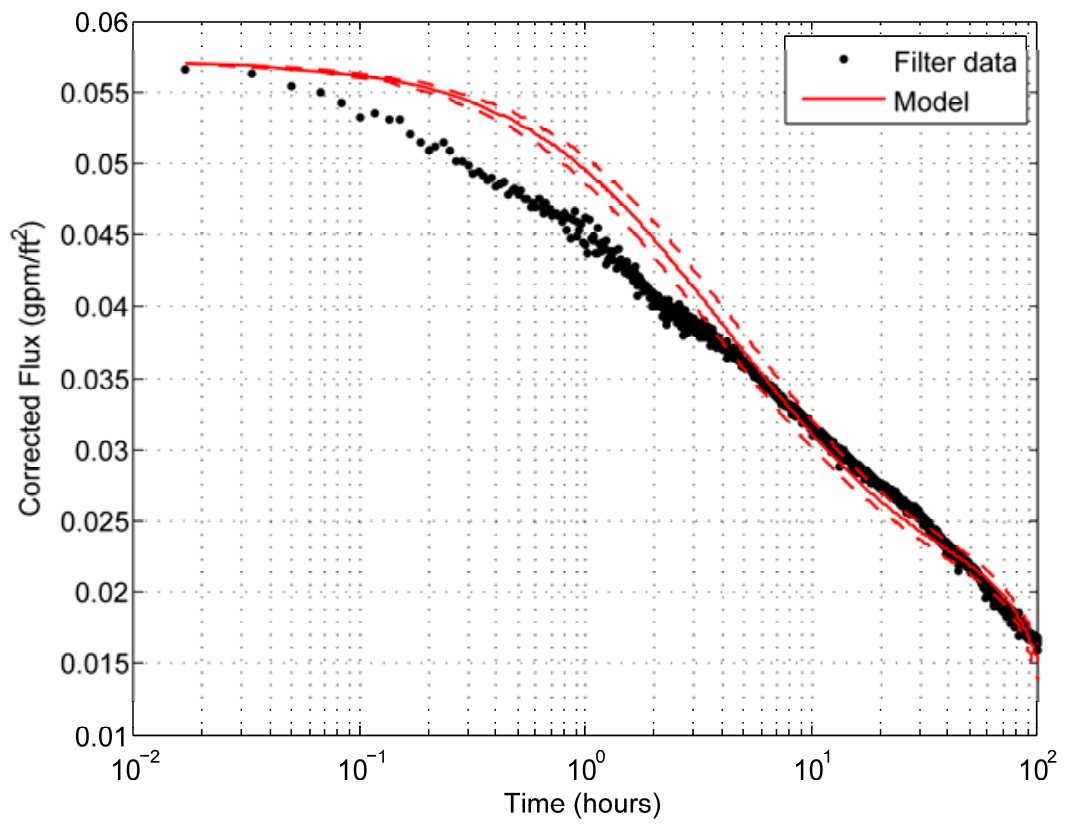

Figure A.7. Original Model Fit to S1T5 Data Before Backpulsing ( $\sim 100$ hours). The dotted lines are the bands of $95 \%$ confidence for the model fit. 


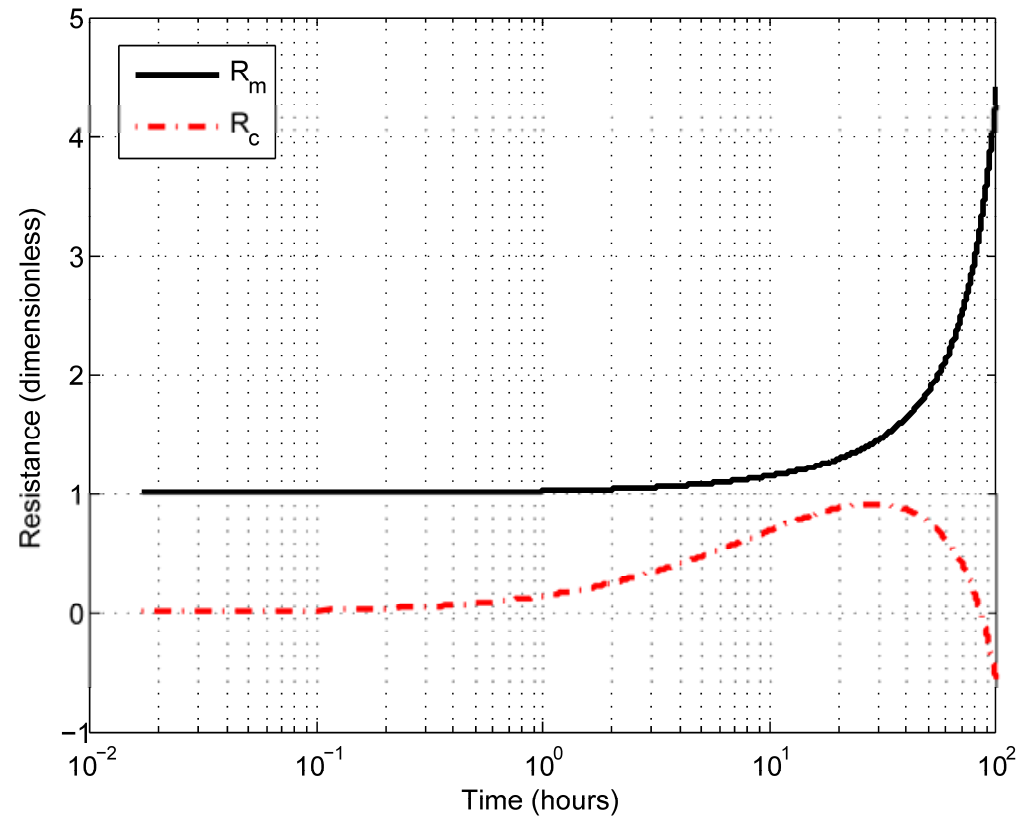

Figure A.8. Dimensionless Membrane $\left(\mathrm{R}_{\mathrm{m}}\right)$ and Cake $\left(\mathrm{R}_{\mathrm{c}}\right)$ Resistance as a Function of Time for the Model Fit to S1T5 Data

\section{A.2 Modified Model with $\mathbf{R}_{\mathrm{m}}{ }^{*}=\mathbf{R}_{\mathrm{c}}{ }^{*}$ Constraint}

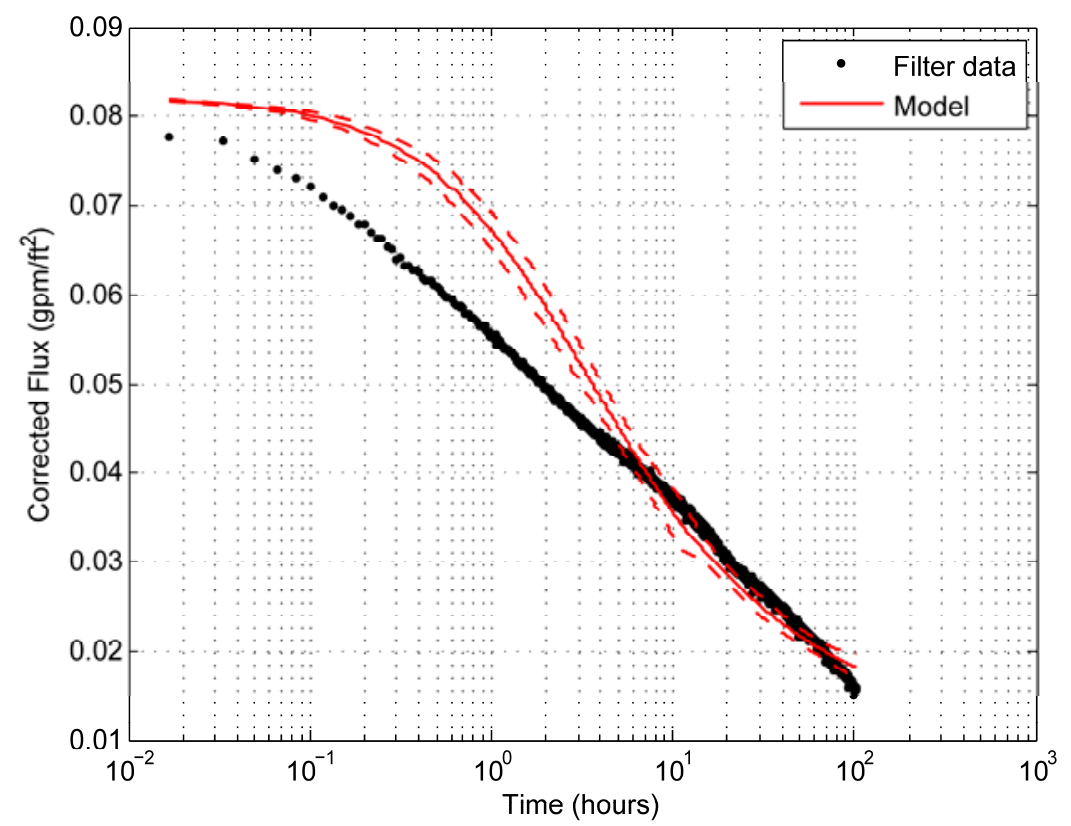

Figure A.9. Modified Model Fit to S1T2 Data Before Backpulsing ( 100 hours) Using Constraint in Equation 8.9. The dotted lines are the $95 \%$ confidence bands for the model fit. 


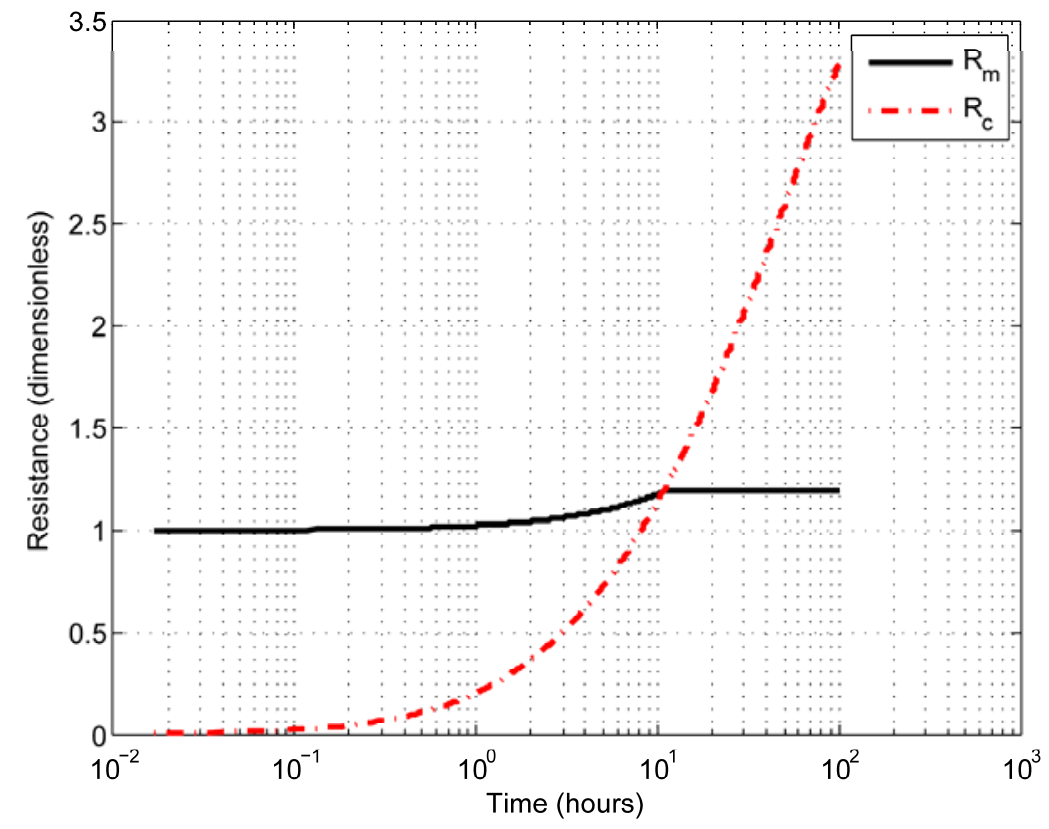

Figure A.10. Dimensionless Membrane $\left(\mathrm{R}_{\mathrm{m}}\right)$ and Cake $\left(\mathrm{R}_{\mathrm{c}}\right)$ Resistance as a Function of Time for the Modified Model Fit to S1T2 Data

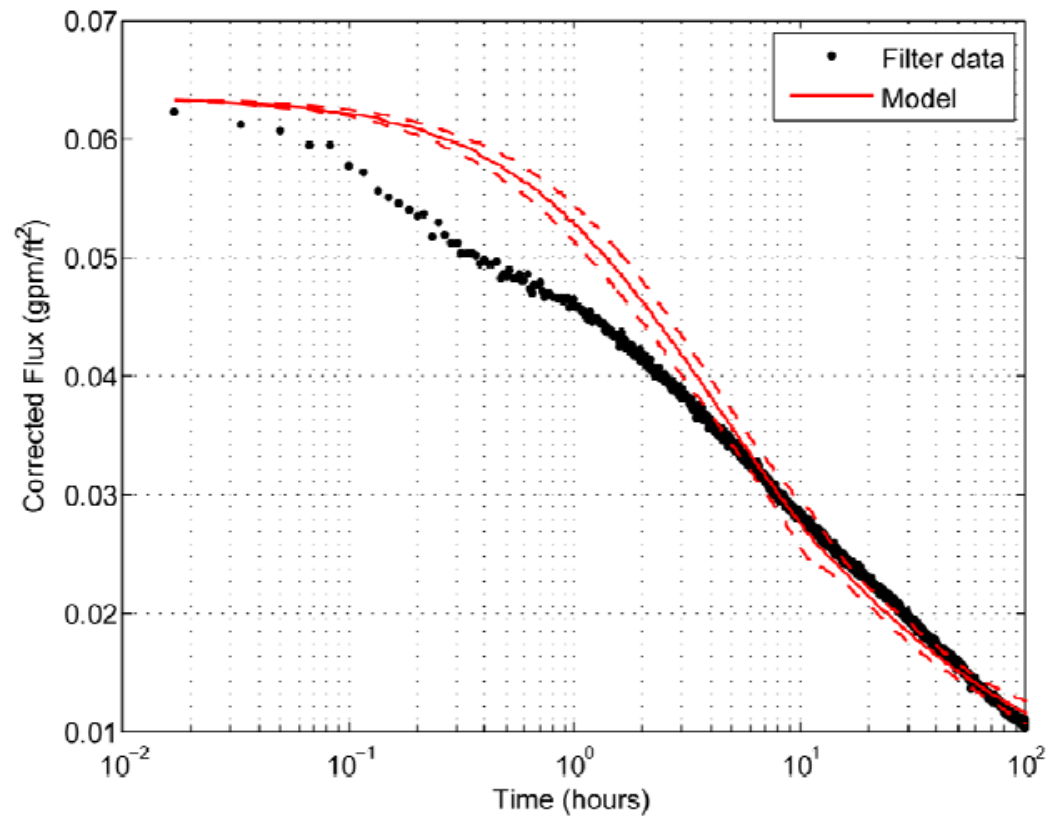

Figure A.11. Modified Model Fit to S1T3 Data Before Backpulsing ( $\sim 100$ hours) Using Constraint in Equation 8.9. The dotted lines are the $95 \%$ confidence bands for the model fit. 


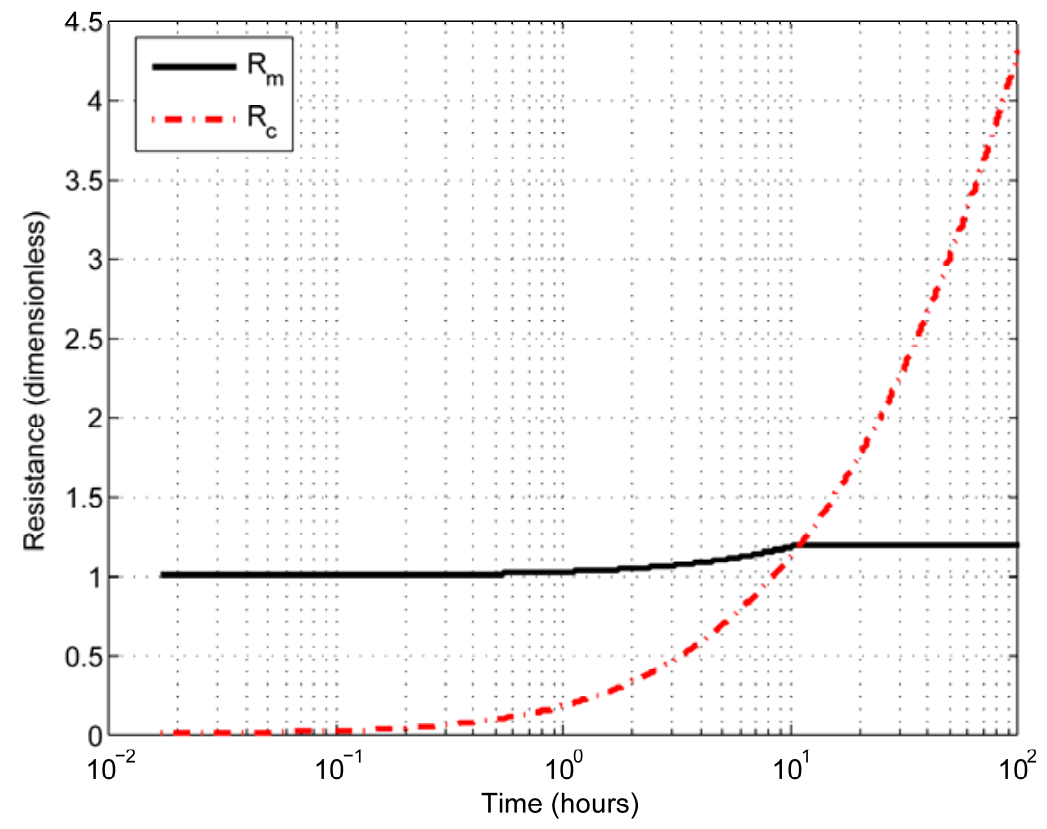

Figure A.12. Dimensionless Membrane $\left(\mathrm{R}_{\mathrm{m}}\right)$ and Cake $\left(\mathrm{R}_{\mathrm{c}}\right)$ Resistance as a Function of Time for the Modified Model Fit to S1T3 Data

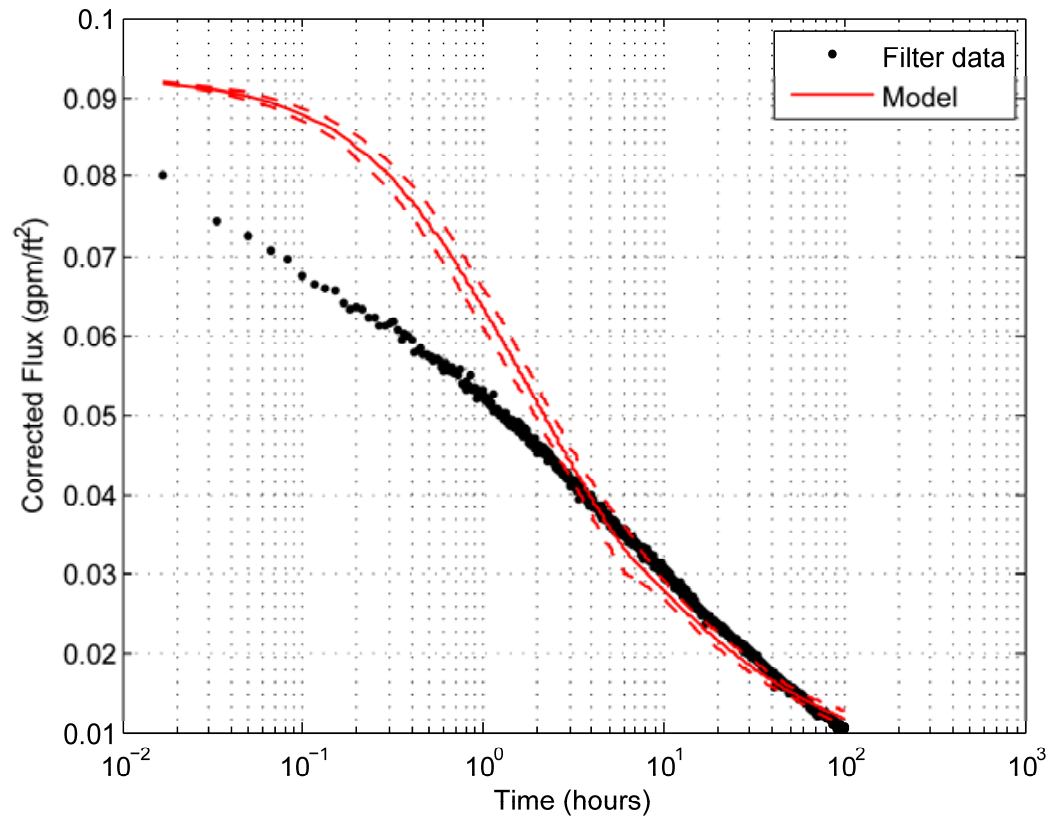

Figure A.13. Modified Model Fit to S1T4 Data Before Backpulsing ( $\sim 100$ hours) Using Constraint in Equation 8.9. The dotted lines are the $95 \%$ confidence bands for the model fit. 


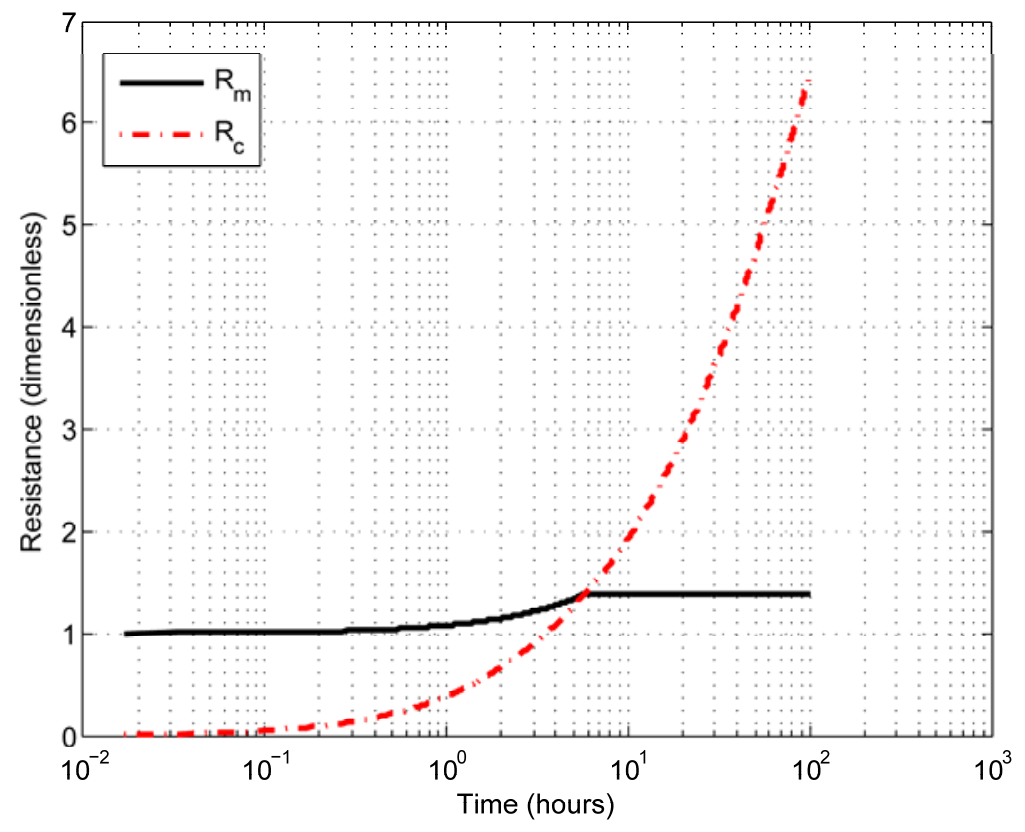

Figure A.14. Dimensionless Membrane $\left(\mathrm{R}_{\mathrm{m}}\right)$ and Cake $\left(\mathrm{R}_{\mathrm{c}}\right)$ Resistance as a Function of Time for the Modified Model Fit to S1T4 Data

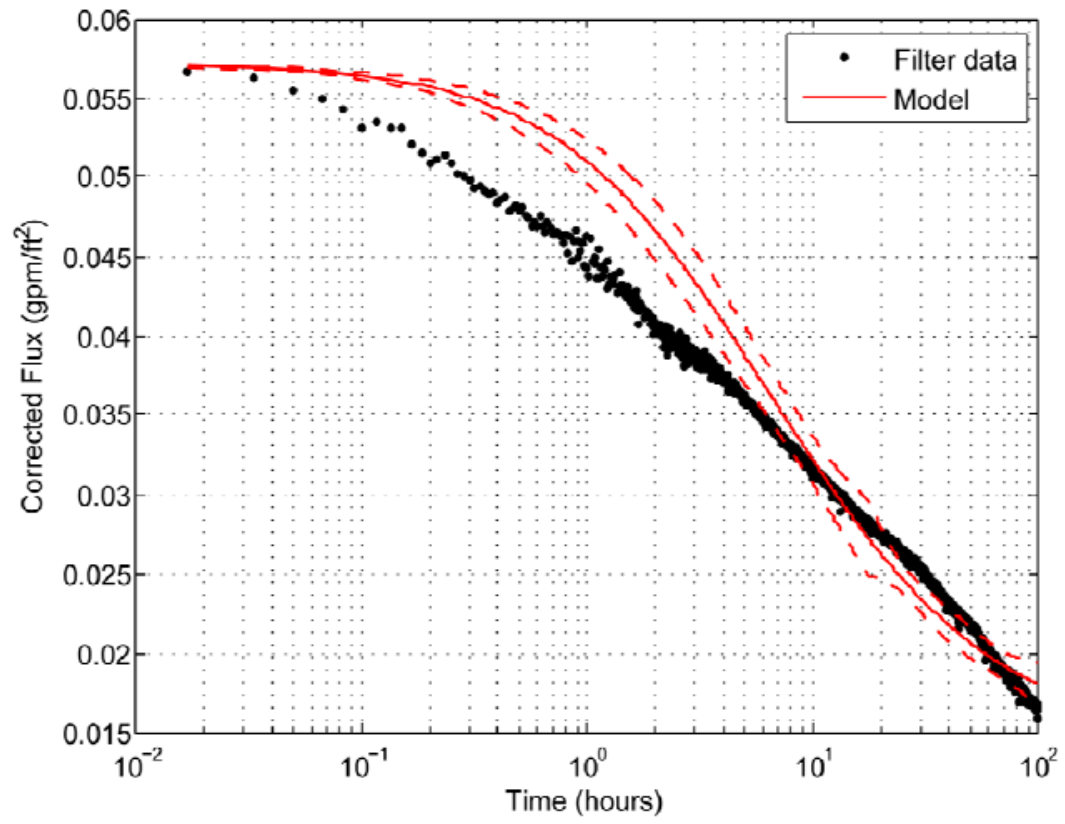

Figure A.15. Modified Model Fit to S1T4 Data Before Backpulsing ( $\sim 100$ hours) Using Constraint in Equation 8.9. The dotted lines are the $95 \%$ confidence bands for the model fit. 


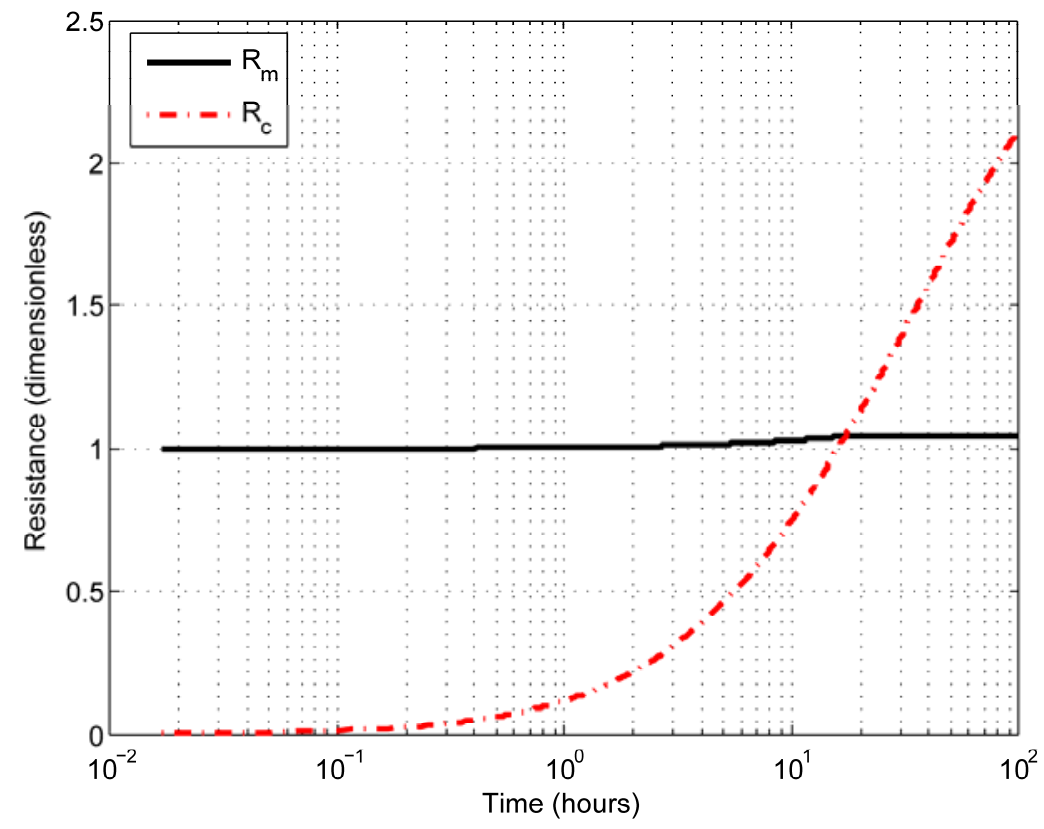

Figure A.16. Dimensionless Membrane $\left(\mathrm{R}_{\mathrm{m}}\right)$ and Cake $\left(\mathrm{R}_{\mathrm{c}}\right)$ Resistance as a Function of Time for the Modified Model Fit to S1T5 Data

\section{A.3 Modified Model with $\mathbf{R}_{\mathrm{m}}{ }^{*}=\mathbf{R}_{\mathrm{c}}{ }^{*}$ Constraint - Including Post-Backpulse Data}

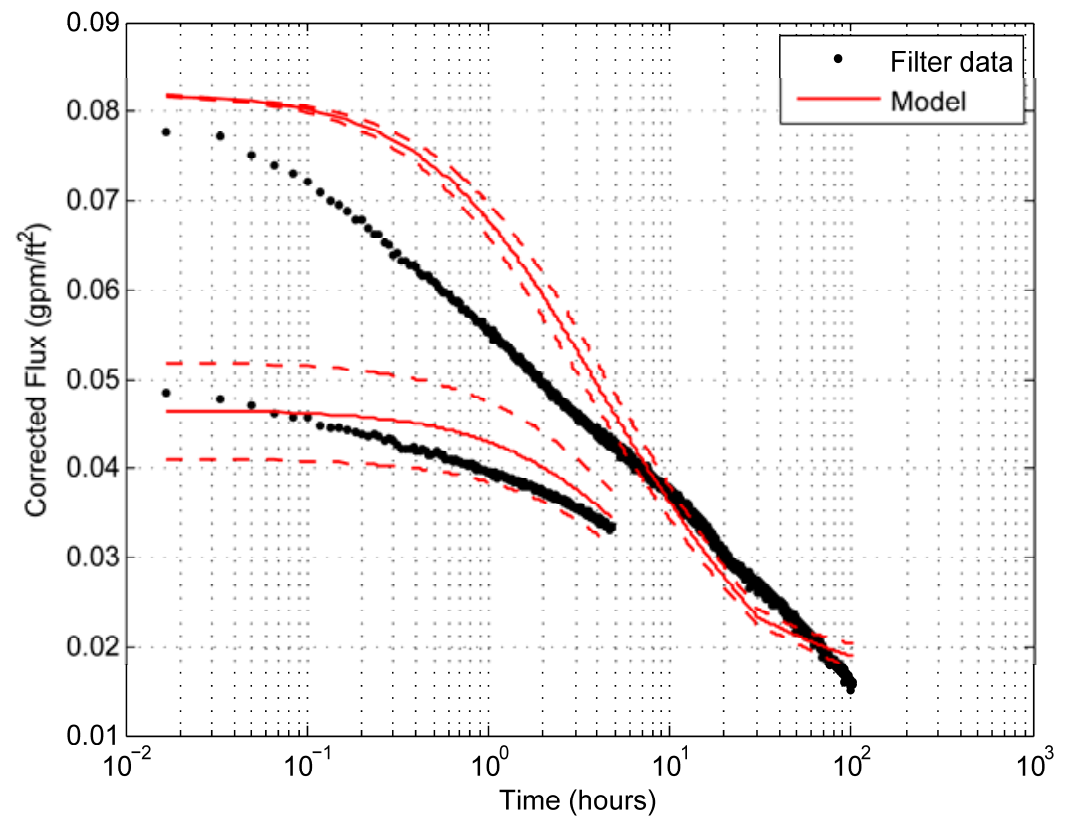

Figure A.17. Modified Model Fit to S1T2 Data Before Backpulsing and After Backpulsing Using Constraint in Equation 8.9. After backpulsing data is shown as beginning again at $t=0$ for clarity of presentation. The dotted lines are the bands of $95 \%$ confidence for the model fit. 


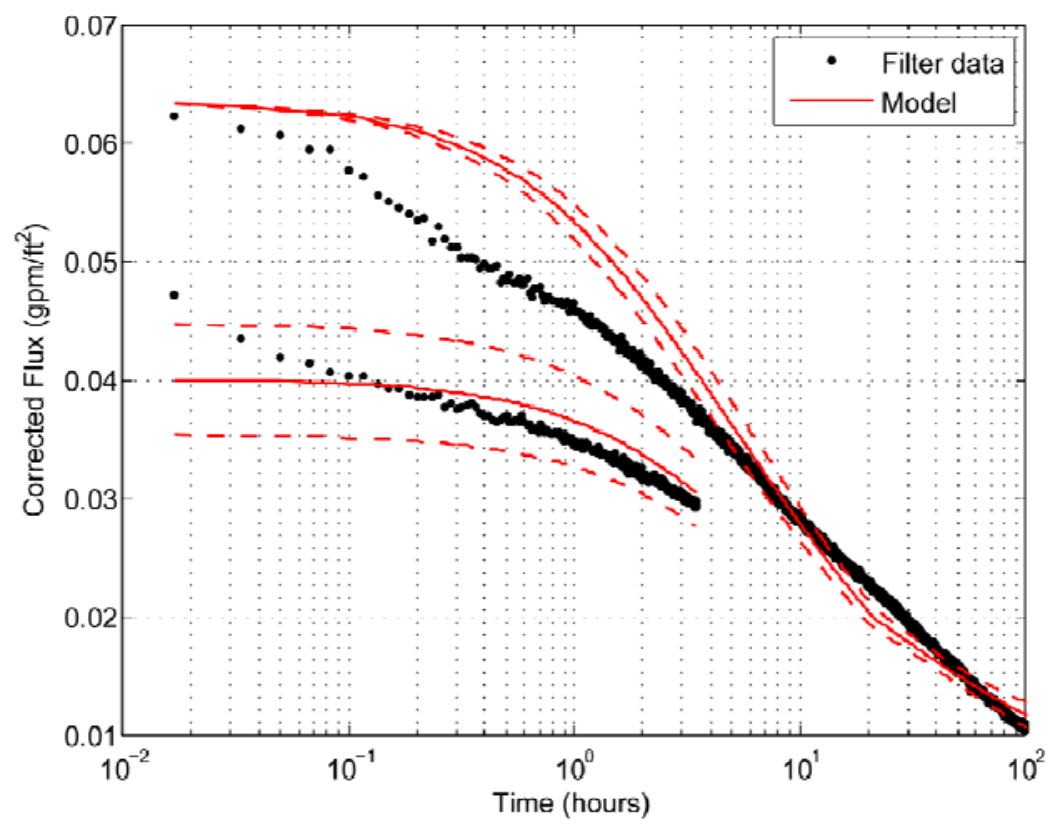

Figure A.18. Modified Model Fit to S1T3 Data Before Backpulsing and After Backpulsing Using Constraint in Equation 8.9. After backpulsing data is shown as beginning again at $t=0$ for clarity of presentation. The dotted lines are the bands of $95 \%$ confidence for the model fit.

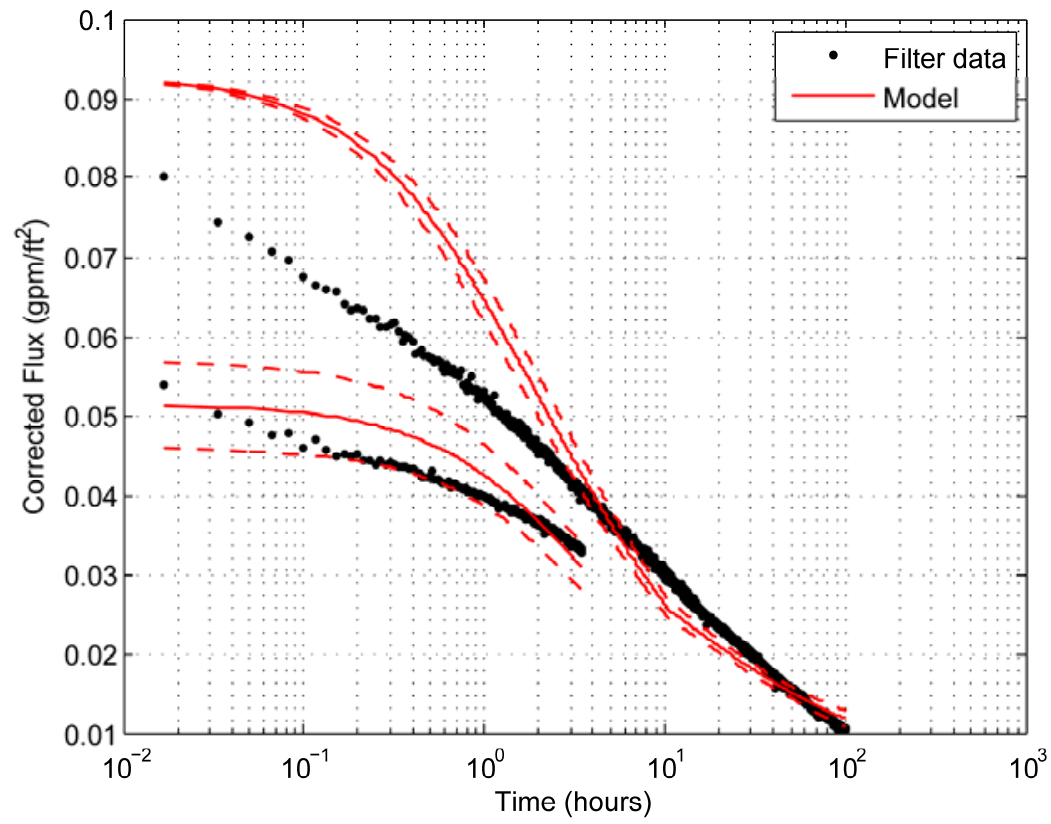

Figure A.19. Modified Model Fit to S1T4 Data Before Backpulsing and After Backpulsing Using Constraint in Equation 8.9. After backpulsing data is shown as beginning again at $t=0$ for clarity of presentation. The dotted lines are the bands of $95 \%$ confidence for the model fit. 


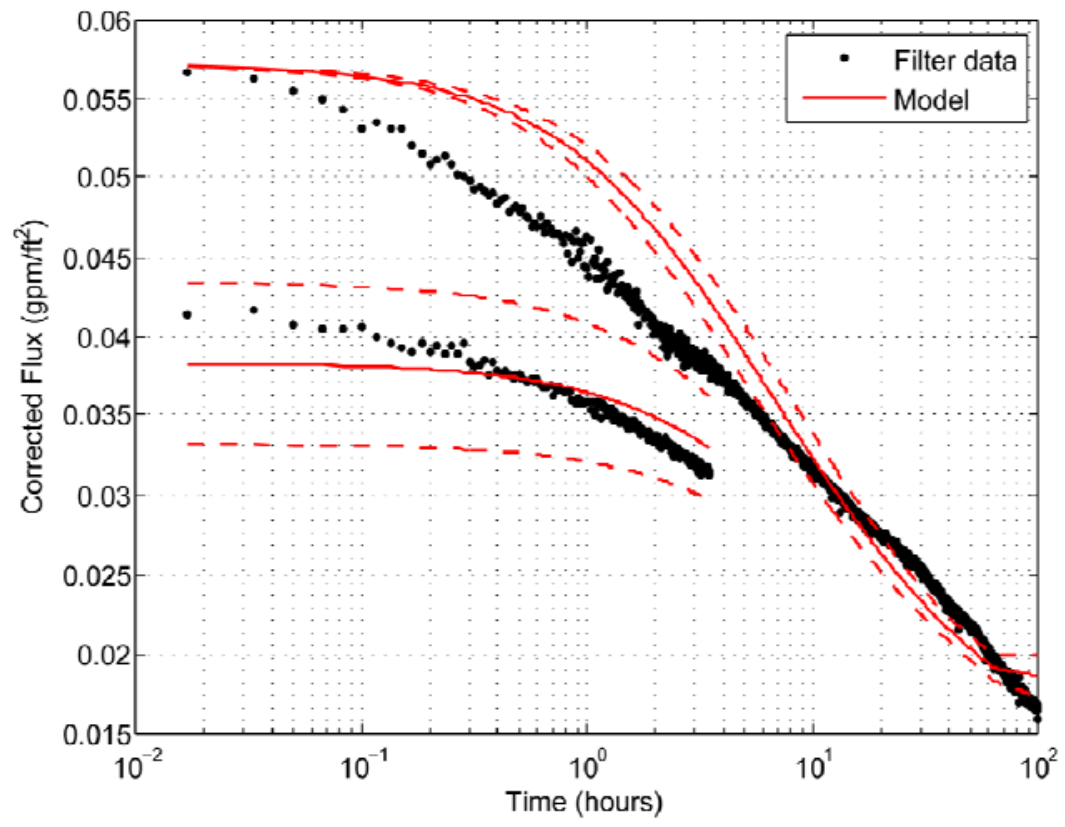

Figure A.20. Modified Model Fit to S1T5 Data Before Backpulsing and After Backpulsing Using Constraint in Equation 8.9. After backpulsing data is shown as beginning again at $t=0$ for clarity of presentation. The dotted lines are the bands of $95 \%$ confidence for the model fit. 


\section{A.4 Mechanistic Modeling: Flux Linearization Approach at Long-Times}

Table A.1. Root-Mean Squared Error for the Linearized Mechanism Fits to Each Series 1 Test for Three Time Periods: $t>10$ Hours, $t>25$ Hours, and $t>50$ Hours. The minimum RMSE for each data segment is bolded for convenience.

\begin{tabular}{|c|c|c|c|c|}
\hline Test & Mechanism & $\mathrm{t}>10$ hour & $\mathrm{t}>25$ hour & $\mathrm{t}>50$ hour \\
\hline \multirow{4}{*}{ S1T1 } & Pore & 5.202 & 2.665 & 1.686 \\
\hline & Constriction & 3.454 & 1.873 & 1.475 \\
\hline & Intermediate & 1.887 & 1.308 & 1.320 \\
\hline & Cake & 2.535 & 1.855 & 1.251 \\
\hline \multirow{4}{*}{ S1T2 } & Pore & 6.036 & 1.997 & 1.267 \\
\hline & Constriction & 4.144 & 1.338 & 1.240 \\
\hline & Intermediate & 2.562 & 1.337 & 1.330 \\
\hline & Cake & 3.259 & 2.886 & 1.772 \\
\hline \multirow{4}{*}{ S1T3 } & Pore & 4.062 & 2.261 & 1.309 \\
\hline & Constriction & 2.568 & 1.527 & 1.112 \\
\hline & Intermediate & 1.205 & 0.953 & 0.956 \\
\hline & Cake & 2.243 & 1.428 & 0.855 \\
\hline \multirow{4}{*}{ S1T4 } & Pore & 4.783 & 2.688 & 1.324 \\
\hline & Constriction & 3.166 & 1.895 & 1.157 \\
\hline & Intermediate & 1.643 & 1.221 & 1.048 \\
\hline & Cake & 2.100 & 1.378 & 1.068 \\
\hline \multirow{4}{*}{ S1T5 } & Pore & 4.696 & 2.962 & 2.171 \\
\hline & Constriction & 3.377 & 2.288 & 1.956 \\
\hline & Intermediate & 2.181 & 1.732 & 1.769 \\
\hline & Cake & 1.795 & 1.546 & 1.513 \\
\hline
\end{tabular}



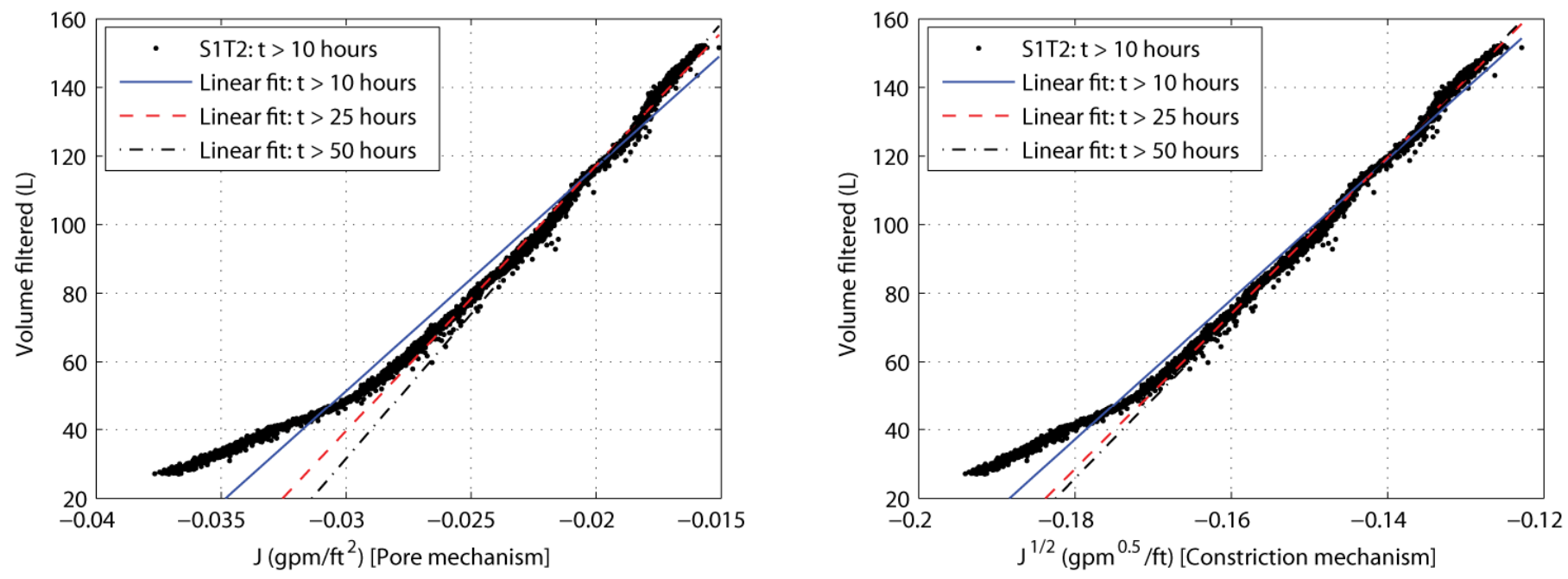

$\stackrel{p}{\omega}$
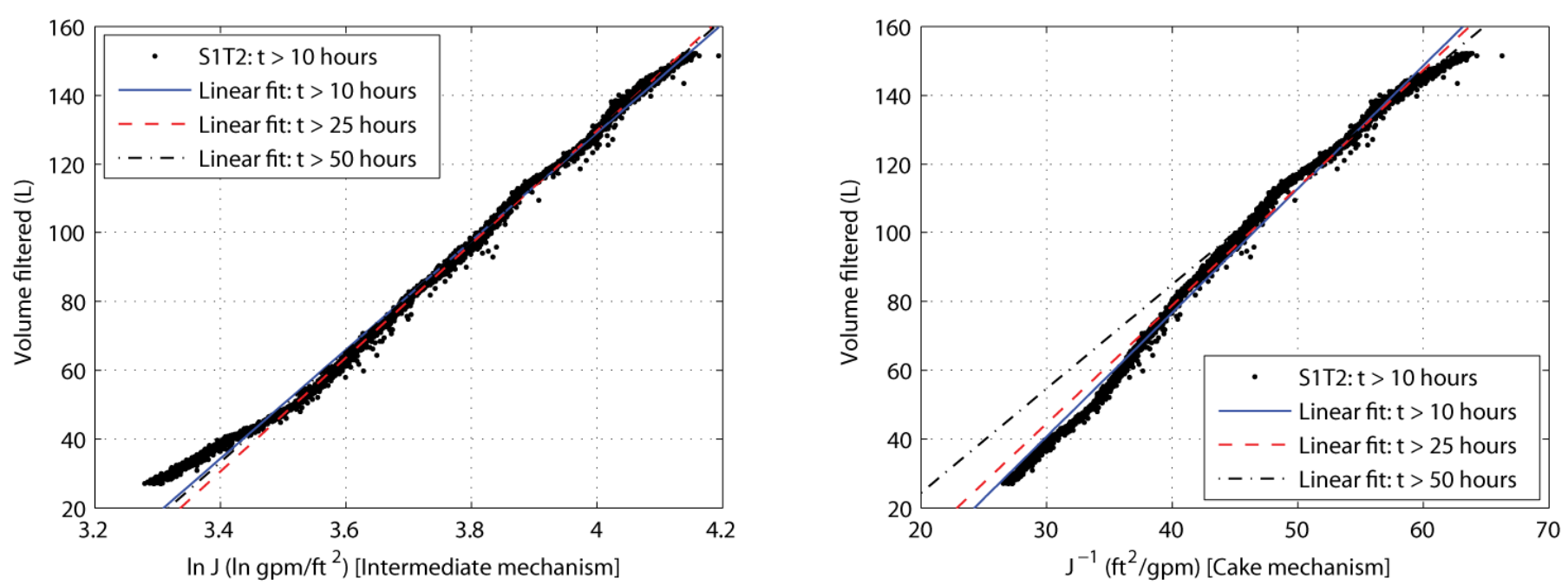

Figure A.21. Linearized Blocking Laws for S1T2 at Long-Times: $\mathrm{f}(J)$ Versus $V$, with Linear Regression Applied for Data from $t>10, t>25$, and $t>50$ Hours. Clockwise from top left: pore, constriction, cake and intermediate blocking laws, respectively. 

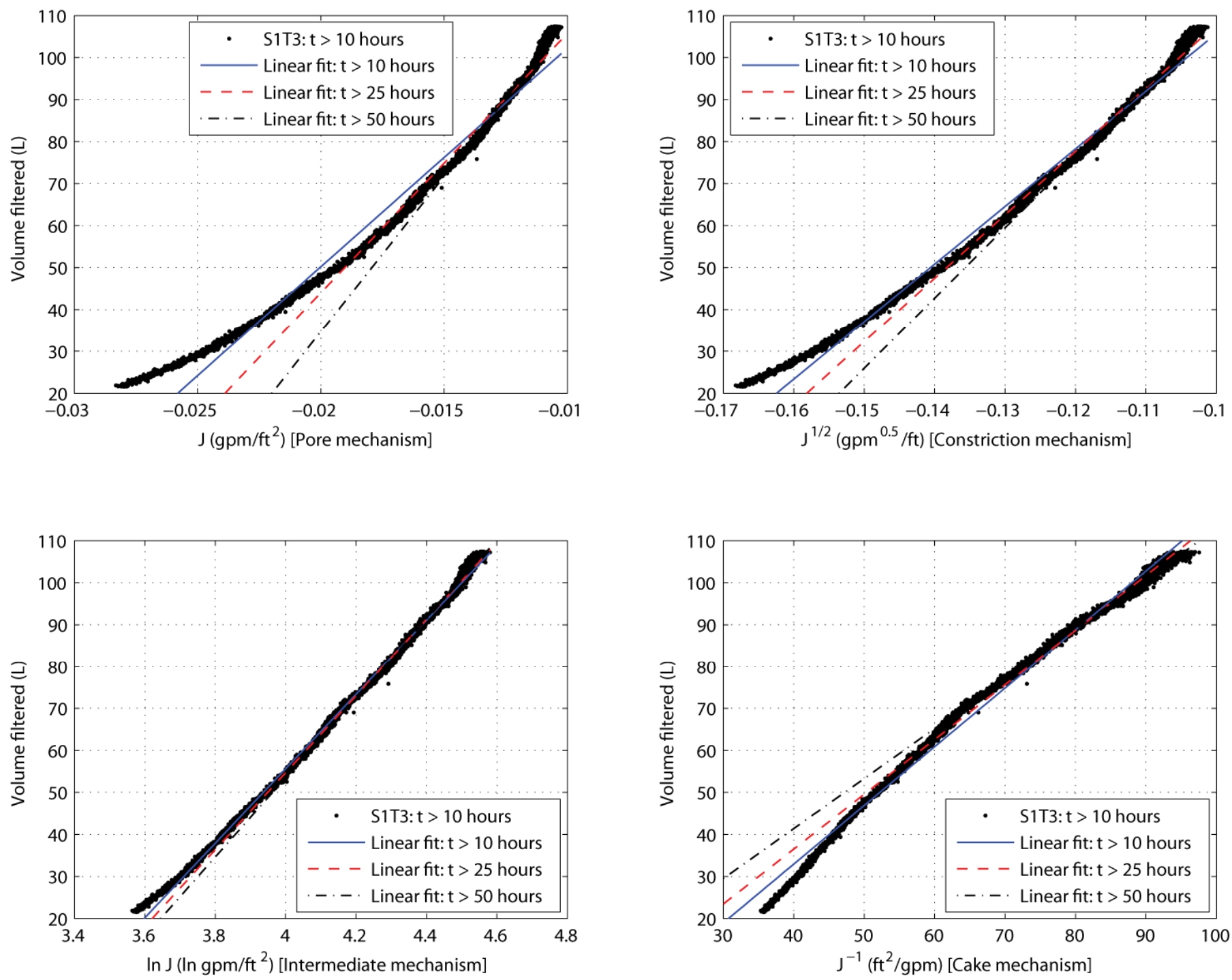

Figure A.22. Linearized Blocking Laws for S1T3 at Long-Times: $\mathrm{f}(J)$ Versus $V$, with Linear Regression Applied for Data from $t>10, t>25$, and $t>50$ Hours. Clockwise from top left: pore, constriction, cake and intermediate blocking laws, respectively. 

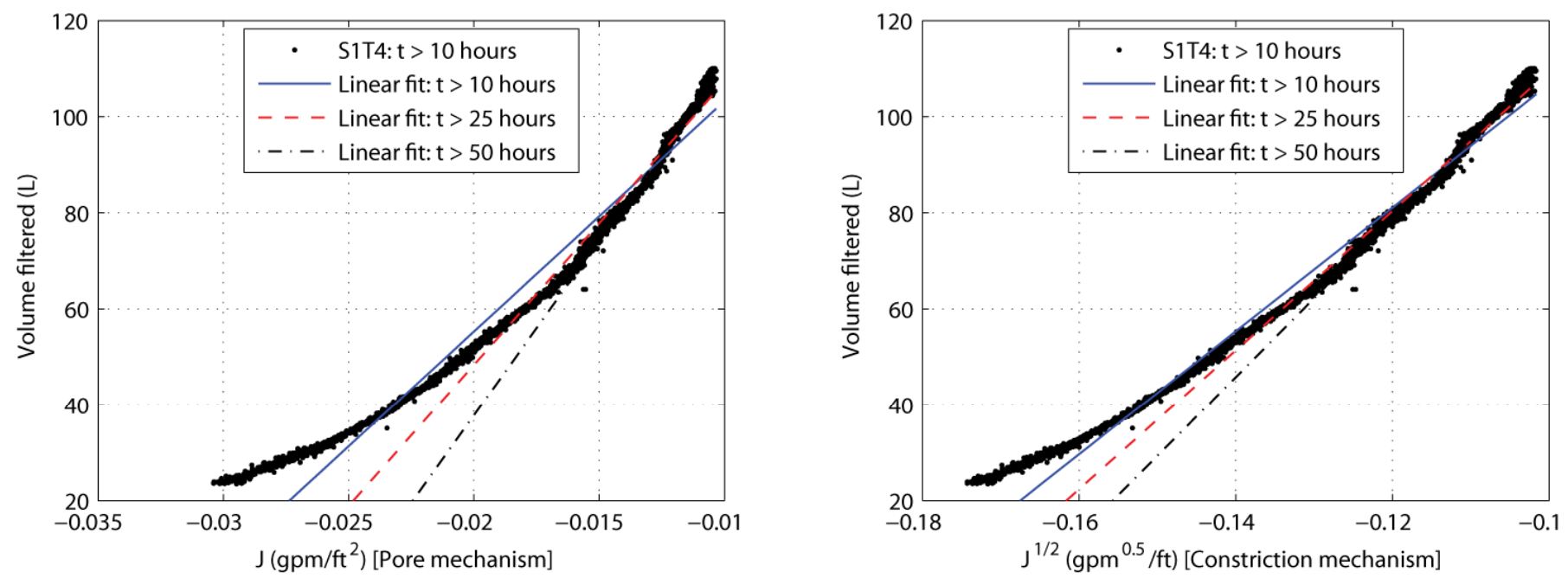

$\frac{p}{u}$
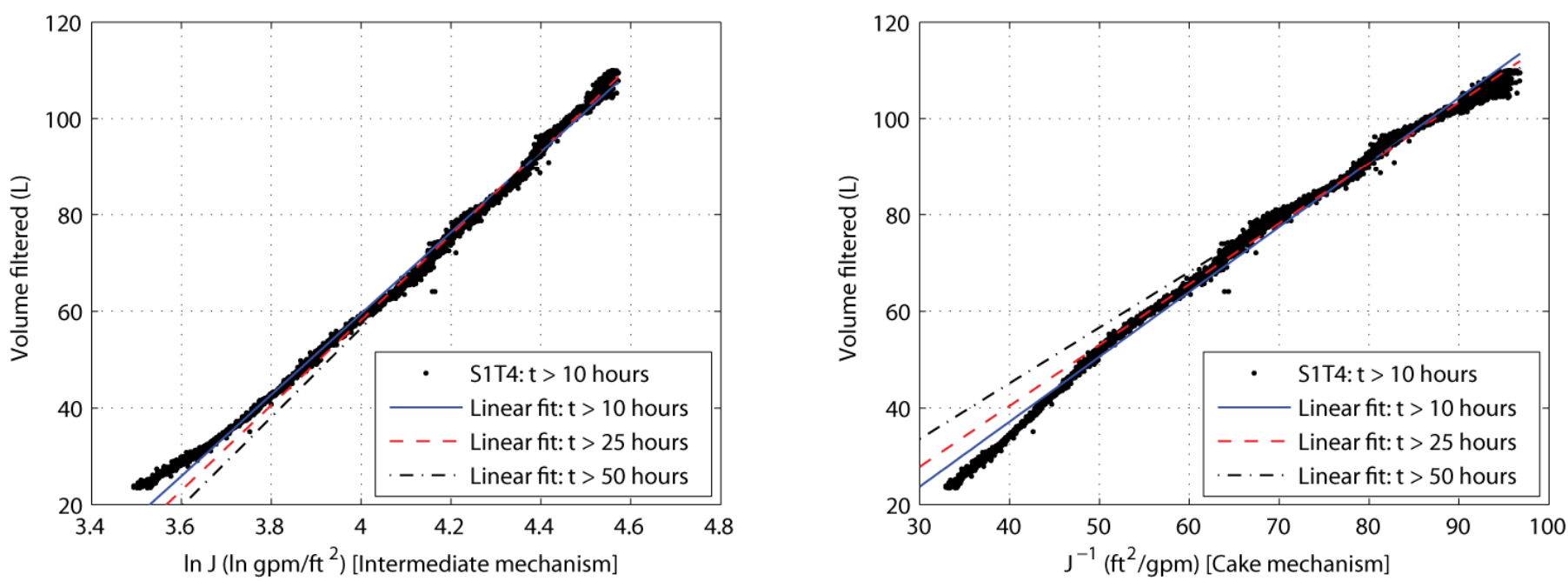

Figure A.23. Linearized Blocking Laws for S1T4 at Long-Times: $\mathrm{f}(J)$ Versus $V$, with Linear Regression Applied for Data from $t>10, t>25$, and $t>50$ Hours. Clockwise from top left: pore, constriction, cake and intermediate blocking laws, respectively. 

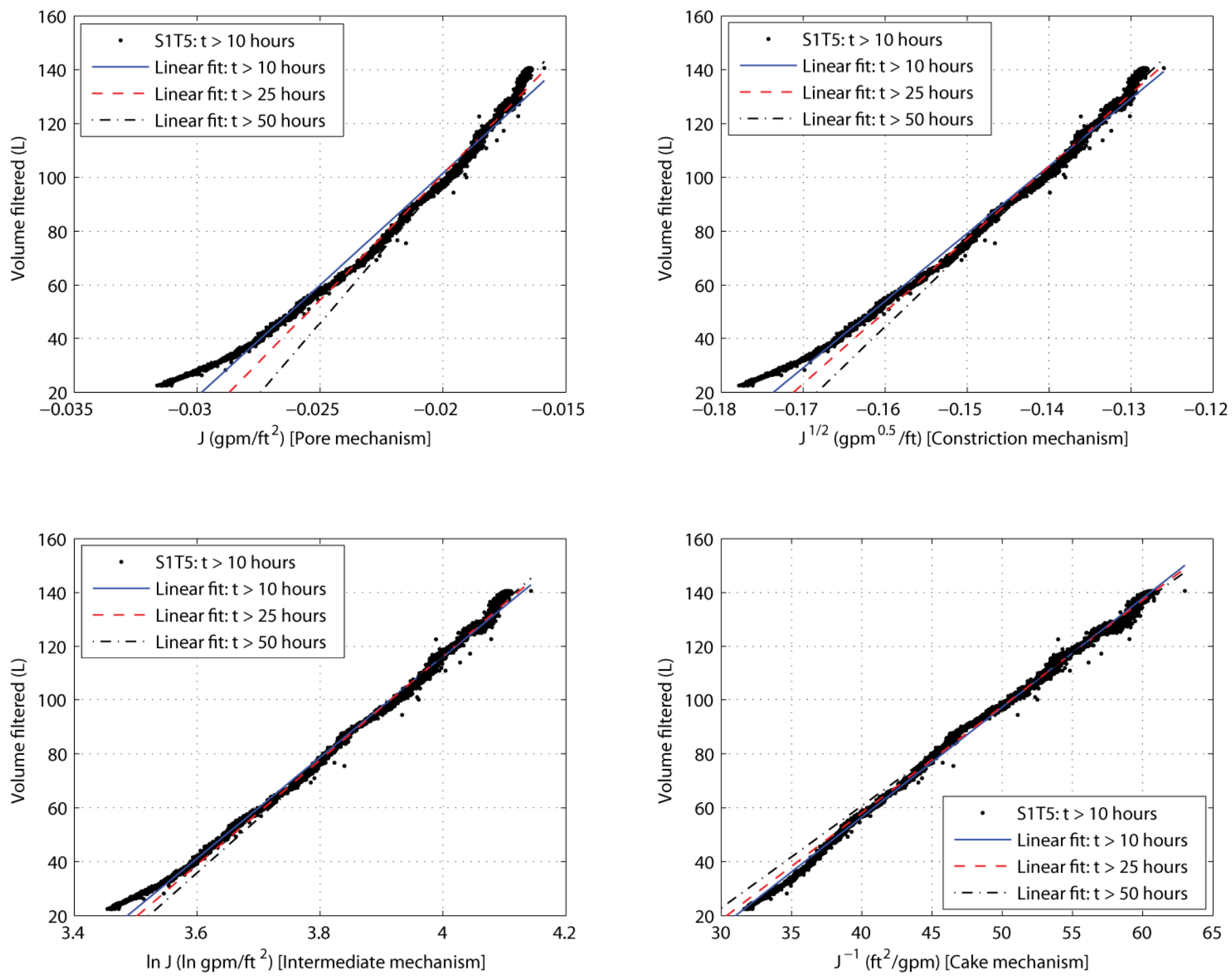

Figure A.24. Linearized Blocking Laws for S1T5 at Long-Times: $\mathrm{f}(J)$ Versus $V$, with Linear Regression Applied for Data from $t>10, t>25$, and $t>50$ Hours. Clockwise from top left: pore, constriction, cake and intermediate blocking laws, respectively. 


\section{A.5 Mechanistic Modeling: Flux Linearization Approach at Intermediate Times}

Table A.2. Root-Mean Squared Error for the Linearized Mechanism Fits to Each Series 1 test for Three Time Periods: $5<\mathrm{t}<10$ Hours, $5<\mathrm{t}<15$ Hours, and $5<\mathrm{t}<20$ Hours. The minimum RMSE for each data segment is bolded for convenience.

\begin{tabular}{|c|c|c|c|c|}
\hline Test & Mechanism & $5<\mathrm{t}<10$ hour & $5<\mathrm{t}<15$ hour & $5<\mathrm{t}<20$ hour \\
\hline \multirow{4}{*}{ S1T1 } & Pore & 1.323 & 1.449 & 1.624 \\
\hline & Constriction & 1.313 & 1.397 & 1.479 \\
\hline & Intermediate & 1.305 & 1.356 & 1.355 \\
\hline & Cake & 1.295 & 1.304 & 1.193 \\
\hline \multirow{4}{*}{ S1T2 } & Pore & 0.681 & 0.861 & 0.949 \\
\hline & Constriction & 0.669 & 0.815 & 0.848 \\
\hline & Intermediate & 0.659 & 0.782 & 0.793 \\
\hline & Cake & 0.643 & 0.756 & 0.850 \\
\hline \multirow{4}{*}{ S1T3 } & Pore & 0.371 & 0.737 & 1.174 \\
\hline & Constriction & 0.355 & 0.650 & 1.004 \\
\hline & Intermediate & 0.343 & 0.567 & 0.839 \\
\hline & Cake & 0.325 & 0.433 & 0.551 \\
\hline \multirow{4}{*}{ S1T4 } & Pore & 0.443 & 0.617 & 0.968 \\
\hline & Constriction & 0.436 & 0.556 & 0.790 \\
\hline & Intermediate & 0.433 & 0.515 & 0.633 \\
\hline & Cake & 0.437 & 0.512 & 0.472 \\
\hline \multirow{4}{*}{ S1T5 } & Pore & 0.386 & 0.795 & 1.335 \\
\hline & Constriction & 0.369 & 0.730 & 1.205 \\
\hline & Intermediate & 0.354 & 0.668 & 1.077 \\
\hline & Cake & 0.327 & 0.556 & 0.834 \\
\hline
\end{tabular}



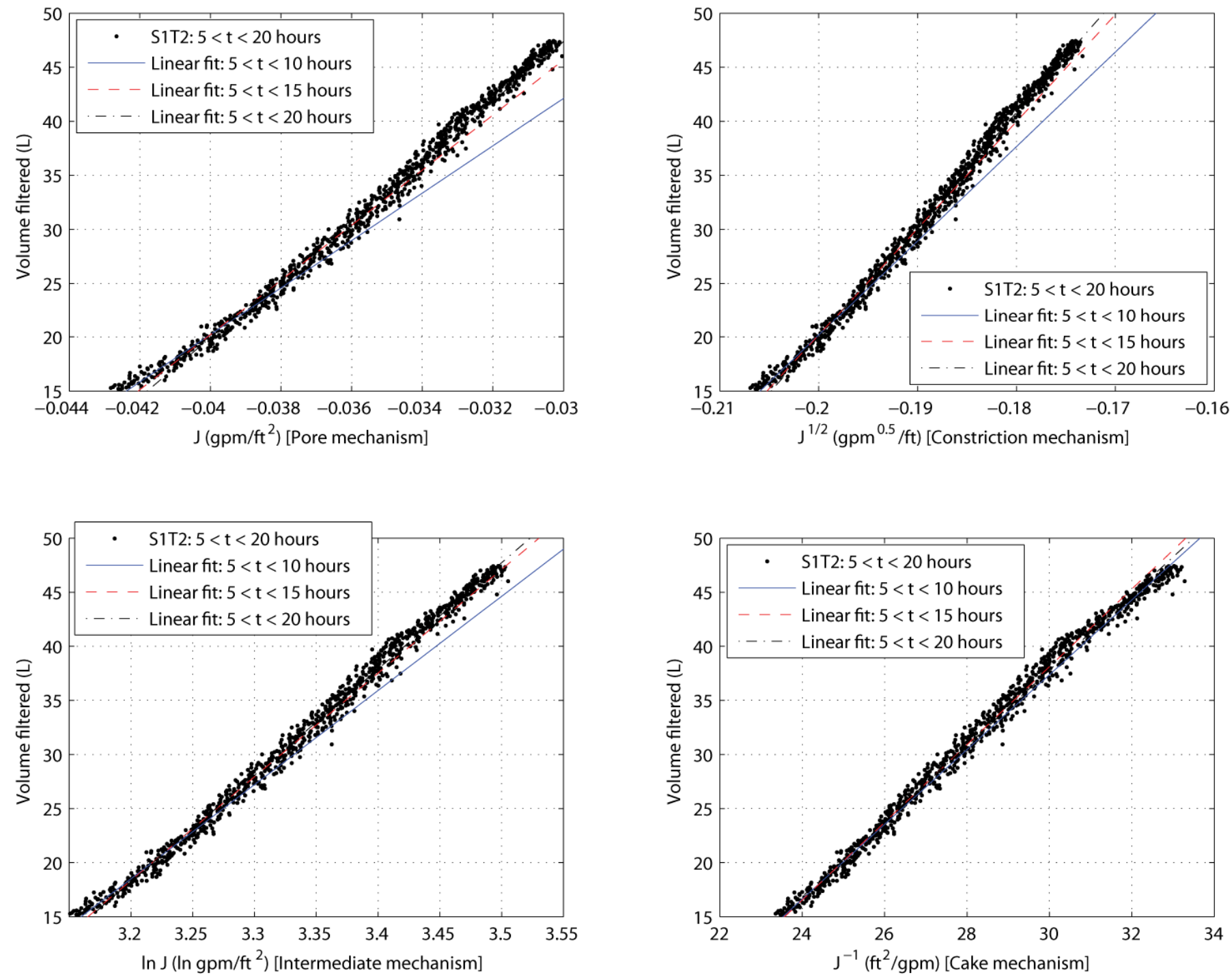

Figure A.25. Linearized Blocking Laws for S1T2 at Intermediate Times: $\mathrm{f}(J)$ Versus $V$, with Linear Regression Applied for Data from $5<t<10,5<t<15$, and $5<t<20$ Hours. Clockwise from top left: pore, constriction, cake and intermediate blocking laws, respectively. 

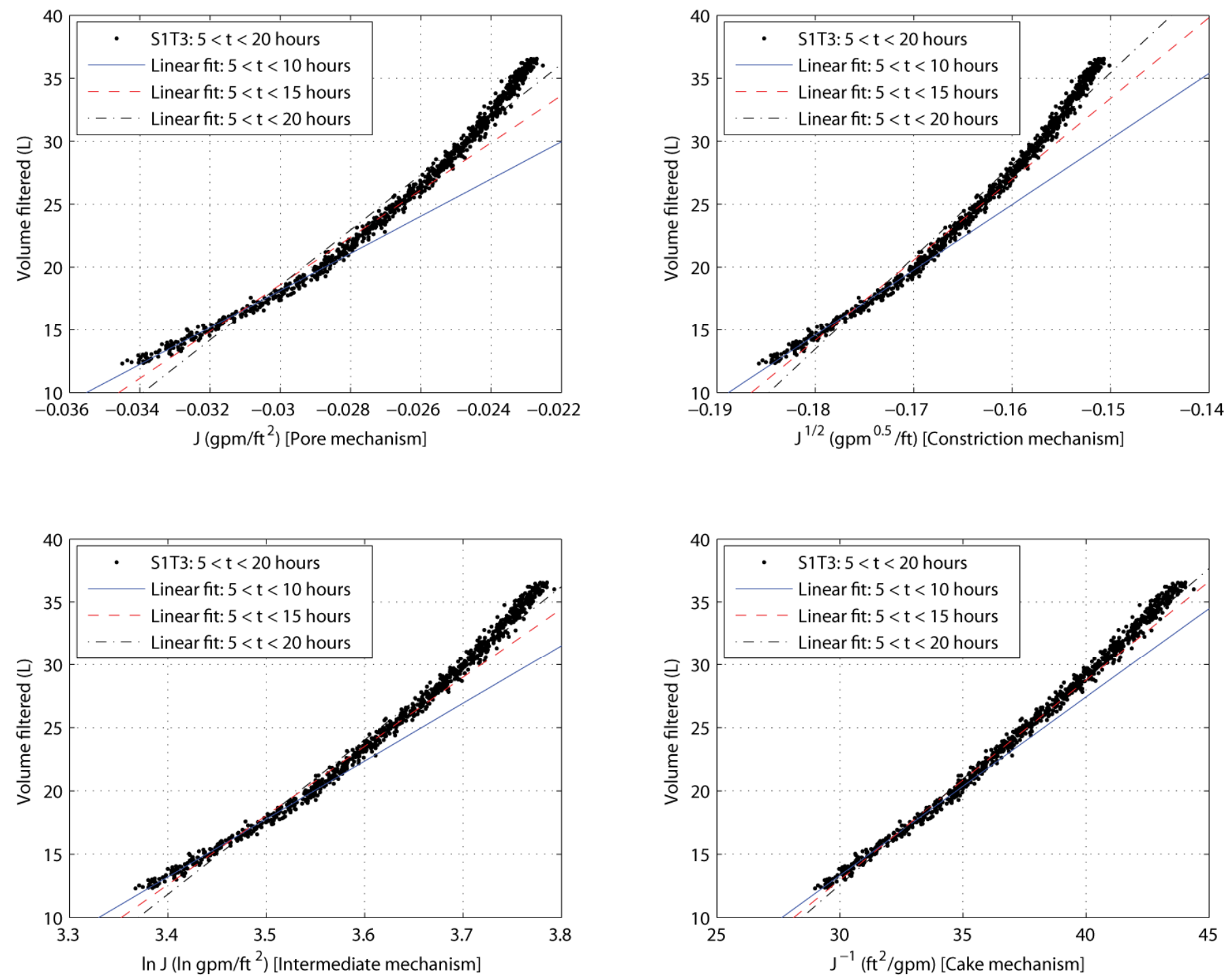

Figure A.26. Linearized Blocking Laws for S1T3 at Intermediate Times: $\mathrm{f}(J)$ Versus $V$, with Linear Regression Applied for Data from $5<t<10,5<t<15$, and $5<t<20$ Hours. Clockwise from top left: pore, constriction, cake and intermediate blocking laws, respectively. 

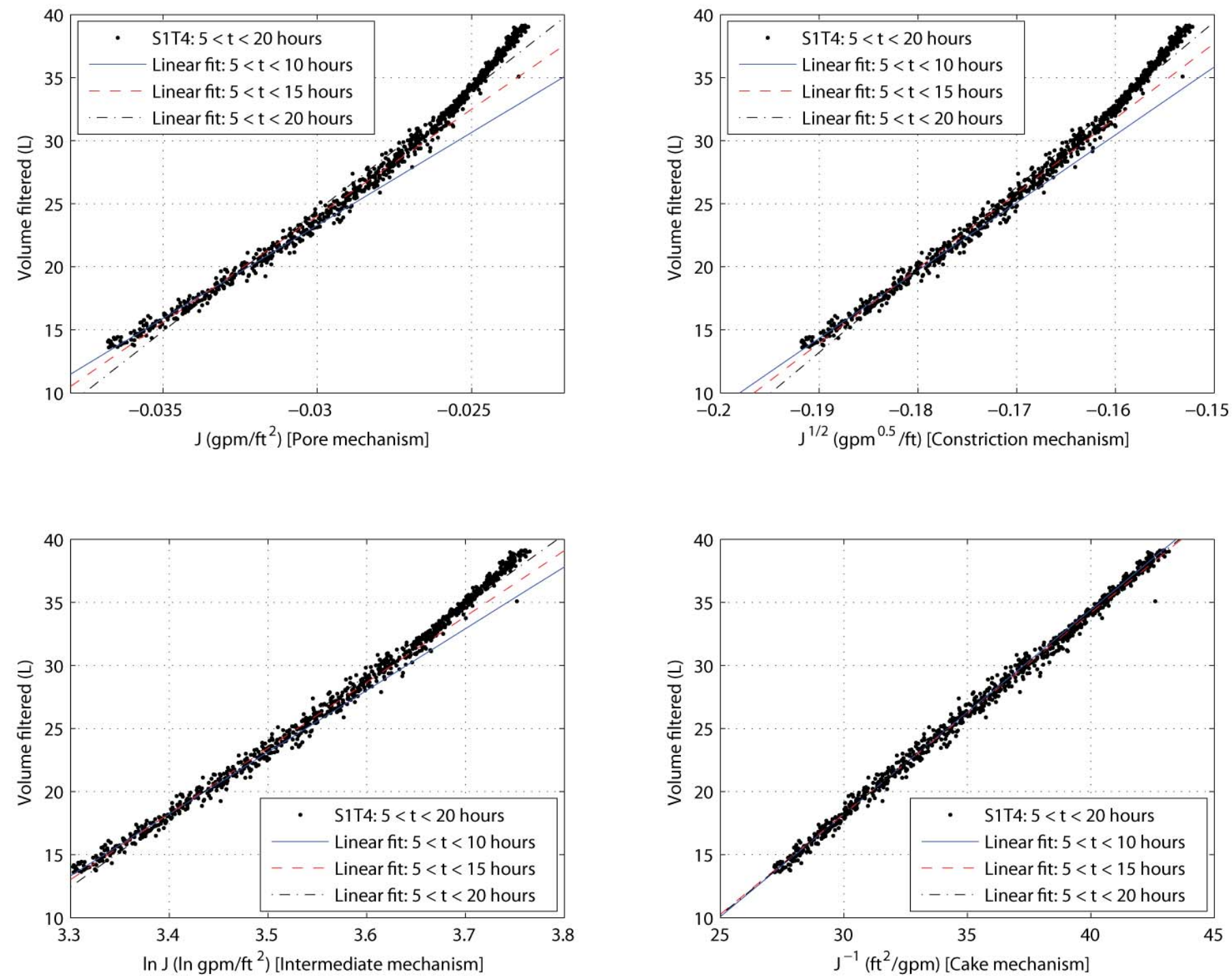

Figure A.27. Linearized Blocking Laws for S1T4 at Intermediate Times: $\mathrm{f}(J)$ Versus $V$, with Linear Regression Applied for Data from $5<t<10,5<t<15$, and $5<t<20$ Hours. Clockwise from top left: pore, constriction, cake and intermediate blocking laws, respectively. 

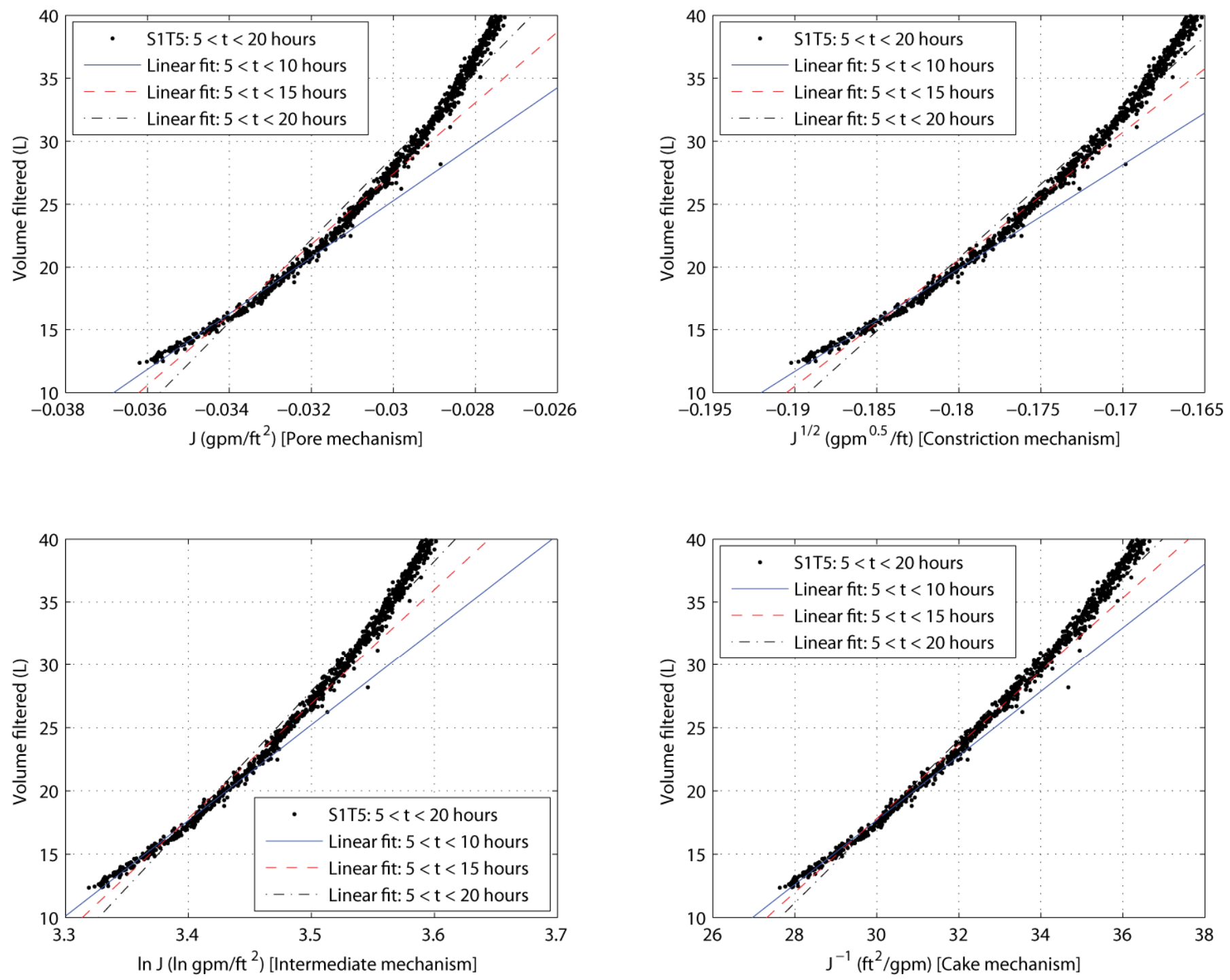

Figure A.28. Linearized Blocking Laws for S1T5 at Intermediate Times: $\mathrm{f}(J)$ Versus $V$, with Linear Regression Applied for Data From $5<t<10,5<t<15$, and $5<t<20$ Hours. Clockwise from top left: pore, constriction, cake and intermediate blocking laws, respectively. 


\section{A.6 Mechanistic Modeling: Optimization for Intermediate Mechanism at Long-Times}

An example of an error plot from which the optimum was selected is given in Figure A.29. An example of the normal probability plot for the residuals of the optimum fit is given in Figure A.30. These figures correspond to the S1T1 figures described in Section 8.2 (see Figure 8.13 and Figure 8.14). What follows is the model fits for the other four tests (S1T2 - S1T5) in both shifted coordinates $\left(J / J_{1}, \tau_{1}\right)$ and the original coordinates $(\mathrm{J}(\mathrm{m} / \mathrm{s}), t(\mathrm{hr}))$. The error plot and normal probability plot are not supplied for these four tests but they are similar in nature to Figure A.29 and Figure A.30.

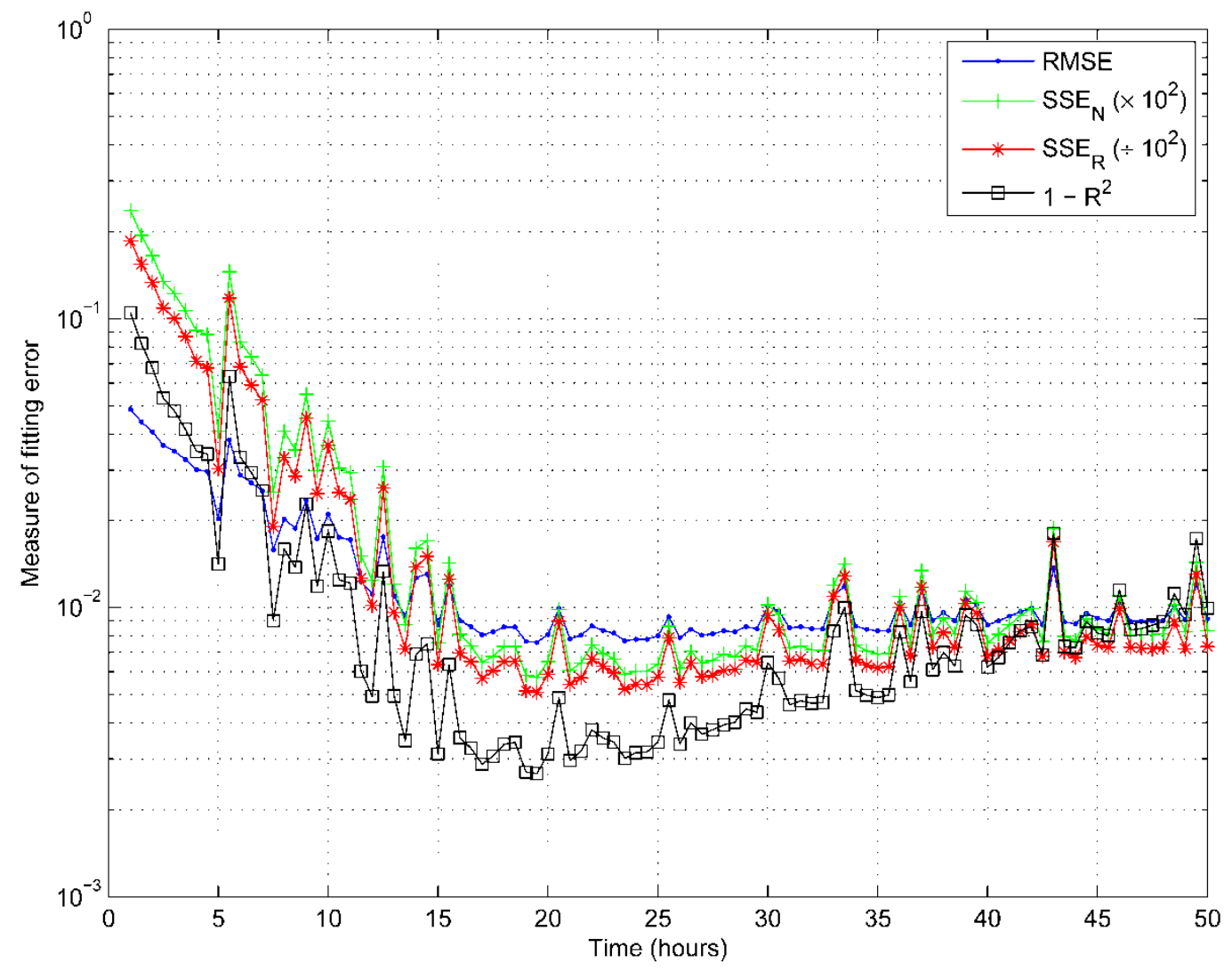

Figure A.29. Goodness-of-Fit Statistics for the Long-Time Intermediate Mechanism Model when Fit to the S1T1 Data. The statistics are plotted as a function of the time where the intermediate mechanism is assumed to first apply $\left(t_{1}\right): \mathrm{RMSE}=$ root-mean squared error, $\mathrm{SSE}_{\mathrm{N}}=$ sum of squares error normalized by number of data points, $\mathrm{SSE}_{\mathrm{R}}=$ sum of squares error normalized by data range, and $\mathrm{R}^{2}=$ coefficient of determination. 


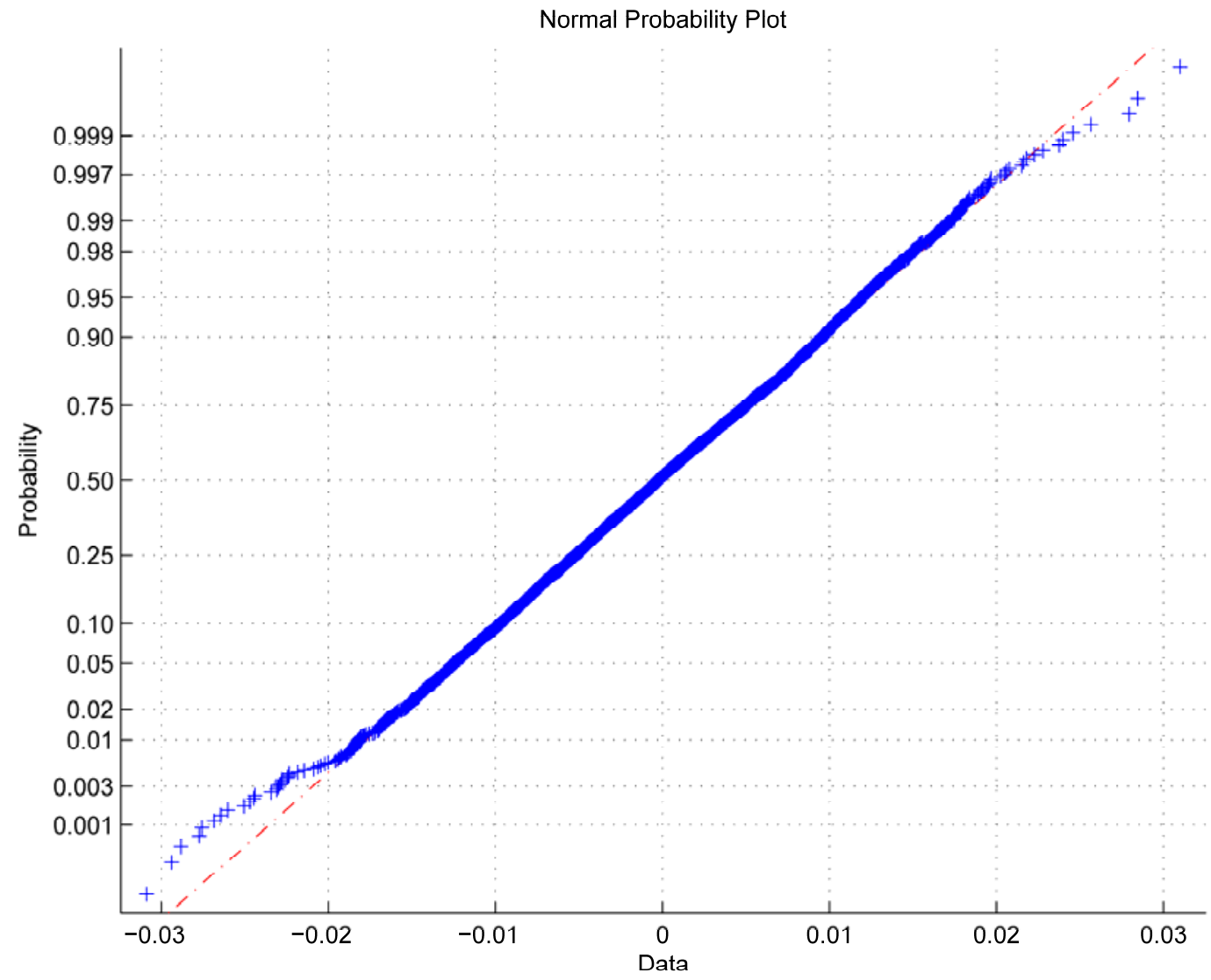

Figure A.30. Normal Probability Plot for the Residuals of the Optimum Fit to the S1T1 Data Using the Intermediate Mechanism. The residuals from the fit are given as blue '+', and the red dot-dash line is the expected behavior if the residuals are normally distributed with a mean of zero. 


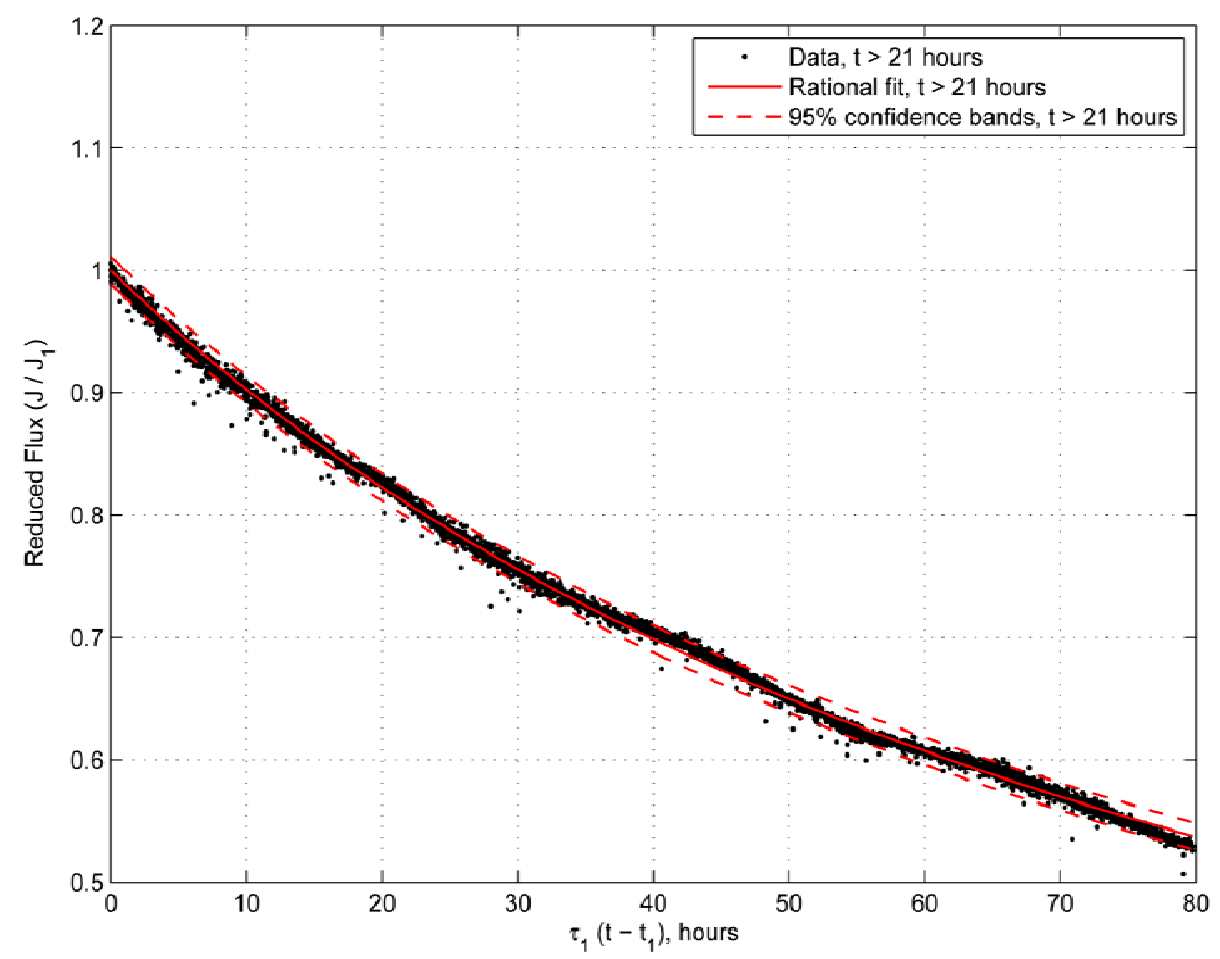

Figure A.31. Optimum Fit to S1T2 Flux-Time Data Using the Model of Equation 8.23. The bands of 95\% confidence for the fit are shown as dotted lines.

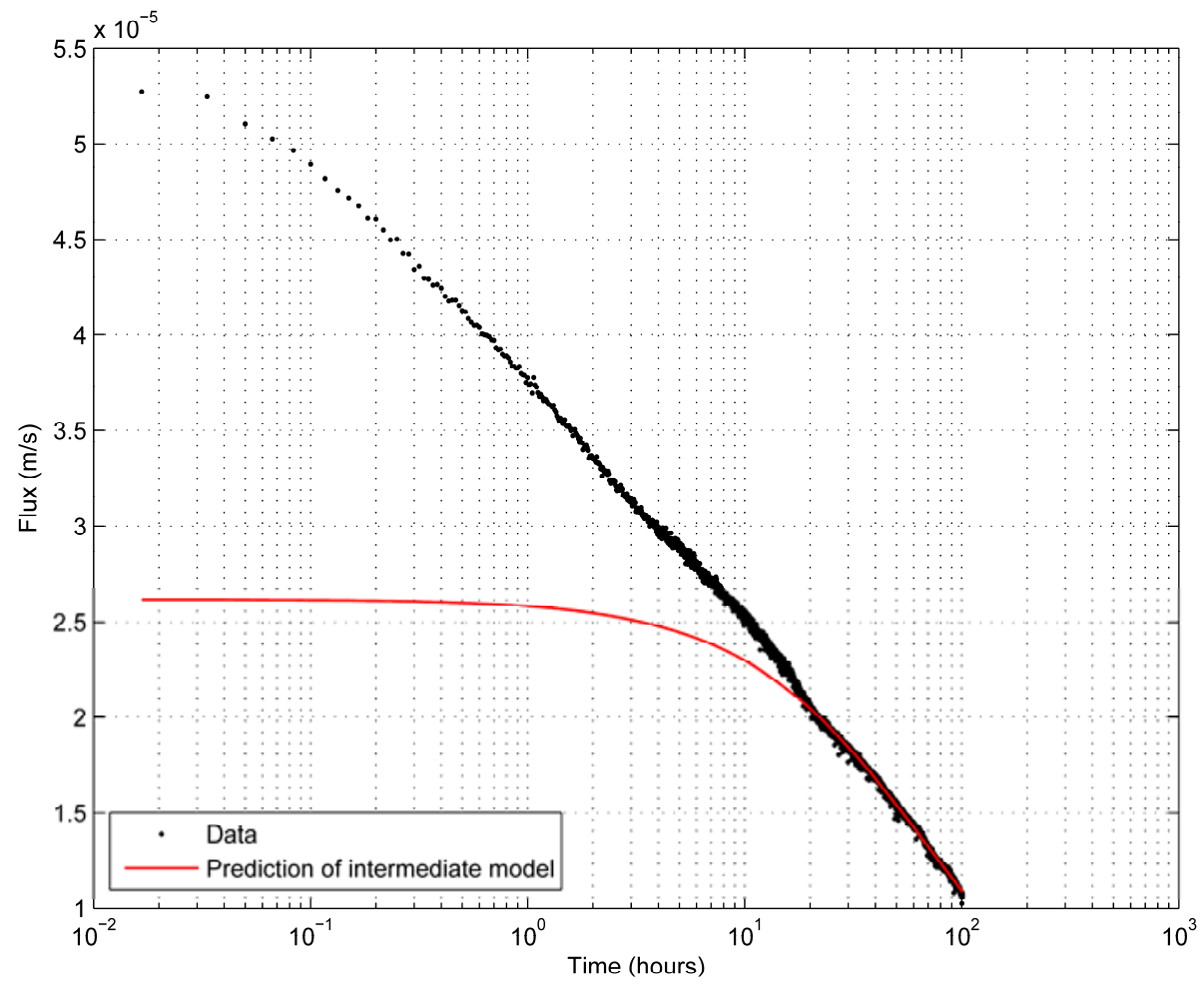

Figure A.32. Long Time Optimum Fit for S1T2 Data Using the Intermediate Mechanism Model 


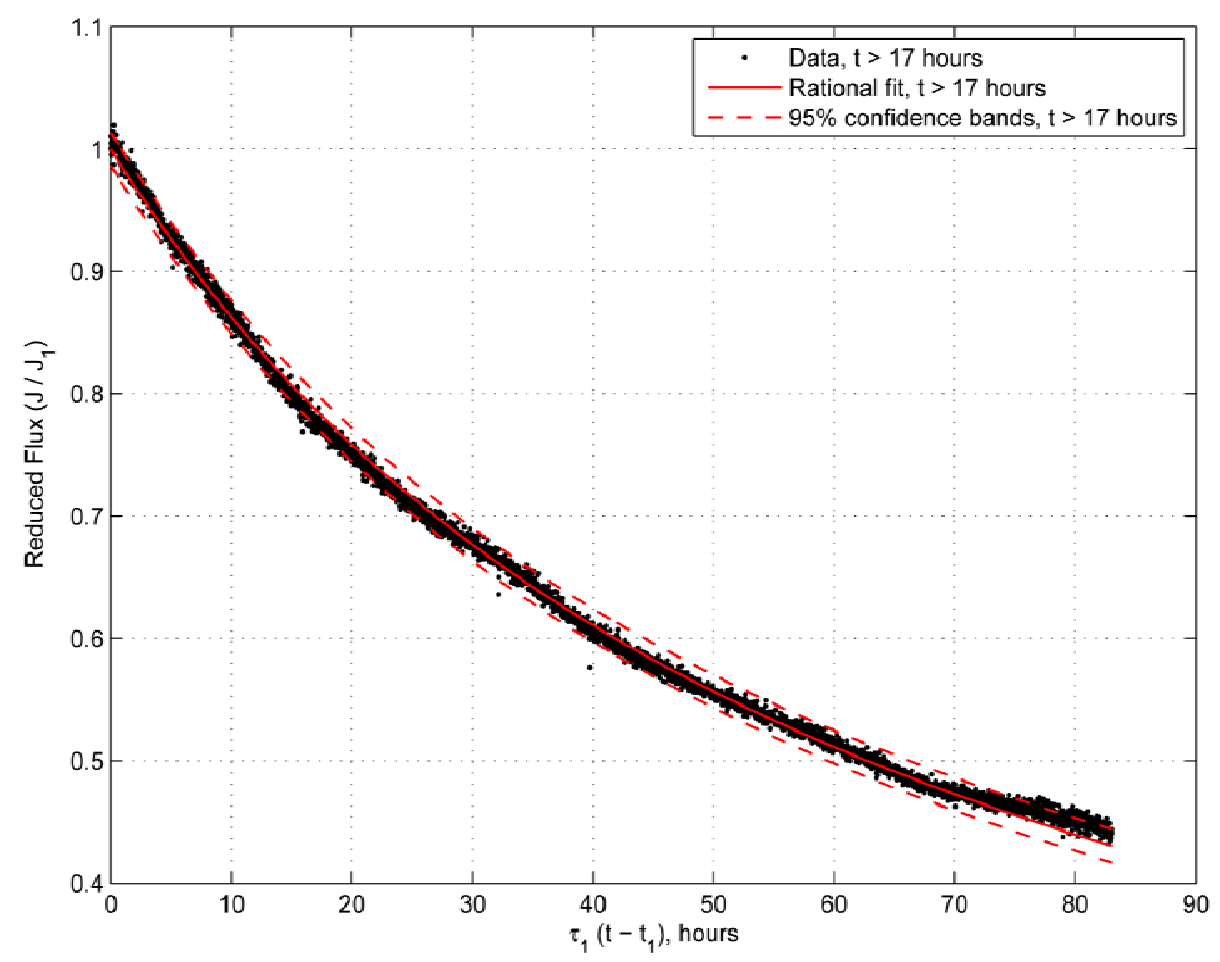

Figure A.33. Optimum Fit to S1T3 Flux-Time Data Using the Model of Equation 8.23. The bands of 95\% confidence for the fit are shown as dotted lines.

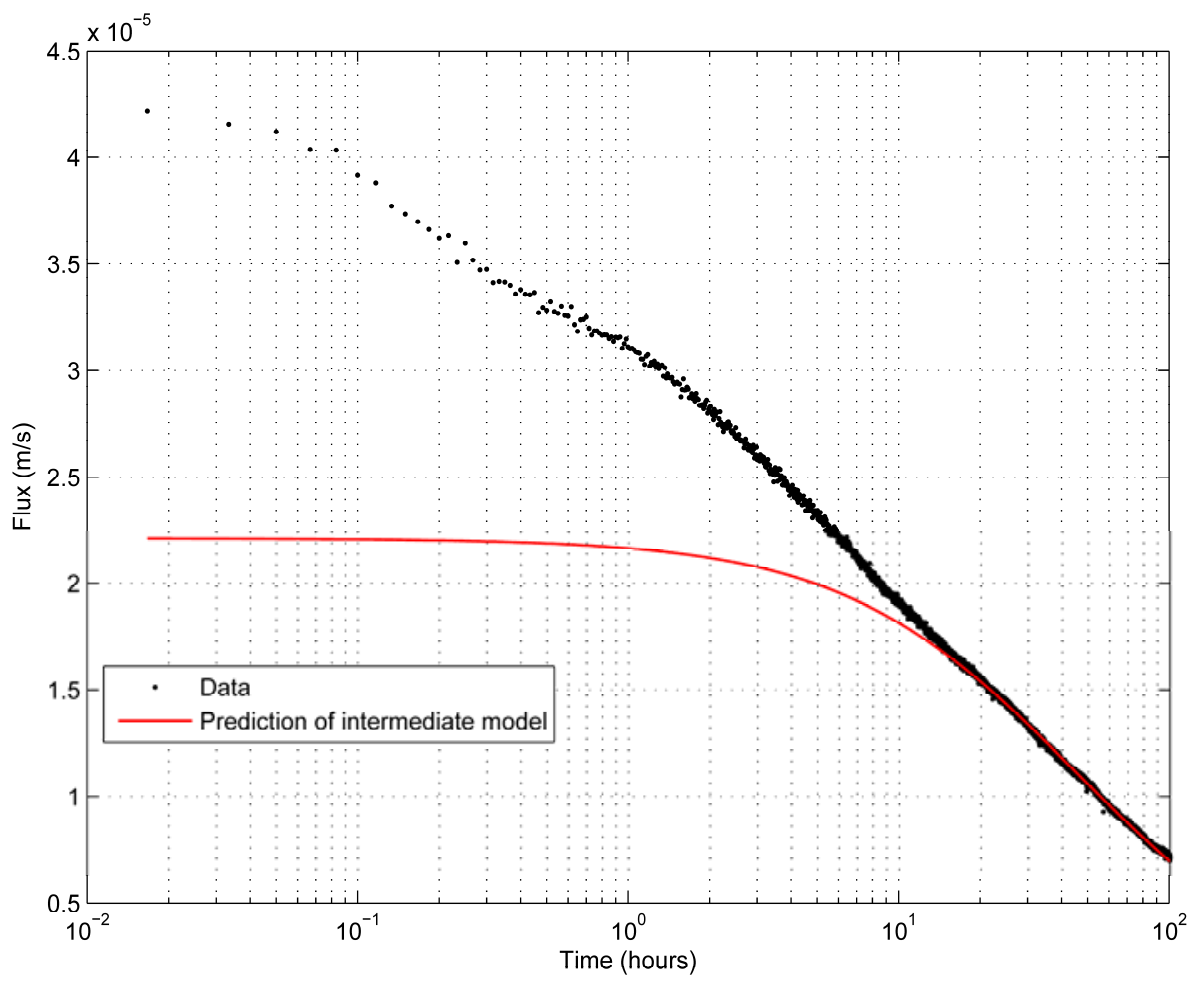

Figure A.34. Long Time Optimum Fit for S1T3 Data Using the Intermediate Mechanism Model 


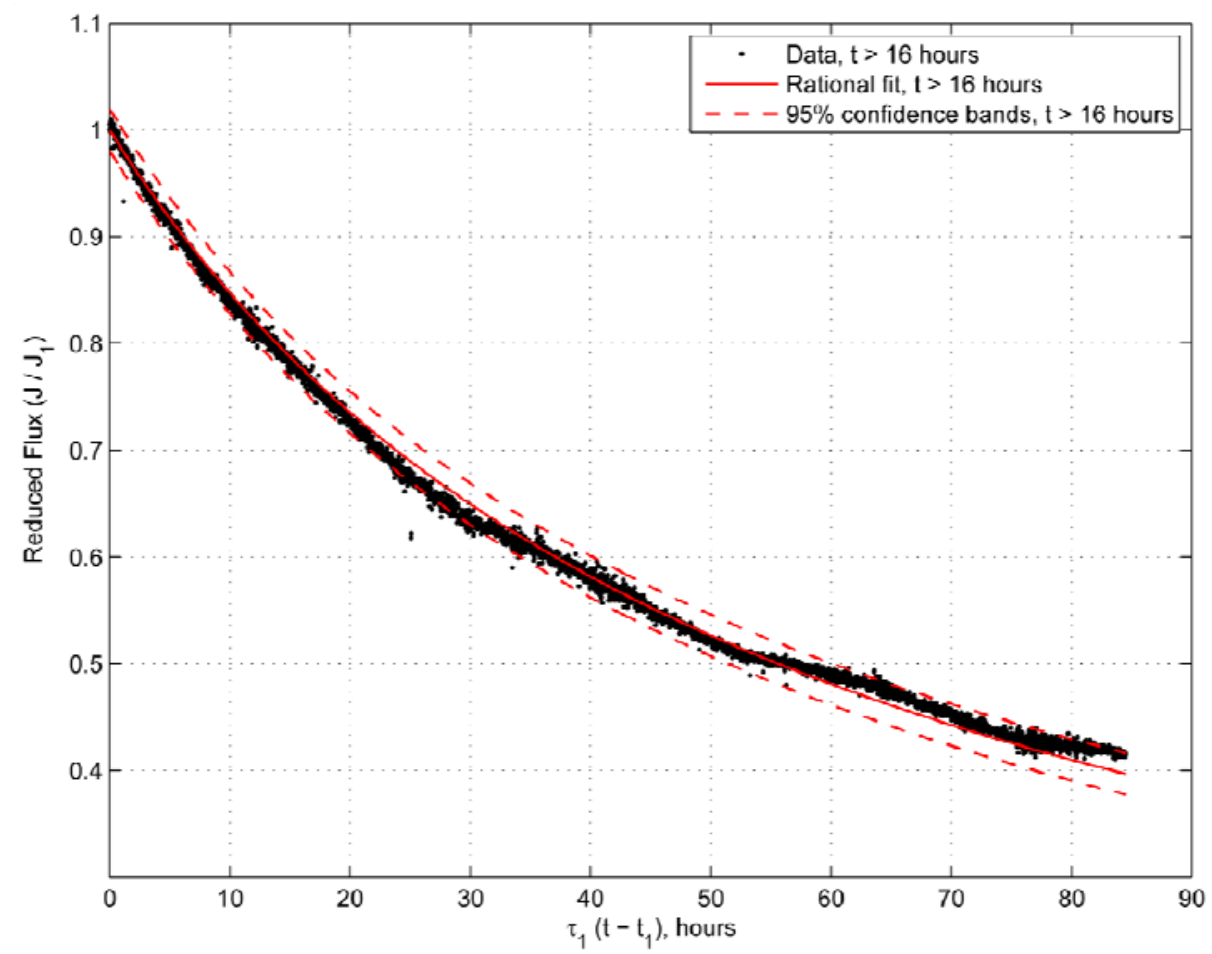

Figure A.35. Optimum Fit to S1T4 Flux-Time Data Using the Model of Equation 8.23. The bands of 95\% confidence for the fit are shown as dotted lines.

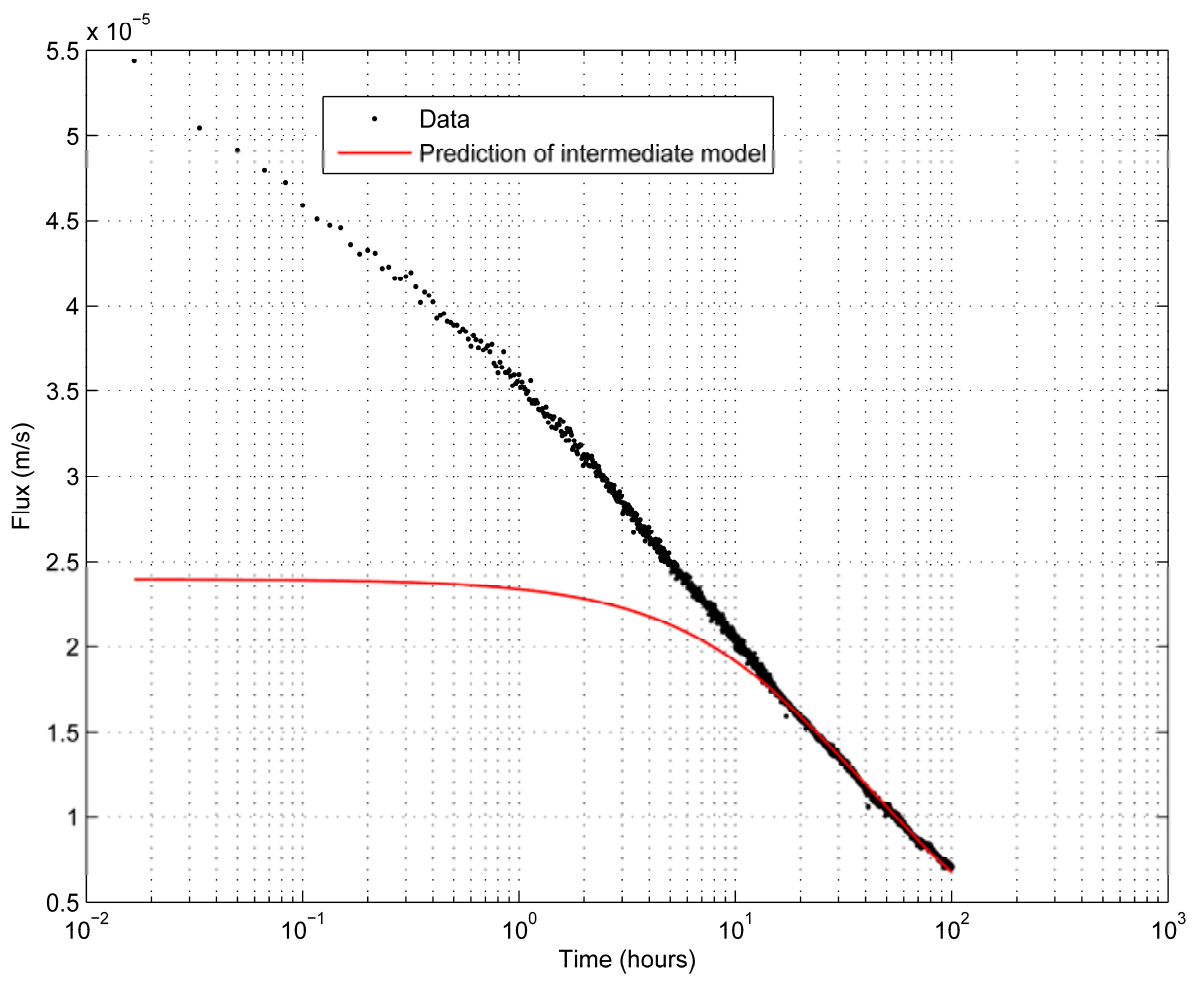

Figure A.36. Long Time Optimum Fit for S1T4 Data Using the Intermediate Mechanism Model 


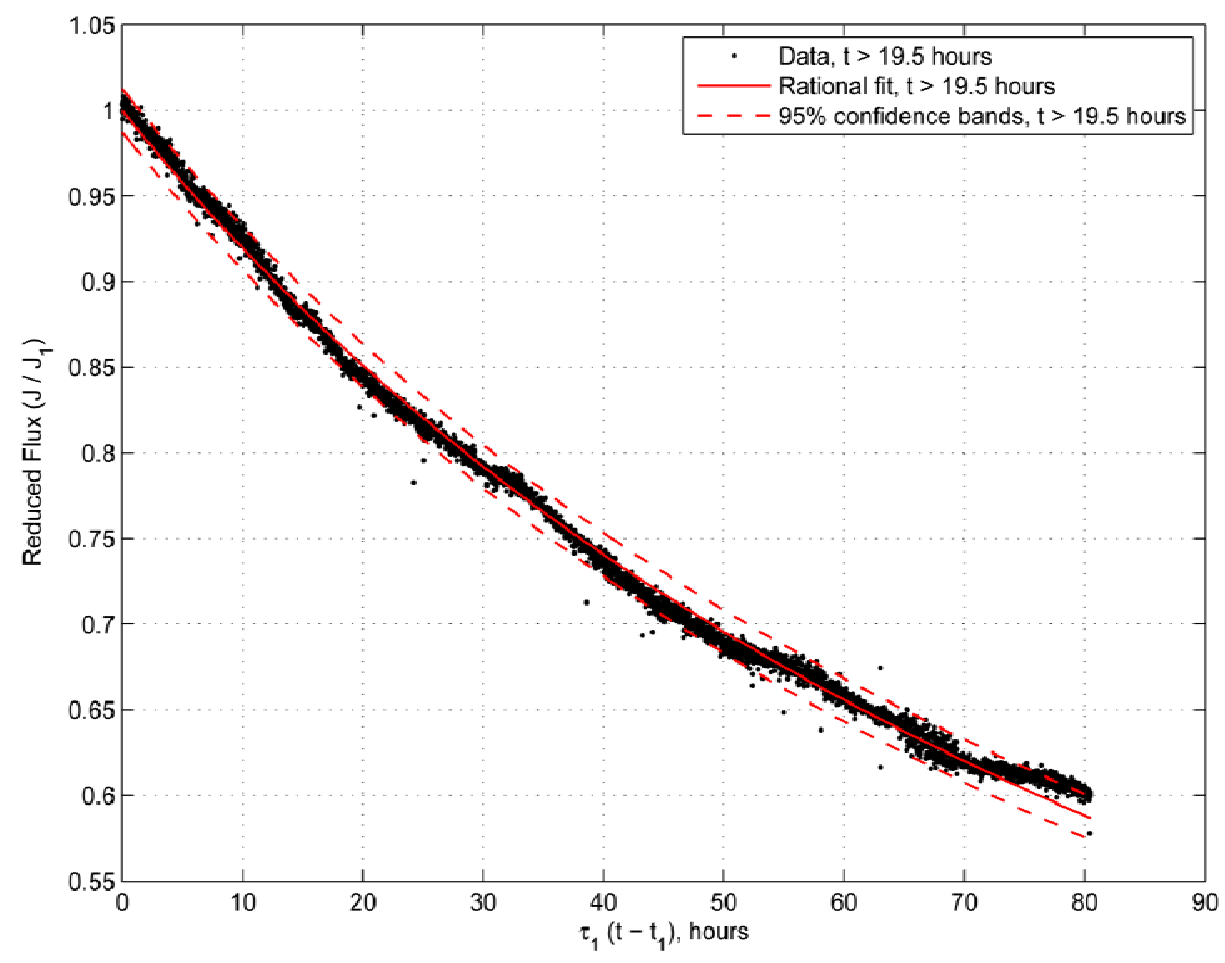

Figure A.37. Optimum Fit to S1T5 Flux-Time Data Using the Model of Equation 8.23. The bands of 95\% confidence for the fit are shown as dotted lines.

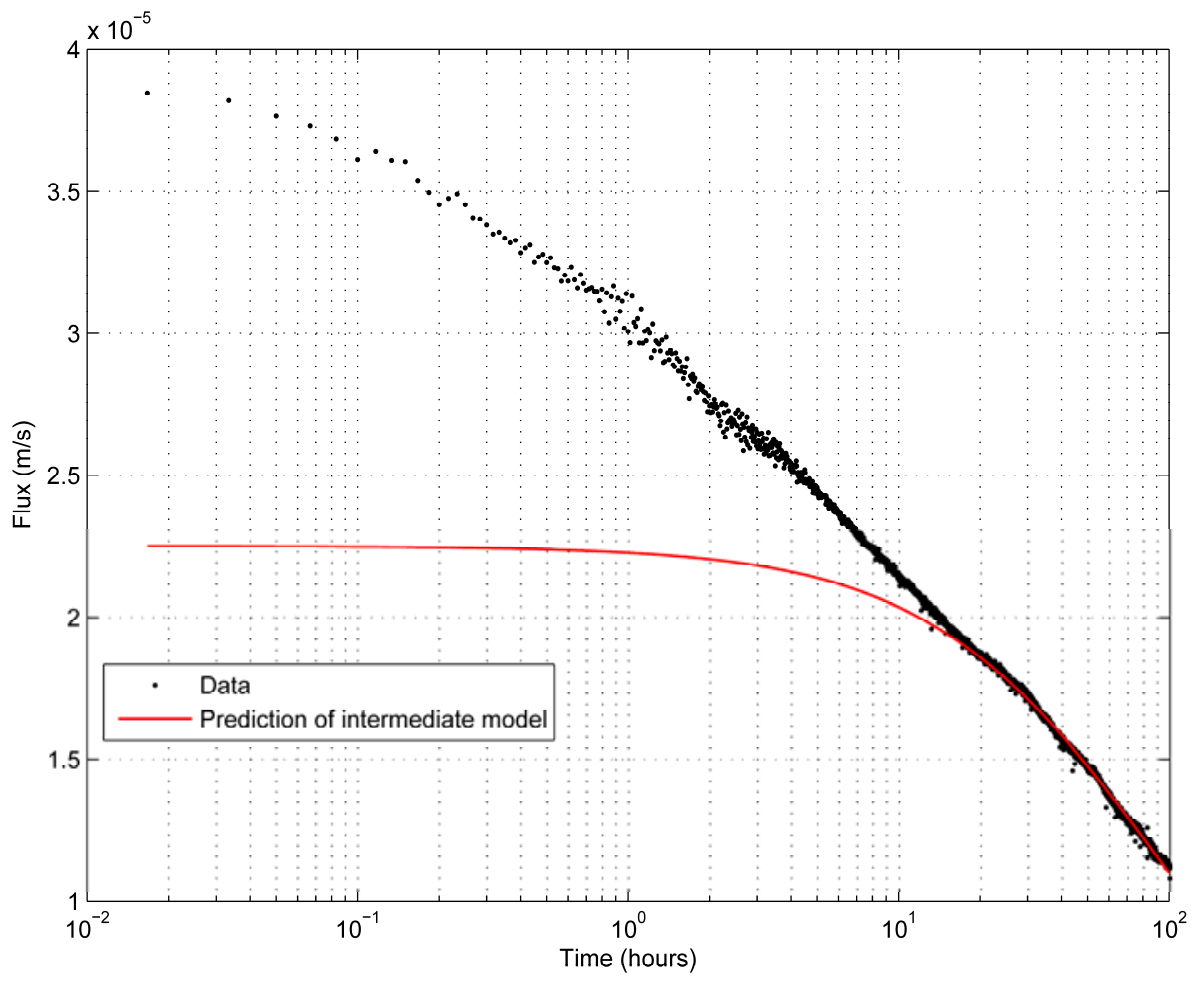

Figure A.38. Long Time Optimum Fit for S1T5 Data Using the Intermediate Mechanism Model 


\section{A.7 Mechanistic Modeling: Optimization for Cake Mechanism at Intermediate Times}

An example of an error plot from which the optimum was selected is given in Figure A.39. An example of the normal probability plot for the residuals of the optimum fit is given in Figure A.40. These figures correspond to the S1T1 figures described in Section 8.2 (see Figure 8.15 and Figure 8.16). What follows is the model fits for the other four tests (S1T2 - S1T5) in both shifted coordinates $\left(J / J_{2}, \tau_{2}\right)$ and the original coordinates $(J(\mathrm{~m} / \mathrm{s}), t(\mathrm{hr}))$. The error plot and normal probability plot are not supplied for these four tests but they are similar in nature to Figure A.39 and Figure A.40.

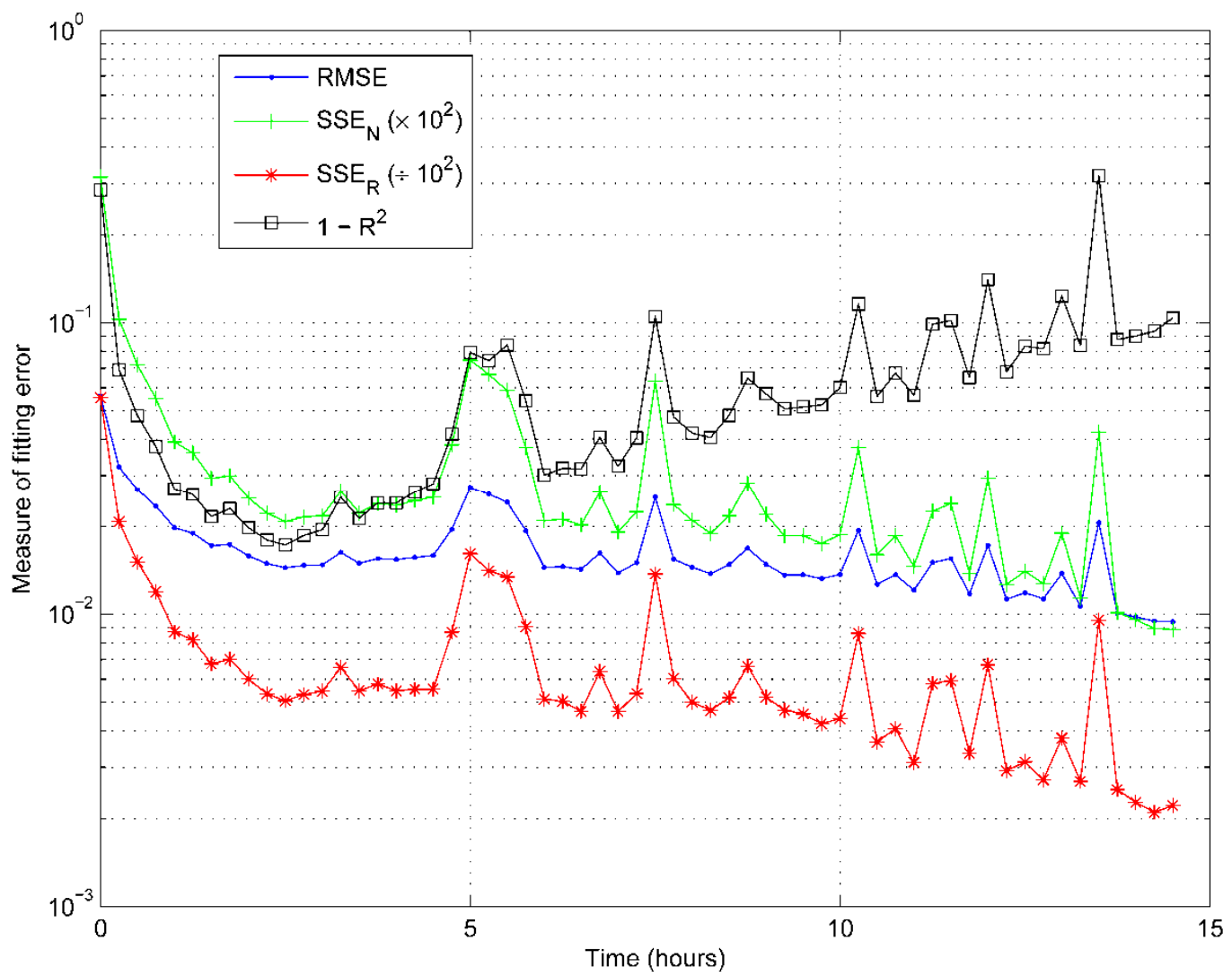

Figure A.39. Goodness-of-Fit Statistics for the Intermediate Time Cake Mechanism Model when Fit to the S1T1 Data. The statistics are plotted as a function of the time where the intermediate mechanism is assumed to first apply $\left(t_{2}\right): \mathrm{RMSE}=$ root-mean squared error, $\mathrm{SSE}_{\mathrm{N}}=$ sum of squares error normalized by number of data points, $\mathrm{SSE}_{\mathrm{R}}=$ sum of squares error normalized by data range, and $\mathrm{R}^{2}=$ coefficient of determination. 


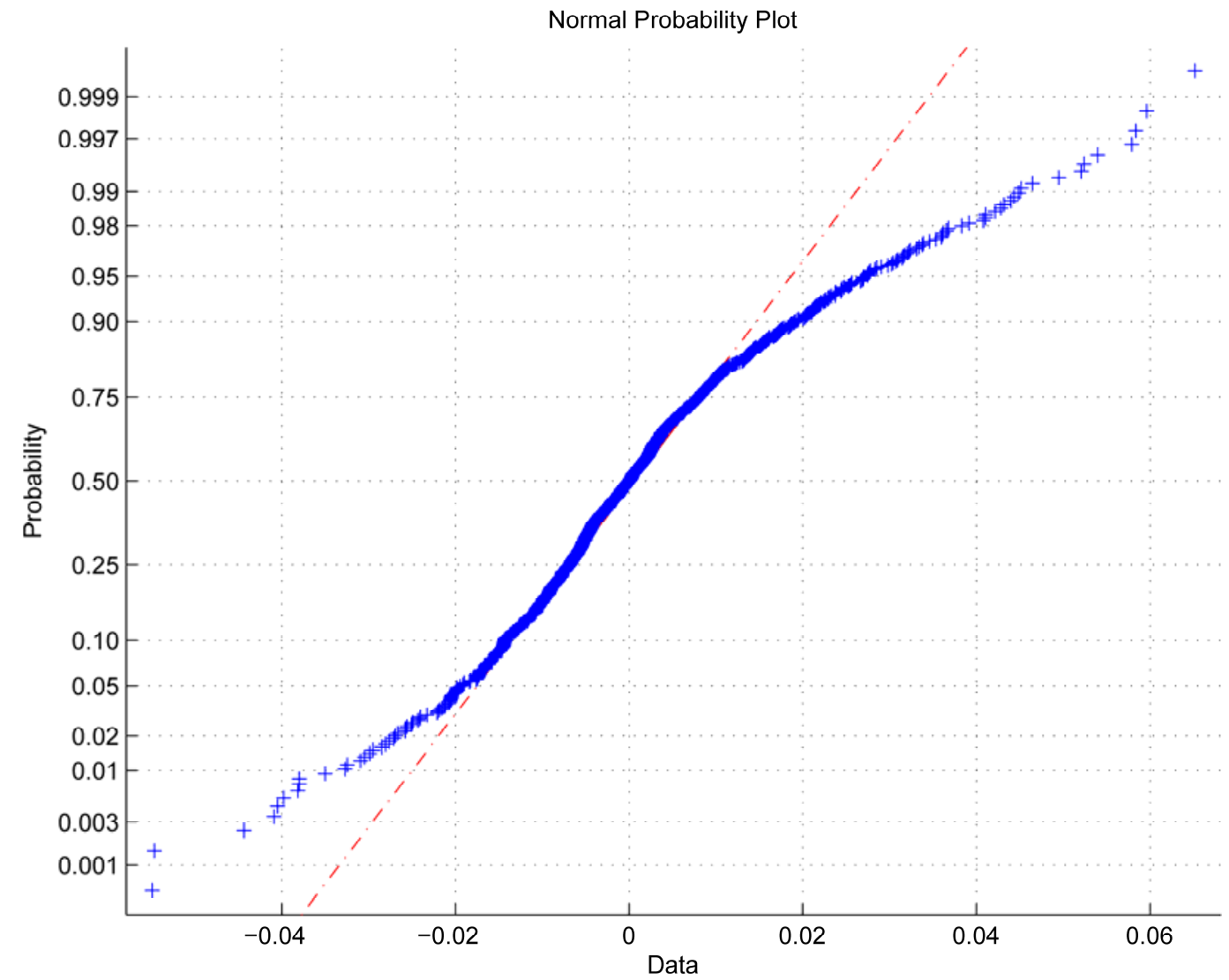

Figure A.40. Normal Probability Plot for the Residuals of the Optimum Fit to the S1T1 Data Using the Cake Mechanism. The residuals from the fit are given as blue '+', and the red dot-dash line is the expected behavior if the residuals are normally distributed with a mean of zero. 


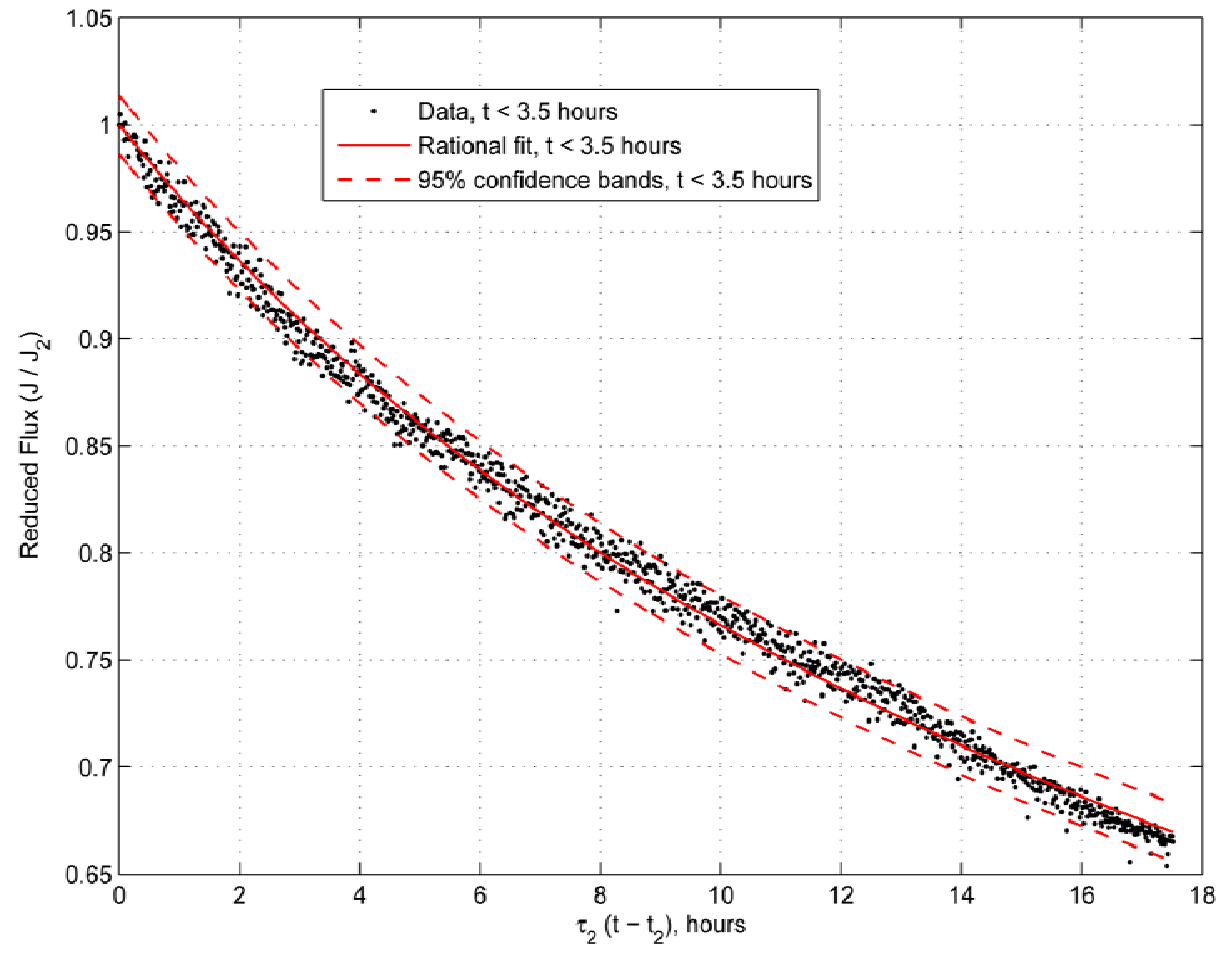

Figure A.41. Optimum Fit to S1T2 Flux-Time Data Using the Model of Equation 8.25. The bands of 95\% confidence for the fit are shown as dotted lines.

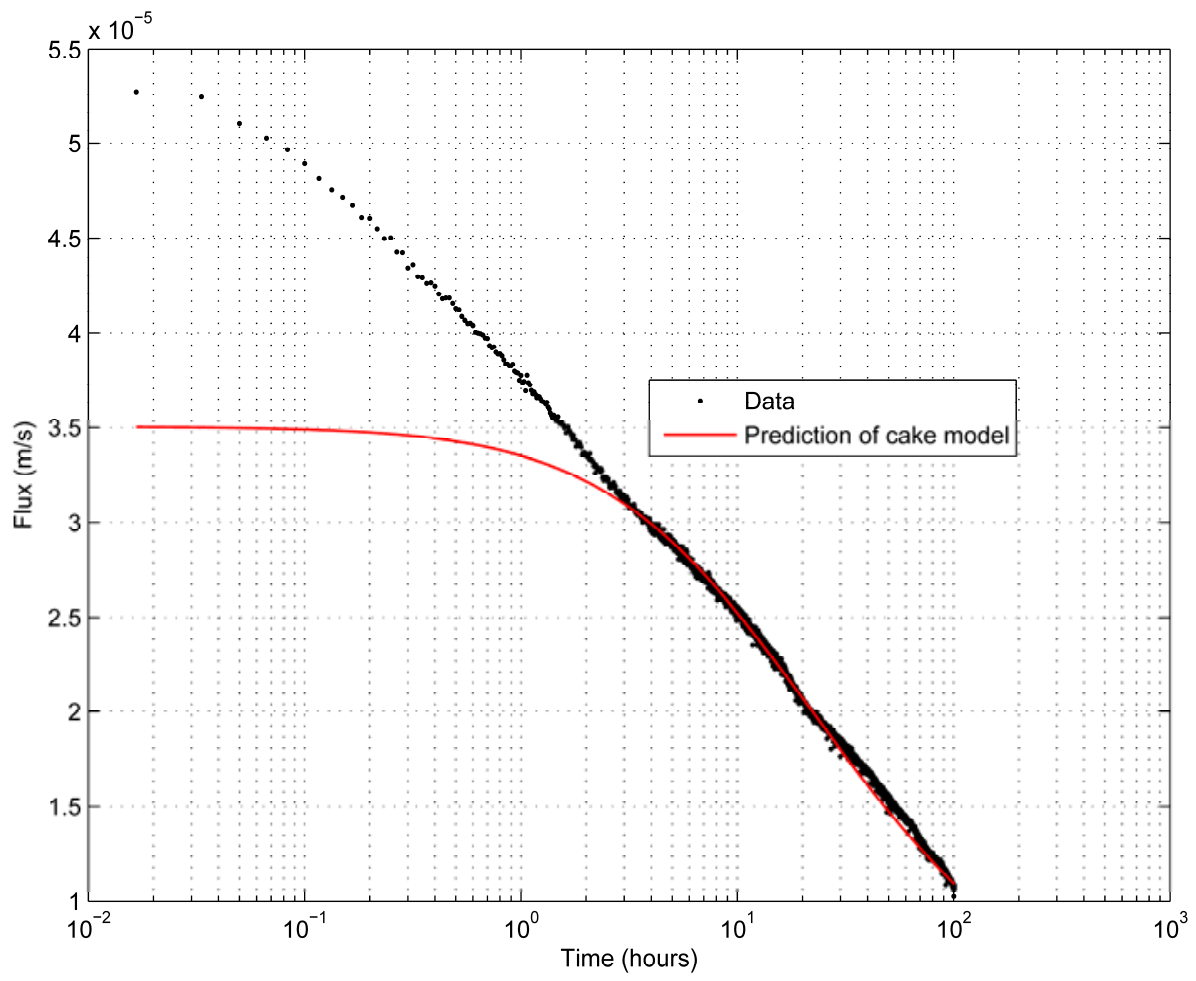

Figure A.42. Intermediate Time Optimum Fit for S1T2 Data Using the Cake Mechanism Model 


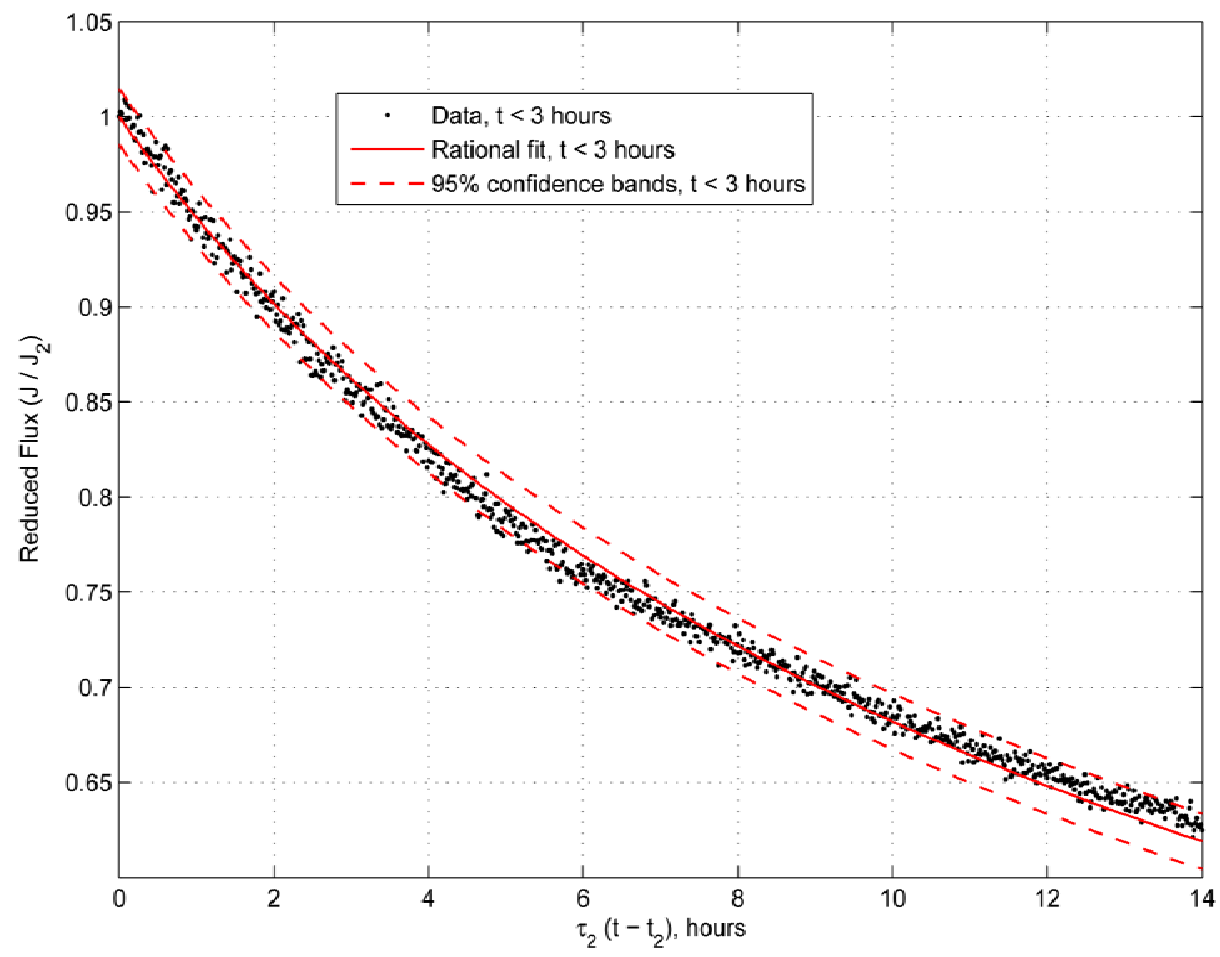

Figure A.43. Optimum Fit to S1T3 Flux-Time Data Using the Model of Equation 8.25. The bands of 95\% confidence for the fit are shown as dotted lines.

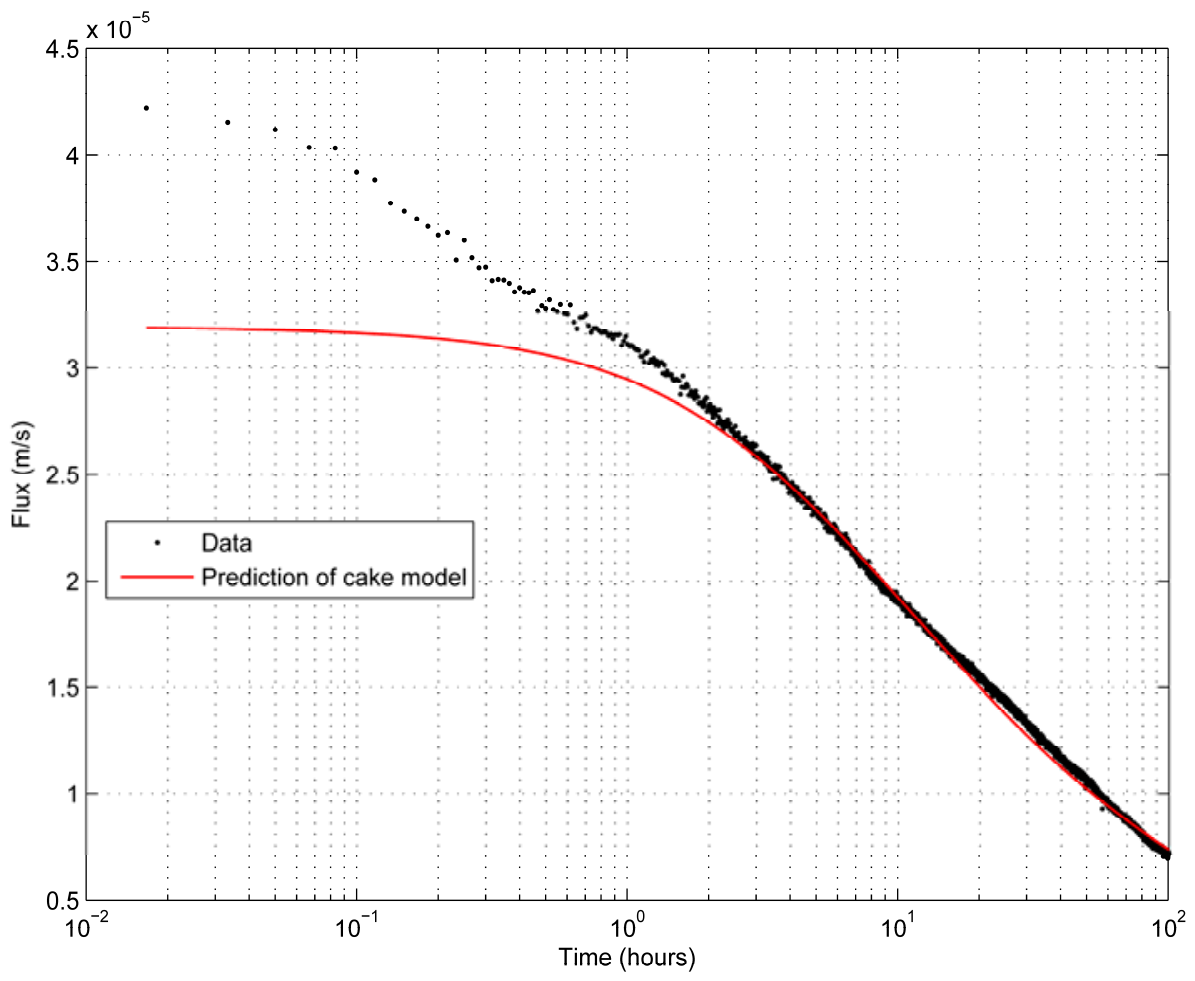

Figure A.44. Intermediate Time Optimum Fit for S1T3 Data Using the Cake Mechanism Model 


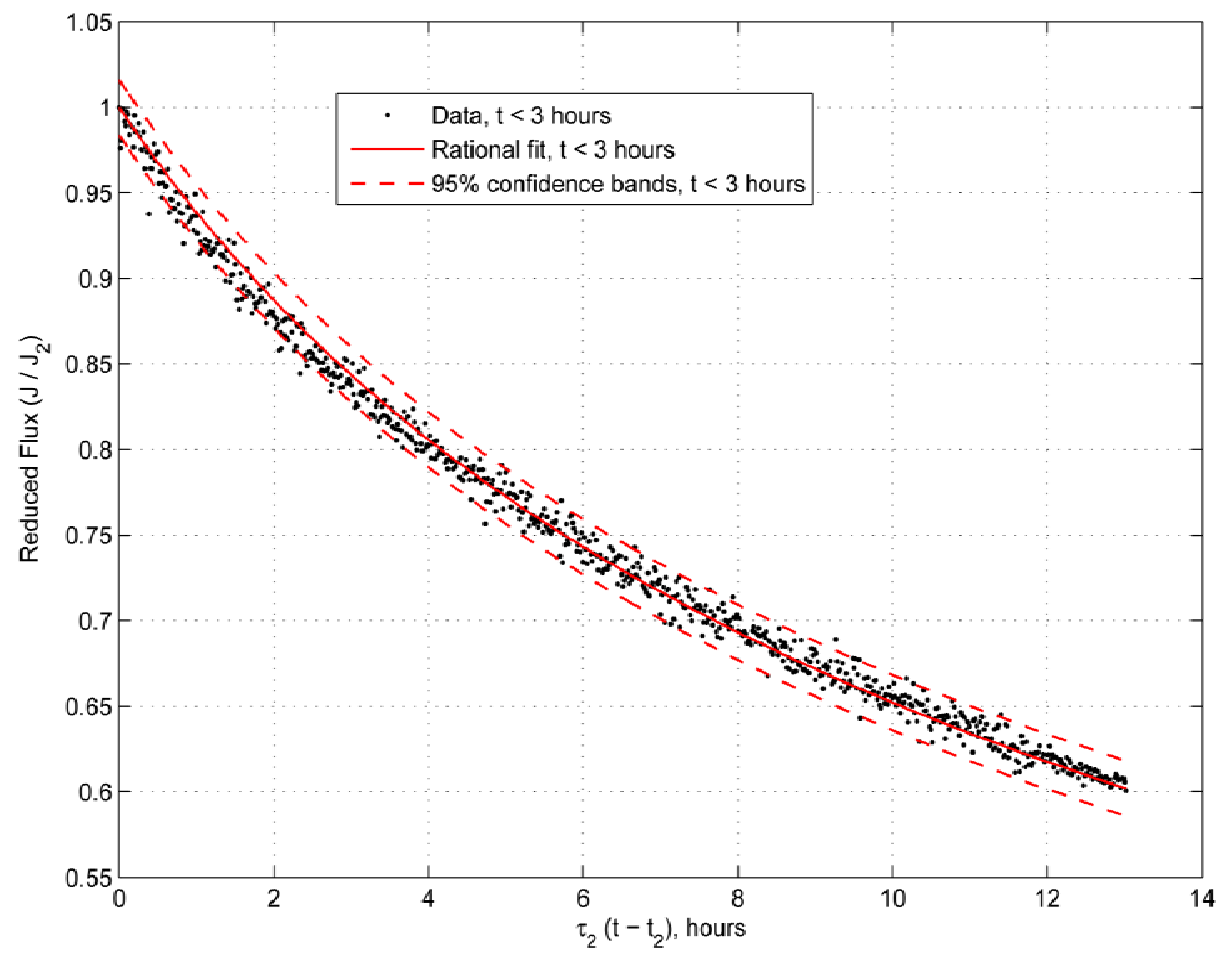

Figure A.45. Optimum Fit to S1T4 Flux-Time Data Using the Model of Equation 8.25. The bands of 95\% confidence for the fit are shown as dotted lines.

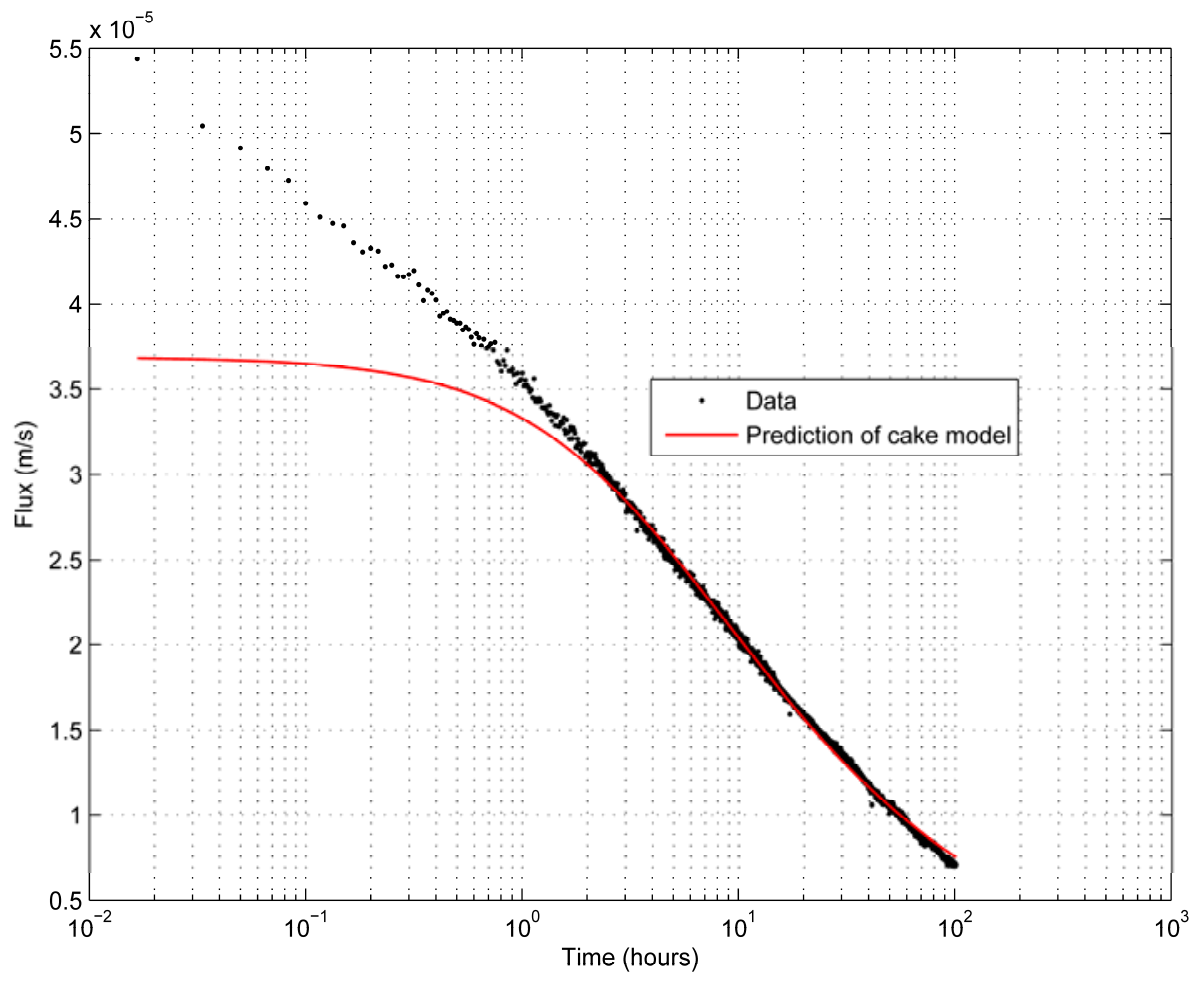

Figure A.46. Intermediate Time Optimum Fit for S1T4 Data Using the Cake Mechanism Model 


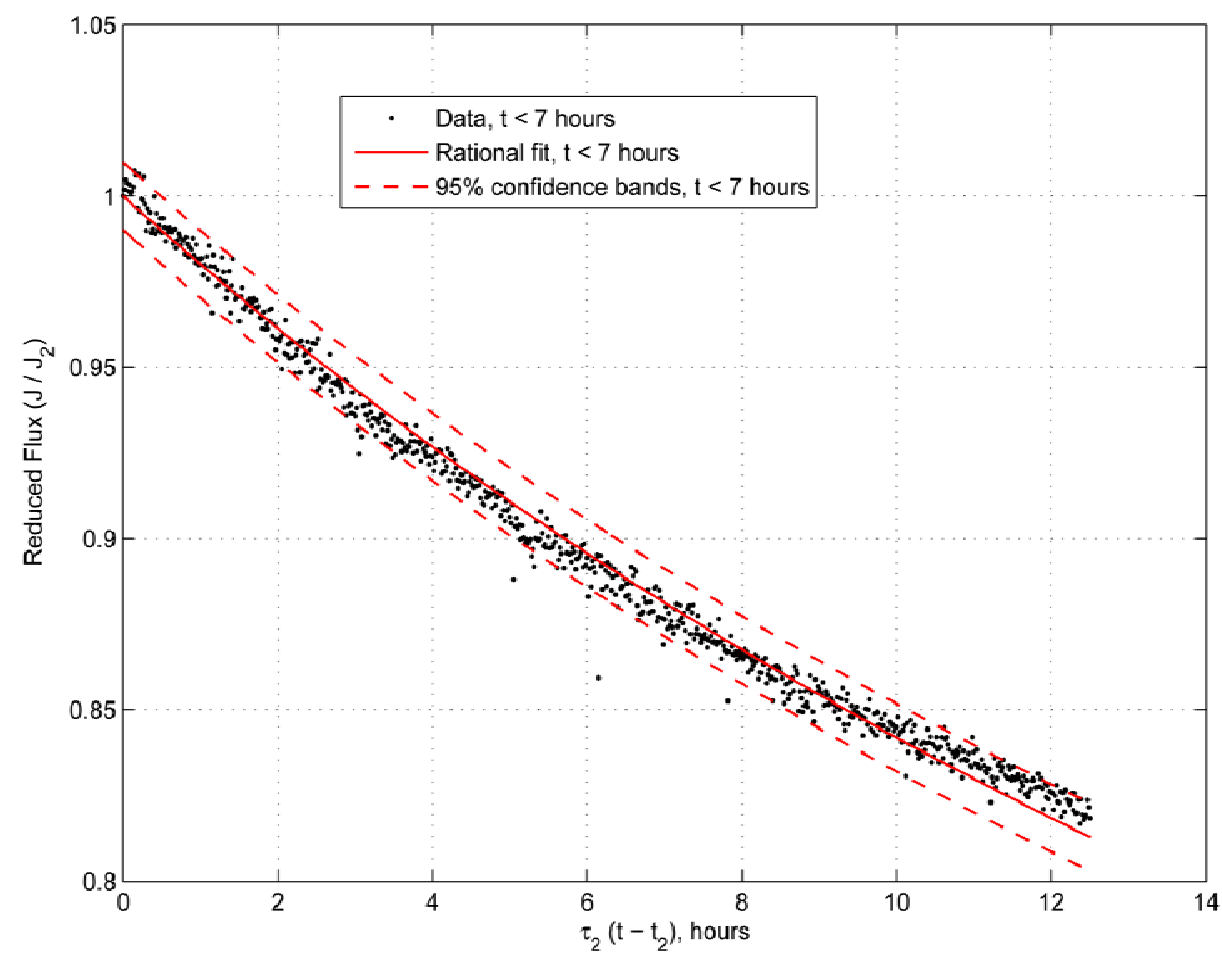

Figure A.47. Optimum Fit to S1T5 Flux-Time Data Using the Model of Equation 8.25. The bands of $95 \%$ confidence for the fit are shown as dotted lines.

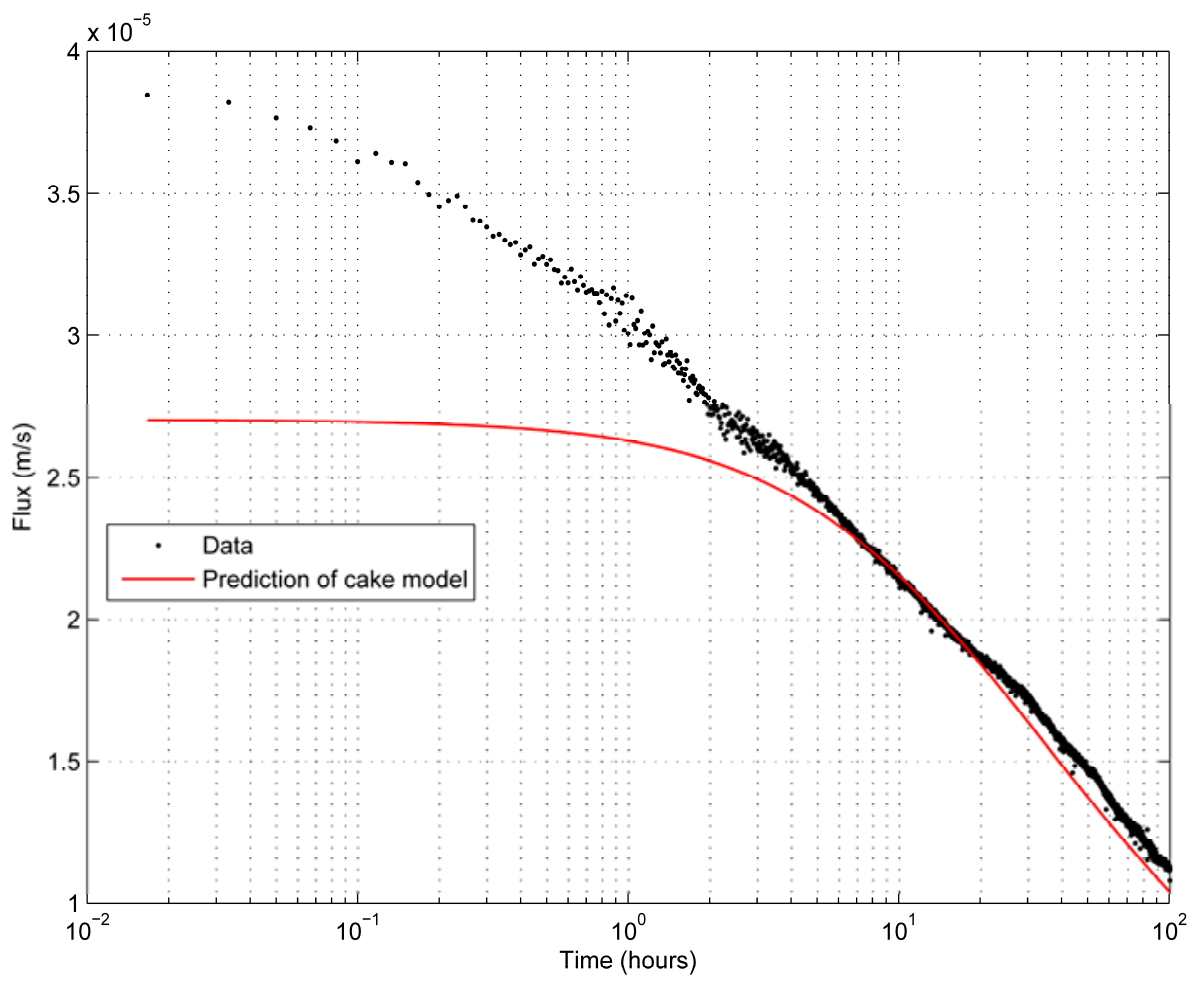

Figure A.48. Intermediate Time Optimum Fit for S1T5 Data Using the Cake Mechanism Model 

PNNL-20299

EMSP-RPT-008

\section{Distribution $^{(a)}$}

No. of

Copies

DOE Office of Environmental Management

H Johnson

N Machara

S Schneider

DOE Office of River Protection

T Fletcher

B Mauss

S Pfaff

$\underline{\text { Savannah River National Laboratory }}$

$M$ Duignan

D Herman

C Nash

F Pennebaker

B Wilmarth
No. of

Copies

Pacific Northwest National Laboratory

J Billing

C Burns

R Daniel

R Peterson

R. Russell

P Schonewill

R Shimskey

(a) All distribution will be made electronically. 


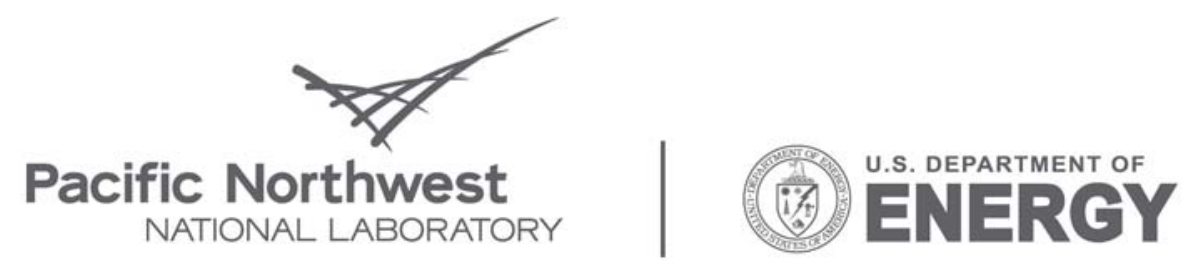

Proudly Operated by Battelle Since 1965

902 Battelle Boulevard

P.O. Box 999

Richland, WA 99352

1-888-375-PNNL (7665)

www.pnl.gov 